\title{
Pyridazine $N$-oxides as precursors to 2-aminofurans: Scope and limitations in complexity building reactions
}

\author{
Maribel Borger and James H. Frederich*
}

\section{Supporting Information}

Table of Contents

1. Materials and Methods

2. Experimental Procedures and Characterization Data

3. Supplementary Optimization Tables

4. Half-life Stability Experiments for $\mathbf{5 a}$ and $\mathbf{5 b}$

5. Additional Mechanism Experiments $(5 \mathbf{b} \rightarrow \mathbf{6})$

6. Detailed Experimental Setup

7. Photochemistry UV-Vis spectrum of $\mathbf{2 a}$

8. References

9. Copies of ${ }^{1} \mathrm{H}$ and ${ }^{13} \mathrm{C}$ NMR Spectra
$\mathrm{S} 1-\mathrm{S} 2$

S2-S19

$\mathrm{S} 19-\mathrm{S} 21$

S21-S22

S23-S24

S24-S25

S26

S26-S27

S28-S135

\section{Materials and Methods}

Unless otherwise stated, reactions were carried out in oven or flame-dried glassware under an atmosphere of nitrogen $\left(\mathrm{N}_{2}\right)$ using anhydrous solvents. Tetrahydrofuran (THF), 1,4-dioxane (dioxane), dichloromethane $\left(\mathrm{CH}_{2} \mathrm{Cl}_{2}\right)$, benzene $(\mathrm{PhH})$, toluene $(\mathrm{PhMe})$, and triethylamine $\left(\mathrm{Et}_{3} \mathrm{~N}\right)$ were dried by passage through activated alumina using a solvent purification system. Aryl boronic acids and 2-(4,4,5,5tetramethyl-1,3,2-dioxaborolan-2-yl)aniline were purchased and used as received. All other (hetero)arylboronic acid pinacol esters were prepared from commercial precursors following literature protocols as described in Figure $\mathrm{S} 1 .^{1-4} \mathrm{PdCl}_{2} \mathrm{dppf}, \mathrm{PdCl}_{2} \mathrm{dppf} \cdot \mathrm{CH}_{2} \mathrm{Cl}_{2}, \quad[\operatorname{Ir}(\operatorname{cod}) \mathrm{Cl}]_{2}, \quad[\mathrm{Ir}(\mathrm{OMe})(\mathrm{cod}) \mathrm{Cl}]_{2}$ $\mathrm{Rh}_{2} \mathrm{esp}_{2}$, and 4,4-di-tert-butyl bipyridine (dtbpy) were purchased and used as received. Pinacolborane (HBPin), pyrrolidine, acrylonitrile, trans-1,2-dicyanoethylene, dimethyl acetylenedicarboxylate, and diethyl silane $\left(\mathrm{Et}_{2} \mathrm{SiH}_{2}\right)$ were distilled prior to use from commercial sources. Propylidenemalonitrile was prepared following a literature procedure. ${ }^{5}$ All other commercial reagents were used as received.

Typically one representative reaction and yield of the product is described in detail; isolated yields appearing in Tables 1-2 and Schemes 2-3 are reported as the average yield obtained from duplicate experiments. Photochemical reactions were carried out in a Rayonet RPR-100 chamber photoreactor equipped with $24 \mathrm{~W}$ UV-lamps wherein $\sim 90 \%$ of emission is $350 \mathrm{~nm}$. Reaction temperatures were controlled using a temperature modulator. Column chromatography was carried out on silica gel 60 (240400 mesh) purchased from VWR. Thin layer chromatography (TLC) was performed using pre-coated, glass-backed plates $\left(\mathrm{SiO}_{2}, 60\right.$ PF254, $\left.0.25 \mathrm{~mm}\right)$ and visualized by exposure to UV light $(254 \mathrm{~nm})$ or by anisaldehyde, ceric ammonium molybdate, and potassium permanganate staining.

${ }^{1} \mathrm{H}$ NMR spectra were recorded at $400 \mathrm{MHz}$ or $600 \mathrm{MHz}$ and are reported relative to deuterated solvent signals. Data for ${ }^{1} \mathrm{H}$ NMR spectra are reported as follows: chemical shift $(\delta \mathrm{ppm})$, multiplicity, coupling constant (reported in $\mathrm{Hz}$ ), and integration. Splitting patterns are abbreviated as follows: singlet (s), doublet (d), triplet (t), quartet (q), quintet (quint), multiplet (m), broad (br), apparent (app), and combinations thereof. ${ }^{13} \mathrm{C}$ NMR spectra were recorded at 100 or $150 \mathrm{MHz}$. Data for ${ }^{13} \mathrm{C}$ NMR spectra are reported in order of carbon multiplicity $\left(\mathrm{C}=\right.$ quaternary, $\mathrm{CH}=$ methine, $\mathrm{CH}_{2}=$ methylene, $\mathrm{CH}_{3}=$ methyl) and chemical shift. Carbon multiplicity was established by DEPT135 and/or HSQC experiments. Reported melting points of solids are uncorrected. IR spectra were recorded on an FT-IR spectrometer and are reported in terms of frequency $\left(\mathrm{cm}^{-1}\right)$. Mass spectra were collected on an LCT spectrometer utilizing either electrospray $(\mathrm{ESI})$ or direct analysis in real time (DART) ionization techniques. 
<smiles>COc1ccc(N)c(Cc2ccccc2)c1</smiles>

S1

[ref. 1]<smiles>Cc1ccc(N)c(Cc2ccccc2)c1</smiles>

S7 [ref. 1]<smiles>Nc1ccc(Cl)cc1Cc1ccccc1</smiles>

S2

[ref. 2]<smiles>Cc1cccc(Cc2ccccc2)c1N</smiles>

S8

[ref. 1]<smiles>N#Cc1ccc(N)c(Cc2ccccc2)c1</smiles>

S3

[ref. 1]<smiles>Nc1cc(Cl)ncc1-c1ccccc1</smiles>

S9

[ref. 2]<smiles>Nc1ccc(C(F)(F)F)cc1Cc1ccccc1</smiles>

S4

[ref. 1]<smiles>Oc1ccccc1Cc1ccccc1</smiles>

S10

[ref. 3]<smiles>Nc1cc(F)ccc1-c1ccccc1</smiles>

S5

[ref. 1]<smiles>CC(C)(C)c1ccc(Br)c(O)c1</smiles>

S11

[ref. 4]<smiles>Nc1cccc(F)c1-c1ccccc1</smiles>

S6

[ref. 1]<smiles>COc1cc(C)cc(Cc2ccccc2)c1O</smiles>

S12 [ref. 4]

Figure S1. Syntheses of known (hetero)arylboronic acid pinacol esters used in this study.

\section{Experimental Procedures and Characterization Data}<smiles>[O-][n+]1nc(Cl)ccc1Cl</smiles>

1.

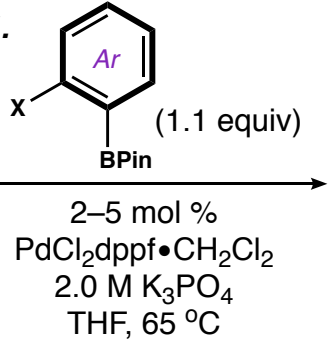

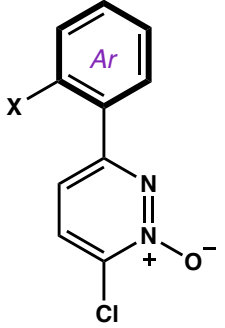

S13-S26
2.
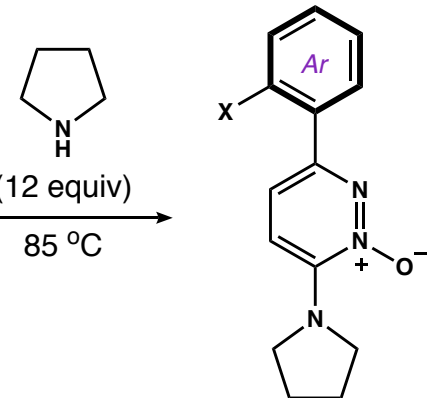

$2 \mathrm{~b}$ and $\mathbf{2 c}$ prepared as previously described in ref. 6<smiles>[O-][n+]1nc(-c2ccccc2)ccc1N1CCCC1</smiles>

$2 a$<smiles>[O-][n+]1nc(-c2ccc(F)cc2)ccc1N1CCCC1</smiles>

$2 a_{1}$<smiles>CN(C)c1ccc(-c2ccc(N3CCCC3)[n+]([O-])n2)cc1</smiles>

$2 \mathrm{a}_{2}$<smiles>[O-][n+]1nc(-c2ccc(Cl)cc2Cl)ccc1N1CCCC1</smiles>

$2 \mathbf{a}_{3}$<smiles>[O-][n+]1nc(-c2ccc3c(c2)OCO3)ccc1N1CCCC1</smiles>

$2 \mathrm{a}_{4}$<smiles>[O-][n+]1nc(-c2ccncc2)ccc1N1CCCC1</smiles>

$2 \mathrm{a}_{5}$

Figure S2. Two step assembly of photosubstrates 2.

General Procedure A: Preparation of 3-aryl-6-chloropyridazine- $\mathbf{N - o x i d e s ~ ( F i g u r e ~} \mathbf{1}$, step 1). A Schlenk tube was charged with 3,6-dichloropyridazine- $N$-oxide ${ }^{6}$ (1.0 equiv), boronic acid pinacol ester (1.1 equiv), and $\mathrm{PdCl}_{2} \mathrm{dppf} \cdot \mathrm{CH}_{2} \mathrm{Cl}_{2}\left(2-5 \mathrm{~mol} \%\right.$ ). The solids were placed under an atmosphere of $\mathrm{N}_{2}$ by three successive cycles of evacuation and backfilling. THF ( $3 \mathrm{~mL} / \mathrm{mmol}$ ) was added via syringe followed by $2.0 \mathrm{M}$ aq. $\mathrm{K}_{3} \mathrm{PO}_{4}$ (3 equiv). The resulting biphasic solution was rapidly stirred at $\mathrm{rt}$ for $\sim 1$ min before being warmed to $65{ }^{\circ} \mathrm{C}$. The reaction was monitored by TLC to follow loss of $3,6-$ dichloropyridazine- $\mathrm{N}$-oxide. Once this starting material was consumed (ca. 1-8 h), the reaction was cooled to rt. The resulting slurry was suspended in $\mathrm{CH}_{2} \mathrm{Cl}_{2}(25 \mathrm{~mL} / \mathrm{mmol})$ and washed with $\mathrm{H}_{2} \mathrm{O}(3 \times$ 
$25 \mathrm{~mL} / \mathrm{mmol})$ followed by saturated aq. $\mathrm{NH}_{4} \mathrm{Cl}(3 \times 25 \mathrm{~mL} / \mathrm{mmol})$. The organic layer was collected, dried over $\mathrm{Na}_{2} \mathrm{SO}_{4}$, filtered, and concentrated under reduced pressure. The resulting residue crude residue was purified by flash chromatography. ${ }^{7}$

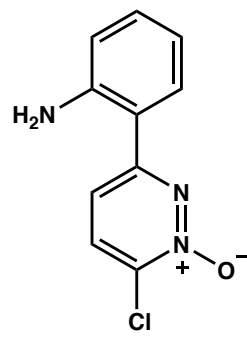

S13: $\mathrm{C}_{10} \mathrm{H}_{8} \mathrm{ClN}_{3} \mathrm{O}$ MW: 221.64

3-(2-aminophenyl)-6-chloropyridazine N-oxide (S13). Following General Procedure A, the title compound was prepared from 3,6-dichloropyridazine- $N$-oxide $(3.30 \mathrm{~g}, 20.0$ $\mathrm{mmol})$, 2-(4,4,5,5-tetramethyl-1,3,2-dioxaborolan-2-yl)aniline $(4.82 \mathrm{~g}, 22.0 \mathrm{mmol})$ and $\mathrm{PdCl}_{2} \mathrm{dppf} \cdot \mathrm{CH}_{2} \mathrm{Cl}_{2}(326 \mathrm{mg}, 0.40 \mathrm{mmol}, 2 \mathrm{~mol} \%$ ). After $45 \mathrm{~min}$, the reaction mixture was concentrated under reduced pressure. The mixture was digested in $\mathrm{CH}_{2} \mathrm{Cl}_{2}$ (45 $\mathrm{mL} / \mathrm{mmol}$ ) and passed through a pad of $\mathrm{SiO}_{2}(15 \mathrm{~g})$. The resulting crude residue was washed with acetone $(20 \mathrm{~mL})$ and provided $\mathbf{S} 13(3.55 \mathrm{~g}, 16.0 \mathrm{mmol}, 80 \%$ yield) as a yellow solid: $\mathbf{m p}=197-198{ }^{\circ} \mathrm{C} ;{ }^{1} \mathrm{H}$ NMR $\left(400 \mathrm{MHz}, \mathrm{CDCl}_{3}\right) \delta 7.81(\mathrm{~d}, \mathrm{~J}=8.6 \mathrm{~Hz}, 1 \mathrm{H})$, $7.47(\mathrm{~d}, J=7.8 \mathrm{~Hz}, 1 \mathrm{H}), 7.38(\mathrm{~d}, J=8.7 \mathrm{~Hz}, 1 \mathrm{H}), 7.27-7.24(\mathrm{~m}, 1 \mathrm{H}), 6.81-6.75(\mathrm{~m}$, $2 \mathrm{H}), 5.53(\mathrm{br} \mathrm{s}, 2 \mathrm{H}) ;{ }^{13} \mathrm{C}$ NMR $(150 \mathrm{MHz}$, DMSO-d 6 ) $\delta \mathrm{C:}$ 159.6, 147.8, 134.2, 115.7; CH: 135.8, 132.0, 130.1, 117.5, 117.3, 116.6; IR (thin-film) 3447, 3305, $757 \mathrm{~cm}^{-1}$; HRMS-ESI (m/z) $[\mathrm{M}+\mathrm{Na}]^{+}$ calculated for $\mathrm{C}_{10} \mathrm{H}_{8} \mathrm{CIN}_{3} \mathrm{ONa}=244.0254$; found 244.0249.

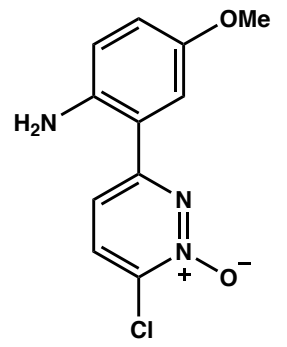

S14: $\mathrm{C}_{11} \mathrm{H}_{10} \mathrm{CIN}_{3} \mathrm{O}_{2}$ MW: 251.67

3-(2-amino-5-methoxyphenyl)-6-chloropyridazine-N-oxide (S14). Following General Procedure $A$, the title compound was prepared from 3,6-dichloropyridazine- $N$-oxide (97 mg, $0.59 \mathrm{mmol}$ ), S1 (162 mg, $0.65 \mathrm{mmol}$ ), and $\mathrm{PdCl}_{2} \mathrm{dppf} \cdot \mathrm{CH}_{2} \mathrm{Cl}_{2}$ (20 mg, 0.020 $\mathrm{mmol}, 3 \mathrm{~mol} \%)$. After $17 \mathrm{~h}$, purification by trituration with hexanes provided $\mathbf{S 1 4}$ (101 mg, $0.40 \mathrm{mmol}, 68 \%$ yield) as a yellow solid: $\mathbf{m p}=179-181{ }^{\circ} \mathrm{C} ;{ }^{1} \mathbf{H}$ NMR (400 $\left.\mathrm{MHz} \mathrm{CDCl}_{3}\right) \delta 7.82(\mathrm{~d}, J=8.6 \mathrm{~Hz}, 1 \mathrm{H}), 7.36(\mathrm{~d}, J=8.7 \mathrm{~Hz}, 1 \mathrm{H}), 6.98-6.97(\mathrm{~m}, 1 \mathrm{H})$, 6.92 (dd, $J=6.1,2.7 \mathrm{~Hz}, 1 \mathrm{H}), 6.74(\mathrm{~d}, J=8.8 \mathrm{~Hz}, 1 \mathrm{H}), 5.09$ (br s, 2H), 3.79 (s, 3H); ${ }^{13} \mathrm{C}$ NMR (150 MHz, DMSO-d 6$) \delta$ C: 159.3, 151.1, 142.0, 134.3, 116.2; CH: 135.8, 119.9, 118.9, 117.9, 113.6; $\mathbf{C H}_{3}: 56.0$; IR (thin-film) 3410, 2941, $1590 \mathrm{~cm}^{-1}$; HRMSESI $(\mathrm{m} / \mathrm{z})[\mathrm{M}+\mathrm{H}]^{+}$calculated for $\mathrm{C}_{11} \mathrm{H}_{11} \mathrm{CIN}_{3} \mathrm{O}_{2}=252.0534$; found 252.0537 .

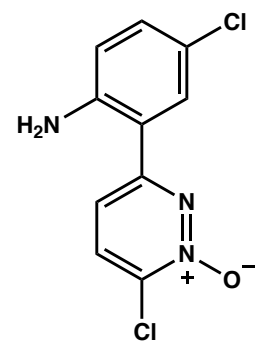

S15: $\mathrm{C}_{10} \mathrm{H}_{7} \mathrm{Cl}_{2} \mathrm{~N}_{3} \mathrm{O}$ $M W: 256.09$

3-(2-amino-5-chlorophenyl)-6-chloropyridazine-N-oxide (S15). Following General Procedure $A$, the title compound was prepared from 3,6-dichloropyridazine- $N$-oxide (750 mg, $4.55 \mathrm{mmol})$, S2 (1.27 g, $5.00 \mathrm{mmol}$ ), and $\mathrm{PdCl}_{2} \mathrm{dppf} \cdot \mathrm{CH}_{2} \mathrm{Cl}_{2}$ (115 mg, 0.14 mmol, $3 \mathrm{~mol} \%)$. After $3 \mathrm{~h}$, purification by flash chromatography $\left(\mathrm{SiO}_{2}, 3: 1\right.$ hexanes/acetone) provided $\mathbf{S 1 5}(822 \mathrm{mg}, 3.22 \mathrm{mmol}, 71 \%$ yield) as a yellow solid:

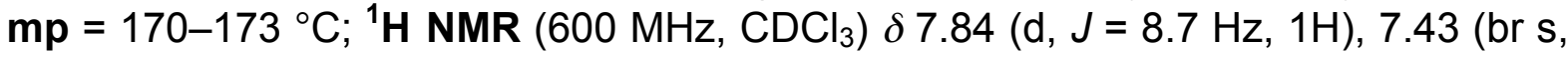
$1 \mathrm{H}), 7.35(\mathrm{~d}, J=8.8 \mathrm{~Hz}, 1 \mathrm{H}), 7.18(\mathrm{~d}, J=8.5 \mathrm{~Hz}, 1 \mathrm{H}), 6.71(\mathrm{~d}, J=8.7 \mathrm{~Hz}, 1 \mathrm{H}), 5.58$ (br s, $2 \mathrm{H}) ;{ }^{13} \mathrm{C}$ NMR $\left(150 \mathrm{MHz}, \mathrm{CDCl}_{3}\right) \delta \mathrm{C}: 158.5,145.4,135.1,122.3,116.4 ; \mathbf{C H}$ : 134.6, 132.0, 128.5, 119.2, 115.7; IR (thin-film) 3425, 1306, $736 \mathrm{~cm}^{-1}$; HRMS-ESI $(\mathrm{m} / \mathrm{z})[\mathrm{M}+\mathrm{Na}]^{+}$calculated for $\mathrm{C}_{10} \mathrm{H}_{7} \mathrm{Cl}_{2} \mathrm{~N}_{3} \mathrm{ONa}=277.9858$; found 277.9864 .

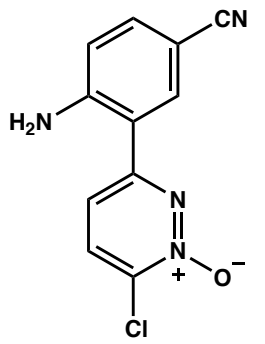

S16: $\mathrm{C}_{11} \mathrm{H}_{7} \mathrm{CIN}_{4} \mathrm{O}$ MW: 246.65

3-(2-amino-5-cyanophenyl)-6-chloropyridazine-N-oxide (S16). Following General Procedure $A$, the title compound was prepared from 3,6-dichloropyridazine- $N$-oxide (150 mg, $0.91 \mathrm{mmol}), \mathbf{S} 3$ (244 $\mathrm{mg}, 1.00 \mathrm{mmol})$ and $\mathrm{PdCl}_{2} \mathrm{dppf} \bullet \mathrm{CH}_{2} \mathrm{Cl}_{2}$ (39 $\mathrm{mg}, 0.048$ mmol, $5 \mathrm{~mol} \%)$. After $13 \mathrm{~h}$, purification by flash chromatography $\left(\mathrm{SiO}_{2}, 1: 1\right.$ hexanes/EtOAc) provided $\mathbf{S 1 6}(149 \mathrm{mg}, 0.61 \mathrm{mmol}, 67 \%$ yield) as a yellow solid: $\mathbf{m p}$ $=244-245{ }^{\circ} \mathrm{C} ;{ }^{1} \mathrm{H}$ NMR $\left(400 \mathrm{MHz}, \mathrm{DMSO}^{-\mathrm{d}_{6}}\right) \delta 8.36(\mathrm{br} \mathrm{s}, 1 \mathrm{H}), 7.95(\mathrm{br} \mathrm{s}, 1 \mathrm{H}), 7.73$ (br s, 1H), 7.54 (br s, 1H), 7.01 (br s, 2H), 6.88 (br s, 1H); ${ }^{13} \mathbf{C ~ N M R ~ ( 1 5 0 ~ M H z , ~}$ DMSO-d $\left._{6}\right) \delta$ C: 157.7, 151.3, 120.3, 116.1, 97.2, 40.5; CH: 136.3, 135.2, 134.7, 118.0, 117.3; IR (thin-film) 3558, 2213, $750 \mathrm{~cm}^{-1}$; HRMS-ESI $(\mathrm{m} / \mathrm{z})[\mathrm{M}+\mathrm{H}]^{+}$calculated for $\mathrm{C}_{11} \mathrm{H}_{8} \mathrm{CIN}_{4} \mathrm{O}=247.0381$; found 247.0376 . 


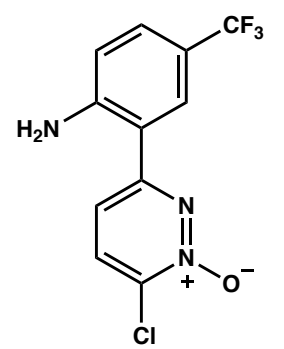

S17: $\mathrm{C}_{11} \mathrm{H}_{7} \mathrm{CIF}_{3} \mathrm{~N}_{3} \mathrm{O}$ MW: 289.64

3-(2-amino-5-(trifluoromethyl)phenyl)-6-chloropyridazine-N-oxide (S17). Following General Procedure A, the title compound was prepared from 3,6-dichloropyridazine$\mathrm{N}$-oxide (383 mg, $2.32 \mathrm{mmol}), \mathbf{S} 4(734 \mathrm{mg}, 2.56 \mathrm{mmol})$, and $\mathrm{PdCl}_{2} \mathrm{dppf} \bullet \mathrm{CH}_{2} \mathrm{Cl}_{2}(57$ $\mathrm{mg}, 0.070 \mathrm{mmol}, 3 \mathrm{~mol} \%)$. After $3 \mathrm{~h}$, purification by flash chromatography $\left(\mathrm{SiO}_{2}\right.$, $4: 1 \rightarrow 3: 1$ hexanes/acetone) provided $\mathbf{S} 17(621 \mathrm{mg}, 2.15 \mathrm{mmol}, 93 \%$ yield) as a yellow solid: $\mathbf{m p}=171-172{ }^{\circ} \mathrm{C} ;{ }^{1} \mathrm{H}$ NMR $\left(400 \mathrm{MHz}, \mathrm{CDCl}_{3}\right) \delta 7.87(\mathrm{~d}, \mathrm{~J}=8.7 \mathrm{~Hz}$, 1H), $7.74(\mathrm{br} \mathrm{s}, 1 \mathrm{H}), 7.46(\mathrm{dd}, J=8.5,1.6 \mathrm{~Hz}, 1 \mathrm{H}), 7.43(\mathrm{~d}, J=8.8 \mathrm{~Hz}, 1 \mathrm{H}), 6.81(\mathrm{~d}$, $J=8.6 \mathrm{~Hz}, 1 \mathrm{H}), 5.63(\mathrm{br} \mathrm{s}, 2 \mathrm{H}) ;{ }^{13} \mathbf{C}$ NMR $\left(150 \mathrm{MHz}, \mathrm{CDCl}_{3}\right) \delta \mathrm{C}: 158.6,149.4$, 135.2, 124.5 (q, $J=271 \mathrm{~Hz}), 119.4(\mathrm{q}, J=33 \mathrm{~Hz}), 114.2 ; \mathrm{CH}: 134.7,128.8,128.7$, 117.7, 115.6; IR (thin-film) 3433, 3191, $1015 \mathrm{~cm}^{-1}$; HRMS-ESI $(\mathrm{m} / \mathrm{z})[\mathrm{M}+\mathrm{H}]^{+}$calculated for $\mathrm{C}_{11} \mathrm{H}_{8} \mathrm{ClF}_{3} \mathrm{~N}_{3} \mathrm{O}=290.0303$; found 290.0314 .

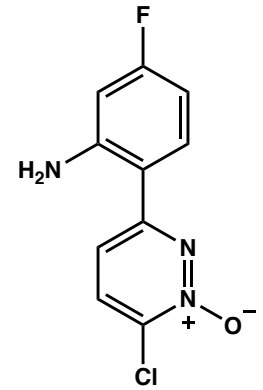

S18: $\mathrm{C}_{10} \mathrm{H}_{7} \mathrm{CIFN}_{3} \mathrm{O}$ MW: 239.63

\section{3-(2-amino-4-fluorophenyl)-6-chloropyridazine- $N$-oxide}

(S18).

Following

a modification of General Procedure A, the title compound was prepared from 3,6dichloropyridazine- $N$-oxide $(250 \mathrm{mg}, 1.52 \mathrm{mmol})$, S5 $(396 \mathrm{mg}, 1.67 \mathrm{mmol})$, and $\mathrm{PdCl}_{2} \mathrm{dppf} \cdot \mathrm{CH}_{2} \mathrm{Cl}_{2}(56 \mathrm{mg}, 0.076 \mathrm{mmol}, 5 \mathrm{~mol} \%$ ). After $3 \mathrm{~h}$, material was filtered and washed with cold THF ( $5 \mathrm{~mL})$. Purification by recrystallization with PhMe provided S18 (269 mg, $1.13 \mathrm{mmol}, 74 \%$ yield) as a gray solid: $\mathrm{mp}=193-194{ }^{\circ} \mathrm{C} ;{ }^{1} \mathbf{H}$ NMR $\left(600 \mathrm{MHz}, \mathrm{DMSO}-\mathrm{d}_{6}\right) \delta 8.28(\mathrm{~d}, J=8.2 \mathrm{~Hz}, 1 \mathrm{H}), 7.63(\mathrm{~d}, J=8.5 \mathrm{~Hz}, 1 \mathrm{H}), 7.58(\mathrm{t}, J=$ $7.2 \mathrm{~Hz}, 1 \mathrm{H}), 6.59(\mathrm{~d}, J=11.5 \mathrm{~Hz}, 1 \mathrm{H}), 6.48(\mathrm{br} \mathrm{s}, 3 \mathrm{H}) ;{ }^{13} \mathrm{C}$ NMR $(150 \mathrm{MHz}$, DMSO$\left.\mathrm{d}_{6}\right) \delta \mathrm{C}: 164.8(\mathrm{~d}, J=249), 158.8,150.1(\mathrm{~d}, J=13 \mathrm{~Hz}), 134.2,112.6$; $\mathrm{CH}: 135.9$, $132.7(\mathrm{~d}, J=11 \mathrm{~Hz}$ ), 103.8 (d, $J=23 \mathrm{~Hz}$ ), 102.4 (d, $J=25 \mathrm{~Hz}$ ); IR (thin-film) 3417, 3286, $1095 \mathrm{~cm}^{-1}$; HRMS-DART $(\mathrm{m} / \mathrm{z})[\mathrm{M}+\mathrm{H}]^{+}$calculated for $\mathrm{C}_{10} \mathrm{H}_{8} \mathrm{ClFN}_{3} \mathrm{O}=240.0334$; found 240.0344 .

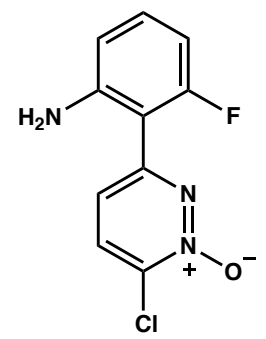

S19: $\mathrm{C}_{10} \mathrm{H}_{7} \mathrm{ClFN}_{3} \mathrm{O}$ MW: 239.63

3-(2-amino-6-fluorophenyl)-6-chloropyridazine 1-oxide (S19). Following General Procedure $\mathrm{A}$, the title compound was prepared from 3,6-dichloropyridazine- $\mathrm{N}$-oxide (75 mg, $0.46 \mathrm{mmol}), \mathbf{S} 6$ (119 mg, $0.50 \mathrm{mmol})$, and $\mathrm{PdCl}_{2} \mathrm{dppf} \cdot \mathrm{CH}_{2} \mathrm{Cl}_{2}(19 \mathrm{mg}, 0.023$ mmol, $5 \mathrm{~mol} \%)$. After $4 \mathrm{~h}$, purification by flash chromatography $\left(\mathrm{SiO}_{2}, 9: 1\right.$ hexanes/EtOAc) provided $\mathbf{S} 19$ (29 $\mathrm{mg}, 0.12 \mathrm{mmol}, 27 \%$ yield) as a yellow solid: $\mathbf{m p}$ $=154-156{ }^{\circ} \mathrm{C} ;{ }^{1} \mathrm{H}$ NMR $\left(400 \mathrm{MHz}, \mathrm{CDCl}_{3}\right) \delta 7.83(\mathrm{~d}, J=8.7 \mathrm{~Hz}, 1 \mathrm{H}), 7.35(\mathrm{dd}, J=$ 8.6, 3.6 Hz, 1H), 7.19-7.13 (m, 1H), $6.55(\mathrm{~d}, J=8.0 \mathrm{~Hz}, 1 \mathrm{H}), 6.51$ (dd, $J=8.7,2.8$ $\mathrm{Hz}, 1 \mathrm{H}), 5.19$ (br s, 2H); ${ }^{13} \mathrm{C}$ NMR $\left(150 \mathrm{MHz}, \mathrm{CDCl}_{3}\right) \delta \mathrm{C}: 161.6(\mathrm{~d}, J=247 \mathrm{~Hz})$, 154.9, 147.7 (d, $J=5 \mathrm{~Hz}), 135.6,106.4(\mathrm{~d}, J=15 \mathrm{~Hz}) ; \mathrm{CH}: 134.4,132.2$ (d, $J=12$ $\mathrm{Hz}$ ), 120.1 (d, $J=11 \mathrm{~Hz}$ ), 112.9 (d, $J=2 \mathrm{~Hz}$ ), 104.6 (d, $J=24 \mathrm{~Hz}$ ); IR (thin-film) 3456, 1323, $1088 \mathrm{~cm}^{-1}$; HRMS-ESI $(\mathrm{m} / \mathrm{z})[\mathrm{M}+\mathrm{H}]^{+}$calculated for $\mathrm{C}_{10} \mathrm{H}_{8} \mathrm{ClFN}_{3} \mathrm{O}=240.0334$; found 240.0328.

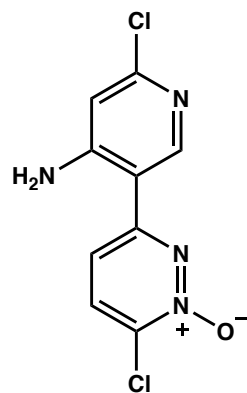

S20: $\mathrm{C}_{9} \mathrm{H}_{6} \mathrm{Cl}_{2} \mathrm{~N}_{4} \mathrm{O}$ MW: 257.07
3-(4-amino-6-chloropyridin-3-yl)-6-chloropyridazine-N-oxide (S20). Following a modification of General Procedure A, the title compound was prepared from 3,6dichloropyridazine- $N$-oxide (147 $\mathrm{mg}, 0.89 \mathrm{mmol}), \mathbf{S 9}(249 \mathrm{mg}, 0.98 \mathrm{mmol}$ ), and $\mathrm{PdCl}_{2} \mathrm{dppf} \cdot \mathrm{CH}_{2} \mathrm{Cl}_{2}(37 \mathrm{mg}, 0.045 \mathrm{mmol}, 5 \mathrm{~mol} \%)$. After $16 \mathrm{~h}$, purification by recrystallization with PhMe provided $\mathbf{S 2 0}(204 \mathrm{mg}, 0.80 \mathrm{mmol}, 89 \%$ yield) as a gray solid: $\mathrm{mp}=273-275{ }^{\circ} \mathrm{C}$ (decomp); ${ }^{1} \mathrm{H}$ NMR $\left(600 \mathrm{MHz}, \mathrm{DMSO}-\mathrm{d}_{6}\right) \delta 8.36$ (d, J = 8.6 $\mathrm{Hz}, 1 \mathrm{H}), 8.30(\mathrm{~s}, 1 \mathrm{H}), 7.69(\mathrm{~d}, \mathrm{~J}=8.6 \mathrm{~Hz}, 1 \mathrm{H}), 7.11$ (br s, 2H), $6.76(\mathrm{~s}, 1 \mathrm{H}) ;{ }^{13} \mathrm{C}$ NMR (150 MHz, DMSO-d 6 ) $\delta$ C: 156.5, 154.7, 152.1, 135.5, 113.0; CH: 150.9, 136.3, 117.6, 108.8; IR (thin-film) 3409, 1554, $1363 \mathrm{~cm}^{-1}$; HRMS-DART (m/z) [M+H] $]^{+}$ calculated for $\mathrm{C}_{9} \mathrm{H}_{7} \mathrm{Cl}_{2} \mathrm{~N}_{4} \mathrm{O}=256.9991$; found 256.9996 . 


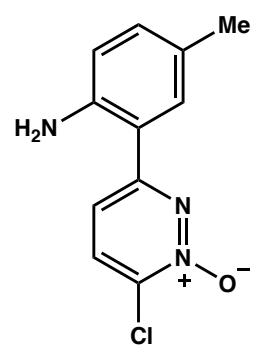

S21: $\mathrm{C}_{11} \mathrm{H}_{10} \mathrm{CIN}_{3} \mathrm{O}$ MW: 235.67

3-(2-amino-5-methylphenyl)-6-chloropyridazine-N-oxide (S21). Following General Procedure $\mathrm{A}$, the title compound was prepared from 3,6-dichloropyridazine- $\mathrm{N}$-oxide (247 mg, $1.52 \mathrm{mmol}), \mathbf{S 7}$ (386 mg, $1.65 \mathrm{mmol})$ and $\mathrm{PdCl}_{2} \mathrm{dppf} \cdot \mathrm{CH}_{2} \mathrm{Cl}_{2}(37 \mathrm{mg}, 0.045$ $\mathrm{mmol}, 3 \mathrm{~mol} \%)$. After $2 \mathrm{~h}$, purification by trituration with PhMe $(3 \mathrm{~mL})$ provided $\mathbf{S} 21$ (289 mg, $1.20 \mathrm{mmol}, 82 \%$ yield) as a yellow solid: $\mathrm{mp}=191-194{ }^{\circ} \mathrm{C} ;{ }^{1} \mathrm{H}$ NMR $(400$ $\left.\mathrm{MHz}, \mathrm{CDCl}_{3}\right) \delta 7.79(\mathrm{~d}, J=8.7 \mathrm{~Hz}, 1 \mathrm{H}), 7.38(\mathrm{~d}, J=8.7 \mathrm{~Hz}, 1 \mathrm{H}), 7.25(\mathrm{~s}, 1 \mathrm{H}), 7.07$ $(\mathrm{d}, J=7.8 \mathrm{~Hz}, 1 \mathrm{H}), 6.69(\mathrm{~d}, J=8.2 \mathrm{~Hz}, 1 \mathrm{H}), 5.32(\mathrm{~s}, 2 \mathrm{H}), 2.28(\mathrm{~s}, 3 \mathrm{H}) ;{ }^{13} \mathrm{C}$ NMR $(150$ $\left.\mathrm{MHz}, \mathrm{CDCl}_{3}\right) \delta \mathrm{C}: 159.8,144.4,134.4,127.1,115.7 ; \mathrm{CH}: 134.3,133.2,129.3,118.0$, 116.1; $\mathbf{C H}_{3}$ : 20.4; IR (thin-film) 3427, 2916, $1302 \mathrm{~cm}^{-1}$; HRMS-ESI $(\mathrm{m} / \mathrm{z})[\mathrm{M}+\mathrm{H}]^{+}$ calculated for $\mathrm{C}_{11} \mathrm{H}_{11} \mathrm{CIN}_{3} \mathrm{O}=236.0585$; found 236.0582.

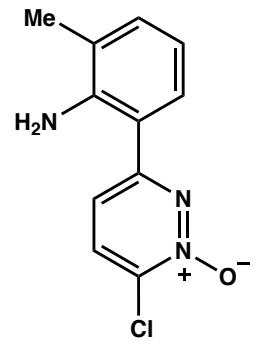

S22: $\mathrm{C}_{11} \mathrm{H}_{10} \mathrm{CIN}_{3} \mathrm{O}$ MW: 235.67

3-(2-amino-3-methylphenyl)-6-chloropyridazine-N-oxide (S22). Following General Procedure $\mathrm{A}$, the title compound was prepared from 3,6-dichloropyridazine- $\mathrm{N}$-oxide (184 mg, $1.12 \mathrm{mmol}), \mathbf{S} 8(288 \mathrm{mg}, 1.24 \mathrm{mmol})$ and $\mathrm{PdCl}_{2} \mathrm{dppf} \cdot \mathrm{CH}_{2} \mathrm{Cl}_{2}(42 \mathrm{mg}, 0.056$ mmol, $5 \mathrm{~mol} \%)$. After $1 \mathrm{~h}$, purification by flash chromatography $\left(\mathrm{SiO}_{2}, 1: 1\right.$ hexanes/EtOAc) provided $\mathbf{S} 22$ (213 $\mathrm{mg}, 0.91 \mathrm{mmol}, 81 \%$ yield) as a yellow solid: $\mathbf{m p}$ $=158-160{ }^{\circ} \mathrm{C} ;{ }^{1} \mathrm{H}$ NMR $\left(400 \mathrm{MHz}, \mathrm{CDCl}_{3}\right) \delta 7.80(\mathrm{~d}, J=8.7 \mathrm{~Hz}, 1 \mathrm{H}), 7.36(\mathrm{t}, J=9.4$ $\mathrm{Hz}, 1 \mathrm{H}), 7.18(\mathrm{~d}, J=7.3 \mathrm{~Hz} 1 \mathrm{H}), 6.73(\mathrm{t}, J=7.7 \mathrm{~Hz}, 1 \mathrm{H}), 5.44(\mathrm{br} \mathrm{s}, 2 \mathrm{H}), 2.22(\mathrm{~s}, 3 \mathrm{H})$; ${ }^{13} \mathrm{C}$ NMR $\left(150 \mathrm{MHz}, \mathrm{CDCl}_{3}\right) \delta$ C: $160.1,145.0,124.0,115.2$; CH: $134.4,133.2$, 127.5, 117.2, 116.5; $\mathbf{C H}_{3}$ : 17.9; IR (thin-film) 3428, 2975, $1322 \mathrm{~cm}^{-1}$; HRMS-ESI $(\mathrm{m} / \mathrm{z})[\mathrm{M}+\mathrm{H}]^{+}$calculated for $\mathrm{C}_{11} \mathrm{H}_{11} \mathrm{CIN}_{3} \mathrm{O}=236.0585$; found 236.0586 .

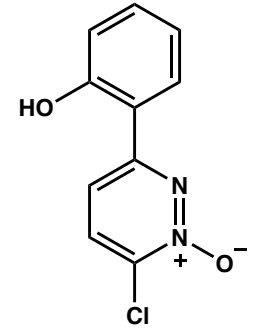

S23: $\mathrm{C}_{10} \mathrm{H}_{7} \mathrm{CIN}_{2} \mathrm{O}_{2}$ MW: 222.63

6-chloro-3-(2-hydroxyphenyl)pyridazine-N-oxide (S23). Following General Procedure $\mathrm{A}$, the title compound was prepared from 3,6-dichloropyridazine- $\mathrm{N}$-oxide $(800 \mathrm{mg}$, $4.85 \mathrm{mmol}$ ), $\mathbf{S} 10$ (1.12 g, $5.09 \mathrm{mmol}$ ) and $\mathrm{PdCl}_{2} \mathrm{dppf}(177 \mathrm{mg}, 0.24 \mathrm{mmol}, 5 \mathrm{~mol} \%$ ). After $1 \mathrm{~h}$, purification by flash chromatography $\left(\mathrm{SiO}_{2}, 1: 1\right.$ hexanes/EtOAc) provided S23 (668 mg, $3.00 \mathrm{mmol}, 62 \%$ yield) as a colorless solid: $\mathbf{m p}=192-195^{\circ} \mathrm{C}$; ${ }^{1} \mathbf{H}$ NMR $\left(400 \mathrm{MHz}, \mathrm{DMSO}-\mathrm{d}_{6}\right) \delta 10.49(\mathrm{~s}, 1 \mathrm{H}), 8.27(\mathrm{~d}, J=8.3 \mathrm{~Hz}, 1 \mathrm{H}), 7.85(\mathrm{~d}, J=8.3 \mathrm{~Hz}$, $1 \mathrm{H}), 7.67(\mathrm{t}, J=8.0 \mathrm{~Hz}, 1 \mathrm{H}), 7.35(\mathrm{t}, J=7.6 \mathrm{~Hz}, 1 \mathrm{H}), 7.02(\mathrm{~d}, J=8.3 \mathrm{~Hz}, 1 \mathrm{H}), 6.96(\mathrm{t}$, $J=7.6 \mathrm{~Hz}, 1 \mathrm{H}) ;{ }^{13} \mathbf{C ~ N M R}(150 \mathrm{MHz}$, DMSO-d 6 ) $\delta$ C: $156.9,156.5,135.3,120.4 ; \mathbf{C H}:$ 135.3, 132.5, 130.3, 120.1, 119.3, 117.3; IR (thin-film) 2924, 1322, $756 \mathrm{~cm}^{-1}$; HRMSESI $(m / z)[M+N a]^{+}$calculated for $\mathrm{C}_{10} \mathrm{H}_{7} \mathrm{ClN}_{2} \mathrm{O}_{2} \mathrm{Na}=245.0094$; found 245.0089 .

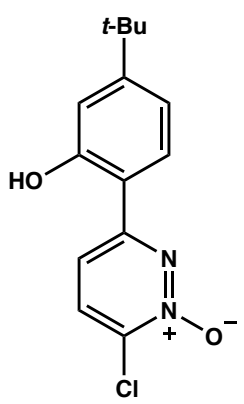

S24: $\mathrm{C}_{14} \mathrm{H}_{15} \mathrm{ClN}_{2} \mathrm{O}_{2}$ $\mathrm{MW}: 278.74$

3-(4-(tert-butyl)-2-hydroxyphenyl)-6-chloropyridazine-N-oxide (S24). Following a modification of General Procedure A, the title compound was prepared from 3,6dichloropyridazine- $N$-oxide $(450 \mathrm{mg}, 2.70 \mathrm{mmol}), \mathbf{S} 11(828 \mathrm{mg}, 3.00 \mathrm{mmol})$, and $\mathrm{PdCl}_{2} \mathrm{dppf} \cdot \mathrm{CH}_{2} \mathrm{Cl}_{2}(110 \mathrm{mg}, 0.14 \mathrm{mmol}, 5 \mathrm{~mol} \%)$. After $45 \mathrm{~min}$, the reaction mixture was diluted with $\mathrm{CH}_{2} \mathrm{Cl}_{2}(30 \mathrm{~mL})$. The reaction mixture was then washed with $1 \mathrm{M}$ $\mathrm{HCl}(5 \mathrm{~mL})$ and the aqueous layer was extracted with $\mathrm{CH}_{2} \mathrm{Cl}_{2}(3 \times 15 \mathrm{~mL})$. The combined organics were dried over $\mathrm{MgSO}_{4}$, filtered, and concentrated under reduced pressure. Purification by flash chromatography $\left(\mathrm{SiO}_{2}, 1: 1\right.$ hexanes/EtOAc) provided S24 (208 mg, $0.75 \mathrm{mmol}, 25 \%$ yield) as a colorless solid: $\mathbf{m p}=202-203$ ${ }^{\circ} \mathrm{C}$ (decomp); ${ }^{1} \mathbf{H}$ NMR $\left(400 \mathrm{MHz}, \mathrm{CDCl}_{3}\right) \delta 10.70(\mathrm{~s}, 1 \mathrm{H}), 7.85(\mathrm{~d}, J=8.8 \mathrm{~Hz}, 1 \mathrm{H})$, $7.59(\mathrm{~d}, J=8.5 \mathrm{~Hz}, 1 \mathrm{H}), 7.52(\mathrm{~d}, J=8.9 \mathrm{~Hz}, 1 \mathrm{H}), 7.10(\mathrm{~d}, J=2.0 \mathrm{~Hz}, 1 \mathrm{H}), 7.02$ (dd, $J=6.5,2.1 \mathrm{~Hz}, 1 \mathrm{H}), 1.33(\mathrm{~s}, 9 \mathrm{H}) ;{ }^{13} \mathrm{C}$ NMR $\left(150 \mathrm{MHz}, \mathrm{CDCl}_{3}\right) \delta \mathrm{C}: 158.6,158.2,158.1,134.6,112.5$, 35.0; CH: 134.9, 127.0, 117.8, 116.2, 113.5; $\mathbf{C H}_{3}$ : 30.9; IR (thin-film) 3050, 2955, $1581 \mathrm{~cm}^{-1}$; HRMSESI $(\mathrm{m} / \mathrm{z})[\mathrm{M}+\mathrm{H}]^{+}$calculated for $\mathrm{C}_{14} \mathrm{H}_{16} \mathrm{CIN}_{2} \mathrm{O}_{2}=279.0895$; found 279.0906 . 


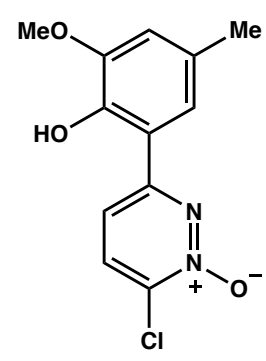

S25: $\mathrm{C}_{12} \mathrm{H}_{11} \mathrm{ClN}_{2} \mathrm{O}_{3}$ MW: 266.68

Following a modification of General Procedure A, the title compound was prepared from 3,6-dichloropyridazine- $N$-oxide (277 mg, $1.68 \mathrm{mmol}), \quad \mathbf{S 1 2}$ (630 mg, 2.52 $\mathrm{mmol}$ ), and $\mathrm{PdCl}_{2} \mathrm{dppf} \cdot \mathrm{CH}_{2} \mathrm{Cl}_{2}(69 \mathrm{mg}, 0.084 \mathrm{mmol}, 5 \mathrm{~mol} \%)$. After $1 \mathrm{~h}$, the reaction mixture was diluted with $\mathrm{CH}_{2} \mathrm{Cl}_{2}(30 \mathrm{~mL})$. The reaction mixture was then washed with $1 \mathrm{M} \mathrm{HCl}(5 \mathrm{~mL})$ and the aqueous layer was extracted with $\mathrm{CH}_{2} \mathrm{Cl}_{2}(3 \times 15 \mathrm{~mL})$. The combined organics were dried over $\mathrm{Na}_{2} \mathrm{SO}_{4}$, filtered, and concentrated under reduced pressure. Purification by flash chromatography $\left(\mathrm{SiO}_{2}, 4: 1\right.$ hexanes/acetone $)$ provided S25 (343 mg, $1.29 \mathrm{mmol}, 77 \%$ yield) as a yellow solid: $\mathbf{m p}=166-168{ }^{\circ} \mathrm{C}$;

${ }^{1} \mathrm{H}$ NMR (400 MHz, $\left.\mathrm{CDCl}_{3}\right) \delta 7.81-7.76(\mathrm{~m}, 3 \mathrm{H}), 7.29(\mathrm{~s}, 1 \mathrm{H}), 6.81(\mathrm{~s}, 1 \mathrm{H}), 3.92(\mathrm{~s}$, $3 \mathrm{H}), 2.33(\mathrm{~s}, 3 \mathrm{H}) ;{ }^{13} \mathrm{C}$ NMR (150 MHz, $\left.\mathrm{CDCl}_{3}\right) \delta \mathrm{C}: 157.1,147.7,144.0,135.4,129.5,117.3$; CH: 134.3, 120.6, 117.2, 114.7; $\mathbf{C H}_{3}: 56.3,21.1$; IR (thin-film) 3137, 1310, $779 \mathrm{~cm}^{-1}$; HRMS-DART (m/z) $\left[\mathrm{M}+\mathrm{NH}_{4}\right]^{+}$calculated for $\mathrm{C}_{12} \mathrm{H}_{15} \mathrm{CIN}_{3} \mathrm{O}_{3}=284.0786$; found 284.0785.

Preparation of substituted 3-aryl-6-(pyrrolidin-1-yl)pyridazine-N-oxides. General Procedure B. Following a modification of our reported procedure, ${ }^{6}$ 6-chloro-3-aryl-pyridazine- $N$-oxides S13-25 were suspended in freshly distilled pyrrolidine (12.0 equiv). The stirred slurry was warmed to $85^{\circ} \mathrm{C}$ until the reaction was judged to be complete by TLC (ca. 3-18 h). The reaction mixture was cooled to $\mathrm{rt}$ and concentrated under reduced pressure. The suspension was diluted with $\mathrm{CHCl}_{3}(30 \mathrm{~mL} / \mathrm{mmol})$ and washed with saturated aq. $\mathrm{NaHCO}_{3}(15 \mathrm{~mL} / \mathrm{mmol})$. The aqueous layer was then extracted with $\mathrm{CHCl}_{3}(2 \times 10 \mathrm{~mL} / \mathrm{mmol})$. The combined organics were dried with $\mathrm{Na}_{2} \mathrm{SO}_{4}$, filtered, and concentrated under reduced pressure. The resulting residue was purified by flash chromatography, trituration or recrystallization using the indicated solvents.

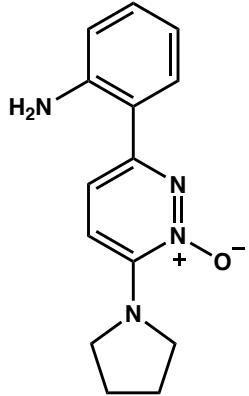

2b: $\mathrm{C}_{14} \mathrm{H}_{16} \mathrm{~N}_{4} \mathrm{O}$ MW: 256.31

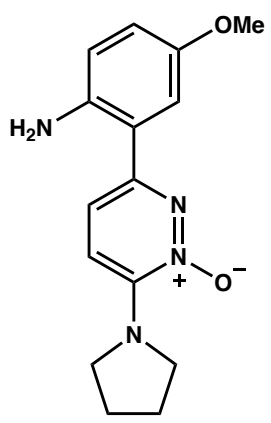

$2 b_{1}: \mathrm{C}_{15} \mathrm{H}_{18} \mathrm{~N}_{4} \mathrm{O}_{2}$ MW: 286.34
3-(2-aminophenyl)-6-(pyrrolidin-1-yl)pyridazine-N-oxide (2b). Following General Procedure $\mathrm{B}$, the title compound was prepared from $\mathbf{S 1 3}(1.00 \mathrm{~g}, 3.95 \mathrm{mmol})$ and pyrrolidine $(3.9 \mathrm{~mL}, 47.4 \mathrm{mmol})$. After $2 \mathrm{~h}$, purification by flash chromatography $\left(\mathrm{SiO}_{2}\right.$, 1:1 $\mathrm{CH}_{2} \mathrm{Cl}_{2}$ /acetone) provided $\mathbf{2 b}(901 \mathrm{mg}, 3.52 \mathrm{mmol}, 89 \%$ yield) as a yellow solid: $\mathrm{mp}=182-183{ }^{\circ} \mathrm{C} ;{ }^{1} \mathrm{H}$ NMR $\left(400 \mathrm{MHz}, \mathrm{CDCl}_{3}\right) \delta 7.38(\mathrm{~d}, \mathrm{~J}=7.5 \mathrm{~Hz}, 1 \mathrm{H}), 7.33(\mathrm{~d}, \mathrm{~J}=$ $8.1 \mathrm{~Hz}, 1 \mathrm{H}), 7.16(\mathrm{t}, J=7.3 \mathrm{~Hz}, 1 \mathrm{H}), 7.06(\mathrm{~d}, J=8.5 \mathrm{~Hz}, 1 \mathrm{H}), 6.77-6.72(\mathrm{~m}, 2 \mathrm{H}), 5.42$ (br s, 2H), 3.71-3.68 (m, 4H), 2.01-1.98 (m, 4H); $\left.{ }^{13} \mathbf{C ~ N M R ~ ( 1 5 0 ~ M H z , ~ C D C l ~} 3\right) \delta$ C: 150.4, 146.0, 145.4, 117.7; CH: 130.3, 128.5, 119.5, 117.9, 117.5, 117.3; CH$_{2}: 49.9$, 25.2; IR (thin-film) 3406, 1341, $1280 \mathrm{~cm}^{-1}$; HRMS-ESI $(\mathrm{m} / \mathrm{z})[\mathrm{M}+\mathrm{H}]^{+}$calculated for $\mathrm{C}_{14} \mathrm{H}_{17} \mathrm{~N}_{4} \mathrm{O}=257.1397 ;$ found 257.1407.

3-(2-amino-5-methoxyphenyl)-6-(pyrrolidin-1-yl)pyridazine- $N$-oxide $\left(2 b_{1}\right)$. Following a modification of General Procedure B, the title compound was prepared from S14 (257 mg, $1.00 \mathrm{mmol})$, pyrrolidine $(0.4 \mathrm{~mL}, 5.0 \mathrm{mmol})$ and $\mathrm{K}_{2} \mathrm{CO}_{3}(415 \mathrm{mg}, 3.00$ $\mathrm{mmol})$.. After $6 \mathrm{~h}$, purification by flash chromatography $\left(\mathrm{SiO}_{2}, 1: 1\right.$ hexanes/acetone) provided 2 $\mathbf{b}_{\mathbf{1}}$ (269 mg, $0.94 \mathrm{mmol}, 94 \%$ yield) as a yellow solid: $\mathbf{m p :} 183-185{ }^{\circ} \mathrm{C} ;{ }^{1} \mathbf{H}$ NMR $\left(400 \mathrm{MHz} \mathrm{CDCl}_{3}\right) \delta 7.30(\mathrm{~d}, J=8.8 \mathrm{~Hz}, 1 \mathrm{H}), 7.06(\mathrm{~d}, J=8.8 \mathrm{~Hz}, 1 \mathrm{H}), 6.93(\mathrm{br}$ $\mathrm{d}, J=2.8 \mathrm{~Hz}, 1 \mathrm{H}), 6.81(\mathrm{dd}, J=8.7,5.9 \mathrm{~Hz}, 1 \mathrm{H}), 6.70(\mathrm{~d}, J=8.8 \mathrm{~Hz}, 1 \mathrm{H}), 4.96(\mathrm{br} \mathrm{s}$, $2 \mathrm{H}), 3.78(\mathrm{~s}, 3 \mathrm{H}), 3.71-3.68(\mathrm{~m}, 4 \mathrm{H}), 2.01-1.98(\mathrm{~m}, 4 \mathrm{H}) ;{ }^{13} \mathrm{C}$ NMR $\left(150 \mathrm{MHz}, \mathrm{CDCl}_{3}\right)$ $\delta$ C: 152.0, 150.0, 145.7, 139.7, 119.1; CH: 119.3, 118.5, 118.0, 116.7, 113.9; $\mathbf{C H}_{2}$ : 50.0, 25.2; $\mathbf{C H}_{3}$ : 56.0; IR (thin-film) 3403, 3276, $1338 \mathrm{~cm}^{-1}$; HRMS-ESI (m/z) [M+H] $]^{+}$ calculated for $\mathrm{C}_{15} \mathrm{H}_{19} \mathrm{~N}_{4} \mathrm{O}_{2}=287.1503$; found 287.1517 . 


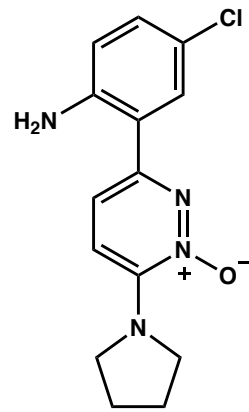

$2 \mathbf{b}_{2}: \mathrm{C}_{14} \mathrm{H}_{15} \mathrm{CIN}_{4} \mathrm{O}$ MW: 290.75

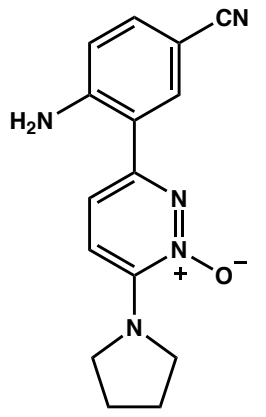

$2 b_{3}: \mathrm{C}_{15} \mathrm{H}_{15} \mathrm{~N}_{5} \mathrm{O}$ MW: 281.32

3-(2-amino-5-chlorophenyl)-6-(pyrrolidin-1-yl)pyridazine- $N$-oxide $\quad\left(2 b_{2}\right)$. Following General Procedure B, the title compound was prepared from S15 (175 mg, 0.69 $\mathrm{mmol}$ ) and pyrrolidine $(0.7 \mathrm{~mL}, 8.3 \mathrm{mmol})$. After $1 \mathrm{~h}$, purification by flash chromatography $\left(\mathrm{SiO}_{2}, 3: 3: 4 \mathrm{CH}_{2} \mathrm{Cl}_{2}\right.$ /hexane/acetone) provided $\mathbf{2 b}_{\mathbf{2}}$ (186 $\mathrm{mg}, 0.64$ mmol, 93\% yield) as a yellow solid: $\mathbf{m p}=188-190{ }^{\circ} \mathrm{C} ;{ }^{1} \mathbf{H} \mathbf{N M R}\left(400 \mathrm{MHz}, \mathrm{CDCl}_{3}\right) \delta$ $7.35(\mathrm{~d}, J=2.6 \mathrm{~Hz}, 1 \mathrm{H}), 7.29(\mathrm{~d}, J=8.9 \mathrm{~Hz}, 1 \mathrm{H}), 7.10(\mathrm{dd}, J=8.8,2.7 \mathrm{~Hz}, 1 \mathrm{H}), 7.06$ $(\mathrm{d}, J=8.9 \mathrm{~Hz}, 1 \mathrm{H}), 6.67(\mathrm{~d}, J=8.7 \mathrm{~Hz}, 1 \mathrm{H}), 5.45(\mathrm{br} \mathrm{s}, 2 \mathrm{H}), 3.73-3.71(\mathrm{~m}, 4 \mathrm{H}), 2.02-$ $2.00(\mathrm{~m}, 4 \mathrm{H}) ;{ }^{13} \mathrm{C}$ NMR (150 MHz, $\left.\mathrm{CDCl}_{3}\right) \delta \mathrm{C}: 149.0,145.7,144.5,122.0,118.8 ; \mathbf{C H}:$ 130.0, 127.9, 119.2, 118.5, 117.7; $\mathbf{C H}_{2}: 50.0$, 25.3; IR (thin-film) 3384, 1339, $754 \mathrm{~cm}^{-}$ 1; HRMS-ESI $(\mathrm{m} / \mathrm{z})[\mathrm{M}+\mathrm{H}]^{+}$calculated for $\mathrm{C}_{14} \mathrm{H}_{16} \mathrm{CIN}_{4} \mathrm{O}=291.1007$; found 291.0993.

3-(2-amino-5-cyanophenyl)-6-(pyrrolidin-1-yl)pyridazine- $N$-oxide (2b 3$)$. Following a modification of General Procedure B, the title compound was prepared from S16 (600 $\mathrm{mg}, 2.43 \mathrm{mmol})$, pyrrolidine $(0.6 \mathrm{~mL}, 7.3 \mathrm{mmol})$, and $\mathrm{K}_{2} \mathrm{CO}_{3}(1.01 \mathrm{~g}, 7.30 \mathrm{mmol})$. After $16 \mathrm{~h}$, the reaction was cooled to rt, filtered, and concentrated under reduced pressure. The resulting solid was purified by trituration with cold $\mathrm{CHCl}_{3}(5 \mathrm{~mL})$ and cyclohexane (5 mL) to give $\mathbf{2 b}_{\mathbf{3}}(629 \mathrm{mg}, 0.82 \mathrm{mmol}, 92 \%$ yield) as a yellow solid: $\mathbf{m p}=240-242$ ${ }^{\circ} \mathrm{C} ;{ }^{1} \mathrm{H}$ NMR $\left(400 \mathrm{MHz} \mathrm{CDCl}_{3}\right) \delta 7.70(\mathrm{~d}, J=1.7 \mathrm{~Hz}, 1 \mathrm{H}), 7.37$ (dd, $J=6.6,2.4 \mathrm{~Hz}$, $1 \mathrm{H}), 7.34(\mathrm{~d}, J=8.7 \mathrm{~Hz}, 1 \mathrm{H}), 7.10(\mathrm{~d}, J=8.8 \mathrm{~Hz}, 1 \mathrm{H}), 6.73(\mathrm{~d}, J=8.7 \mathrm{~Hz}, 1 \mathrm{H}), 6.30$ (br s, 2H), 3.76-3.73 (m, 4H), 2.04-2.00 (m, 4H); $\left.{ }^{13} \mathbf{C ~ N M R ~ ( 1 5 0 ~ M H z , ~} \mathrm{CDCl}_{3}\right) \delta$ C: 149.7, 148.1, 145.8, 119.9, 116.8, 99.2; CH: 133.3, 132.9, 119.4, 117.3, 117.2; $\mathbf{C H}_{2}$ : 50.1, 25.3; IR (thin-film) 3371, 2955, $2214 \mathrm{~cm}^{-1}$; HRMS-ESI $(\mathrm{m} / \mathrm{z})[\mathrm{M}+\mathrm{H}]^{+}$calculated for $\mathrm{C}_{15} \mathrm{H}_{16} \mathrm{~N}_{5} \mathrm{O}=282.1349$; found 282.1349.

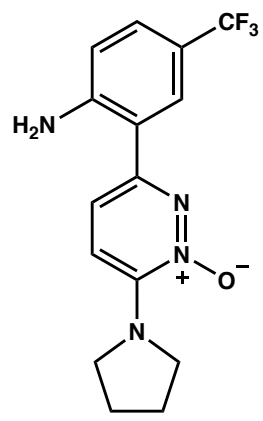

$2 \mathbf{b}_{4}: \mathrm{C}_{15} \mathrm{H}_{15} \mathrm{~F}_{3} \mathrm{~N}_{4} \mathrm{O}$ MW: 324.31
3-(2-amino-5-(trifluoromethyl)phenyl)-6-(pyrrolidin-1-yl)pyridazine-N-oxide Following a modification of General Procedure B, the title compound was prepared from S17 (621 mg, $2.15 \mathrm{mmol})$, pyrrolidine $(0.4 \mathrm{~mL}, 4.3 \mathrm{mmol})$, and $\mathrm{K}_{2} \mathrm{CO}_{3}(891 \mathrm{mg}$, $6.45 \mathrm{mmol}$ ). After $13 \mathrm{~h}$, was cooled to $\mathrm{rt}$, filtered, and concentrated under reduced pressure. The resulting solid was purified by trituration with THF $(5 \mathrm{~mL})$ and cyclohexane $(5 \mathrm{~mL})$ to give $\mathbf{2} \mathbf{b}_{\mathbf{4}}(601 \mathrm{mg}, 1.85 \mathrm{mmol}, \mathbf{8 6} \%$ yield) as a yellow solid: $\mathbf{m p}=212-213{ }^{\circ} \mathrm{C} ;{ }^{1} \mathrm{H}$ NMR $\left(400 \mathrm{MHz} \mathrm{CDCl}_{3}\right) \delta 7.64(\mathrm{br} \mathrm{s}, 1 \mathrm{H}), 7.37(\mathrm{~d}, J=8.9 \mathrm{~Hz}$, $2 \mathrm{H}), 7.08(\mathrm{~d}, J=8.9 \mathrm{~Hz}, 1 \mathrm{H}), 6.77(\mathrm{~d}, J=8.5 \mathrm{~Hz}, 1 \mathrm{H}), 5.91(\mathrm{br} \mathrm{s}, 2 \mathrm{H}), 3.75-3.72(\mathrm{~m}$, 4H), 2.03-2.00 (m, 4H); ${ }^{13} \mathrm{C}$ NMR (150 MHz, $\left.\mathrm{CDCl}_{3}\right) \delta \mathrm{C:}: 149.0,148.7,145.7,124.8$ (d, J = $271 \mathrm{~Hz}, 119.0$ (d, J = $33 \mathrm{~Hz}), 116.4$; CH: 127.0 (d, J = $3 \mathrm{~Hz}), 125.7(\mathrm{~d}, J=4$ $\mathrm{Hz}$ ), 119.4, 117.7, 116.9; $\mathbf{C H}_{2}$ : 50.1, 25.3; IR (thin-film) 2965, 1245, $1140 \mathrm{~cm}^{-1}$; HRMS-ESI $(\mathrm{m} / \mathrm{z})[\mathrm{M}+\mathrm{H}]^{+}$calculated for $\mathrm{C}_{15} \mathrm{H}_{16} \mathrm{~F}_{3} \mathrm{~N}_{4} \mathrm{O}=325.1271$; found 325.1283.

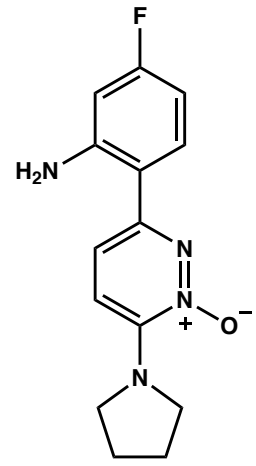

$2 b_{5}: \mathrm{C}_{14} \mathrm{H}_{15} \mathrm{FN}_{4} \mathrm{O}$ MW: 274.30

3-(2-amino-4-fluorophenyl)-6-(pyrrolidin-1-yl)pyridazine- $N$-oxide $\quad\left(2 b_{5}\right)$. Following General Procedure B, the title compound was prepared from S18 $(220 \mathrm{mg}, 0.92$ $\mathrm{mmol})$ and pyrrolidine $(1.2 \mathrm{~mL}, 15 \mathrm{mmol})$. After $22 \mathrm{~h}$, purification by flash chromatography $\left(\mathrm{SiO}_{2}\right.$, acetone) provided $\mathbf{2} \mathbf{b}_{5}(184 \mathrm{mg}, 0.67 \mathrm{mmol}, 73 \%$ yield) as a yellow-green solid: $\mathbf{m p}$ : $180-181^{\circ} \mathrm{C} ;{ }^{1} \mathbf{H}$ NMR $\left(600 \mathrm{MHz}, \mathrm{CDCl}_{3}\right) \delta 7.35(\mathrm{t}, J=7.7 \mathrm{~Hz}$, $1 \mathrm{H}), 7.28(\mathrm{~d}, J=7.4 \mathrm{~Hz}, 1 \mathrm{H}), 7.08(\mathrm{~d}, J=7.0 \mathrm{~Hz}, 1 \mathrm{H}), 6.46-6.41(\mathrm{~m}, 2 \mathrm{H}), 5.40$ (br s, 2H), $3.72(\mathrm{~m}, 4 \mathrm{H}), 2.01(\mathrm{~m}, 4 \mathrm{H}) ;{ }^{13} \mathrm{C}$ NMR (150 MHz, $\left.\mathrm{CDCl}_{3}\right) \delta \mathrm{C}: 164.2(\mathrm{~d}, \mathrm{~J}=245$ $\mathrm{Hz}), 149.8,147.9$ (d, J = $11 \mathrm{~Hz}), 145.4,114.0 ; \mathbf{C H}: 130.3$ (d, J = $11 \mathrm{~Hz}), 120.0,117.8$, 104.8 (d, $J=22 \mathrm{~Hz}, 103.2$ (d, $J=24 \mathrm{~Hz}$ ); $\mathbf{C H}_{2}$ : 50.0, 25.3; IR (thin-film) 3395, 2969, $1339 \mathrm{~cm}^{-1}$; HRMS-DART $(\mathrm{m} / \mathrm{z})[\mathrm{M}+\mathrm{H}]^{+}$calculated for $\mathrm{C}_{14} \mathrm{H}_{16} \mathrm{FN}_{4} \mathrm{O}=275.1303$; found 275.1316. 


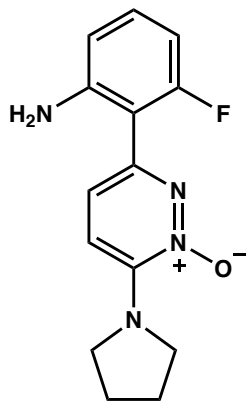

$2 \mathbf{b}_{6}: \mathrm{C}_{14} \mathrm{H}_{15} \mathrm{FN}_{4} \mathrm{O}$ MW: 274.30

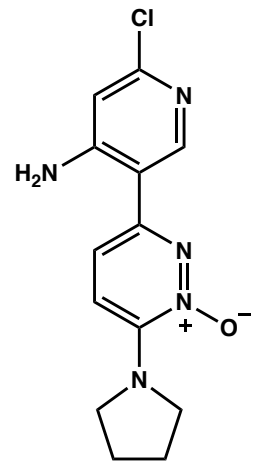

$2 b_{7}: \mathrm{C}_{13} \mathrm{H}_{14} \mathrm{CIN}_{5} \mathrm{O}$ MW: 291.74

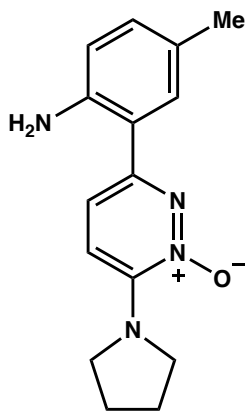

$2 \mathbf{b}_{8}: \mathrm{C}_{15} \mathrm{H}_{18} \mathrm{~N}_{4} \mathrm{O}$ MW: 270.34

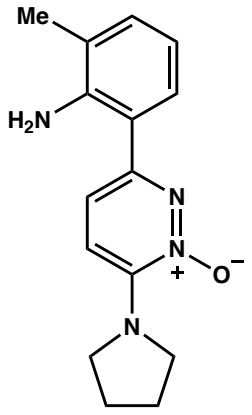

2b $\mathbf{b}_{9}: \mathrm{C}_{15} \mathrm{H}_{18} \mathrm{~N}_{4} \mathrm{O}$ MW: 270.34

3-(2-amino-6-fluorophenyl)-6-(pyrrolidin-1-yl)pyridazine- $N$-oxide $\quad\left(2 b_{6}\right)$. Following General Procedure B, the title compound was prepared from S19 (110 mg, 0.46 $\mathrm{mmol})$ and pyrrolidine $(0.5 \mathrm{~mL}, 5.5 \mathrm{mmol})$. After $6 \mathrm{~h}$, purification by flash chromatography $\left(\mathrm{SiO}_{2}, 1: 1 \rightarrow 1: 3\right.$ hexane/acetone) provided $\mathbf{2} \mathbf{b}_{\mathbf{6}}(103 \mathrm{mg}, 0.46 \mathrm{mmol}$, $82 \%$ yield) as a yellow solid: $\mathrm{mp}$ : $182-183{ }^{\circ} \mathrm{C} ;{ }^{1} \mathrm{H}$ NMR $\left(600 \mathrm{MHz} \mathrm{CDCl}_{3}\right) \delta 7.27-$ $7.25(\mathrm{~m}, 1 \mathrm{H}), 7.03(\mathrm{t}, J=8.7 \mathrm{~Hz}, 2 \mathrm{H}), 6.51(\mathrm{~d}, J=7.9 \mathrm{~Hz}, 1 \mathrm{H}), 6.45(\mathrm{t}, J=8.9 \mathrm{~Hz}, 1 \mathrm{H})$, 5.12 (br s, 2H), $3.70(\mathrm{~m}, 4 \mathrm{H}), 1.98(\mathrm{~m}, 4 \mathrm{H}) ;{ }^{13} \mathrm{C}$ NMR $\left(150 \mathrm{MHz}, \mathrm{CDCl}_{3}\right) \delta \mathrm{C}: 161.4(\mathrm{~d}$, $J=245), 147.4$ (d, $J=5), 146.0,144.7,108.0$ (d, $J=16) ; C H: 130.5$ (d, J = 11), 121.8 (d, $J=9$ ), 118.8, 112.3 (d, $J=2$ ), 104.4 (d, $J=24) ; \mathbf{C H}_{2}: 50.0,25.3$; IR (thin-film) 3461, 2968, $1338 \mathrm{~cm}^{-1}$; HRMS-DART $(\mathrm{m} / \mathrm{z})[\mathrm{M}+\mathrm{H}]^{+}$calculated for $\mathrm{C}_{14} \mathrm{H}_{16} \mathrm{FN}_{4} \mathrm{O}=$ 275.1303; found 275.1309.

3-(4-amino-6-chloropyridin-3-yl)-6-(pyrrolidin-1-yl)pyridazine-N-oxide (2 $\left.\mathrm{b}_{7}\right)$. Following a modification of General Procedure B, the title compound was prepared from $\mathbf{S 2 0}$ (400 mg, $1.56 \mathrm{mmol})$, pyrrolidine $(0.25 \mathrm{~mL}, 3.04 \mathrm{mmol})$, and $\mathrm{K}_{2} \mathrm{CO}_{3}(647 \mathrm{mg}, 4.68$ $\mathrm{mmol}$ ). After $18 \mathrm{~h}$, the reaction was cooled to rt, filtered, and concentrated. The resulting residue was purified by flash chromatography $\left(\mathrm{SiO}_{2}\right.$, acetone) to give $\mathbf{2} \mathbf{b}_{7}$ (301 mg, $1.03 \mathrm{mmol}, 66 \%$ yield) as a yellow solid: mp: $171-173{ }^{\circ} \mathrm{C} ;{ }^{1} \mathbf{H}$ NMR (400 $\left.\mathrm{MHz} \mathrm{CDCl}_{3}\right) \delta 8.32(\mathrm{~s}, 1 \mathrm{H}), 7.38(\mathrm{~d}, J=8.9 \mathrm{~Hz}, 1 \mathrm{H}), 7.08(\mathrm{~d}, J=9.0 \mathrm{~Hz}, 1 \mathrm{H}), 6.61(\mathrm{~s}$, $1 \mathrm{H}), 6.28$ (br s, 2H), 3.76-3.72 (m, 4H), 2.04-2.00 (m, 4H); ${ }^{13} \mathrm{C}$ NMR $(150 \mathrm{MHz}$, $\left.\mathrm{CDCl}_{3}\right) \delta$ C: $153.3,152.0,147.0,145.9,112.8 ; \mathbf{C H}: 148.5,119.4,116.7,109.6 ; \mathbf{C H}_{2}$ : 50.1, 25.3; IR (thin-film) 3376, 1556, $1345 \mathrm{~cm}^{-1}$; HRMS-ESI (m/z) [M+H] ${ }^{+}$calculated for $\mathrm{C}_{13} \mathrm{H}_{15} \mathrm{CIN}_{5} \mathrm{O}=292.0960$; found 292.0952 .

3-(2-amino-5-methylphenyl)-6-(pyrrolidin-1-yl)pyridazine-N-oxide (2b 8 . Following a modification of General Procedure B, the title compound was prepared from S21 (372 $\mathrm{mg}, 1.62 \mathrm{mmol})$, pyrrolidine $(1.6 \mathrm{~mL}, 20 \mathrm{mmol})$ and THF $(2 \mathrm{~mL})$. After $1 \mathrm{~h}$, purification by recrystallization (PhMe) provided $\mathbf{2 b}_{\mathbf{8}}(372 \mathrm{mg}, 1.40 \mathrm{mmol}, 86 \%$ yield) as a yellow solid: mp: $201-203{ }^{\circ} \mathrm{C} ;{ }^{1} \mathrm{H}$ NMR $\left(400 \mathrm{MHz} \mathrm{CDCl}_{3}\right) \delta 7.32$ (d, J=8.9 Hz, 1H), 7.18 (s, $1 \mathrm{H}), 7.05(\mathrm{~d}, J=8.9 \mathrm{~Hz}, 1 \mathrm{H}), 6.98(\mathrm{~d}, J=8.1 \mathrm{~Hz}, 1 \mathrm{H}), 6.66(\mathrm{~d}, J=8.1 \mathrm{~Hz}, 1 \mathrm{H}), 5.09$ (br s, 2H), 3.71-3.68 (m, 4H), $2.26(\mathrm{~s}, 3 \mathrm{H}), 2.01-1.98(\mathrm{~m}, 4 \mathrm{H}) ;{ }^{13} \mathrm{C}$ NMR (150 MHz, DMSO-d 6 ) $\delta$ C: 150.5, 145.6, 144.7, 125.0, 117.0; CH: 131.2, 129.1, 120.8, 117.7, 117.2; $\mathbf{C H}_{2}: 49.7,25.1 ; \mathbf{C H}_{3}: 20.5$; IR (thin-film) 3395, 2912, $1339 \mathrm{~cm}^{-1}$; HRMS-ESI $(\mathrm{m} / \mathrm{z})[\mathrm{M}+\mathrm{H}]^{+}$calculated for $\mathrm{C}_{15} \mathrm{H}_{19} \mathrm{~N}_{4} \mathrm{O}=271.1562$; found 271.1562.

3-(2-amino-3-methylphenyl)-6-(pyrrolidin-1-yl)pyridazine-N-oxide $\quad\left(2 b_{9}\right)$. Following General Procedure B, the title compound was prepared from S22 (200 mg, 0.85 $\mathrm{mmol})$ and pyrrolidine $(0.8 \mathrm{~mL}, 10.2 \mathrm{mmol})$. After $0.5 \mathrm{~h}$, purification by flash chromatography $\left(\mathrm{SiO}_{2}, 1: 1\right.$ hexane/acetone) provided 2 $\mathbf{b}_{9}(201 \mathrm{mg}, 0.74 \mathrm{mmol}, 87 \%$ yield) as a yellow solid: $\mathrm{mp:} 192-194{ }^{\circ} \mathrm{C}$; ${ }^{1} \mathrm{H}$ NMR $\left(400 \mathrm{MHz} \mathrm{CDCl}_{3}\right) \delta 7.32(\mathrm{~d}, \mathrm{~J}=8.9$ $\mathrm{Hz}, 1 \mathrm{H}), 7.27(\mathrm{~d}, J=7.4 \mathrm{~Hz}, 1 \mathrm{H}), 7.08(\mathrm{t}, J=9.1 \mathrm{~Hz}, 2 \mathrm{H}), 6.69(\mathrm{t}, J=7.5 \mathrm{~Hz}, 1 \mathrm{H}), 5.42$ (br s, 2H), 3.72-3.69 (m, 4H), $2.21(\mathrm{~s}, 3 \mathrm{H}), 2.02-1.99(\mathrm{~m}, 4 \mathrm{H}) ;{ }^{13} \mathrm{C}$ NMR $(150 \mathrm{MHz}$, $\left.\mathrm{CDCl}_{3}\right) \delta \mathbf{C :} 150.9,145.4,144.1,123.5,117.4 ; \mathbf{C H}: 131.4,126.7,119.5,118.3,117.0$; $\mathbf{C H}_{2}$ : 49.9, 25.3; $\mathbf{C H}_{3}$ : 17.9; IR (thin-film) 3409, 2954, $1338 \mathrm{~cm}^{-1} ;$ HRMS-ESI (m/z) $[\mathrm{M}+\mathrm{H}]^{+}$calculated for $\mathrm{C}_{15} \mathrm{H}_{19} \mathrm{~N}_{4} \mathrm{O}=271.1553$; found 271.1557 . 


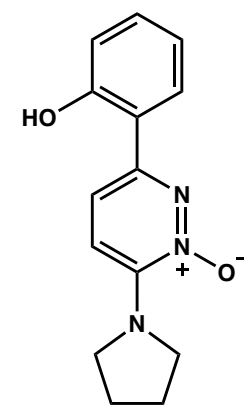

2c: $\mathrm{C}_{14} \mathrm{H}_{15} \mathrm{~N}_{3} \mathrm{O}_{2}$ MW: 257.29

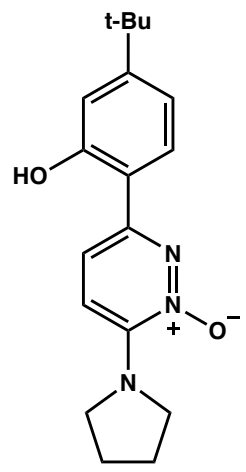

$2 \mathrm{c}_{1}: \mathrm{C}_{18} \mathrm{H}_{23} \mathrm{~N}_{3} \mathrm{O}_{2}$ MW: 313.40

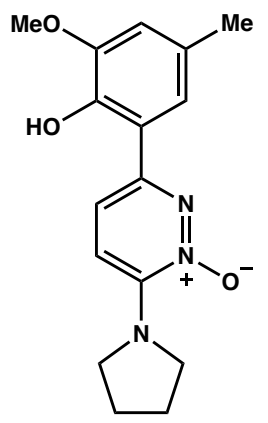

2c $\mathrm{c}_{2}: \mathrm{C}_{16} \mathrm{H}_{19} \mathrm{~N}_{3} \mathrm{O}_{3}$ MW: 301.35

3-(2-hydroxyphenyl)-6-(pyrrolidin-1-yl)pyridazine-N-oxide (2c). Following General Procedure B, the title compound was prepared from $\mathbf{S 2 3}$ (434 $\mathrm{mg}, 1.95 \mathrm{mmol})$ and pyrrolidine $(1.9 \mathrm{~mL}, 23 \mathrm{mmol})$. After $11 \mathrm{~h}$, the reaction was filtered and washed with cold THF (5 mL) to provide $2 \mathrm{c}(470 \mathrm{mg}, 1.83 \mathrm{mmol}, 94 \%$ yield) as a colorless solid: $\mathrm{mp}$ $=199-200{ }^{\circ} \mathrm{C} ;{ }^{1} \mathrm{H}$ NMR $\left(400 \mathrm{MHz}, \mathrm{CDCl}_{3}\right) \delta 11.16(\mathrm{~s}, 1 \mathrm{H}), 7.61(\mathrm{~d}, J=7.9 \mathrm{~Hz}, 1 \mathrm{H})$, $7.54(\mathrm{~d}, J=8.9 \mathrm{~Hz}, 1 \mathrm{H}), 7.32(\mathrm{t}, J=7.5 \mathrm{~Hz}, 1 \mathrm{H}), 7.13(\mathrm{~d}, J=8.9 \mathrm{~Hz}, 1 \mathrm{H}), 7.07(\mathrm{t}, J=$ $8.2 \mathrm{~Hz}, 1 \mathrm{H}), 6.95(\mathrm{t}, J=7.6 \mathrm{~Hz}, 1 \mathrm{H}), 3.76(\mathrm{~m}, 4 \mathrm{H}), 2.04(\mathrm{~m}, 4 \mathrm{H}) ;{ }^{13} \mathrm{C}$ NMR $(150 \mathrm{MHz}$, $\left.\mathrm{CDCl}_{3}\right) \delta \mathrm{C}: 157.3,149.2,145.6,116.7$; CH: 131.4, 126.1, 119.7, 119.5, 118.6, 115.7; $\mathbf{C H}_{2}$ : 50.1, 25.3; IR (thin-film) 2972, 1232, $1138 \mathrm{~cm}^{-1}$; HRMS-ESI (m/z) [M+Na] calculated for $\mathrm{C}_{14} \mathrm{H}_{15} \mathrm{~N}_{3} \mathrm{O}_{2} \mathrm{Na}=280.1062$; found 280.1057 .

3-(4-(tert-butyl)-2-hydroxyphenyl)-6-(pyrrolidin-1-yl)pyridazine-N-oxide (2c 1 ). Following General Procedure B, the title compound was prepared from S24 (56 $\mathrm{mg}, 0.20 \mathrm{mmol}$ ) and pyrrolidine $(0.2 \mathrm{~mL}, 2 \mathrm{mmol})$. After $1 \mathrm{~h}$, purification by flash chromatography $\left(\mathrm{SiO}_{2}, 3: 1 \mathrm{CH}_{2} \mathrm{Cl}_{2} / \mathrm{Et}_{2} \mathrm{O}\right)$ provided $2 \mathrm{c}_{1}(48 \mathrm{mg}, 0.15 \mathrm{mmol}, 76 \%$ yield $)$ as a yellow solid: $\mathbf{m p}=210-213{ }^{\circ} \mathrm{C} ;{ }^{1} \mathrm{H}$ NMR $\left(400 \mathrm{MHz} \mathrm{CDCl}_{3}\right) \delta 11.10(\mathrm{~s}, 1 \mathrm{H}), 7.52(\mathrm{~d}, \mathrm{~J}=8.4 \mathrm{~Hz}$, $1 \mathrm{H}), 7.49(\mathrm{~d}, J=9.1 \mathrm{~Hz}, 1 \mathrm{H}), 7.10-7.07(\mathrm{~m}, 2 \mathrm{H}), 7.00(\mathrm{dd}, J=6.4,2.0 \mathrm{~Hz}, 1 \mathrm{H}), 3.74-$ $3.71(\mathrm{~m}, 4 \mathrm{H}), 2.02-1.99(\mathrm{~m}, 4 \mathrm{H}), 1.31(\mathrm{~s}, 9 \mathrm{H}) ;{ }^{13} \mathrm{C}$ NMR $\left(150 \mathrm{MHz}, \mathrm{CDCl}_{3}\right) \delta \mathrm{C:} 157.1$, 155.4, 149.4, 145.4, 113.9, 34.8; CH: 125.7, 119.8, 117.0, 115.6, 115.3; $\mathbf{C H}_{2}: 50.0$, 25.3; $\mathbf{C H}_{3}$ : 31.0; IR (thin-film) 3053, 2950, $1287 \mathrm{~cm}^{-1} ;$ HRMS-ESI (m/z) [M+H] calculated for $\mathrm{C}_{18} \mathrm{H}_{24} \mathrm{~N}_{3} \mathrm{O}_{2}=314.1863$; found 314.1876.

\section{Optimized procedures from Table 1.}

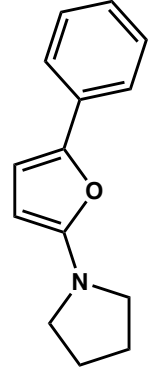

5a: $\mathrm{C}_{14} \mathrm{H}_{15} \mathrm{NO}$ MW: 213.28

1-(5-phenylfuran-2-yl)pyrrolidine (5a, Table 1, entry 5). A 1-dram vial was charged with pyridazine $\mathrm{N}$-oxide $2 \mathrm{a}(48 \mathrm{mg}, 0.20 \mathrm{mmol})$ and $\mathrm{Rh}_{2} \mathrm{esp}_{2}(2 \mathrm{mg}, 0.003 \mathrm{mmol}, 1.5 \mathrm{~mol} \%$ ). The reactants were suspended in THF $(0.7 \mathrm{~mL})$. The vial was capped under ambient atmosphere, transferred to a Rayonet chamber photoreactor, and irradiated ( $\mathrm{hv}=350$ $\mathrm{nm}$ ) at $35{ }^{\circ} \mathrm{C}$. After $3 \mathrm{~h}$, the reaction mixture was cooled to $\mathrm{rt}$ and concentrated under reduced pressure. The resulting residue was suspended in hexanes $(3 \mathrm{~mL})$. The slight yellow solution was decanted from red/brown solids. This process was repeated three additional times. The combined hexanes extracts were concentrated to give 5 a $(37 \mathrm{mg}$, $0.17 \mathrm{mmol}, 82 \%$ yield) as an amber oil. No further purification was necessary: ${ }^{1} \mathbf{H}$ NMR $\left(400 \mathrm{MHz}, \mathrm{CDCl}_{3}\right) \delta 7.50(\mathrm{~d}, J=7.3 \mathrm{~Hz}, 2 \mathrm{H}), 7.30(\mathrm{t}, J=8.1 \mathrm{~Hz}, 2 \mathrm{H}), 7.09$ (t, J= $7.4 \mathrm{~Hz}$, $1 \mathrm{H}), 6.58(\mathrm{~d}, J=3.3 \mathrm{~Hz}, 1 \mathrm{H}), 4.99(\mathrm{~d}, J=3.3 \mathrm{~Hz}, 1 \mathrm{H}), 3.37$ (br t, $J=6.6 \mathrm{~Hz}, 4 \mathrm{H}$ ), 1.99 (quint, $J=3.4$ $\mathrm{Hz}, 4 \mathrm{H})$; These and other characterization data were identical to those previously reported. ${ }^{6}$ 


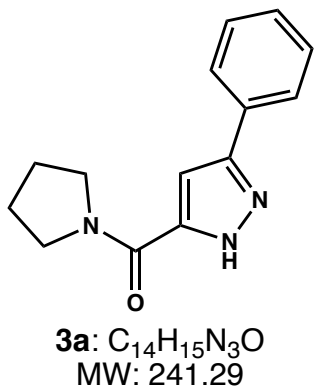

(3-phenyl-1H-pyrazol-5-yl)(pyrrolidine-1-yl)methanone (3a). ${ }^{1} \mathbf{H}$ NMR $(400 \mathrm{MHz}$, $\left.\mathrm{CDCl}_{3}\right) \delta 12.27(\mathrm{br} \mathrm{s}, 1 \mathrm{H}), 7.81(\mathrm{~d}, J=7.3 \mathrm{~Hz}, 2 \mathrm{H}), 7.41(\mathrm{t}, J=7.7 \mathrm{~Hz}, 2 \mathrm{H}), 7.33(\mathrm{t}$, $J=7.3 \mathrm{~Hz}, 1 \mathrm{H}), 6.91(\mathrm{~s}, 1 \mathrm{H}), 3.87(\mathrm{t}, J=6.8 \mathrm{~Hz}, 2 \mathrm{H}), 3.72(\mathrm{t}, J=6.8 \mathrm{~Hz}, 2 \mathrm{H}), 2.05$ (quint, $J=6.6 \mathrm{~Hz}, 2 \mathrm{H}$ ), 1.94 (quint, $J=6.7 \mathrm{~Hz}, 2 \mathrm{H}$ ); These and other characterization data were identical to those previously reported. ${ }^{6}$

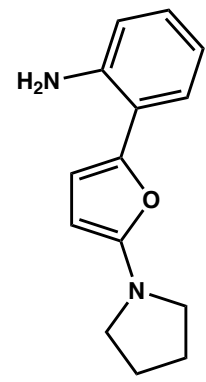

5b: $\mathrm{C}_{14} \mathrm{H}_{16} \mathrm{~N}_{2} \mathrm{O}$ MW: 228.30 2-(5-(pyrrolidin-1-yl)furan-2-yl)aniline (5b, Table 1, entry 9). A 1-dram vial was charged with pyridazine $N$-oxide $2 \mathrm{~b}(51 \mathrm{mg}, 0.20 \mathrm{mmol}), \mathrm{Rh}_{2} \mathrm{esp}_{2}(4.5 \mathrm{mg}, 0.006 \mathrm{mmol}, 3 \mathrm{~mol} \%)$ and powdered ${ }^{8} \mathrm{~K}_{2} \mathrm{CO}_{3}(14 \mathrm{mg}, 0.10 \mathrm{mmol})$. The reactants were suspended in THF (2 $\mathrm{mL})$. The vial was capped under ambient atmosphere, transferred to a Rayonet chamber photoreactor, and irradiated $(\mathrm{hv}=350 \mathrm{~nm})$ at $65{ }^{\circ} \mathrm{C}$. After $6 \mathrm{~h}$, the reaction mixture was cooled to rt and concentrated under reduced pressure. The resulting residue was purified by flash chromatography (neutralized $\mathrm{SiO}_{2}, 4: 1$ hexane/EtOAc) to afford $5 \mathrm{~b}$ (40 mg, $0.18 \mathrm{mmol}, 88 \%$ yield) as a colorless oil: ${ }^{1} \mathbf{H}$ NMR $\left(400 \mathrm{MHz} \mathrm{CDCl}_{3}\right) \delta$ $7.43(\mathrm{dd}, J=6.4,1.4 \mathrm{~Hz}, 1 \mathrm{H}), 7.01(\mathrm{td}, J=7.6,1.5 \mathrm{~Hz}, 1 \mathrm{H}), 6.79(\mathrm{td}, J=7.5,1.1 \mathrm{~Hz}$, $1 \mathrm{H}), 6.74(\mathrm{~d}, J=8.0,1 \mathrm{H}), 6.51(\mathrm{~d}, J=3.3,1 \mathrm{H}), 5.03(\mathrm{~d}, J=3.3,1 \mathrm{H}), 4.23(\mathrm{br} \mathrm{s}, 2 \mathrm{H})$, 3.36-3.33 (m, 4H), 2.00-1.97 (m, 4H); ${ }^{13} \mathbf{C}$ NMR (150 MHz, $\left.\mathrm{CDCl}_{3}\right) \delta$ C: 157.4, 143.0, 141.9, 117.7; CH: 126.7, 126.0, 118.7, 116.6, 109.4, 81.8; $\mathbf{C H}_{2}: 48.2,25.3$; IR (thin-film) 3253, 2970, $1518 \mathrm{~cm}^{-1}$; HRMS-ESI $(\mathrm{m} / \mathrm{z})[\mathrm{M}+\mathrm{H}]^{+}$calculated for $\mathrm{C}_{14} \mathrm{H}_{17} \mathrm{~N}_{2} \mathrm{O}=229.1335$; found 229.1335 .

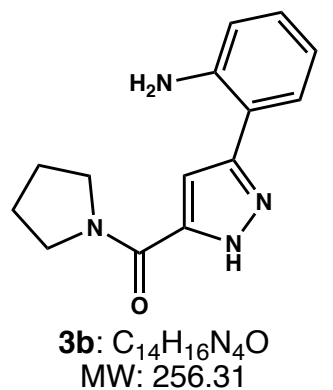

(3-(2-aminophenyl)-1H-pyrazol-5-yl)(pyrrolidin-1-yl)methanone (3b). $\mathbf{m p}=200$ $202{ }^{\circ} \mathrm{C} ;{ }^{1} \mathrm{H}$ NMR $\left(400 \mathrm{MHz}, \mathrm{CDCl}_{3}\right) \delta 7.49(\mathrm{~d}, J=7.6 \mathrm{~Hz}, 1 \mathrm{H}), 7.13(\mathrm{t}, J=7.1 \mathrm{~Hz}$, $1 \mathrm{H}), 6.87(\mathrm{~s}, 1 \mathrm{H}), 6.78-6.74(\mathrm{~m}, 2 \mathrm{H}), 3.85-3.83(\mathrm{~m}, 2 \mathrm{H}), 3.72-3.70(\mathrm{~m}, 2 \mathrm{H}), 2.11-$ $2.07(\mathrm{~m}, 2 \mathrm{H}), 2.00-1.95(\mathrm{~m}, 2 \mathrm{H}) ;{ }^{13} \mathrm{C}$ NMR $\left(150 \mathrm{MHz}, \mathrm{CDCl}_{3}\right) \delta \mathrm{C}: 158.3,152.7$, 145.1, 137.3, 115.8; $\mathbf{C H : ~ 1 2 9 . 0 , ~ 1 2 8 . 1 , ~ 1 1 7 . 3 , ~ 1 1 6 . 6 , ~ 1 0 4 . 0 ; ~} \mathrm{CH}_{2}: 48.0,47.1,26.5$, 23.9; IR (thin-film) 3320, 2973, $1593 \mathrm{~cm}^{-1}$; HRMS-ESI $(\mathrm{m} / \mathrm{z})[\mathrm{M}+\mathrm{H}]^{+}$calculated for $\mathrm{C}_{14} \mathrm{H}_{17} \mathrm{~N}_{4} \mathrm{O}=257.1397 ;$ found 257.1402 .

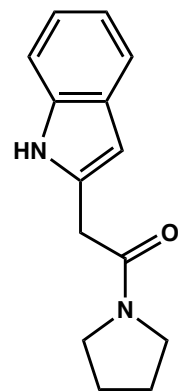

6: $\mathrm{C}_{14} \mathrm{H}_{16} \mathrm{~N}_{2} \mathrm{O}$ $M W: 228.30$

2-(1H-indol-2-yl)-1-(pyrrolidin-1-yl)ethan-1-one (6, Table 1, entry 8). A 1-dram vial was charged with pyridazine $N$-oxide $2 \mathbf{b}(26 \mathrm{mg}, 0.10 \mathrm{mmol})$. A solution of $\mathrm{Rh}_{2} \mathrm{esp}_{2}$ in THF (1 mol \%, $1 \mathrm{~mL}, 0.1 \mathrm{M}$ ) was added via syringe. The vial was capped, transferred to a Rayonet chamber photoreactor, and irradiated $(\mathrm{hv}=350 \mathrm{~nm})$ at $65{ }^{\circ} \mathrm{C}$. After $6 \mathrm{~h}$, the reaction mixture was cooled to $\mathrm{rt}$ and concentrated under reduced pressure. The resulting residue was purified by flash chromatography $\left(\mathrm{SiO}_{2}, 1: 2\right.$ hexane/EtOAc) to afford 6 (21 mg, $0.092 \mathrm{mmol}, 92 \%$ yield) as a yellow solid: $\mathrm{mp}=150-152{ }^{\circ} \mathrm{C} ;{ }^{1} \mathbf{H}$ NMR $\left(400 \mathrm{MHz} \mathrm{CDCl}_{3}\right) \delta 9.33(\mathrm{br} \mathrm{s}, 1 \mathrm{H}), 7.53(\mathrm{~d}, J=7.5 \mathrm{~Hz}, 1 \mathrm{H}), 7.33(\mathrm{~d}, J=8.2 \mathrm{~Hz}, 1 \mathrm{H})$, $7.13(\mathrm{t}, J=8.2 \mathrm{~Hz}, 1 \mathrm{H}), 7.06(\mathrm{t}, J=7.3 \mathrm{~Hz}, 1 \mathrm{H}), 6.29(\mathrm{~s}, 1 \mathrm{H}), 3.82(\mathrm{~s}, 2 \mathrm{H}), 3.55$ (t, $J=7.0$ $\mathrm{Hz}, 2 \mathrm{H}$ ), 3.49 (t, $J=6.9 \mathrm{~Hz}, 2 \mathrm{H}$ ), 1.95 (quint, $J=6.7 \mathrm{~Hz}, 2 \mathrm{H}$ ), 1.86 (quint, $J=6.7 \mathrm{~Hz}$, $2 \mathrm{H}) ;{ }^{13} \mathrm{C}$ NMR $\left(150 \mathrm{MHz}, \mathrm{CDCl}_{3}\right) \delta \mathrm{C}: 168.5,136.4,131.9,128.2 ; \mathrm{CH}: 121.4,119.8,119.6,111.0$, 100.8; $\mathbf{C H}_{2}$ : 47.1, 46.0, 34.4, 26.1, 24.4; IR (thin-film) 3259, 2981, $1638 \mathrm{~cm}^{-1}$; HRMS-DART (m/z) $[\mathrm{M}+\mathrm{H}]^{+}$calculated for $\mathrm{C}_{14} \mathrm{H}_{17} \mathrm{~N}_{2} \mathrm{O}=229.1335$; found 229.1339 . 
Single-operation synthesis of dihydrobiphenyl $(X=H, 7-12)$, tetrahydrocarbazole $\left(X,=\mathrm{NH}_{2} 13-\right.$ 22), and tetrahydrodibenzofuran derivatives $(X=O H, 23-25)$ shown in Scheme 3.

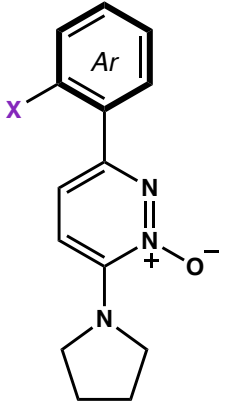

2

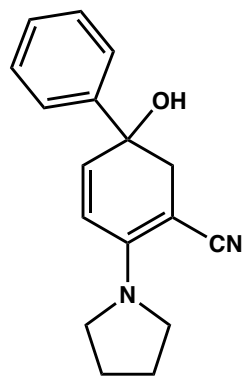

7: $\mathrm{C}_{17} \mathrm{H}_{18} \mathrm{~N}_{2} \mathrm{O}$ MW: 266.34 method A: hv= $350 \mathrm{~nm}$ $1.5 \mathrm{~mol} \% \mathrm{Rh}_{2} \mathrm{esp}_{2}$ $\operatorname{THF}(0.3 \mathrm{M}), 35^{\circ} \mathrm{C}$

method $\boldsymbol{B}: \mathrm{h} v=350 \mathrm{~nm}$ $3 \mathrm{~mol} \% \mathrm{Rh}_{2} \mathrm{esp}_{2}$, 0.5 equiv $\mathrm{K}_{2} \mathrm{CO}_{3}$

$\operatorname{THF}(0.1 \mathrm{M}), 65^{\circ} \mathrm{C}$

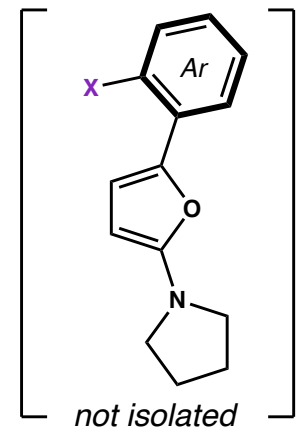

not isolated

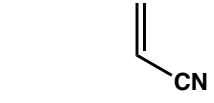

(1.5 to 5 equiv)

$\longrightarrow$ warm to $80^{\circ} \mathrm{C}$

removed from UV-light
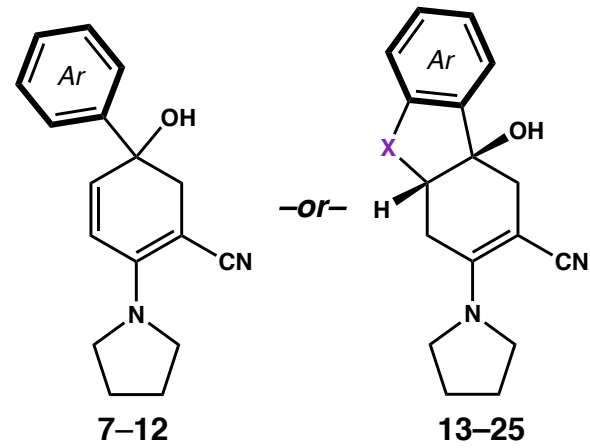

General procedure for the synthesis of 1-hydroxy-dihydrobiphenyl derivatives (Method A). Preparation of ( \pm )-1-hydroxy-4-(pyrrolidin-1-yl)-1,2-dihydro-[1,1'-biphenyl]3-carbonitrile (7). A 1-dram via was charged with pyridazine $\mathrm{N}$-oxide 2a (34 mg, 0.15 $\mathrm{mmol}$ ) and $\mathrm{Rh}_{2} \mathrm{esp}_{2}(1.5 \mathrm{mg}, 0.002 \mathrm{mmol}, 1.5 \mathrm{~mol} \%)$. The reactants were suspended in THF $(0.5 \mathrm{~mL})$ and the vial was capped under ambient atmosphere. ${ }^{9}$ The reaction vial was placed in a Rayonet chamber photoreactor and exposed to UV-light ( $h v=350$ $\mathrm{nm}$ ) at $35^{\circ} \mathrm{C}$. After $3 \mathrm{~h}$, the reaction mixture was removed from UV-light, cooled to rt, and treated with freshly distilled acrylonitrile $(0.2 \mathrm{~mL}, 0.3 \mathrm{mmol})$. The resulting suspension was then heated to $80{ }^{\circ} \mathrm{C}$ in a pre-warmed aluminum block. After $8 \mathrm{~h}$, the reaction was cooled to $\mathrm{rt}$ and concentrated under reduced pressure. The resulting residue was purified by flash chromatography (neutralized $\mathrm{SiO}_{2},{ }^{10} 3: 2$ hexane/EtOAc) to afford 7 (31 mg, $0.12 \mathrm{mmol}, 82 \%$ yield) as a yellow oil: ${ }^{1} \mathrm{H}$ NMR (400 MHz, $\left.\mathrm{CDCl}_{3}\right) \delta 7.49(\mathrm{~d}, \mathrm{~J}=7.1 \mathrm{~Hz}, 2 \mathrm{H}), 7.36$ $(\mathrm{t}, J=7.6 \mathrm{~Hz}, 2 \mathrm{H}), 7.30(\mathrm{t}, J=7.1 \mathrm{~Hz}, 1 \mathrm{H}), 6.36(\mathrm{~d}, J=10.1 \mathrm{~Hz}, 1 \mathrm{H}), 6.31(\mathrm{~d}, J=10.1 \mathrm{~Hz}, 1 \mathrm{H}), 3.68$ $(\mathrm{m}, 4 \mathrm{H}), 2.93(\mathrm{~d}, J=16.3 \mathrm{~Hz}, 1 \mathrm{H}), 2.78(\mathrm{~d}, J=16.3 \mathrm{~Hz}, 1 \mathrm{H}), 2.49(\mathrm{~s}, 1 \mathrm{H}), 1.94-1.93(\mathrm{~d}, J=6.8 \mathrm{~Hz}$, $4 \mathrm{H}) ;{ }^{13} \mathrm{C}$ NMR $\left(150 \mathrm{MHz}, \mathrm{CDCl}_{3}\right) \delta \mathrm{C}: 148.7,145.0,124.5,70.5,64.8 ; \mathbf{C H}: 140.0,128.3,127.7$, 125.2, 123.0; $\mathbf{C H}_{2}$ : 50.0, 42.4, 25.4; IR (thin-film) 3367, 2162, $1349 \mathrm{~cm}^{-1}$; HRMS-ESI $(\mathrm{m} / \mathrm{z})[\mathrm{M}+\mathrm{H}]^{+}$ calculated for $\mathrm{C}_{17} \mathrm{H}_{19} \mathrm{~N}_{2} \mathrm{O}=267.1497$; found 267.1506.

The following compounds were prepared in identical fashion from the corresponding derivatives of pyridazine $\mathrm{N}$-oxide $\mathbf{2 a}$.

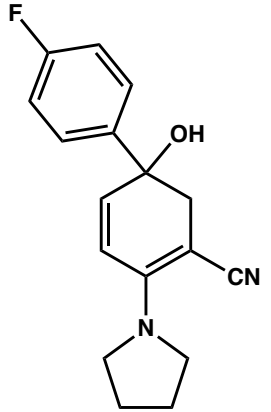

8: $\mathrm{C}_{17} \mathrm{H}_{17} \mathrm{FN}_{2} \mathrm{O}$ MW: 284.33

( \pm )-4'-fluoro-1-hydroxy-4-(pyrrolidin-1-yl)-1,2-dihydro-[1,1'-biphenyl]-3-carbonitrile (8). Following method A described above, the title compound was prepared from $\mathbf{2} \mathbf{a}_{\mathbf{1}}$ (39 $\mathrm{mg}, 0.15 \mathrm{mmol})$. The reaction mixture was irradiated at $35{ }^{\circ} \mathrm{C}$ for $6 \mathrm{~h}$. Following addition of acrylonitrile (1.5 equiv), the resulting solution was heated to $80{ }^{\circ} \mathrm{C}$ for 16 h. Purification by flash chromatography (neutralized $\mathrm{SiO}_{2}, 3: 2$ hexane/EtOAc) provided 8 (30 $\mathrm{mg}, 0.11 \mathrm{mmol}, 77 \%$ yield) as a yellow foam: ${ }^{1} \mathbf{H}$ NMR $(400 \mathrm{MHz}$, $\left.\mathrm{CDCl}_{3}\right) \delta 7.48-7.45(\mathrm{~m}, 2 \mathrm{H}), 7.03(\mathrm{t}, \mathrm{J}=8.7 \mathrm{~Hz}, 2 \mathrm{H}), 6.31(\mathrm{~s}, 2 \mathrm{H}), 3.67(\mathrm{~m}, 4 \mathrm{H}), 2.88$ $(\mathrm{d}, J=16.3 \mathrm{~Hz}, 1 \mathrm{H}), 2.76(\mathrm{~d}, J=16.3 \mathrm{~Hz}, 1 \mathrm{H}), 2.51(\mathrm{br} \mathrm{s}, 1 \mathrm{H}), 1.97-1.90(\mathrm{~m}, 4 \mathrm{H}) ;{ }^{13} \mathrm{C}$ NMR $\left(150 \mathrm{MHz}, \mathrm{CDCl}_{3}\right) \delta \mathrm{C}: 162.3(\mathrm{~d}, J=247 \mathrm{~Hz}), 148.6,140.7(\mathrm{~d}, J=3 \mathrm{~Hz}), 124.3$, 70.2, 64.8; CH: 139.9, 127.0 (d, $J=8 \mathrm{~Hz}$ ), 123.1, 115.2 (d, J = $21 \mathrm{~Hz}$ ); $\mathbf{C H}_{2}: 49.8$, 42.5, 25.4; IR (thin-film) 3364, 2970, $2166 \mathrm{~cm}^{-1}$; HRMS-ESI $(\mathrm{m} / \mathrm{z})[\mathrm{M}+\mathrm{H}]^{+}$calculated for $\mathrm{C}_{17} \mathrm{H}_{18} \mathrm{FNO}=285.1398$; found 285.1397. 


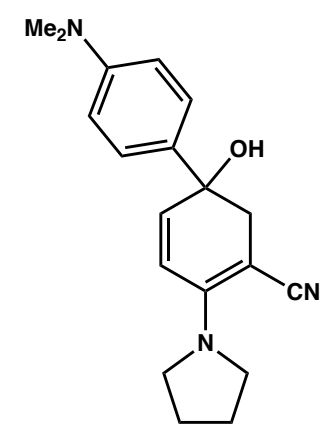

9: $\mathrm{C}_{19} \mathrm{H}_{23} \mathrm{~N}_{3} \mathrm{O}$ MW: 309.41

( \pm )-4'-(dimethylamino)-1-hydroxy-4-(pyrrolidin-1-yl)-1,2-dihydro-[1,1'-biphenyl]-3carbonitrile (9). Following method A described above, the title compound was prepared from $2 \mathbf{a}_{2}(43 \mathrm{mg}, 0.15 \mathrm{mmol})$. The reaction mixture was irradiated at 35 ${ }^{\circ} \mathrm{C}$ for $7 \mathrm{~h}$. Following addition of acrylonitrile (1.5 equiv), the resulting solution was heated to $80{ }^{\circ} \mathrm{C}$ for $10 \mathrm{~h}$. Purification by flash chromatography (neutralized $\mathrm{SiO}_{2}$, $4: 1 \rightarrow 1: 1$ hexane/EtOAc) provided 9 (24 mg, $0.08 \mathrm{mmol}, 52 \%$ yield) as a yellow oil: ${ }^{1} \mathrm{H}$ NMR (400 MHz, $\left.\mathrm{CDCl}_{3}\right) \delta 7.37-7.34(\mathrm{~m}, 2 \mathrm{H}), 6.73-6.70(\mathrm{~m}, 2 \mathrm{H}), 6.38(\mathrm{~d}, \mathrm{~J}=$ $10.1 \mathrm{~Hz}, 1 \mathrm{H}), 6.23(\mathrm{~d}, J=10.1 \mathrm{~Hz}, 1 \mathrm{H}), 3.67-3.64(\mathrm{~m}, 4 \mathrm{H}), 2.95(\mathrm{~s}, 6 \mathrm{H}), 2.90(\mathrm{~d}, J$ $=16.1 \mathrm{~Hz}, 1 \mathrm{H}), 2.81(\mathrm{~d}, J=16.1 \mathrm{~Hz}, 1 \mathrm{H}), 2.29(\mathrm{br} \mathrm{s}, 1 \mathrm{H}), 1.95-1.88(\mathrm{~m}, 4 \mathrm{H}) ;{ }^{13} \mathrm{C}$ NMR $\left(150 \mathrm{MHz}, \mathrm{CDCl}_{3}\right) \delta \mathrm{C}: 150.1,148.9,132.5,70.5,65.3 ; \mathbf{C H}: 141.0,126.0$, 124.7, 122.1, 112.2; $\mathbf{C H}_{2}: 49.7,40.5,25.4 ; \mathbf{C H}_{3}: 41.8$ IR (thin-film) 3400, 2871, $2164 \mathrm{~cm}^{-1}$; HRMS-ESI $(\mathrm{m} / \mathrm{z})[\mathrm{M}+\mathrm{H}]^{+}$calculated for $\mathrm{C}_{19} \mathrm{H}_{24} \mathrm{~N}_{3} \mathrm{O}=310.1914$; found 310.1928 .

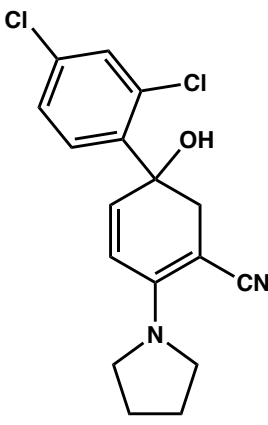

10: $\mathrm{C}_{17} \mathrm{H}_{16} \mathrm{Cl}_{2} \mathrm{~N}_{2} \mathrm{O}$ MW: 335.23

( \pm )-2',4'-dichloro-1-hydroxy-4-(pyrrolidin-1-yl)-1,2-dihydro-[1,1'-biphenyl]-3carbonitrile (10). Following method $A$ described above, the title compound was prepared from $2 \mathbf{a}_{3}(47 \mathrm{mg}, 0.15 \mathrm{mmol})$. The reaction mixture was irradiated at $35^{\circ} \mathrm{C}$ for $6 \mathrm{~h}$. Following addition of acrylonitrile (1.5 equiv), the resulting solution was heated to $80{ }^{\circ} \mathrm{C}$ for $8 \mathrm{~h}$. Purification by flash chromatography (neutralized $\mathrm{SiO}_{2}, 4: 1$ hexane/EtOAc) provided $10\left(35 \mathrm{mg}, 0.10 \mathrm{mmol}, 70 \%\right.$ yield) as a yellow foam: ${ }^{1} \mathrm{H}$ NMR $\left(400 \mathrm{MHz} \mathrm{CDCl}_{3}\right) \delta 7.81(\mathrm{~d}, J=8.5 \mathrm{~Hz}, 1 \mathrm{H}), 7.37(\mathrm{~s}, 1 \mathrm{H}), 7.29(\mathrm{~d}, J=8.4 \mathrm{~Hz}$, $1 \mathrm{H}), 6.38(\mathrm{~d}, J=9.9 \mathrm{~Hz}, 1 \mathrm{H}), 6.28(\mathrm{~d}, J=10.1 \mathrm{~Hz}, 1 \mathrm{H}), 3.70-3.68(\mathrm{~m}, 4 \mathrm{H}), 3.34(\mathrm{~d}, J$ $=16.8 \mathrm{~Hz}, 1 \mathrm{H}), 2.80(\mathrm{~s}, 1 \mathrm{H}), 2.55(\mathrm{~d}, J=16.5 \mathrm{~Hz}, 1 \mathrm{H}), 1.97-1.92(\mathrm{~m}, 4 \mathrm{H}) ;{ }^{13} \mathrm{C}$ NMR $\left(150 \mathrm{MHz} \mathrm{CDCl}_{3}\right) \delta \mathrm{C}: 148.5,140.1,134.2,131.8,124.3,69.8,64.7 ; \mathrm{CH}: 139.0$, 130.6, 129.0, 127.2, 121.8; $\mathbf{C H}_{2}: 49.8,39.2$, 25.4; IR (thin-film) 3353, 2968, $2166 \mathrm{~cm}^{-}$ 1; HRMS-ESI (m/z) [M+H] ${ }^{+}$calculated for $\mathrm{C}_{17} \mathrm{H}_{17} \mathrm{Cl}_{2} \mathrm{~N}_{2} \mathrm{O}=335.0712$; found 335.0725.

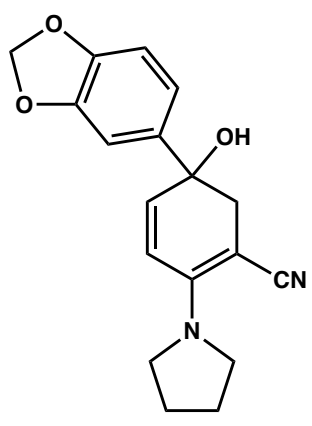

11: $\mathrm{C}_{18} \mathrm{H}_{18} \mathrm{~N}_{2} \mathrm{O}_{3}$ MW: 310.35

( \pm )-5-(benzo[d][1,3]dioxol-5-yl)-5-hydroxy-2-(pyrrolidine-1-yl)cyclohexa-1,3-diene1-carbonitrile (11). Following method A described above, the title compound was prepared from $2 \mathrm{a}_{4}(43 \mathrm{mg}, 0.15 \mathrm{mmol})$. The reaction mixture was irradiated at 35 ${ }^{\circ} \mathrm{C}$ for $4 \mathrm{~h}$. Following addition of acrylonitrile (1.5 equiv), the resulting solution was heated to $80{ }^{\circ} \mathrm{C}$ for $4 \mathrm{~h}$. Purification by flash chromatography (neutralized $\mathrm{SiO}_{2}, 3: 2$ hexane/EtOAc) provided $11\left(28 \mathrm{mg}, 0.09 \mathrm{mmol}, 60 \%\right.$ yield) as a yellow foam: ${ }^{1} \mathrm{H}$ NMR $\left(400 \mathrm{MHz} \mathrm{CDCl}_{3}\right) \delta 6.99(\mathrm{~d}, J=1.7 \mathrm{~Hz}, 1 \mathrm{H}), 6.93(\mathrm{dd}, J=6.4,1.8 \mathrm{~Hz}, 1 \mathrm{H})$, $6.77(\mathrm{~d}, J=8.1 \mathrm{~Hz}, 1 \mathrm{H}), 6.29(\mathrm{q}, J=5.1 \mathrm{~Hz}, 2 \mathrm{H}), 5.95(\mathrm{~s}, 2 \mathrm{H}), 3.66(\mathrm{~m}, 4 \mathrm{H}), 2.85$ (d, $J=16.2 \mathrm{~Hz}, 1 \mathrm{H}), 2.75(\mathrm{~d}, J=16.2 \mathrm{~Hz}, 1 \mathrm{H}), 1.96-1.88(\mathrm{~m}, 4 \mathrm{H}), 1.83(\mathrm{br} \mathrm{s}, 1 \mathrm{H})$; ${ }^{13} \mathrm{C}$ NMR $\left(150 \mathrm{MHz} \mathrm{CDCl}_{3}\right) \delta \mathrm{C}: 148.7,147.6,147.0,139.1,124.5,70.5,64.9 ; \mathbf{C H}:$ 140.1, 122.8, 118.5, 107.9, 106.2; $\mathbf{C H}_{2}:$ 101.1, 49.7, 42.5, 25.4; IR (thin-film) 3350, 2874, $2167 \mathrm{~cm}^{-1}$; HRMS-ESI $(\mathrm{m} / \mathrm{z})[\mathrm{M}+\mathrm{H}]^{+}$calculated for $\mathrm{C}_{18} \mathrm{H}_{19} \mathrm{~N}_{2} \mathrm{O}_{3}=311.1390$; found 311.1388.

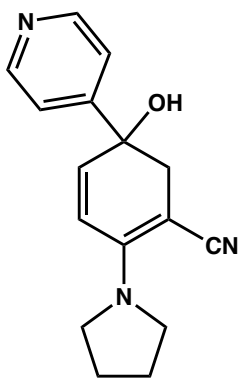

12: $\mathrm{C}_{16} \mathrm{H}_{17} \mathrm{~N}_{3} \mathrm{O}$ MW: 267.33
( \pm )-5-hydroxy-5-(pyridin-4-yl)-2-(pyrrolidin-1-yl)cyclohexa-1,3-diene-1-carbonitrile (12). Following method $A$ described above, the title compound was prepared from $2 \mathbf{a}_{5}$ (42 $\mathrm{mg}, 0.18 \mathrm{mmol}$ ). The reaction mixture was irradiated at $35{ }^{\circ} \mathrm{C}$ for $5 \mathrm{~h}$. Following addition of acrylonitrile (1.5 equiv), the resulting solution was heated to $80{ }^{\circ} \mathrm{C}$ for $16 \mathrm{~h}$. Purification by flash chromatography (neutralized $\mathrm{SiO}_{2}, 3: 2$ hexane/acetone) provided 12 (28 mg, $0.09 \mathrm{mmol}, 35 \%$ yield) as a yellow foam: ${ }^{1} \mathrm{H}$ NMR (400 MHz, CDCl $\mathrm{Cl}_{3} \delta 8.61$ (br s, 2H), $7.43(\mathrm{~d}, J=5.4 \mathrm{~Hz}, 2 \mathrm{H}), 6.38(\mathrm{~d}, J=10.1 \mathrm{~Hz}, 1 \mathrm{H}), 6.27(\mathrm{~d}, J=10.1 \mathrm{~Hz}, 1 \mathrm{H})$, 3.70-3.68 (m, 4H), $2.90(\mathrm{~d}, J=16.5 \mathrm{~Hz}, 1 \mathrm{H}), 2.75(\mathrm{~d}, J=16.4 \mathrm{~Hz}, 1 \mathrm{H}), 2.00-1.90(\mathrm{~m}$, $5 \mathrm{H}) ;{ }^{13} \mathrm{C}$ NMR $\left(150 \mathrm{MHz}, \mathrm{CDCl}_{3}\right) \delta \mathrm{C}: 153.9,148.5,124.0,69.8,64.5 ; \mathbf{C H}: 149.8$, 138.3, 123.9, 120.4; $\mathbf{C H}_{2}: 49.8,42.1,25.4$; IR (thin-film) 3300, 2969, $2165 \mathrm{~cm}^{-1}$; HRMS-ESI $(\mathrm{m} / \mathrm{z})[\mathrm{M}+\mathrm{H}]^{+}$calculated for $\mathrm{C}_{16} \mathrm{H}_{18} \mathrm{~N}_{3} \mathrm{O}=268.1444$; found 268.1443. 


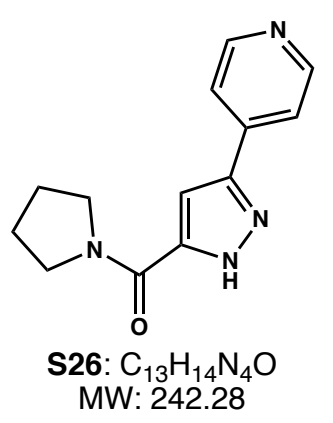

Pyrazole S26 (19 mg, $0.08 \mathrm{mmol}, 43 \%$ yield) was also isolated as a red solid: ${ }^{1} \mathbf{H}$ NMR $\left(400 \mathrm{MHz}, \mathrm{CDCl}_{3}\right) \delta 11.99(\mathrm{br} \mathrm{s}, 1 \mathrm{H}), 8.66(\mathrm{~d}, J=3.8 \mathrm{~Hz}, 2 \mathrm{H}), 7.73(\mathrm{~d}, J=$ $5.1 \mathrm{~Hz}, 1 \mathrm{H}), 6.96(\mathrm{~s}, 1 \mathrm{H}), 3.85(\mathrm{t}, J=6.8 \mathrm{~Hz}, 2 \mathrm{H}), 3.74(\mathrm{t}, J=6.8 \mathrm{~Hz}, 2 \mathrm{H}), 2.10$ (quint, $J=6.6 \mathrm{~Hz}, 2 \mathrm{H}$ ), 1.99 (quint, $J=6.8 \mathrm{~Hz}, 2 \mathrm{H}$ ); These and other characterization data were identical to those previously reported. ${ }^{6}$

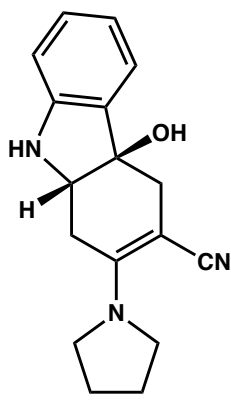

13: $\mathrm{C}_{17} \mathrm{H}_{19} \mathrm{~N}_{3} \mathrm{O}$ MW: 281.36

General procedure for the synthesis of 1-hydroxy-tetrahydrocarbazole and 1hydroxytetrahydrodibenzofuran derivatives (Method B). Preparation of ( \pm )-cis-4ahydroxy-2-(pyrrolidin-1-yl)-4,4a,9,9a-tetrahydro-1 H-carbazole-3-carbonitrile (13). A 1dram via was charged with pyridazine $\mathrm{N}$-oxide $\mathbf{2 b}(26 \mathrm{mg}, 0.10 \mathrm{mmol}), \mathrm{Rh}_{2} \mathrm{esp}_{2}(2 \mathrm{mg}$, $0.003 \mathrm{mmol}, 3 \mathrm{~mol} \%$ ), and powdered $\mathrm{K}_{2} \mathrm{CO}_{3}(7 \mathrm{mg}, 0.05 \mathrm{mmol})$. The reactants were suspended in THF $(1 \mathrm{~mL})$ and the vial was capped under ambient atmosphere. The reaction vial was placed in a Rayonet chamber photoreactor and exposed to UV-light $(h v=350 \mathrm{~nm})$ at $65^{\circ} \mathrm{C}$. After $6 \mathrm{~h}$, the reaction mixture was removed from UV-light, cooled to $\mathrm{rt}$, and treated with freshly distilled acrylonitrile $(0.3 \mathrm{~mL}, 0.5 \mathrm{mmol})$. The resulting slurry was then heated to $80^{\circ} \mathrm{C}$ in a pre-warmed aluminum block. After $9 \mathrm{~h}$, the reaction was cooled to $\mathrm{rt}$ and concentrated under reduced pressure. The resulting residue was purified by flash chromatography (neutralized $\mathrm{SiO}_{2}, 7: 3$ hexane/acetone) to afford 13 (21 $\mathrm{mg}, 0.08$ mmol, $75 \%$ yield) as a yellow foam: ${ }^{1} \mathrm{H}$ NMR $\left(400 \mathrm{MHz}, \mathrm{CDCl}_{3}\right) \delta 7.27(\mathrm{~d}, J=8.0 \mathrm{~Hz}, 1 \mathrm{H}), 7.13(\mathrm{t}, \mathrm{J}=$ $6.8 \mathrm{~Hz}, 1 \mathrm{H}), 6.80(\mathrm{t}, J=7.4 \mathrm{~Hz}, 1 \mathrm{H}), 6.59(\mathrm{~d}, J=7.9 \mathrm{~Hz}, 1 \mathrm{H}), 3.94(\mathrm{t}, J=5.2 \mathrm{~Hz}, 1 \mathrm{H}), 3.59(\mathrm{~m}, 4 \mathrm{H})$, 2.71-2.66 (m, 3H), $2.49(\mathrm{dd}, J=9.4,5.6 \mathrm{~Hz}, 1 \mathrm{H}), 1.91-1.87(\mathrm{~m}, 4 \mathrm{H}) ;{ }^{13} \mathrm{C}$ NMR $\left(150 \mathrm{MHz}, \mathrm{CDCl}_{3}\right) \delta$ C: $155.6,150.6,132.0,124.6,82.0,65.0 ; \mathbf{C H}: 129.9,123.7,119.6,110.0,66.3 ; \mathbf{C H}_{2}: 49.9,39.2$, 34.6, 25.3; IR (thin-film) 3335, 2163, $1567 \mathrm{~cm}^{-1}$; HRMS-ESI $(\mathrm{m} / \mathrm{z})[\mathrm{M}+\mathrm{H}]^{+}$calculated for $\mathrm{C}_{17} \mathrm{H}_{20} \mathrm{~N}_{3} \mathrm{O}=$ 282.1609; found 282.1599.

The following compounds were prepared in identical fashion from the corresponding variants of pyridazine $\mathrm{N}$-oxides $\mathbf{2} \mathbf{b}$ and $\mathbf{2 c}$.

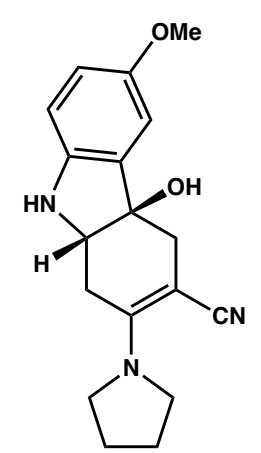

14: $\mathrm{C}_{18} \mathrm{H}_{21} \mathrm{~N}_{3} \mathrm{O}_{2}$ $\mathrm{MW}: 311.39$

( \pm )-cis-4a-hydroxy-6-methoxy-2-(pyrrolidin-1-yl)-4,4a,9,9a-tetrahydro-1H-carbazole-3carbonitrile (14). Following method $\mathrm{B}$ described above, the title compound was prepared from $2 \mathbf{b}_{1}(29 \mathrm{mg}, 0.10 \mathrm{mmol})$. The reaction mixture was irradiated at $65^{\circ} \mathrm{C}$ for $12 \mathrm{~h}$. Following addition of acrylonitrile (5 equiv), the resulting slurry was heated to 80 ${ }^{\circ} \mathrm{C}$ for $8 \mathrm{~h}$. Purification by flash chromatography (neutralized $\mathrm{SiO}_{2}, 100 \%$ hexanes $\rightarrow 100 \%$ EtOAc) provided $14(27 \mathrm{mg}, 0.09 \mathrm{mmol}, 87 \%$ yield $)$ as a yellow oil: ${ }^{1} \mathrm{H}$ NMR $\left(400 \mathrm{MHz}, \mathrm{CDCl}_{3}\right) \delta 6.86(\mathrm{~d}, J=2.6 \mathrm{~Hz}, 1 \mathrm{H}), 6.74(\mathrm{dd}, J=8.7,2.6 \mathrm{~Hz}, 1 \mathrm{H}), 6.56$ $(\mathrm{d}, J=8.6 \mathrm{~Hz}, 1 \mathrm{H}), 3.93(\mathrm{t}, J=5.5 \mathrm{~Hz}, 1 \mathrm{H}), 3.77(\mathrm{~s}, 3 \mathrm{H}), 3.63-3.59(\mathrm{~m}, 4 \mathrm{H}), 2.71-2.66$ (m, 3H), $2.48(\mathrm{dd}, J=15.0,5.8 \mathrm{~Hz}, 1 \mathrm{H}), 2.31(\mathrm{br} \mathrm{s}, 1 \mathrm{H}), 1.91-1.88(\mathrm{~m}, 4 \mathrm{H}) ;{ }^{13} \mathrm{C}$ NMR $\left(150 \mathrm{MHz}, \mathrm{CDCl}_{3}\right) \delta$ C: $155.6,154.3,144.3,133.2,124.5,82.4,64.9 ; \mathrm{CH}: 116.5,111.5$, 108.8, 67.0; $\mathbf{C H}_{2}$ : 49.9, 39.2, 34.7, 25.3; IR (thin-film) 3332, 2950, $2165 \mathrm{~cm}^{-1}$; HRMSDART $(\mathrm{m} / \mathrm{z})[\mathrm{M}+\mathrm{H}]^{+}$calculated for $\mathrm{C}_{18} \mathrm{H}_{22} \mathrm{~N}_{3} \mathrm{O}_{2}=312.1707$; found $=312.1711$. 


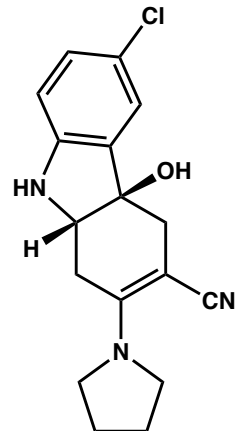

15: $\mathrm{C}_{17} \mathrm{H}_{18} \mathrm{CIN}_{3} \mathrm{O}$ MW: 315.80

( \pm )-cis-6-chloro-4a-hydroxy-2-(pyrrolidin-1-yl)-4,4a,9,9a-tetrahydro-1H-carbazole-3carbonitrile (15). Following method $\mathrm{B}$ described above, the title compound was prepared from $2 \mathbf{b}_{\mathbf{2}}(29 \mathrm{mg}, 0.10 \mathrm{mmol})$. The reaction mixture was irradiated at $65{ }^{\circ} \mathrm{C}$ for $5.5 \mathrm{~h}$. Following addition of acrylonitrile ( 5 equiv), the resulting slurry was heated to $80{ }^{\circ} \mathrm{C}$ for $16 \mathrm{~h}$. Purification by flash chromatography (neutralized $\mathrm{SiO}_{2}, 1: 1$ hexanes/EtOAc) provided 15 (19 mg, $0.06 \mathrm{mmol}, 59 \%$ yield) as a yellow solid: $\mathbf{m p}=$

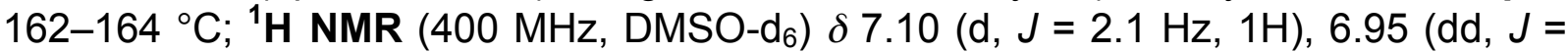
8.3, $2.4 \mathrm{~Hz}, 1 \mathrm{H}), 6.39(\mathrm{~d}, J=8.4 \mathrm{~Hz}, 1 \mathrm{H}), 5.90(\mathrm{~d}, J=3.0 \mathrm{~Hz}, 1 \mathrm{H}), 5.60(\mathrm{br} \mathrm{s}, 1 \mathrm{H})$, 3.81-3.78 (m, 1H), $3.46(\mathrm{br} \mathrm{s}, 1 \mathrm{H}), 2.60-2.41(\mathrm{~m}, 4 \mathrm{H}), 1.81(\mathrm{~m}, 4 \mathrm{H}) ;{ }^{13} \mathrm{C}$ NMR $(150$ $\left.\mathrm{MHz}, \mathrm{DMSO}-\mathrm{d}_{6}\right) \delta \mathrm{C}: 157.0,151.1,136.1,124.8,119.9,80.6,64.3 ; \mathrm{CH}: 128.7,123.8$, 109.5, 66.2; $\mathbf{C H}_{2}$ : 49.9, 40.5, 34.0, 25.3; IR (thin-film) 3296, 2978, $2170 \mathrm{~cm}^{-1}$; HRMSESI $(\mathrm{m} / \mathrm{z})[\mathrm{M}+\mathrm{Na}]^{+}$calculated for $\mathrm{C}_{17} \mathrm{H}_{18} \mathrm{ClN}_{3} \mathrm{ONa}=338.1031$; found $=338.1027$.

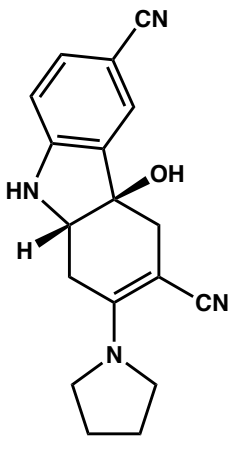

16: $\mathrm{C}_{18} \mathrm{H}_{18} \mathrm{~N}_{4} \mathrm{O}$ MW: 306.37

( \pm )-cis-4a-hydroxy-2-(pyrrolidin-1-yl)-4,4a,9,9a-tetrahydro-1H-carbazole-3,6 dicarbonitrile (16). Following a modification of method $B$ described above, the title compound was prepared from $\mathbf{2 b}_{3}(28 \mathrm{mg}, 0.10 \mathrm{mmol})$. The reaction mixture was irradiated at $65{ }^{\circ} \mathrm{C}$ for $3 \mathrm{~h}$. Following addition of acrylonitrile (5 equiv), the resulting slurry was heated to $80{ }^{\circ} \mathrm{C}$ for $9 \mathrm{~h}$. The reaction mixture was passed through a $\mathrm{SiO}_{2}$ plug with (100\% hexanes $\rightarrow 100 \%$ EtOAc). The mixture was concentrated under reduced pressure and then dissolved in THF $(1 \mathrm{~mL})$. The resulting solution was heated at $80{ }^{\circ} \mathrm{C}$. After $12 \mathrm{~h}$, the reaction mixture was cooled to it and concentrated under reduced pressure. Purification by flash chromatography (neutralized $\mathrm{SiO}_{2}, 7: 3$ hexane/acetone) provided 16 (22 mg, $0.072 \mathrm{mmol}, 72 \%$ yield) as a colorless foam: ${ }^{1} \mathrm{H}$ NMR $\left(400 \mathrm{MHz}\right.$, acetone- $\left.\mathrm{d}_{6}\right) \delta 7.51(\mathrm{~d}, J=1.4 \mathrm{~Hz}, 1 \mathrm{H}), 7.33(\mathrm{dd}, J=8.3,1.6 \mathrm{~Hz}, 1 \mathrm{H})$, $6.52(\mathrm{~d}, J=8.3 \mathrm{~Hz}, 1 \mathrm{H}), 6.13(\mathrm{br} \mathrm{s}, 1 \mathrm{H}), 4.78(\mathrm{~s}, 1 \mathrm{H}), 4.09-4.06(\mathrm{~m}, 1 \mathrm{H}), 3.58-3.52(\mathrm{~m}, 4 \mathrm{H}), 2.78(\mathrm{~d}$, $J=4.0 \mathrm{~Hz}, 1 \mathrm{H}), 2.69(\mathrm{~d}, J=4.1 \mathrm{~Hz}, 1 \mathrm{H}), 2.65(\mathrm{~d}, J=2.0 \mathrm{~Hz}, 1 \mathrm{H}), 1.88-1.85(\mathrm{~m}, 4 \mathrm{H}) ;{ }^{13} \mathrm{C}$ NMR $(150$ $\mathrm{MHz}$, acetone- $\left.\mathrm{d}_{6}\right) \delta \mathrm{C}: 155.7,155.3,134.2,123.2,120.2,98.2,80.2,65.3 ; \mathrm{CH}: 134.0,127.5,107.7$, 66.5; $\mathbf{C H}_{2}$ : 49.5, 39.7, 33.2, 25.0; IR (thin-film) 3275, 2202, $2174 \mathrm{~cm}^{-1}$; HRMS-ESI (m/z) $[\mathrm{M}+\mathrm{H}]^{+}$ calculated for $\mathrm{C}_{18} \mathrm{H}_{19} \mathrm{~N}_{4} \mathrm{O}=307.1553$; found 307.1562.

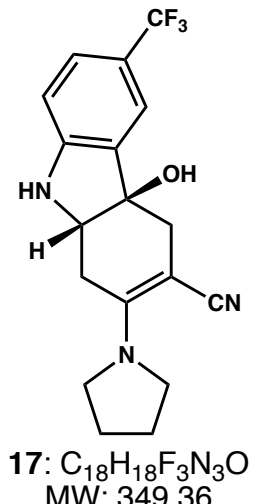

MW: 349.36

( \pm )-cis-4a-hydroxy-2-(pyrrolidin-1-yl)-6-(trifluoromethyl)-4,4a,9,9a-tetrahydro-1Hcarbazole-3-carbonitrile (17). Following method B described above, the title compound was prepared from $\mathbf{2} \mathbf{b}_{\mathbf{4}}(32 \mathrm{mg}, 0.10 \mathrm{mmol})$. The reaction mixture was irradiated at $65{ }^{\circ} \mathrm{C}$ for $1.5 \mathrm{~h}$. Following addition of acrylonitrile (5 equiv), the resulting slurry was heated to $80{ }^{\circ} \mathrm{C}$ for $16 \mathrm{~h}$. Purification by flash chromatography (neutralized $\mathrm{SiO}_{2}, 1: 1$ hexanes/EtOAc) provided $17(21 \mathrm{mg}, 0.06 \mathrm{mmol}, 60 \%$ yield) as a light brown solid: $\mathbf{m p}=80-83^{\circ} \mathrm{C} ;{ }^{1} \mathrm{H}$ NMR $\left(600 \mathrm{MHz}, \mathrm{CD}_{3} \mathrm{OD}\right) \delta 7.43(\mathrm{~s}, 1 \mathrm{H}), 7.29(\mathrm{~d}, J=8.3 \mathrm{~Hz}$, $1 \mathrm{H}), 6.53(\mathrm{~d}, J=8.3 \mathrm{~Hz}, 1 \mathrm{H}), 3.98(\mathrm{t}, J=4.1 \mathrm{~Hz}, 1 \mathrm{H}), 3.61-3.54(\mathrm{~m}, 4 \mathrm{H}), 2.78(\mathrm{dd}, J=$ 15.2, 3.7 Hz, 1H), 2.68-2.61 (m, 3H), $2.00(\mathrm{br} \mathrm{s}, 1 \mathrm{H}), 1.92-1.88(\mathrm{~m}, 4 \mathrm{H}) ;{ }^{13} \mathrm{C}$ NMR $\left(150 \mathrm{MHz}, \mathrm{CDCl}_{3}\right) \delta \mathrm{C}: 155.2,153.2,131.9,125.6,124.0,121.4,81.4,64.9 ; \mathbf{C H}:$ $127.7(\mathrm{~d}, J=3 \mathrm{~Hz}), 121.2$ (d, $J=4 \mathrm{~Hz}), 108.9,66.8 ; \mathbf{C H}_{2}: 49.9,39.3,34.2,25.3$; IR (thin-film) 3306, 2921, $2158 \mathrm{~cm}^{-1}$; HRMS-ESI $(\mathrm{m} / \mathrm{z})[\mathrm{M}+\mathrm{Na}]^{+}$calculated for $\mathrm{C}_{18} \mathrm{H}_{18} \mathrm{~F}_{3} \mathrm{~N}_{3} \mathrm{ONa}=$ 372.1294 ; found $=372.1309$. 


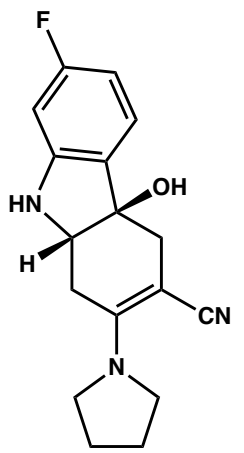

18: $\mathrm{C}_{17} \mathrm{H}_{18} \mathrm{FN}_{3} \mathrm{O}$ MW: 299.35

( \pm )-cis-7-fluoro-4a-hydroxy-2-(pyrrolidin-1-yl)-4,4a,9,9a-tetrahydro-1H-carbazole-3carbonitrile (18). Following a modification of method $B$ described above, the title compound was prepared from $2 \mathbf{b}_{5}(27 \mathrm{mg}, 0.10 \mathrm{mmol})$. The reaction mixture was irradiated at $65{ }^{\circ} \mathrm{C}$ for $5 \mathrm{~h}$. Following addition of acrylonitrile (5 equiv), the resulting slurry was heated to $80{ }^{\circ} \mathrm{C}$ for $10 \mathrm{~h}$. The reaction mixture was passed through a $\mathrm{SiO}_{2}$ plug with $(100 \%$ hexanes $\rightarrow 100 \%$ EtOAc). The mixture was concentrated under reduced pressure and then dissolved in THF $(1 \mathrm{~mL})$. The resulting solution was heated at $80{ }^{\circ} \mathrm{C}$. After $12 \mathrm{~h}$, the reaction mixture was cooled to $\mathrm{rt}$ and concentrated under reduced pressure. Purification by flash chromatography (neutralized $\mathrm{SiO}_{2}, 1: 3$ hexane/EtOAc) provided 18 (16 $\mathrm{mg}, 0.05 \mathrm{mmol}, 55 \%$ yield) as a yellow foam: ${ }^{1} \mathbf{H}$ NMR $\left(400 \mathrm{MHz} \mathrm{CDCl}_{3}\right) \delta 7.18(\mathrm{dd}, J=5.8,2.5,1 \mathrm{H}), 6.46(\mathrm{td}, J=9.4,2.2,1 \mathrm{H}), 6.25(\mathrm{dd}, J=$ 9.9, $2.3 \mathrm{~Hz}, 1 \mathrm{H}), 3.99$ (br s, 2H), 3.61-3.57 (m, 4H), 2.67-2.64 (m, 3H), 2.51 (dd, J = 9.8, 5.2 Hz, 1H), 2.30 (br s, $1 \mathrm{H}), 1.91-1.88(\mathrm{~m}, 4 \mathrm{H}) ;{ }^{13} \mathbf{C}$ NMR (150 MHz, acetone-d 6$) \delta$ C: $164.3(\mathrm{~d}, \mathrm{~J}=240 \mathrm{~Hz}), 155.9$, 153.6 (d, J = $12 \mathrm{~Hz}$ ), 129.3, 80.3, 66.9, 65.6; CH: 124.4 (d, J = $11 \mathrm{~Hz}), 103.1$ (d, J = $23 \mathrm{~Hz}), 95.3$ (d, J = $26 \mathrm{~Hz}$ ); $\mathbf{C H}_{2}$ : 49.5, 40.0, 33.7, 25.0; IR (thin-film) 3339, 2164, $1348 \mathrm{~cm}^{-1}$; HRMS-DART (m/z) $[\mathrm{M}+\mathrm{H}]^{+}$calculated for $\mathrm{C}_{17} \mathrm{H}_{19} \mathrm{FN} \mathrm{N}_{3} \mathrm{O}=300.1507$; found 300.1502 .

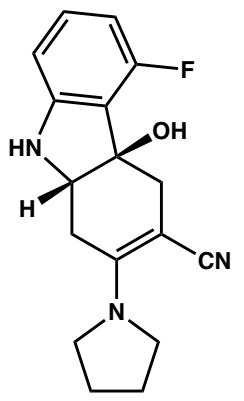

19: $\mathrm{C}_{17} \mathrm{H}_{18} \mathrm{FN}_{3} \mathrm{O}$ MW: 299.35

( \pm )-cis-5-fluoro-4a-hydroxy-2-(pyrrolidin-1-yl)-4,4a,9,9a-tetrahydro-1H-carbazole-3carbonitrile (19). Following a modification of method B described above, the title compound was prepared from $2 \mathbf{b}_{6}(27 \mathrm{mg}, 0.10 \mathrm{mmol})$. The reaction mixture was irradiated at $65{ }^{\circ} \mathrm{C}$ for $3 \mathrm{~h}$. Following addition of acrylonitrile (5 equiv), the resulting slurry was heated to $80{ }^{\circ} \mathrm{C}$ for $4 \mathrm{~h}$. The reaction mixture was passed through a $\mathrm{SiO}_{2}$ plug with (100 \% hexanes $\rightarrow 100 \%$ EtOAc). The mixture was concentrated under reduced pressure and then dissolved in THF $(1 \mathrm{~mL})$. The resulting solution was heated at $80{ }^{\circ} \mathrm{C}$. After $12 \mathrm{~h}$, the reaction mixture was cooled to $\mathrm{rt}$ and concentrated under reduced pressure. Purification by flash chromatography (neutralized $\mathrm{SiO}_{2}, 1: 1$ hexane/EtOAc) provided 19 (23 $\mathrm{mg}, 0.08 \mathrm{mmol}, 78 \%$ yield) as a yellow foam: ${ }^{1} \mathbf{H}$ NMR $\left(400 \mathrm{MHz}, \mathrm{DMSO}_{-} \mathrm{d}_{6}\right) \delta 6.96(\mathrm{td}, J=6.0,2.1 \mathrm{~Hz}, 1 \mathrm{H}), 6.23(\mathrm{dd}, J=7.8,3.2 \mathrm{~Hz}, 2 \mathrm{H}), 6.06(\mathrm{br} \mathrm{s}, 1 \mathrm{H})$, 5.64 (br s, 1H), 3.79-3.77 (m, 1H), $3.47(\mathrm{~m}, 4 \mathrm{H}), 2.67(\mathrm{~d}, J=14.2 \mathrm{~Hz}, 1 \mathrm{H}), 2.54-2.52(\mathrm{~m}, 3 \mathrm{H}), 1.81$ $(\mathrm{m}, 4 \mathrm{H}) ;{ }^{13} \mathrm{C}$ NMR $\left(150 \mathrm{MHz}, \mathrm{CDCl}_{3}\right) \delta \mathrm{C}: 160.2(\mathrm{~d}, \mathrm{~J}=245 \mathrm{~Hz}), 156.8,154.9(\mathrm{~d}, \mathrm{~J}=9 \mathrm{~Hz}), 124.7$, 118.3 (d, $J=18$ Hz, 80.6, 64.6; CH: 131.1 (d, $J=9 \mathrm{~Hz}), 104.7,103.6$ (d, J = $21 \mathrm{~Hz}$ ), 66.6; CH 38.7, 33.9, 25.3; IR (thin-film) 3364, 2871, $2166 \mathrm{~cm}^{-1}$; HRMS-ESI (m/z) [M+H] ${ }^{+}$calculated for $\mathrm{C}_{17} \mathrm{H}_{19} \mathrm{FN}_{3} \mathrm{O}=300.1507$; found 300.1510 .

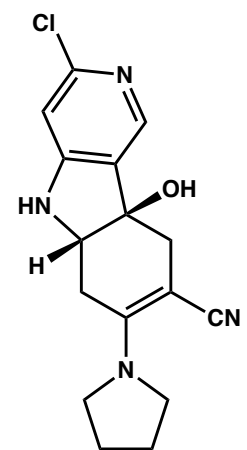

20: $\mathrm{C}_{16} \mathrm{H}_{17} \mathrm{CIN}_{4} \mathrm{O}$ MW: 316.79

( \pm )-cis-3-chloro-9a-hydroxy-7-(pyrrolidin-1-yl)-5a,6,9,9a-tetrahydro-5H-pyrido[4,3b]indole-8-carbonitrile (20). Following method B described above, the title compound was prepared from $\mathbf{2} \mathbf{b}_{7}(29 \mathrm{mg}, 0.10 \mathrm{mmol})$. The reaction mixture was irradiated at 65 ${ }^{\circ} \mathrm{C}$ for $6 \mathrm{~h}$. Following addition of acrylonitrile (5 equiv), the resulting slurry was heated to $80{ }^{\circ} \mathrm{C}$ for $4 \mathrm{~h}$. Purification by flash chromatography (neutralized $\mathrm{SiO}_{2}, 1: 3$ hexanes/EtOAc) provided 20 (16 mg, $0.06 \mathrm{mmol}, 60 \%$ yield) as a brown foam: ${ }^{1} \mathbf{H}$ NMR (400 MHz, acetone- $\left.\mathrm{d}_{6}\right) \delta 7.96(\mathrm{~s}, 1 \mathrm{H}), 6.35(\mathrm{~s}, 1 \mathrm{H}), 6.32(\mathrm{br} \mathrm{s}, 1 \mathrm{H}), 4.88(\mathrm{br} \mathrm{s}, 1 \mathrm{H})$, 4.10-4.08 (m, 1H), 3.57-3.53 (m, 4H), 2.84-2.79 (m, 2H), 1.89-1.86 (m, 4H); ${ }^{13} \mathrm{C}$ NMR (150 MHz, acetone- $\left.\mathrm{d}_{6}\right) \delta \mathbf{C}: 159.5,155.6,151.2,129.6,123.3,79.1,65.2 ; \mathbf{C H}$ : 143.4, 101.5, 67.3; $\mathbf{C H}_{2}$ : 49.5, 39.7, 33.0, 25.0; IR (thin-film) 3299, 2166, $1348 \mathrm{~cm}^{-1}$; HRMS-ESI $(\mathrm{m} / \mathrm{z})[\mathrm{M}+\mathrm{H}]^{+}$calculated for $\mathrm{C}_{16} \mathrm{H}_{18} \mathrm{CIN}_{4} \mathrm{O}=317.1164$; found 317.1159. 


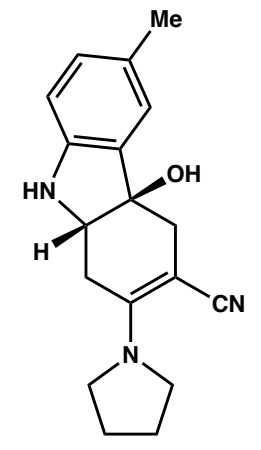

21: $\mathrm{C}_{18} \mathrm{H}_{21} \mathrm{~N}_{3} \mathrm{O}$ MW: 295.39

( \pm )-cis-4a-hydroxy-6-methyl-2-(pyrrolidin-1-yl)-4,4a,9,9a-tetrahydro-1H-carbazole-3carbonitrile (21). Following method B described above, the title compound was prepared from $2 \mathbf{b}_{\mathbf{8}}(27 \mathrm{mg}, 0.10 \mathrm{mmol})$. The reaction mixture was irradiated at $65{ }^{\circ} \mathrm{C}$ for $6 \mathrm{~h}$. Following addition of acrylonitrile (5 equiv), the resulting slurry was heated to $80^{\circ} \mathrm{C}$ for $9 \mathrm{~h}$. Purification by flash chromatography (neutralized $\mathrm{SiO}_{2}, 1: 1$ hexanes/EtOAc) provided 21 (23 mg, $0.078 \mathrm{mmol}, 78 \%$ yield) as a yellow oil: ${ }^{1} \mathbf{H}$ NMR (400 $\left.\mathrm{MHz}, \mathrm{CDCl}_{3}\right)$ $\delta 7.07(\mathrm{br} \mathrm{s}, 1 \mathrm{H}), 6.94(\mathrm{~d}, J=7.6 \mathrm{~Hz}, 1 \mathrm{H}), 6.51(\mathrm{~d}, J=7.8 \mathrm{~Hz}, 1 \mathrm{H}), 3.91(\mathrm{t}, J=5.3 \mathrm{~Hz}$, $1 \mathrm{H}), 3.61-3.58(\mathrm{~m}, 4 \mathrm{H}), 2.71-2.66(\mathrm{~m}, 3 \mathrm{H}), 2.46(\mathrm{dd}, J=9.2,5.6 \mathrm{~Hz}, 1 \mathrm{H}), 2.27(\mathrm{~s}, 3 \mathrm{H})$,

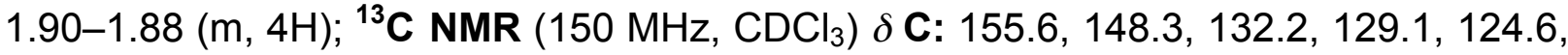
82.1, 65.0; CH: 130.5, 124.0, 110.2, 66.7; $\mathbf{C H}_{2}: 49.9,39.2,34.7,25.3 ; \mathbf{C H}_{3}: 20.8$; IR (thin-film) 3319, 2921, $2164 \mathrm{~cm}^{-1}$; HRMS-ESI $(\mathrm{m} / \mathrm{z})[\mathrm{M}+\mathrm{H}]^{+}$calculated for $\mathrm{C}_{18} \mathrm{H}_{22} \mathrm{~N}_{3} \mathrm{O}=$ 296.1757; found 296.1770.

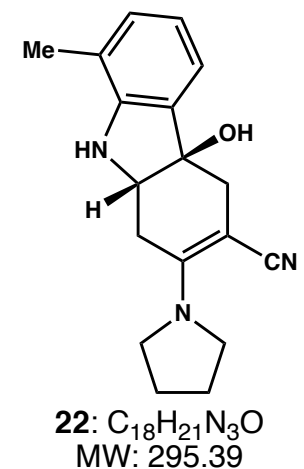

( \pm )-cis-4a-hydroxy-8-methyl-2-(pyrrolidin-1-yl)-4,4a,9,9a-tetrahydro-1H-carbazole-3carbonitrile (22). Following method B described above, the title compound was prepared from $2 \mathbf{b}_{9}(27 \mathrm{mg}, 0.10 \mathrm{mmol})$. The reaction mixture was irradiated at $65{ }^{\circ} \mathrm{C}$ for $6 \mathrm{~h}$. Following addition of acrylonitrile ( 5 equiv), the resulting slurry was heated to $80{ }^{\circ} \mathrm{C}$ for $2 \mathrm{~h}$. Purification by flash chromatography (neutralized $\mathrm{SiO}_{2}, 1: 1$ hexanes/EtOAc) provided $22\left(22 \mathrm{mg}, 0.077 \mathrm{mmol}, 77 \%\right.$ yield) as a yellow foam: ${ }^{1} \mathrm{H}$ NMR $\left(400 \mathrm{MHz} \mathrm{CDCl}_{3}\right) \delta 7.12(\mathrm{~d}, J=6.9 \mathrm{~Hz}, 1 \mathrm{H}), 6.96(\mathrm{~d}, J=6.9 \mathrm{~Hz}, 1 \mathrm{H}), 6.74(\mathrm{t}, J$ $=7.1 \mathrm{~Hz}, 1 \mathrm{H}), 3.91(\mathrm{t}, J=5.4 \mathrm{~Hz}, 1 \mathrm{H}), 3.76(\mathrm{br} \mathrm{s}, 1 \mathrm{H}), 3.59(\mathrm{~m}, 4 \mathrm{H}), 2.74-2.64(\mathrm{~m}$, $3 \mathrm{H}), 2.50$ (br s, 1H), $2.46(\mathrm{dd}, J=8.9,6.1 \mathrm{~Hz}, 1 \mathrm{H}), 2.09(\mathrm{~s}, 3 \mathrm{H}), 1.90-1.86(\mathrm{~m}, 4 \mathrm{H})$;

${ }^{13} \mathbf{C}$ NMR $\left(150 \mathrm{MHz}, \mathrm{CDCl}_{3}\right) \delta$ C: $155.6,149.1,131.3,124.6,119.4,82.4,65.3 ; \mathbf{C H}:$ 130.5, 121.0, 119.8, 66.3; $\mathbf{C H}_{2}: 49.9,39.2,34.8,25.4 ; \mathbf{C H}_{3}: 16.6$; IR (thin-film) 3322, 2967, $2154 \mathrm{~cm}^{-}$ 1; HRMS-ESI $(\mathrm{m} / \mathrm{z})[\mathrm{M}+\mathrm{H}]^{+}$calculated for $\mathrm{C}_{18} \mathrm{H}_{22} \mathrm{~N}_{3} \mathrm{O}=296.1757$; found 296.1753. Relative stereochemistry was confirmed by single crystal X-ray diffractions (CCDC entry 1895817). ${ }^{11}$

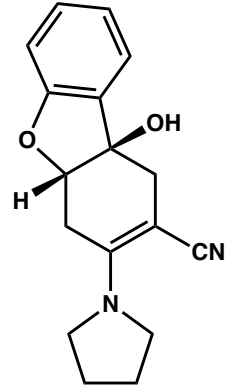

23: $\mathrm{C}_{17} \mathrm{H}_{18} \mathrm{~N}_{2} \mathrm{O}_{2}$ MW: 282.34

( \pm )-cis-9b-hydroxy-3-(pyrrolidin-1-yl)-1,4,4a,9b-tetrahydrodibenzo[b,d]furan-2carbonitrile (23). Following method B described above, the title compound was prepared from 2c $(39 \mathrm{mg}, 0.15 \mathrm{mmol})$. The reaction mixture was irradiated at $65^{\circ} \mathrm{C}$ for $7 \mathrm{~h}$. Following addition of acrylonitrile (5 equiv), the resulting slurry was heated to 80 ${ }^{\circ} \mathrm{C}$ for $7 \mathrm{~h}$. Purification by flash chromatography (neutralized $\mathrm{SiO}_{2}, 1: 1$ hexanes/EtOAc) provided 23 (26 mg, $0.092 \mathrm{mmol}, 61 \%$ yield) as a colorless oil: ${ }^{1} \mathbf{H}$ NMR (400 MHz, $\left.\mathrm{CDCl}_{3}\right) \delta 7.34(\mathrm{~d}, J=7.3 \mathrm{~Hz}, 1 \mathrm{H}), 7.23(\mathrm{t}, J=8.0 \mathrm{~Hz}, 1 \mathrm{H}), 6.96(\mathrm{t}, J=7.1 \mathrm{~Hz}, 1 \mathrm{H}), 6.74$ $(\mathrm{d}, J=7.6 \mathrm{~Hz}, 1 \mathrm{H}), 4.76(\mathrm{t}, J=4.2 \mathrm{~Hz}, 1 \mathrm{H}), 3.63-3.53(\mathrm{~m}, 4 \mathrm{H}), 2.89$ (dd, J = 15.4, 4.5 $\mathrm{Hz}, 1 \mathrm{H}), 2.78-2.67(\mathrm{~m}, 3 \mathrm{H}), 2.33(\mathrm{~s}, 1 \mathrm{H}), 1.90-1.87(\mathrm{~m}, 4 \mathrm{H}) ;{ }^{13} \mathrm{C}$ NMR $(150 \mathrm{MHz}$, $\left.\mathrm{CDCl}_{3}\right) \delta \mathrm{C}: 160.3,154.0,130.0,124.0,81.4,65.0 ; \mathbf{C H}: 130.6,123.6,121.6,109.8$, 88.8; $\mathbf{C H}_{2}$ : 49.9, 38.9, 33.1, 25.3; IR (thin-film) 3361, 2951, $2171 \mathrm{~cm}^{-1}$; HRMS-ESI $(\mathrm{m} / \mathrm{z})[\mathrm{M}+\mathrm{H}]^{+}$ calculated for $\mathrm{C}_{17} \mathrm{H}_{19} \mathrm{~N}_{2} \mathrm{O}_{2}=283.1441$; found 283.1441 . 


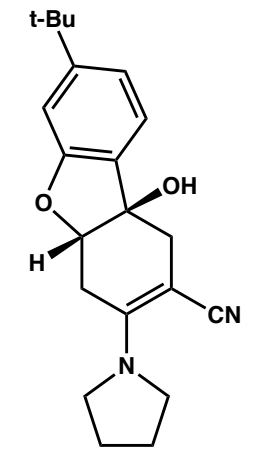

24: $\mathrm{C}_{21} \mathrm{H}_{26} \mathrm{~N}_{2} \mathrm{O}_{2}$ MW: 338.45

( \pm )-cis-9b-hydroxy-6-methoxy-8-methyl-3-(pyrrolidin-1-yl)-1,4,4a,9btetrahydrodibenzo[b,d]furan-2-carbonitrile (24). Following method B described above, the title compound was prepared from $2 \mathbf{c}_{1}(31 \mathrm{mg}, 0.10 \mathrm{mmol})$. The reaction mixture was irradiated at $65^{\circ} \mathrm{C}$ for $5 \mathrm{~h}$. Following addition of acrylonitrile ( 5 equiv), the resulting slurry was heated to $80{ }^{\circ} \mathrm{C}$ for $5 \mathrm{~h}$. Purification by flash chromatography (neutralized $\mathrm{SiO}_{2}, 1: 1$ hexanes/EtOAc) provided $24(22 \mathrm{mg}, 0.065 \mathrm{mmol}, 65 \%$ yield) as a yellow oil: ${ }^{1} \mathrm{H}$ NMR $\left(400 \mathrm{MHz}, \mathrm{CDCl}_{3}\right) \delta 7.24(\mathrm{~d}, J=8.0 \mathrm{~Hz}, 1 \mathrm{H}), 6.99(\mathrm{dd}, J=6.4,1.5 \mathrm{~Hz}, 1 \mathrm{H})$, $6.76(\mathrm{~d}, J=1.4 \mathrm{~Hz}, 1 \mathrm{H}), 4.72(\mathrm{t}, J=4.4 \mathrm{~Hz}, 1 \mathrm{H}), 3.65-3.53(\mathrm{~m}, 4 \mathrm{H}), 2.84$ (dd, $J=10.9$, $4.6 \mathrm{~Hz}, 1 \mathrm{H}), 2.76-2.67(\mathrm{~m}, 2 \mathrm{H}), 2.43(\mathrm{~s}, 1 \mathrm{H}), 1.90-1.88(\mathrm{~m}, 4 \mathrm{H}), 1.29(\mathrm{~s}, 9 \mathrm{H}) ;{ }^{13} \mathrm{C}$ NMR $\left(150 \mathrm{MHz} \mathrm{CDCl}_{3}\right) \delta \mathrm{C}: 160.4,154.6,154.1,127.0,124.2,81.1,65.2,35.0 ; \mathbf{C H}: 122.9$, 118.9, 106.9, 89.1; $\mathbf{C H}_{2}: 49.9,38.8,33.1,25.3 ; \mathbf{C H}_{3}: 31.4$; IR (thin-film) 3369, 2963. $2171 \mathrm{~cm}^{-1}$; HRMS-ESI $(\mathrm{m} / \mathrm{z})[\mathrm{M}+\mathrm{H}]^{+}$calculated for $\mathrm{C}_{21} \mathrm{H}_{27} \mathrm{~N}_{2} \mathrm{O}_{2}=339.2067$; found 339.2080.

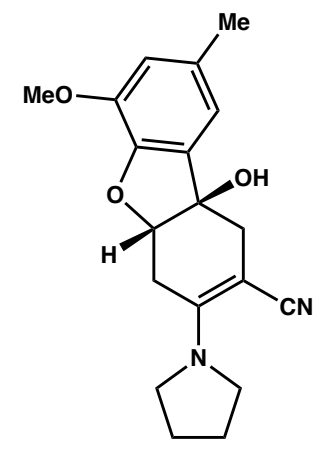

25: $\mathrm{C}_{19} \mathrm{H}_{22} \mathrm{~N}_{2} \mathrm{O}_{3}$ MW: 326.40

( \pm )-cis-9b-hydroxy-6-methoxy-8-methyl-3-(pyrrolidin-1-yl)-1,4,4a,9btetrahydrodibenzo[b,d]furan-2-carbonitrile (25). Following method B described above, the title compound was prepared from $2 \mathbf{c}_{2}(30 \mathrm{mg}, 0.10 \mathrm{mmol})$. The reaction mixture was irradiated at $65{ }^{\circ} \mathrm{C}$ for $5.5 \mathrm{~h}$. Following addition of acrylonitrile ( 5 equiv), the resulting slurry was heated to $80{ }^{\circ} \mathrm{C}$ for $7 \mathrm{~h}$. Purification by flash chromatography (neutralized $\mathrm{SiO}_{2}, 1: 2$ hexanes/EtOAc) provided $25(29 \mathrm{mg}, 0.088$ mmol, $88 \%$ yield) as a colorless oil: ${ }^{1} \mathrm{H}$ NMR (400 MHz, $\left.\mathrm{CDCl}_{3}\right) \delta 6.73(\mathrm{~s}, 1 \mathrm{H}), 6.62$ (s, 1H), 4.78 (t, J = 4.3 Hz, 1H), $3.85(\mathrm{~s}, 3 \mathrm{H}), 3.63-3.55(\mathrm{~m}, 4 \mathrm{H}), 2.90$ (dd, $J=15.7$ $\mathrm{Hz}, 4.5,1 \mathrm{H}), 2.76-2.66(\mathrm{~m}, 3 \mathrm{H}), 2.34$ (br s, 1H), $2.32(\mathrm{~s}, 3 \mathrm{H}), 1.89-1.86(\mathrm{~m}, 4 \mathrm{H})$; ${ }^{13} \mathrm{C}$ NMR $\left(150 \mathrm{MHz}_{\mathrm{CDCl}}\right) \delta \mathrm{C}: 154.1,146.6,143.8,132.1,130.8,124.2,81.9$, 64.9; $\mathbf{C H}: 115.3,114.0,89.5 ; \mathbf{C H}_{2}: 49.9,38.6,33.1,25.3 ; \mathbf{C H}_{3}: 55.9,21.3$; IR (thinfilm) 3326, 2926, $2172 \mathrm{~cm}^{-1}$; HRMS-ESI $(\mathrm{m} / \mathrm{z})[\mathrm{M}+\mathrm{H}]^{+}$calculated for $\mathrm{C}_{19} \mathrm{H}_{23} \mathrm{~N}_{2} \mathrm{O}_{3}=327.1703$; found 327.1718.

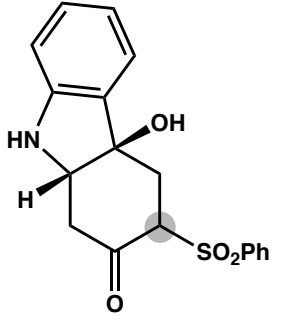

26: $\mathrm{C}_{18} \mathrm{H}_{17} \mathrm{NO}_{4} \mathrm{~S}$ MW: 343.40 $1: 1$ d.r.

( \pm )-cis-4a-hydroxy-3-(phenylsulfonyl)-1,3,4,4a,9,9a-hexahydro-2H-carbazol-2-one (26). A 1-dram vial was charged with $5 \mathrm{~b}(23 \mathrm{mg}, 0.10 \mathrm{mmol})$ and phenyl vinyl sulfone (50 $\mathrm{mg}, 0.3 \mathrm{mmol})$. The reactants were dissolved in THF $(1 \mathrm{~mL})$ and the vial was capped under ambient atmosphere. The reaction mixture was heated to $80{ }^{\circ} \mathrm{C}$ in a pre-warmed aluminum block. After $6 \mathrm{~h}$, the reaction was cooled to rt, treated with $\mathrm{SiO}_{2}(600 \mathrm{mg})$, and stirred for $1 \mathrm{~h}$. The resulting slurry was filtered and the filtrate was washed with $\mathrm{CH}_{2} \mathrm{Cl}_{2}(5 \mathrm{~mL})$. The combined organic extracts were then concentrated under reduced pressure. The resulting residue was purified by flash chromatography $\left(\mathrm{SiO}_{2}, 1: 1\right.$ hexane/EtOAc) to afford $26(23 \mathrm{mg}, 0.067 \mathrm{mmol}, 68 \%$ yield) as 1:1 mixture of diastereomers (inseparable on $\left.\mathrm{SiO}_{2}\right):{ }^{1} \mathbf{H} \mathbf{~ N M R}(400 \mathrm{MHz}$, $\left.\mathrm{CDCl}_{3}\right) D_{A} \delta 7.80(\mathrm{~d}, J=7.1 \mathrm{~Hz}, 1 \mathrm{H}), 6.57(\mathrm{~d}, J=8.2 \mathrm{~Hz}, 1 \mathrm{H}), 3.92(\mathrm{t}, J=7.6 \mathrm{~Hz}, 1 \mathrm{H}), 3.10(\mathrm{dd}, J=$ 15.0, $4.4 \mathrm{~Hz}, 1 \mathrm{H}), 2.51(\mathrm{dd}, J=15.0,2.8 \mathrm{~Hz}, 1 \mathrm{H}) ; \boldsymbol{D}_{B} \delta 7.86(\mathrm{~d}, J=7.1 \mathrm{~Hz}, 1 \mathrm{H}), 6.67(\mathrm{~d}, J=8.2 \mathrm{~Hz}$, $1 \mathrm{H}), 3.45(\mathrm{dd}, J=12.8,5.8 \mathrm{~Hz}, 1 \mathrm{H}), 2.73-2.68(\mathrm{~m}, 2 \mathrm{H})$; the remaining ${ }^{1} \mathrm{H}$ signals could not be resolved; ${ }^{13} \mathbf{C}$ NMR $\left(150 \mathrm{MHz}_{\mathrm{CDCl}}\right) \boldsymbol{D}_{\boldsymbol{A}} \delta \mathbf{C}: 199.4,149.3,137.7,78.7 ; \mathbf{C H}: 134.2,131.0,129.2$, 129.1, 123.6, 120.0, 66.8, 66.2; $\mathbf{C H}_{2}: 44.0,33.4 ; \boldsymbol{D}_{B} \delta \mathbf{C}: 199.8,148.8,137.8,78.3 ; \mathbf{C H}: 134.2,130.9$, 129.2, 129.1, 123.3, 119.9, 67.8, 67.1; $\mathbf{C H}_{2}: 44.6,32.7$; IR (thin-film) 3359, 2969, $1724 \mathrm{~cm}^{-1}$; HRMSESI $(m / z)[M+H]^{+}$calculated for $\mathrm{C}_{18} \mathrm{H}_{18} \mathrm{NO}_{4} \mathrm{~S}=344.0951$; found 344.0959. 


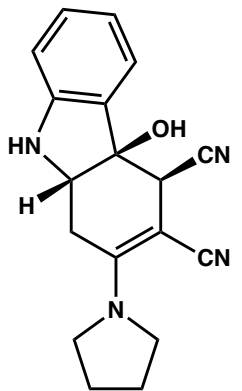

27: $\mathrm{C}_{18} \mathrm{H}_{18} \mathrm{~N}_{4} \mathrm{O}$ MW: 306.37

( \pm )-4a-hydroxy-2-(pyrrolidin-1-yl)-4,4a,9,9a-tetrahydro-1H-carbazole-3,4-dicarbonitrile (27). A 1-dram vial was charged with $\mathbf{5 b}(23 \mathrm{mg}, 0.10 \mathrm{mmol})$ and $(E)$-fumaronitrile (39 $\mathrm{mg}, 0.5 \mathrm{mmol})$. The reactants were dissolved in THF $(1 \mathrm{~mL})$ and the vial was capped under ambient atmosphere. The reaction mixture was heated to $80^{\circ} \mathrm{C}$ in a pre-warmed aluminum block. After $4 \mathrm{~h}$, the mixture was cooled to $\mathrm{rt}$ and concentrated under reduced pressure. Purification by flash chromatography (neutralized $\mathrm{SiO}_{2}, 1: 2$ hexane/EtOAc) provided 27 (17 mg, $0.056 \mathrm{mmol}, 56 \%$ yield) as a yellow foam: ${ }^{1} \mathrm{H}$ NMR $\left(400 \mathrm{MHz}, \mathrm{DMSO}-\mathrm{d}_{6}\right) \delta 7.27(\mathrm{~d}, J=7.4 \mathrm{~Hz}, 1 \mathrm{H}), 7.01(\mathrm{t}, J=7.3 \mathrm{~Hz}, 1 \mathrm{H}), 6.58(\mathrm{t}, J=7.3$ $\mathrm{Hz}, 1 \mathrm{H}), 6.43(\mathrm{~d}, J=7.8 \mathrm{~Hz}, 1 \mathrm{H}), 6.30(\mathrm{br} \mathrm{s}, 1 \mathrm{H}), 5.93(\mathrm{~d}, J=3.0 \mathrm{~Hz}, 1 \mathrm{H}), 3.97(\mathrm{~s}, 1 \mathrm{H})$, 3.91-3.89 (m, 1H), 3.53-3.48 (m, 4H), 2.82-2.81 (m, 2H), 1.84-1.81 (m, 4H); ${ }^{13} \mathrm{C}$ NMR $\left(150 \mathrm{MHz}, \mathrm{DMSO}-\mathrm{d}_{6}\right) \delta \mathrm{C}: 158.5,152.4,131.1,122.9,120.1,80.8,62.3 ; \mathrm{CH}: 130.0,124.1,117.3$, 108.7, 64.1; $\mathbf{C H}_{2}$ : 50.4, 34.2, 25.2; IR (thin-film) 3367, 2923, $2172 \mathrm{~cm}^{-1}$; HRMS-ESI (m/z) [M+Na] calculated for $\mathrm{C}_{18} \mathrm{H}_{18} \mathrm{~N}_{4} \mathrm{ONa}=329.1373$; found 329.1383 .

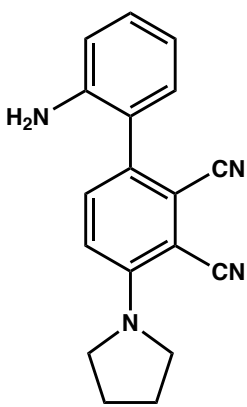

S27: $\mathrm{C}_{18} \mathrm{H}_{16} \mathrm{~N}_{4}$ MW: 288.35

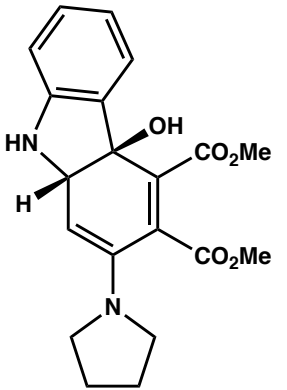

28: $\mathrm{C}_{20} \mathrm{H}_{22} \mathrm{~N}_{2} \mathrm{O}_{5}$ MW: 370.41

2'-amino-4-(pyrrolidin-1-yl)-[1,1'-biphenyl]-2,3-dicarbonitrile (S27). Isolated as a sideproduct in the reaction of $\mathbf{5 b}$ and $(E)$-fumaronitrile to give $\mathbf{2 7}$. Exposure of $\mathbf{2 7}$ to mild acid forms S26 in >95\% yield: $\mathbf{m p}=112-114{ }^{\circ} \mathrm{C} ;{ }^{1} \mathbf{H}$ NMR $\left(400 \mathrm{MHz}, \mathrm{CDCl}_{3}\right) \delta 7.43$ $(\mathrm{d}, J=9.2 \mathrm{~Hz} 1 \mathrm{H}), 7.21(\mathrm{t}, J=7.8 \mathrm{~Hz}, 1 \mathrm{H}), 7.08(\mathrm{~d}, J=7.4 \mathrm{~Hz}, 1 \mathrm{H}), 6.92(\mathrm{~d}, J=8.8$ $\mathrm{Hz}, 1 \mathrm{H}), 6.84(\mathrm{t}, J=7.4 \mathrm{~Hz}, 1 \mathrm{H}), 6.78(\mathrm{~d}, J=7.8 \mathrm{~Hz}, 1 \mathrm{H}), 3.69(\mathrm{~m}, 4 \mathrm{H}), 3.58(\mathrm{~s}, 2 \mathrm{H})$, $2.06(\mathrm{~m}, 4 \mathrm{H}) ;{ }^{13} \mathrm{C}$ NMR $\left(150 \mathrm{MHz}, \mathrm{CDCl}_{3}\right) \delta \mathrm{C}: 149.7,143.9,132.1,122.7,118.2$, 118.0, 116.0, 96.1; CH: 135.2, 130.7, 130.0, 119.1, 119.0, 116.1; $\mathbf{C H}_{2}: 50.3$, 25.8; IR (thin-film) 3462, 3369, $2208 \mathrm{~cm}^{-1}$; HRMS-ESI $(\mathrm{m} / \mathrm{z})[\mathrm{M}+\mathrm{H}]^{+}$calculated for $\mathrm{C}_{18} \mathrm{H}_{17} \mathrm{~N}_{4}=$ 289.1448; found 289.1459.

Dimethyl

cis-4a-hydroxy-2-(pyrrolidin-1-yl)-4a,9a-dihydro-9H-carbazole-3,4dicarboxylate (28). A 1-dram vial was charged with $5 \mathbf{b}(23 \mathrm{mg}, 0.10 \mathrm{mmol})$ and dimethyl acetylenedicarboxylate $(43 \mathrm{mg}, 0.30 \mathrm{mmol})$. The reactants were dissolved in THF (1 mL) and the vial was capped under ambient atmosphere. The reaction mixture was stirred to $80{ }^{\circ} \mathrm{C}$ in a pre-warmed aluminum block. After $1 \mathrm{~h}$, the mixture was cooled to rt and concentrated under reduced pressure. Purification by flash chromatography (neutralized $\mathrm{SiO}_{2}, 1: 1$ hexane/EtOAc) provided 28 (29 mg, 0.078 mmol, $78 \%$ yield) as a yellow oil: ${ }^{1} \mathbf{H}$ NMR $\left(400 \mathrm{MHz}, \mathrm{CDCl}_{3}\right) \delta 7.16(\mathrm{~d}, J=7.1 \mathrm{~Hz}$, $1 \mathrm{H}), 7.11(\mathrm{~d}, J=8.1 \mathrm{~Hz}, 1 \mathrm{H}), 6.74(\mathrm{t}, J=7.5 \mathrm{~Hz}, 1 \mathrm{H}), 6.70(\mathrm{~d}, J=7.6 \mathrm{~Hz}, 1 \mathrm{H}), 6.45$ $(\mathrm{d}, J=10.2,1 \mathrm{H}), 6.24(\mathrm{~d}, J=10.6,1 \mathrm{H}), 5.21(\mathrm{br} \mathrm{s}, 1 \mathrm{H}), 3.72(\mathrm{~s}, 3 \mathrm{H}), 3.67(\mathrm{~s}, 3 \mathrm{H})$, 3.60-3.54 (m, 2H), 3.16-3.13 (m, 2H), $2.64(\mathrm{br} \mathrm{s}, 1 \mathrm{H}), 1.97-1.93(\mathrm{~m}, 2 \mathrm{H}), 1.80-1.74(\mathrm{~m}, 2 \mathrm{H}) ;{ }^{13} \mathrm{C}$ NMR $\left(150 \mathrm{MHz}, \mathrm{CDCl}_{3}\right) \delta$ C: 173.5, 166.6, 150.0, 149.3, 130.7, 92.4, 79.0, 78.1; CH: 133.5, 129.9, 123.9, 121.8, 119.3, 110.2; $\mathbf{C H}_{2}: 51.8,25.4 ; \mathbf{C H}_{3}$ : 52.6, 50.6; IR (thin-film) 3390, 2952, $1729 \mathrm{~cm}^{-1}$; HRMS-ESI $(\mathrm{m} / \mathrm{z})[\mathrm{M}+\mathrm{H}]^{+}$calculated for $\mathrm{C}_{20} \mathrm{H}_{23} \mathrm{~N}_{2} \mathrm{O}_{5}=371.1601$; found 371.1610 .

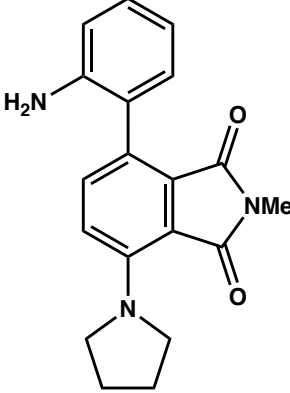

29: $\mathrm{C}_{19} \mathrm{H}_{19} \mathrm{~N}_{3} \mathrm{O}_{2}$ MW: 321.38 4-(2-aminophenyl)-2-methyl-7-(pyrrolidin-1-yl)isoindoline-1,3-dione (29). A 1-dram vial was charged with $\mathbf{5 b}(23 \mathrm{mg}, 0.10 \mathrm{mmol})$ and $\mathrm{N}$-methylmaleimide $(33 \mathrm{mg}, 0.3$ $\mathrm{mmol})$. The reactants were dissolved in THF $(1 \mathrm{~mL})$ and the vial was capped under ambient atmosphere. The reaction mixture was maintained at $23^{\circ} \mathrm{C}$. After $0.5 \mathrm{~h}$, the mixture was concentrated under reduced pressure. Purification by flash chromatography (neutralized $\mathrm{SiO}_{2}, 1: 2$ hexane/EtOAc) provided $29(27 \mathrm{mg}, 0.079$ mmol, $79 \%$ yield) as a yellow oil: $\mathbf{m p}=152-154{ }^{\circ} \mathrm{C} ;{ }^{1} \mathbf{H}$ NMR $\left(400 \mathrm{MHz}, \mathrm{CDCl}_{3}\right) \delta$ $7.36(\mathrm{~d}, J=8.8 \mathrm{~Hz}, 1 \mathrm{H}), 7.20(\mathrm{t}, J=7.4 \mathrm{~Hz}, 1 \mathrm{H}), 7.06(\mathrm{~d}, J=7.4 \mathrm{~Hz}, 1 \mathrm{H}), 7.01(\mathrm{~d}, J$ $=8.8 \mathrm{~Hz}, 1 \mathrm{H}), 6.84(\mathrm{t}, J=7.5 \mathrm{~Hz}, 1 \mathrm{H}), 6.79(\mathrm{~d}, J=8.0 \mathrm{~Hz}, 1 \mathrm{H}), 3.65-3.61(\mathrm{~m}, 5 \mathrm{H})$, 
3.05 (s, 3H), 2.05-2.00 (m, 4H); ${ }^{13} \mathbf{C}$ NMR $\left(150 \mathrm{MHz}, \mathrm{CDCl}_{3}\right) \delta$ C: $168.1,167.8,145.7,144.2,131.1$, 125.4, 123.9, 111.8; $\mathbf{C H}: 137.3,130.5,129.0,120.8,118.5,115.7 ; \mathbf{C H}_{2}: 51.8,25.9 ; \mathbf{C H}_{3}: 23.7$; IR (thin-film) 3426, 3383, $1692 \mathrm{~cm}^{-1}$; HRMS-ESI $(\mathrm{m} / \mathrm{z})[\mathrm{M}+\mathrm{H}]^{+}$calculated for $\mathrm{C}_{19} \mathrm{H}_{19} \mathrm{~N}_{3} \mathrm{O}_{2} \mathrm{Na}=344.1369$; found 344.1383 .

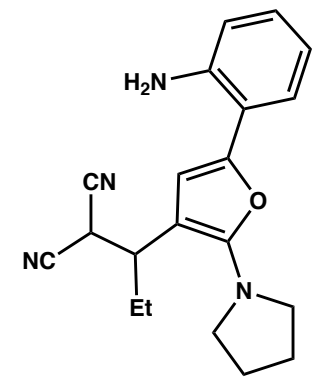

30: $\mathrm{C}_{20} \mathrm{H}_{22} \mathrm{~N}_{4} \mathrm{O}$ MW: 334.42

(土)-2-(1-(5-(2-aminophenyl)-2-(pyrrolidin-1-yl)furan-3-yl)propyl)malononitrile (30). A 1-dram vial was charged with $\mathbf{5 b}(40 \mathrm{mg}, 0.18 \mathrm{mmol})$ and 2-propylidenemalonotrile $(95 \mathrm{mg}, 0.9 \mathrm{mmol})$. The reactants were dissolved in THF $(1.8 \mathrm{~mL})$ and the vial was capped under ambient atmosphere. The reaction mixture was maintained at $23^{\circ} \mathrm{C}$. After $3 \mathrm{~h}$, the mixture was concentrated under reduced pressure. Purification by flash chromatography (neutralized $\mathrm{SiO}_{2}, 1: 1$ hexane/Et $\mathrm{t}_{2} \mathrm{O}$ ) provided $\mathbf{3 0}(40 \mathrm{mg}, 0.12$ mmol, $60 \%$ yield) as a brown oil: ${ }^{1} \mathrm{H}$ NMR $\left(400 \mathrm{MHz}, \mathrm{CDCl}_{3}\right) \delta 7.41(\mathrm{dd}, J=7.8,1.3$, $1 \mathrm{H}), 7.06(\mathrm{td}, J=7.7,1.5 \mathrm{~Hz}, 1 \mathrm{H}), 6.78(\mathrm{td}, J=7.6,1.0 \mathrm{~Hz}, 1 \mathrm{H}), 6.73(\mathrm{dd}, J=8.0$, $1.0 \mathrm{~Hz}, 1 \mathrm{H}), 6.50(\mathrm{~s}, 1 \mathrm{H}), 4.17(\mathrm{br} \mathrm{s}, 2 \mathrm{H}), 3.91(\mathrm{~d}, J=6.5 \mathrm{~Hz}, 1 \mathrm{H}), 3.44-3.39(\mathrm{~m}$, $2 \mathrm{H}), 3.37-3.28(\mathrm{~m}, 3 \mathrm{H}), 2.05-1.95(\mathrm{~m}, 5 \mathrm{H}), 1.89-1.78(\mathrm{~m}, 1 \mathrm{H}), 0.98(\mathrm{t}, J=7.3 \mathrm{~Hz}$, $3 \mathrm{H}) ;{ }^{13} \mathrm{C}$ NMR $\left(150 \mathrm{MHz}, \mathrm{CDCl}_{3}\right) \delta$ C: $153.9,145.4,142.5,116.4,112.5,112.4,100.7 ; \mathbf{C H}: 128.0$, 126.5, 118.7, 116.8, 106.2, 39.2, 29.7; $\mathbf{C H}_{2}: 50.7,25.8,25.3 ; \mathbf{C H}_{3}: 11.9$; IR (thin-film) 3324, 2970, $2180 \mathrm{~cm}^{-1}$; HRMS-DART $(\mathrm{m} / \mathrm{z})[\mathrm{M}+\mathrm{H}]^{+}$calculated for $\mathrm{C}_{20} \mathrm{H}_{23} \mathrm{~N}_{4} \mathrm{O}=335.1872$; found 335.1879.

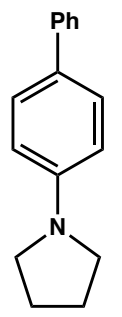

31: $\mathrm{C}_{16} \mathrm{H}_{17} \mathrm{~N}$ MW: 223.32

1-([1,1'-biphenyl]-4-yl)pyrrolidine (31). A 1-dram vial was charged with 5a (42 mg, 0.20 $\mathrm{mmol})$ and benzyl acrylate $(162 \mathrm{mg}, 1.00 \mathrm{mmol})$. The reactants were dissolved in THF $(2.0 \mathrm{~mL})$ and the vial was capped under ambient atmosphere. The reaction mixture was heated to $120^{\circ} \mathrm{C}$ in a pre-warmed aluminum block. After $38 \mathrm{~h}$, the mixture was cooled to it and concentrated under reduced pressure. Purification by flash chromatography (neutralized $\mathrm{SiO}_{2}$, hexanes) provided $31(22 \mathrm{mg}, 0.14 \mathrm{mmol}, 73 \%$ yield) as a colorless solid and benzyl alcohol (22 mg, $0.15 \mathrm{mmol}, 73 \%$ yield). Characterization data for $31:{ }^{1} \mathrm{H}$ $\operatorname{NMR}\left(400 \mathrm{MHz}, \mathrm{CDCl}_{3}\right) \delta 7.50(\mathrm{~d}, J=7.3 \mathrm{~Hz}, 2 \mathrm{H}), 7.30(\mathrm{t}, J=8.1 \mathrm{~Hz}, 2 \mathrm{H}), 7.09(\mathrm{t}, J=7.4$ $\mathrm{Hz}, 1 \mathrm{H}), 6.58(\mathrm{~d}, J=3.3 \mathrm{~Hz}, 1 \mathrm{H}), 5.01(\mathrm{~d}, J=3.3 \mathrm{~Hz}, 1 \mathrm{H}), 3.35(\mathrm{brt}, J=6.6 \mathrm{~Hz}, 4 \mathrm{H}$ ), 1.99 (quint, $J=$ $3.4 \mathrm{~Hz}, 4 \mathrm{H})$. These and other characterization data were identical to those previously reported. ${ }^{12}$

\section{Supplementary Optimization Tables}

General Procedure. A 1-dram vial was charged with 2a (26 mg, $0.1 \mathrm{mmol}), \mathrm{Rh}_{2} \mathrm{esp}_{2}(2 \mathrm{mg}, 0.003$ $\mathrm{mmol})$ and additive $(0.05 \mathrm{mmol}, 0.5$ equiv). The reactants were suspended in THF $(1 \mathrm{~mL})$ and the vial was sealed under ambient atmosphere. The rapidly stirred reaction slurry was irradiated at $65^{\circ} \mathrm{C}$ with $350 \mathrm{~nm}$ UV-light. After $3 \mathrm{~h}$, the reaction was removed from UV-light, cooled to rt, filtered, and concentrated under reduced pressure. The resulting residue was suspended in benzene $(1 \mathrm{~mL})$ and concentrated again. The unpurified reaction mixture was then analyzed by ${ }^{1} \mathrm{H}$ NMR spectroscopy $(400 \mathrm{MHz})$ to determine the relative ratio of products $\mathbf{5 b}, \mathbf{6}$, and $\mathbf{3 b}$. Notably, $\mathrm{K}_{2} \mathrm{CO}_{3}, \mathrm{KOH}$, and $\mathrm{K}_{3} \mathrm{PO}_{4}$ performed equally well in our experiments. Accordingly, $\mathrm{K}_{2} \mathrm{CO}_{3}$ was selected as the optimal additive because of its low cost and universal availability. 
Table S1. Preliminary additive screen for the conversion of $N$-oxide $\mathbf{2 b}$ to 2 -aminofuran $\mathbf{5 b}$.
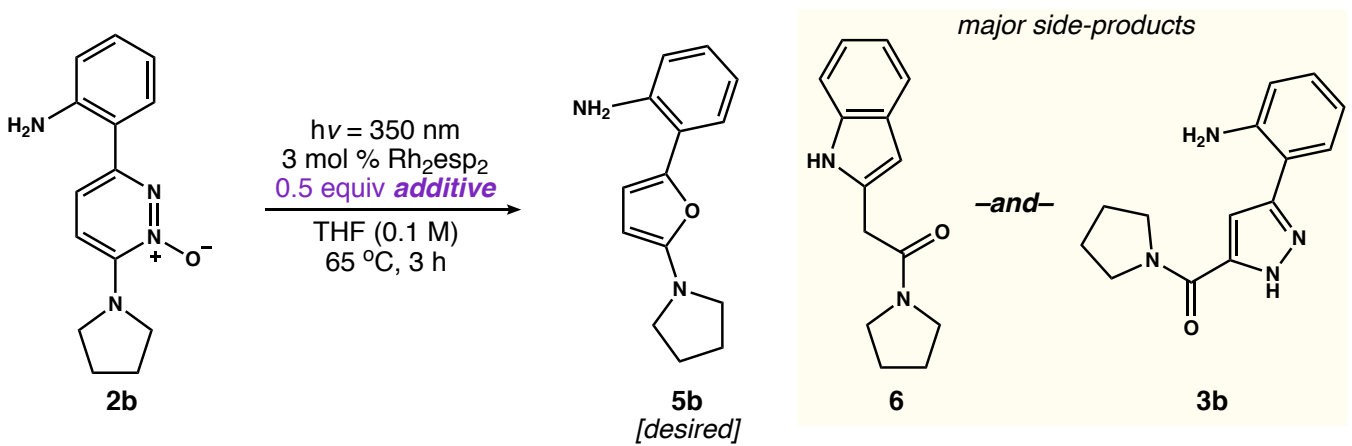

\begin{tabular}{ccc} 
entry & additive & ratio $\mathbf{5 b}: \mathbf{6}: \mathbf{3 b}$ \\
1 & none & $0: 1: 0$ \\
2 & $\mathrm{~K}_{2} \mathrm{CO}_{3}$ & $>20: 1: 0$ \\
3 & $2 \mathrm{M} \mathrm{aq.} \mathrm{K}_{2} \mathrm{CO}_{3}$ & $5: 1: 0$ \\
4 & $\mathrm{KOH}$ & $>20: 1: 0$ \\
5 & $\mathrm{~K}_{3} \mathrm{PO}_{4}$ & $>20: 1: 0$ \\
6 & $\mathrm{KHCO}_{3}$ & $3: 1: 0$ \\
7 & $\mathrm{KOAC}_{3}$ & $10: 1: 0$ \\
8 & $\mathrm{~K}_{2} \mathrm{SO}_{4}$ & $1: 18: 0$ \\
9 & $\mathrm{KCN}$ & $0: 20: 1$ \\
10 & $\mathrm{KBr}$ & $1: 20: 0$ \\
11 & $\mathrm{KF}$ & $5: 1: 0$ \\
12 & $\mathrm{Li}_{2} \mathrm{CO}_{3}$ & $1: 20: 0$ \\
13 & $\mathrm{Na}_{2} \mathrm{CO}_{3}$ & $12: 4: 1$ \\
14 & $\mathrm{Cs}_{2} \mathrm{CO}_{3}$ & $10: 0: 1$ \\
15 & $\mathrm{Et}_{3} \mathrm{~N}$ & $4: 1: 2$ \\
16 & $\mathrm{DBU}_{1}$ & $1: 0: 2$ \\
17 & $\mathrm{pyridine}$ & $0: 1: 1$ \\
\hline
\end{tabular}

Table S2. Catalyst loading optimization for photosubstrate $\mathbf{2 a}$.<smiles>Nc1ccccc1-c1ccc(N2C[CH+]CC2)[n+]([O-])n1</smiles>
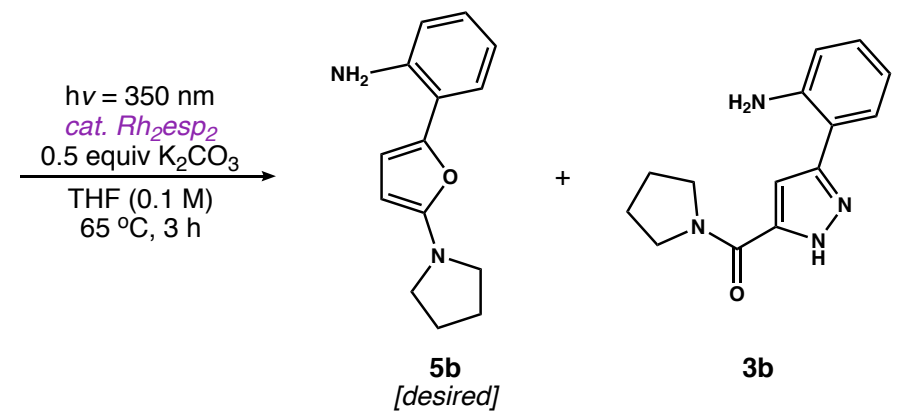

\begin{tabular}{ccc} 
entry & $\mathrm{mol} \% \mathrm{Rh}_{2} \mathrm{esp}_{2}$ & ratio $\mathbf{5 b}: \mathbf{3 b}$ \\
1 & none & $1: 19$ \\
2 & 1.5 & $2: 1$ \\
3 & 2 & $8: 1$ \\
4 & 2.5 & $17: 1$ \\
5 & 3 & $>20: 1$ \\
\hline
\end{tabular}


Representative ${ }^{1} \mathrm{H}$ NMR (Table S1, entry 2)

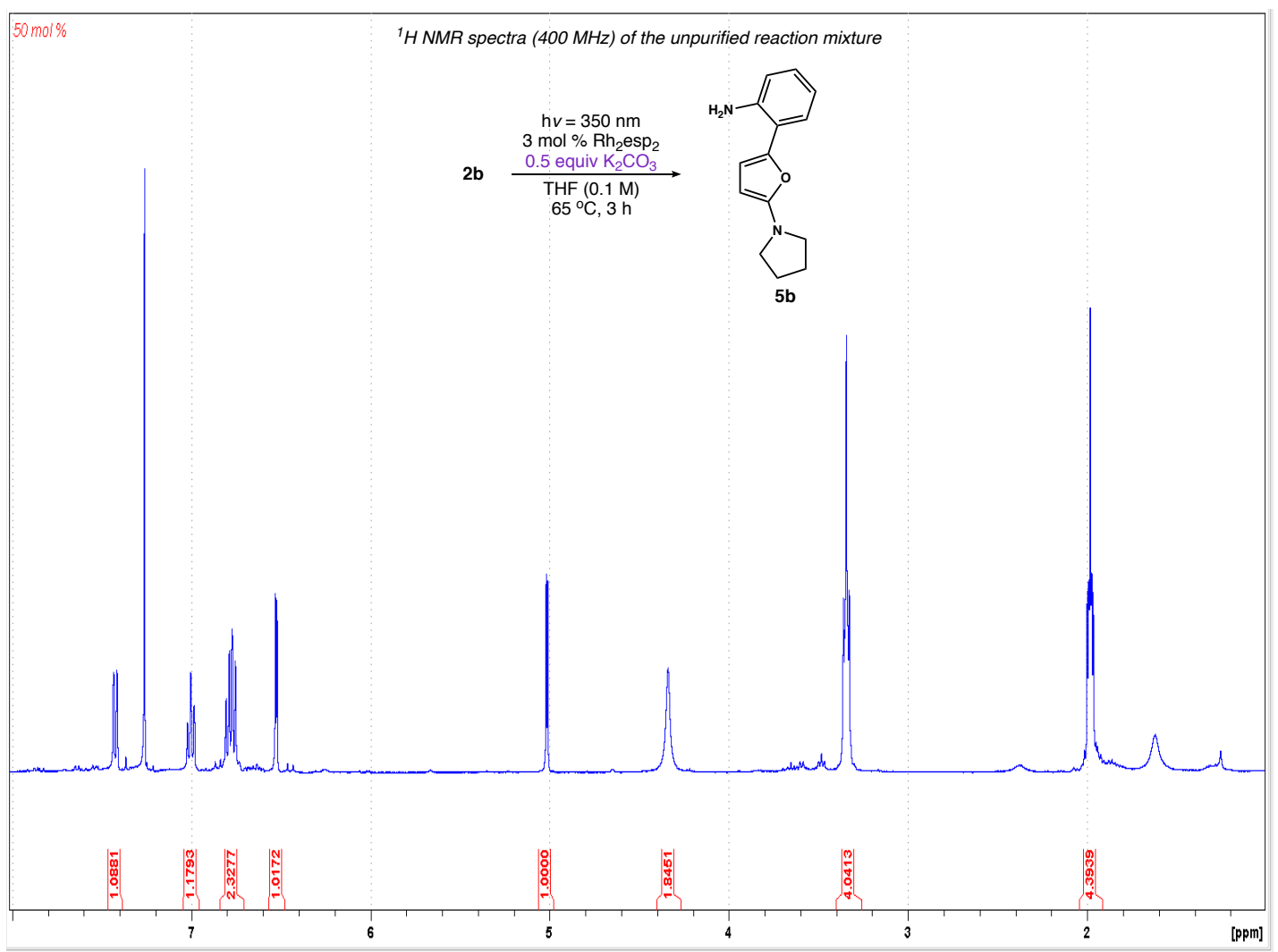

\section{Half-Life Stability Experiments for $5 a$ and $5 b$.}

Procedure (5a): An NMR tube was charged with a solution of $5 \mathrm{a}(2.3 \mathrm{mg}, 0.011 \mathrm{mmol})$ and bibenzyl $\left(1.9 \mathrm{mg}, 0.011 \mathrm{mmol}\right.$, internal standard) in $\mathrm{CDCl}_{3}(0.45 \mathrm{~mL})$. A ${ }^{1} \mathrm{H}$ NMR spectra of the reaction mixture was obtained (time $=0 \mathrm{~h}, 100 \% \mathbf{5 a}$ ). Decomposition was then determined by integration of 5 a relative to bibenzyl at various time points over $31 \mathrm{~h}$.

Analysis: We determined a half-life $\left(t_{1 / 2}\right)$ of $21.2 \mathrm{~h}$ for $\mathbf{5 a}$ at $\mathrm{rt}$ under ambient conditions. 
Decomposition of 2-aminofuran $5 \mathrm{a}$ in $\mathrm{CDCl}_{3}$ at $\mathrm{rt}-\mathrm{t}_{1 / 2}=21 \mathrm{~h}$

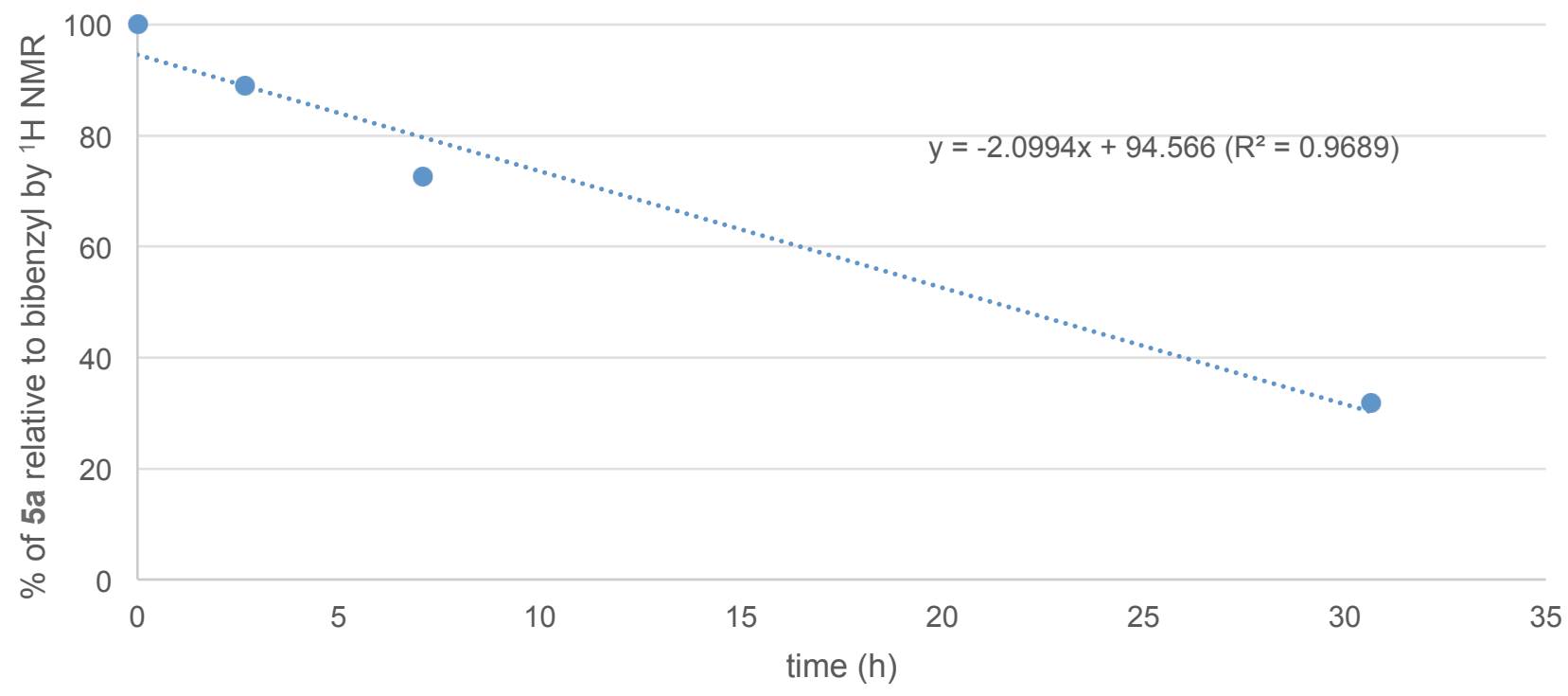

Procedure (5b): An NMR tube was charged with a solution of $5 \mathbf{b}(23 \mathrm{mg}, 0.10 \mathrm{mmol})$ and bibenzyl (18 mg, $0.10 \mathrm{mmol}$, internal standard) in $\mathrm{CDCl}_{3}(0.45 \mathrm{~mL})$. A ${ }^{1} \mathrm{H}$ NMR spectra of the reaction mixture was obtained (time $=0 \mathrm{~h}, 100 \% \mathbf{5 b}$ ). The reaction mixture was then added to a 1-dram vial and concentrated under reduced pressure. The mixture was dissolved in THF $(1 \mathrm{~mL})$ and $\mathrm{K}_{2} \mathrm{CO}_{3}(7 \mathrm{mg}$, $0.05 \mathrm{mmol}$ ) was added. The resulting suspension was added to an aluminum heating block that was pre-warmed to $80{ }^{\circ} \mathrm{C}$. Aliquots $(0.1 \mathrm{~mL})$ were taken at various time points. Each aliquot was concentrated under reduced pressure, dissolved in $\mathrm{CDCl}_{3}(0.45 \mathrm{~mL})$ and analyzed by ${ }^{1} \mathrm{H}$ NMR spectroscopy. Decomposition was determined by integration of $\mathbf{5 b}$ relative to bibenzyl at various time points over $22 \mathrm{~h}$.

Analysis: We determined a half-life $\left(\mathrm{t}_{1 / 2}\right)$ of $13 \mathrm{~h}$ for $5 \mathbf{b}$ at $80{ }^{\circ} \mathrm{C}$ in THF in the presence of $\mathrm{K}_{2} \mathrm{CO}_{3}(0.5$ equiv).

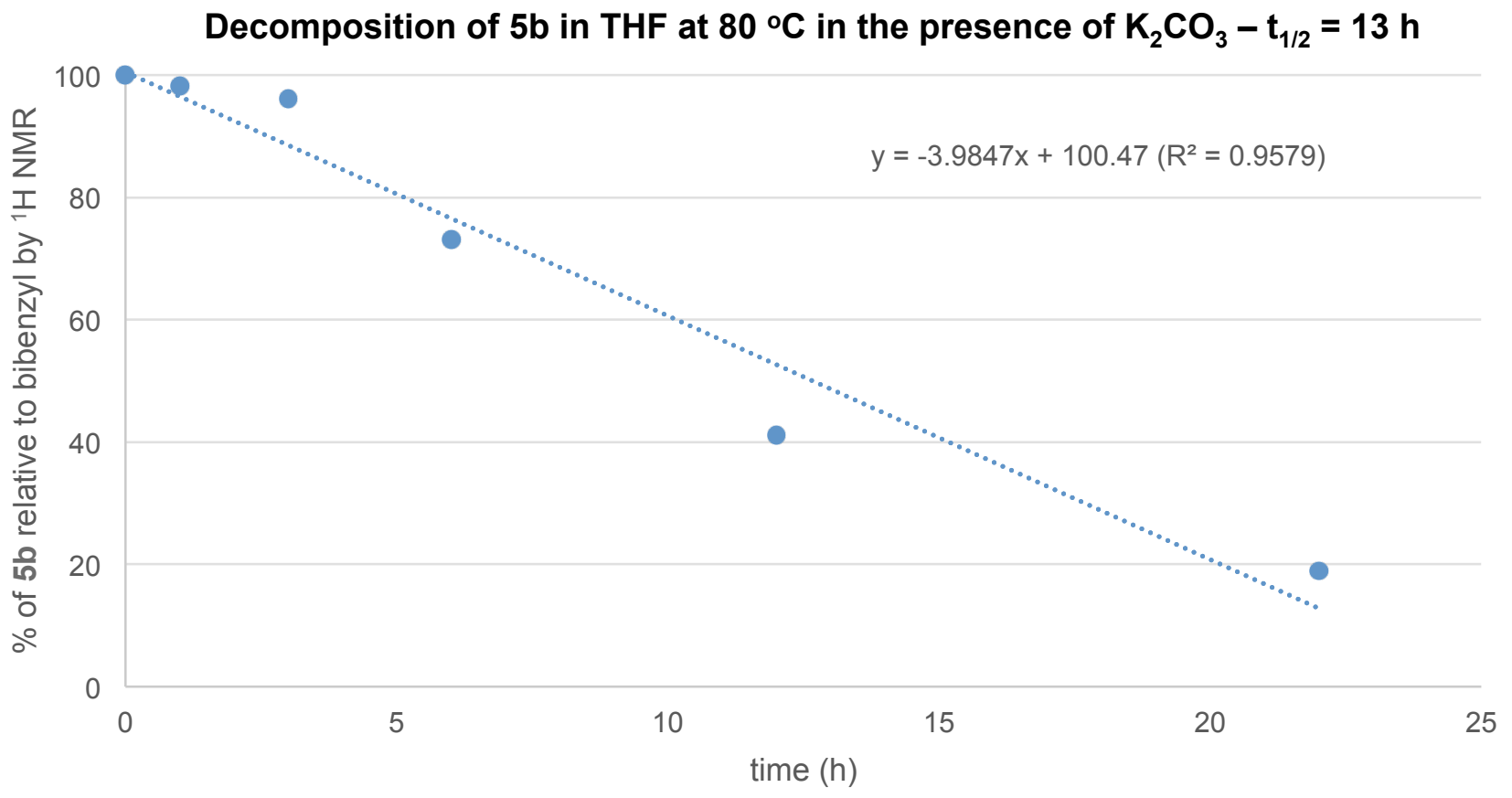




\section{Additional Mechanism Experiments $(5 b \rightarrow 6)$}

Table S3. Mechanism experiments not included in Table 2.<smiles>Nc1ccccc1-c1ccc(N2CCCC2)o1</smiles>

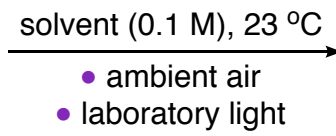

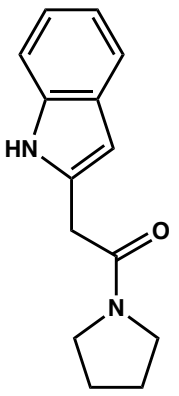

\begin{tabular}{|c|c|c|c|c|}
\hline entry & solvent & modification to conditions & time $(\mathrm{h})$ & $\%$ conversion of $5 \mathbf{b}$ to $\mathbf{6}^{a}$ \\
\hline 1 & $\mathrm{CDCl}_{3}$ & none & 6 & 82 \\
\hline 2 & $\mathrm{CDCl}_{3}$ & oxygen free & 6 & 83 \\
\hline 3 & $\mathrm{CDCl}_{3}$ & $1 \mathrm{~atm} \mathrm{O}_{2}$ & 6 & 85 \\
\hline 4 & THF- $d_{6}$ & none & 6 & 4 \\
\hline 5 & $\mathrm{C}_{6} \mathrm{D}_{6}$ & none & 6 & 9 \\
\hline 6 & $\mathrm{CD}_{3} \mathrm{CN}$ & none & 6 & 10 \\
\hline $7^{b}$ & $\mathrm{CD}_{3} \mathrm{OD}$ & none & 6 & trace \\
\hline
\end{tabular}

6

$5 b$<smiles>Nc1ccccc1-c1ccc(N2CCCC2)o1</smiles>

$5 b$

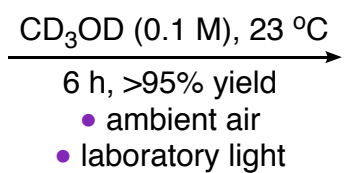

- laboratory light

Procedure: An NMR tube was charged with $5 \mathrm{~b}(13 \mathrm{mg}, 0.044 \mathrm{mmol})$ and bibenzyl $(8 \mathrm{mg}, 0.044$ $\mathrm{mmol}$ ) and $\mathrm{CD}_{3} \mathrm{OD}(0.45 \mathrm{~mL})$. The resulting solution was maintained under ambient conditions and monitored by ${ }^{1} \mathrm{H}$ NMR spectroscopy.

Analysis. The results of this experiment are summarized below. The reaction at $\mathrm{t}=0 \mathrm{~h}$ (top, green) reveals a pair of doublets corresponding to the furan protons $\mathrm{H}_{a}$ and $\mathrm{H}_{\mathrm{b}}$ of $5 \mathbf{b}$. At $t=2 \mathrm{~h}$ (middle, red) the NMR reveals loss of $H_{b}$ and $H_{a}$ becomes a singlet. At $t=6 h$ (bottom, blue) complete $H / D$ exchange for $\mathrm{H}_{\mathrm{b}}$ was observed. 


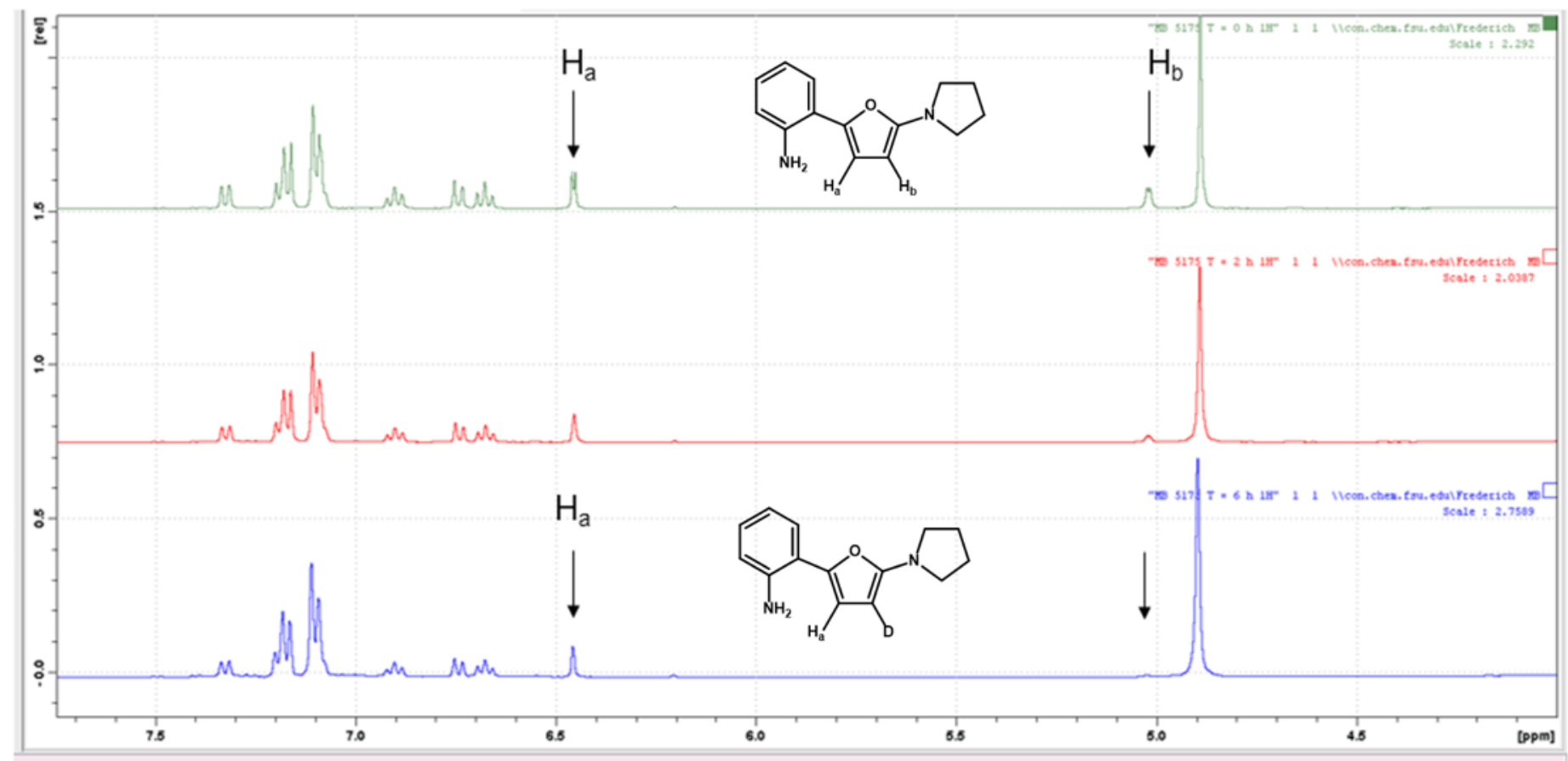

\section{Detailed Experimental Setup - Photochemistry}

Reactions described in this report were carried out in 1-dram borosilicate vials using a Rayonet chamber photoreactor equipped with $24 \mathrm{~W}$ UV-lamps wherein $\sim 90 \%$ of emission is $350 \mathrm{~nm}$. The temperature of the Rayonet is modulated by a built-in fan. The internal temperature holds steady at $35^{\circ} \mathrm{C}$ with the fan on and reaches $65^{\circ} \mathrm{C}$ with the fan off. A series of pictures outlining the conversion of $\mathbf{2 b}$ to $\mathbf{1 3}$ is provided below as a representative reaction.

$\mathbf{A}=1$-dram vials charged with photosubstrate $\mathbf{2 b}, \mathrm{Rh}_{2} \mathrm{esp}_{2}, \mathrm{~K}_{2} \mathrm{CO}_{3}$ in THF. Note that the reaction is heterogeneous.

B $=$ The reaction vials and stir plate were placed within the Rayonet and irradiated using $24 \mathrm{~W}$ lamps for the indicated amount of time.

$\mathbf{C}=\operatorname{In}$ the case of substrate $\mathbf{2 b}$, the photoreaction was complete after $6 \mathrm{~h}$. The unpurified photolysate appeared brown/red and remained heterogeneous.

D = The crude photolysate was cooled to rt. The reaction vessel was then uncapped and treated with 5 equiv of acrylonitrile. The cap was replaced under ambient atmosphere and the reaction mixture was placed on a pre-warmed aluminum block at $80^{\circ} \mathrm{C}$ for $9 \mathrm{~h}$. 

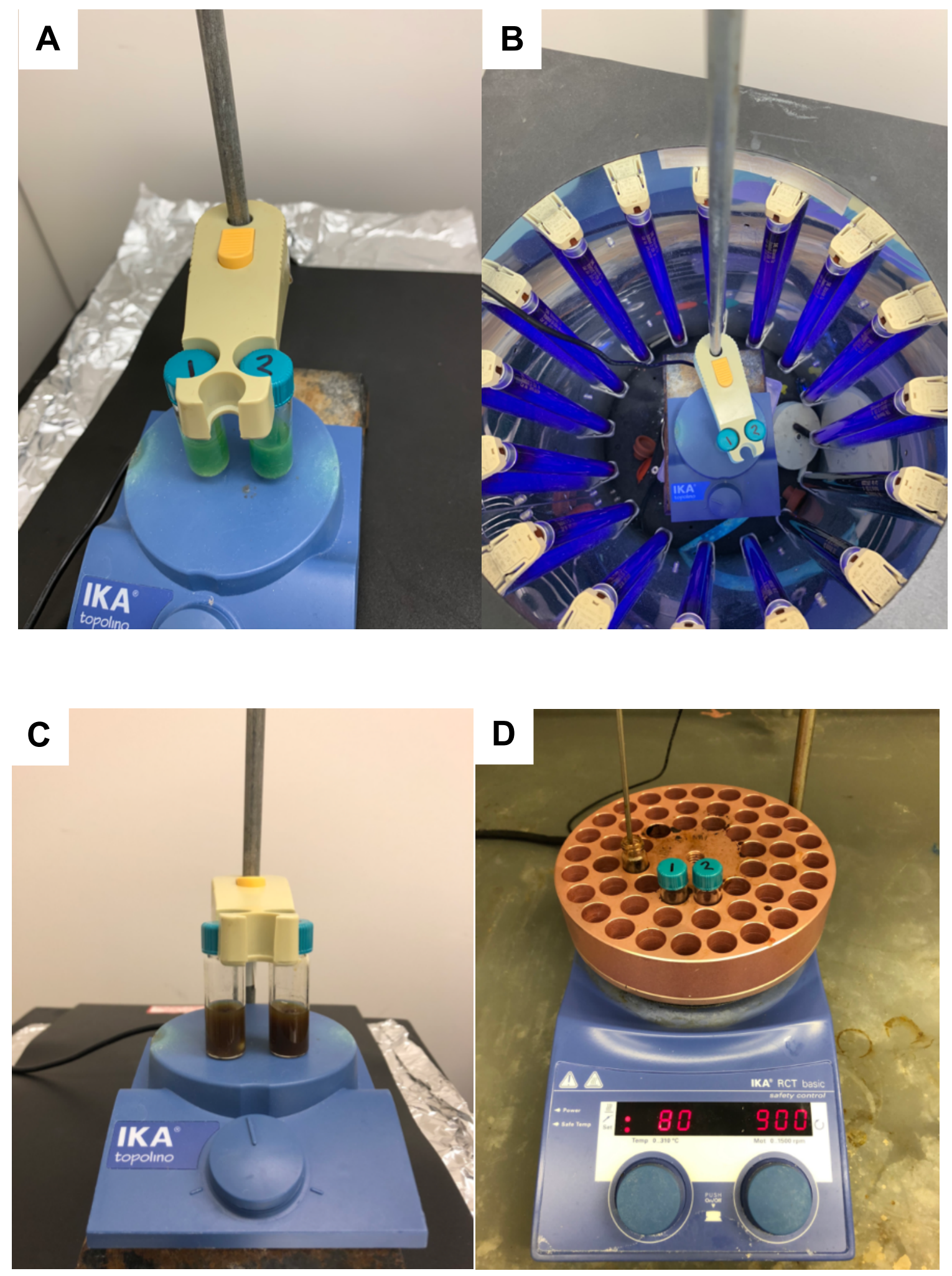
UV-vis spectrum of $\mathbf{2 a}$ was recorded in $\mathrm{MeCN}(0.05 \mathrm{M})$ using an FT-spectrometer. The absorbance spectrum includes $\mathrm{n} \rightarrow \pi^{*}$ band at $\lambda=341 \mathrm{~nm}$.

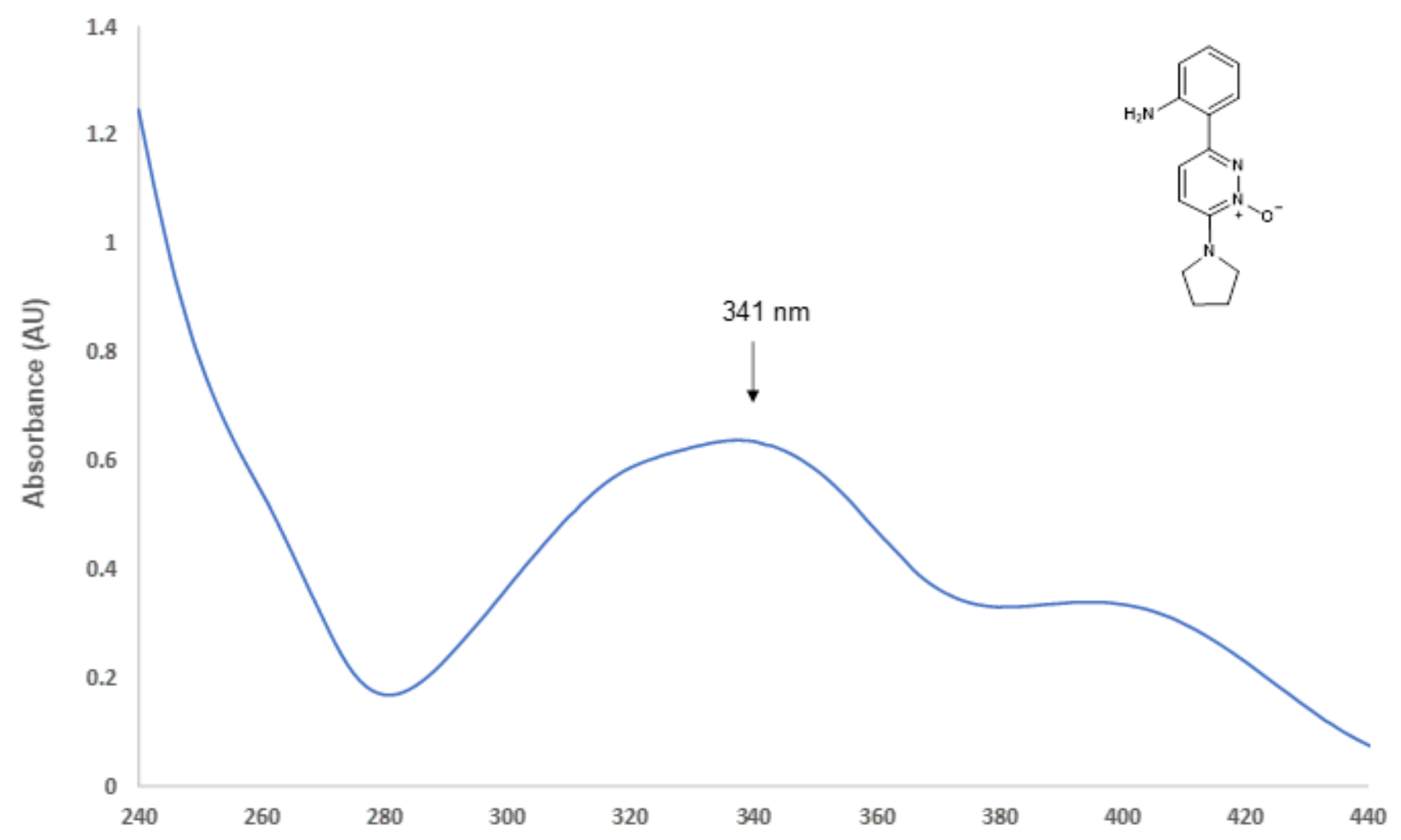

\section{References}

1. Soto, S.; Vaz, E.; Dell'Aversana, C.; Álvarez, R.; Altucci, L.; de Lera, A. R. Org. Biomol. Chem. 2012, 10, 2101.

2. Preshlock, S. M.; Plattner, D. L.; Maligres, P. E.; Krska, S. W.; Maleczka, R. E.; Smith, M. R. Angew. Chem. Int. Ed. 2013, 52, 12915.

3. Friedman, A. A.; Panteleev, J.; Tsoung, J.; Huynh, V.; Lautens, M. Angew. Chem. Int. Ed. 2013, 52, 9755.

4. Sumida, Y.; Kato, T.; Hosoya, T. Org. Lett. 2013, 15, 2806.

5. Longstreet, A. R.; Rivalti, D.; McQuade, T. D. J. Org. Chem. 2015, 80, 8583

6. Portillo, M.; Maxwell, M. A.; Frederich, J. H. Org. Lett. 2016, 18, 5142

7. In many cases the 3-aryl-6-chloropyridazine- $N$-oxides prepared by this procedure appeared to be homogeneous by ${ }^{1} \mathrm{H}$ NMR spectroscopy ahead of purification. Nonetheless, we found that it was critical to purify these compounds by chromatography. Batches of photosubstrates 2 that were not purified by column at this stage often gave poor results when exposed to UV-light. If possible (in rare cases the resulting heterocycle is too insoluble to load on $\mathrm{SiO}_{2}$ ), we strongly recommend a thorough purification at this stage.

8. $\mathrm{K}_{2} \mathrm{CO}_{3}$ was dried in the oven $\left(145^{\circ} \mathrm{C}\right)$ for 24 hours before use and ground into a fine powder using a mortar and pestle. Granulated $\mathrm{K}_{2} \mathrm{CO}_{3}$ used as received gave inferior results. 
9. Reactions were carried out under ambient atmosphere with untreated solvents. We observed no differences when reactions were conducted under an atmosphere of $\mathrm{N}_{2}$ using rigorously degassed solvents.

10. $\mathrm{SiO}_{2}$ was neutralized as follows: Powdered $\mathrm{SiO}_{2}$ was added to a fritted column. The $\mathrm{SiO}_{2}$ pad was then washed with one column volume of EtOAc and one column volume of hexanes. The resultant gel was then washed with one column volume of $95: 5$ hexanes/Et ${ }_{3} \mathrm{~N}$. Unpurified organics were added to silica - typically as solutions in $\mathrm{CH}_{2} \mathrm{Cl}_{2}$ - and eluted using the conditions described.

11. X-ray diffraction data have been deposited at the Cambridge Crystallographic Data Center (CCDC) and can be obtained free of charge at www.ccdc.cam.ac.uk.

12. Zhang, Y.; Yang, X.; Yao, Q.; Ma, D. Org. Lett. 2012, 14, 3056. 


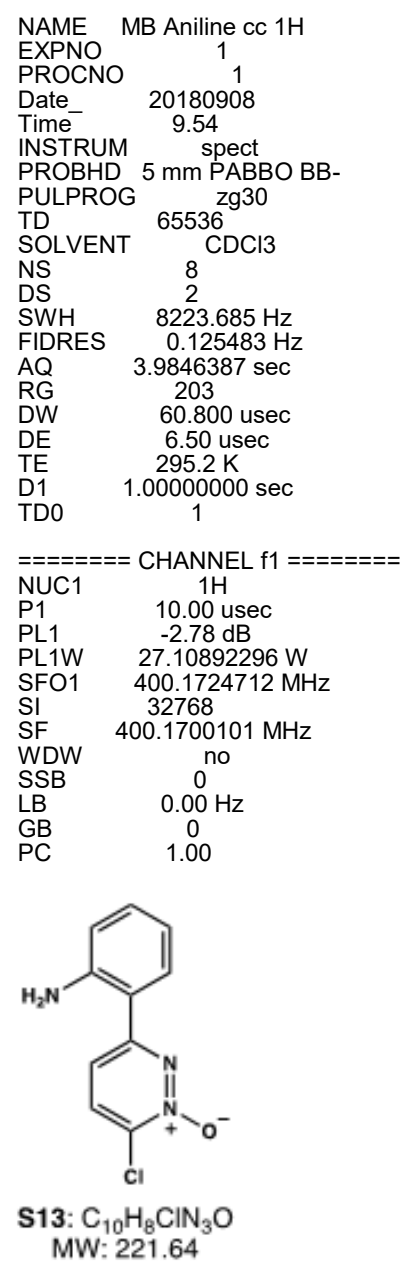

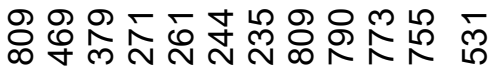

ヘNNヘNN

$\longrightarrow$

PROBHD 5 mm PABBO BB-

PULPROG $\mathrm{Zg} 30$

DS

FIDRES

$========$ CHANNEL $\mathrm{f} 1=======$

SFO1 $\quad 400.1724712 \mathrm{MHz}$

SF $\quad 400.1700101 \mathrm{MHz}$

WDW $\quad 0^{\text {no }}$

GB $\quad 0.00 \mathrm{~Hz}$

1.00
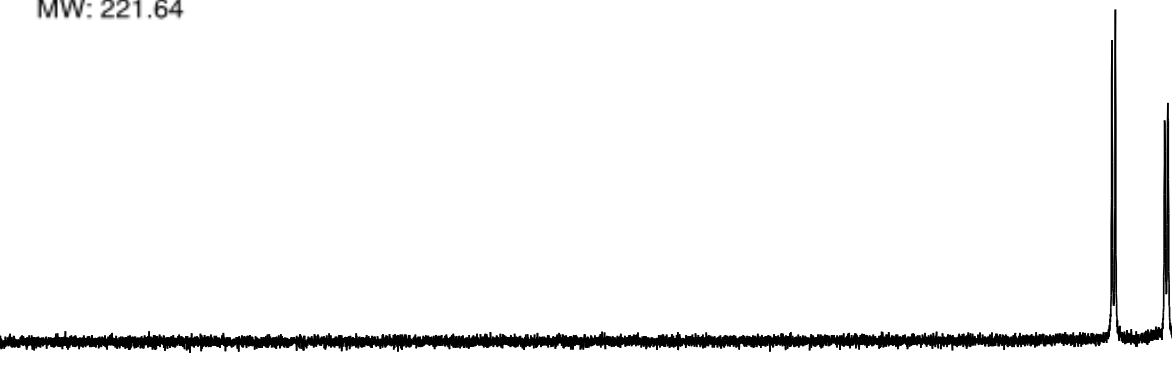

5



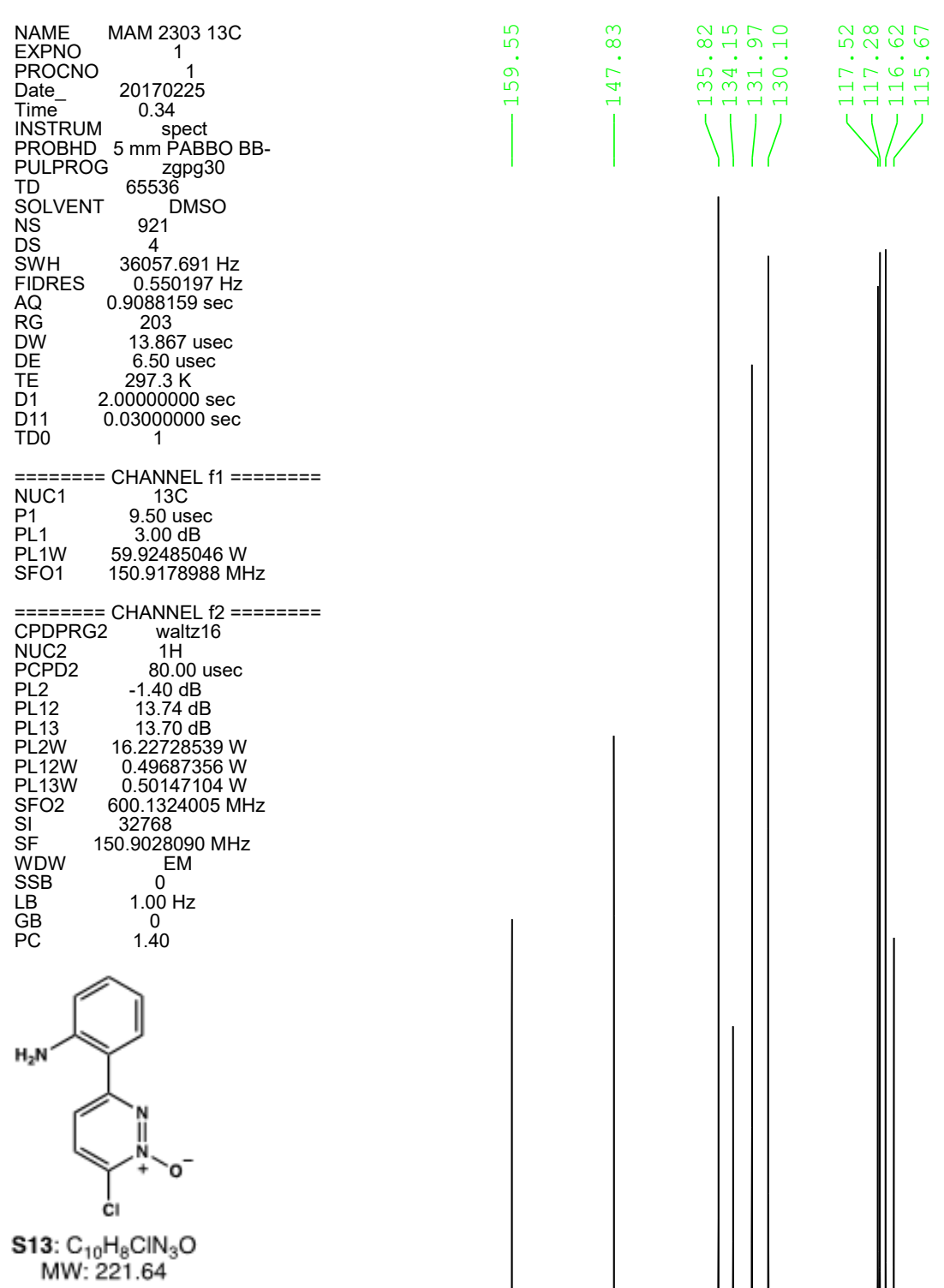

PROBHD $5 \mathrm{~mm}$ PABBO BB-

PULPROG Zgpg30

$=======$ CHANNEL $f 1$ =======

$\quad 9.50$ usec

SFO1 $150.9178988 \mathrm{MHz}$

$======$ CHANNEL $\mathrm{f} 2$

$\begin{array}{ll}\text { PCPD2 } & 1 \mathrm{H} \\ \text { PCo } & -1.00 \mathrm{u}\end{array}$

PL12 $13.74 \mathrm{~dB}$

PL12W $\quad 10.22728539 \mathrm{~W}$

$\begin{array}{ll}\mathrm{PL} 13 \mathrm{~W} & 0.50147104 \mathrm{~W}\end{array}$

$\begin{array}{lll}\mathrm{SI} & 32768 & \mathrm{MHz}\end{array}$

WDW $\quad$ EM

$\begin{array}{ll}\text { GB } & 1.00 \\ \text { GC } & 0 \\ \text { PC } & 1.40\end{array}$

MW: 221.64

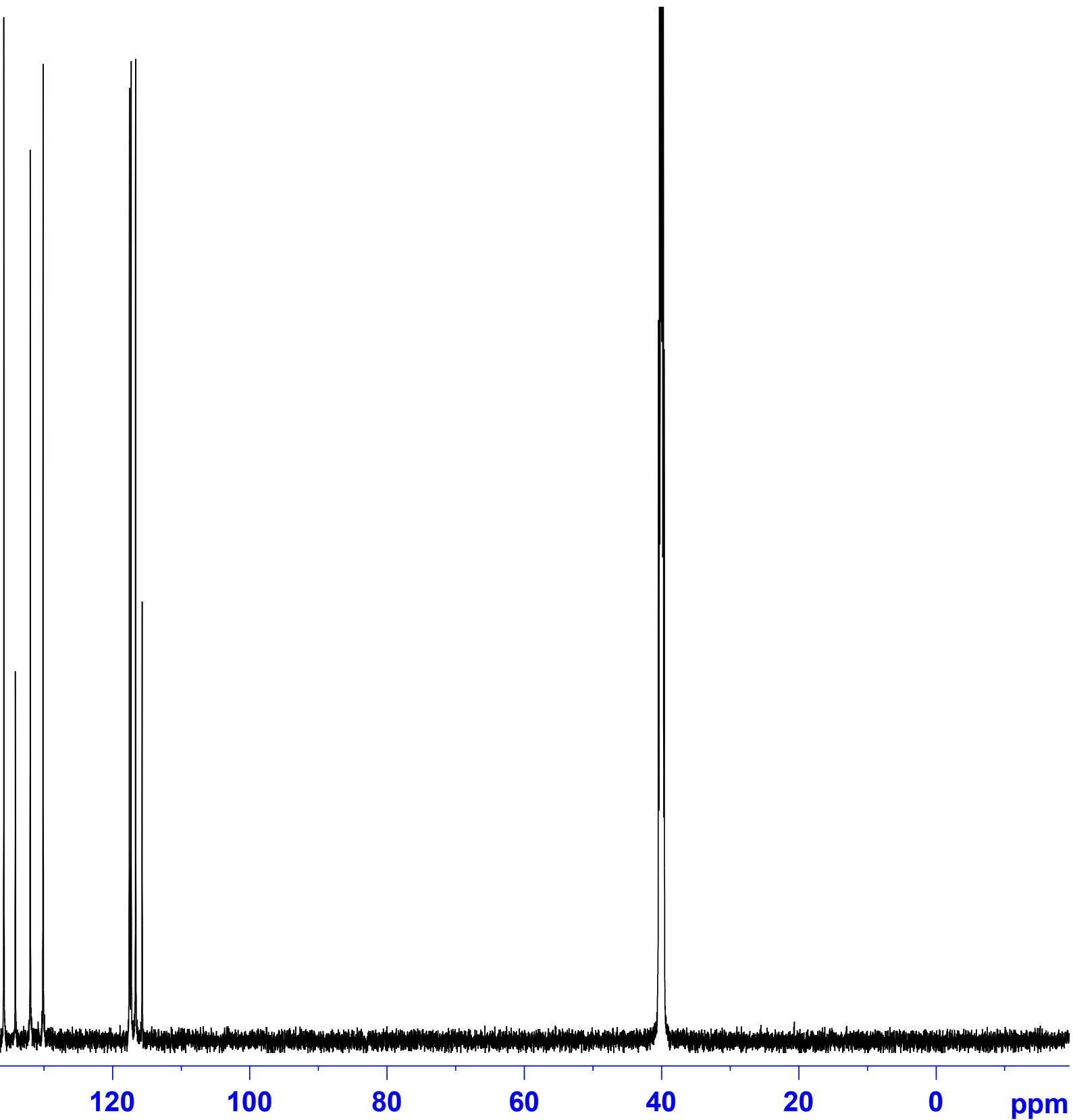




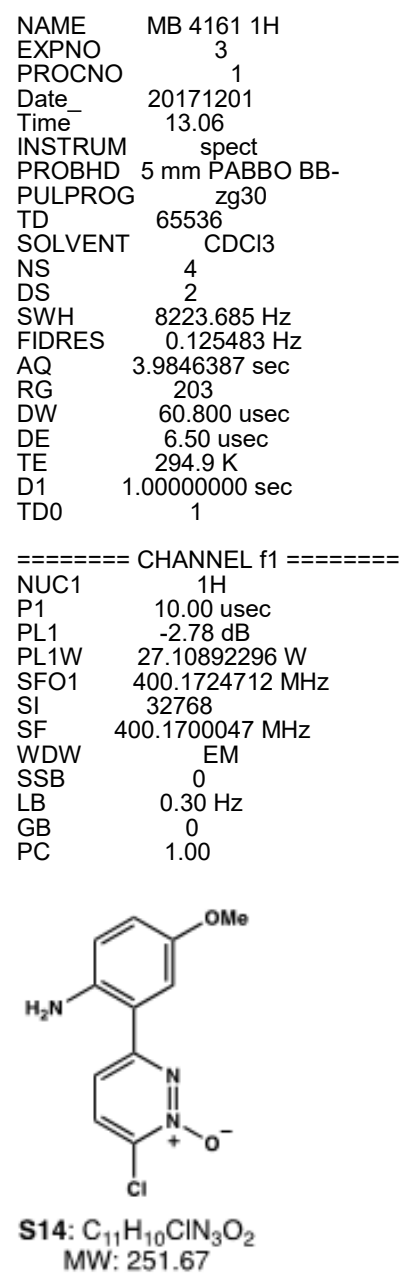

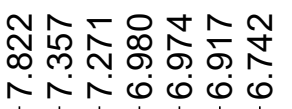

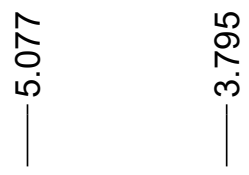

INSTRUM spect

PULPROG $2 \mathrm{~mm}$ zg0

SOLVENT

SWH

$=======$ CHANNEL $f 1$ =======

PC $\quad 1.00$

MW: 251.67

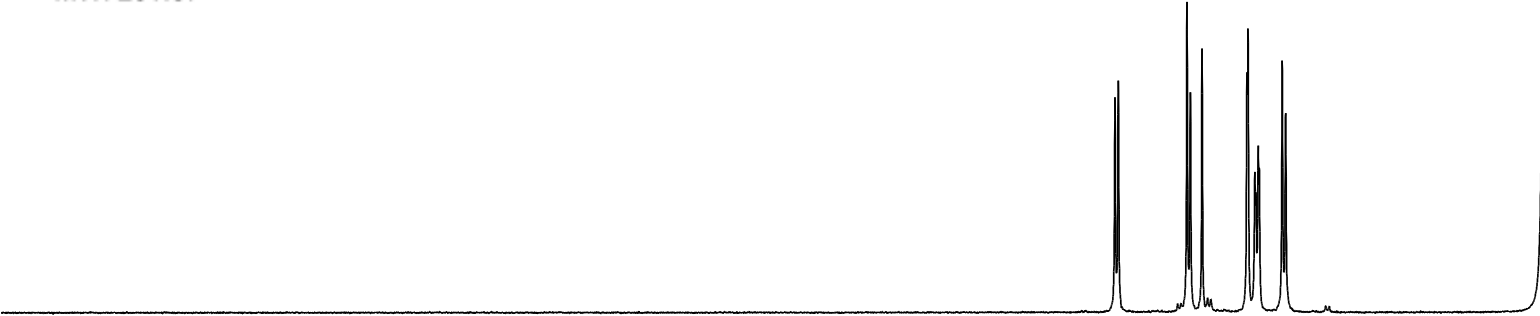

$14 \quad 13$

12

11

10

9

(ํ.

6

埅

बె 


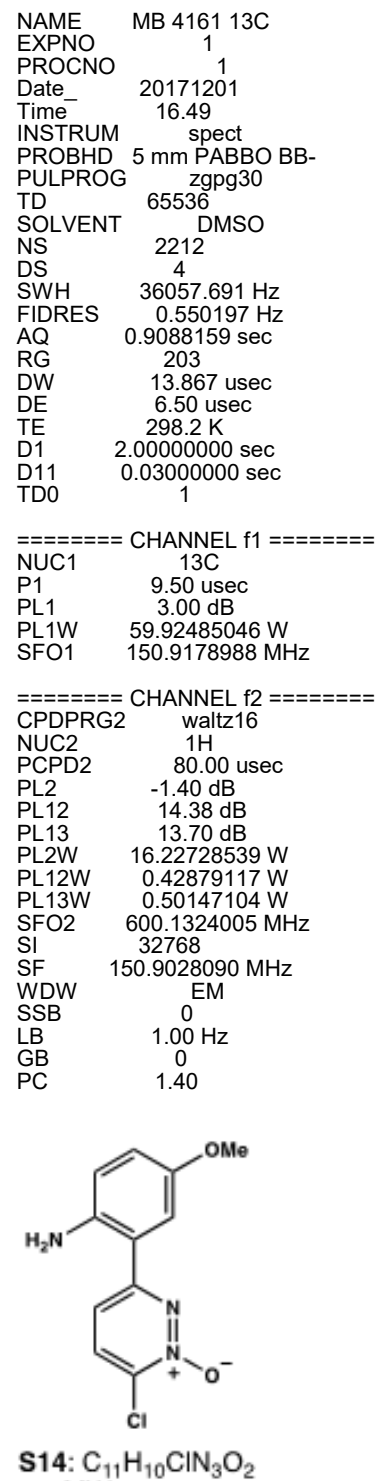

S14: $\mathrm{C}_{11} \mathrm{H}_{10} \mathrm{ClN}_{3} \mathrm{O}_{2}$ MW: 251.67

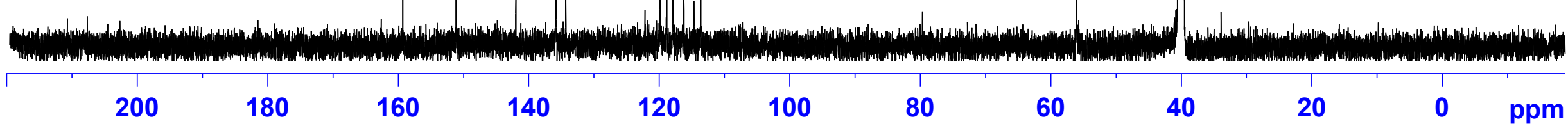




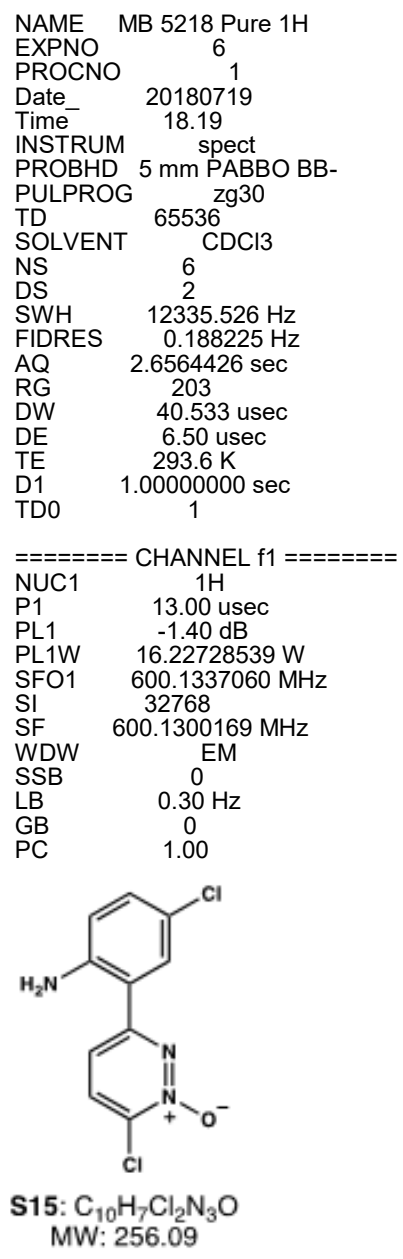

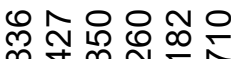

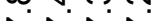

PROCNO 1

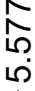

(2)

$=======$ CHANNEL $\mathrm{f} 1$ =======

SFO1 $\quad 600.1337060 \mathrm{MH}$

SF $\quad 600.1300169 \mathrm{MHz}$

WDW

LB $\quad 0.30 \mathrm{~Hz}$

$1 / 1$

14

13

12

11

10

9

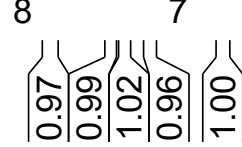

6

5

4

3

2

1 


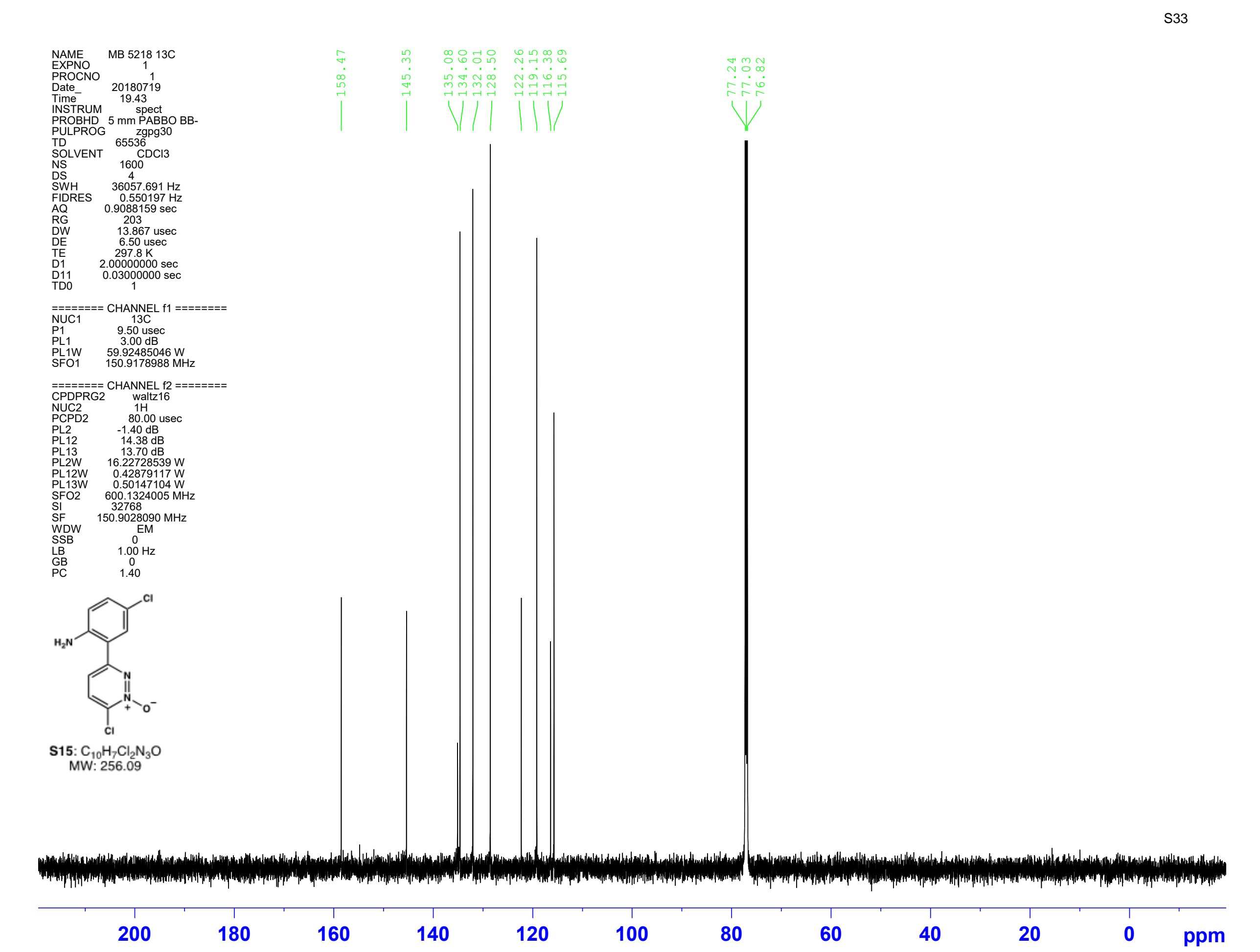




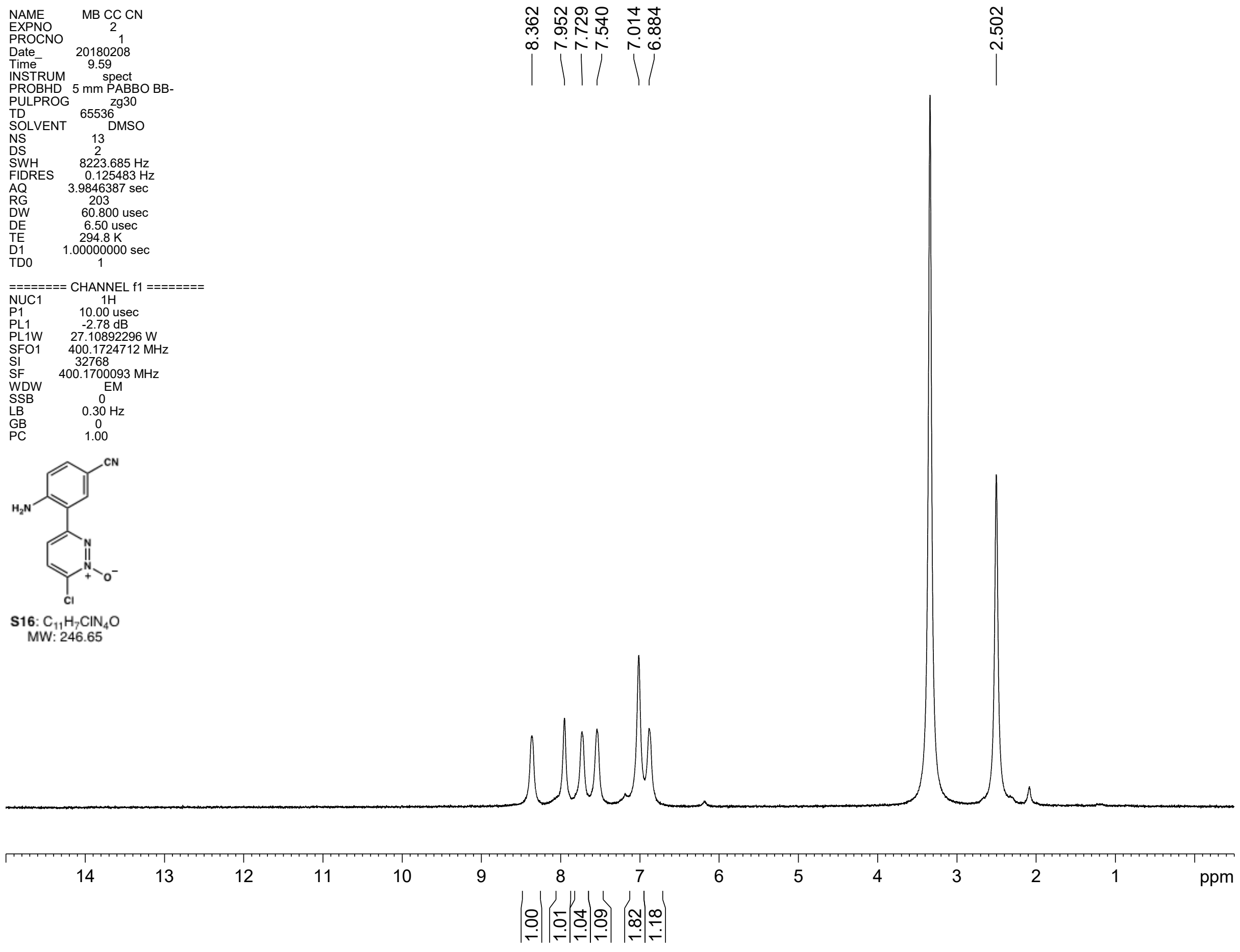



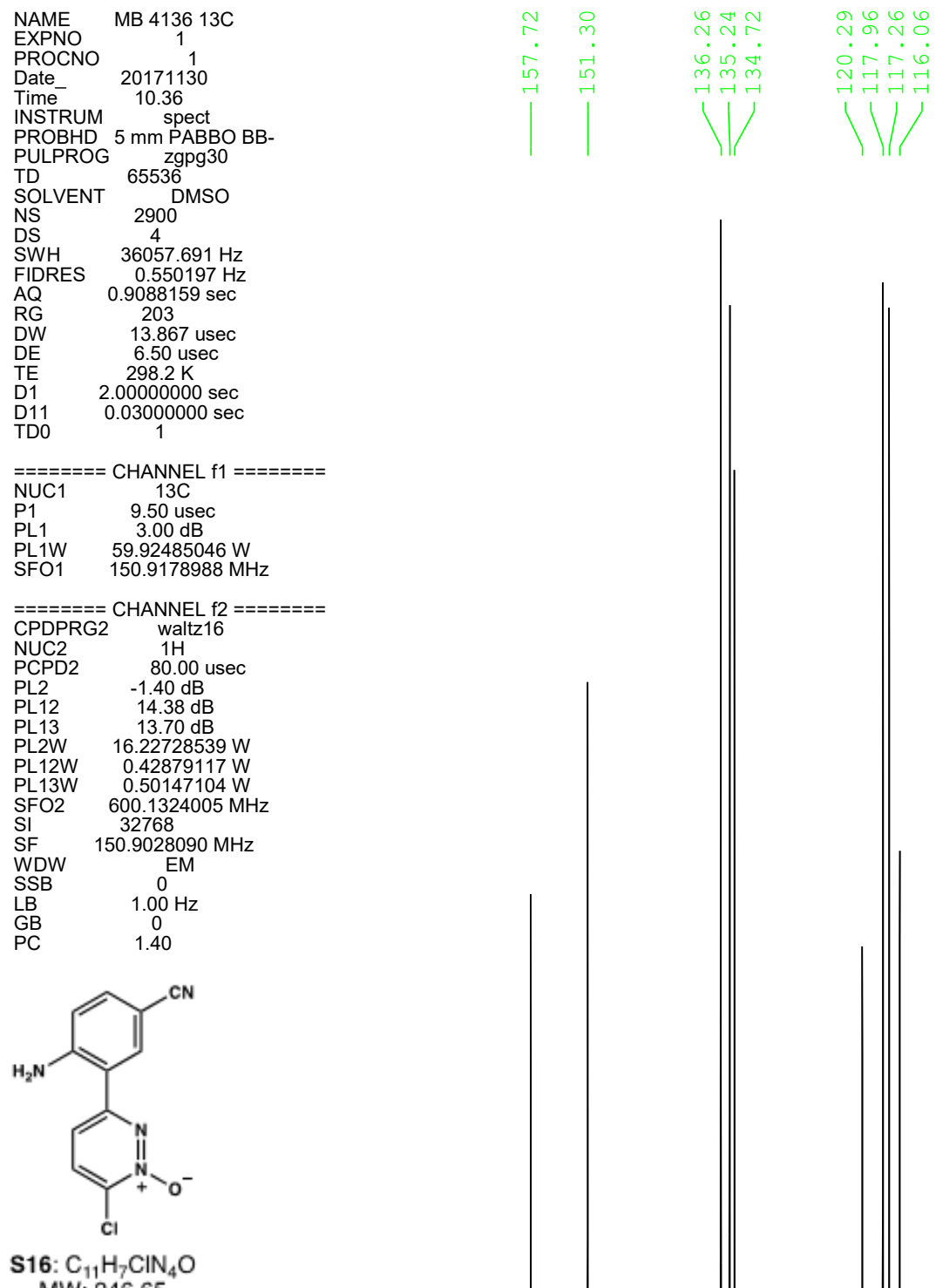

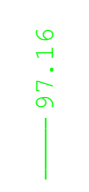

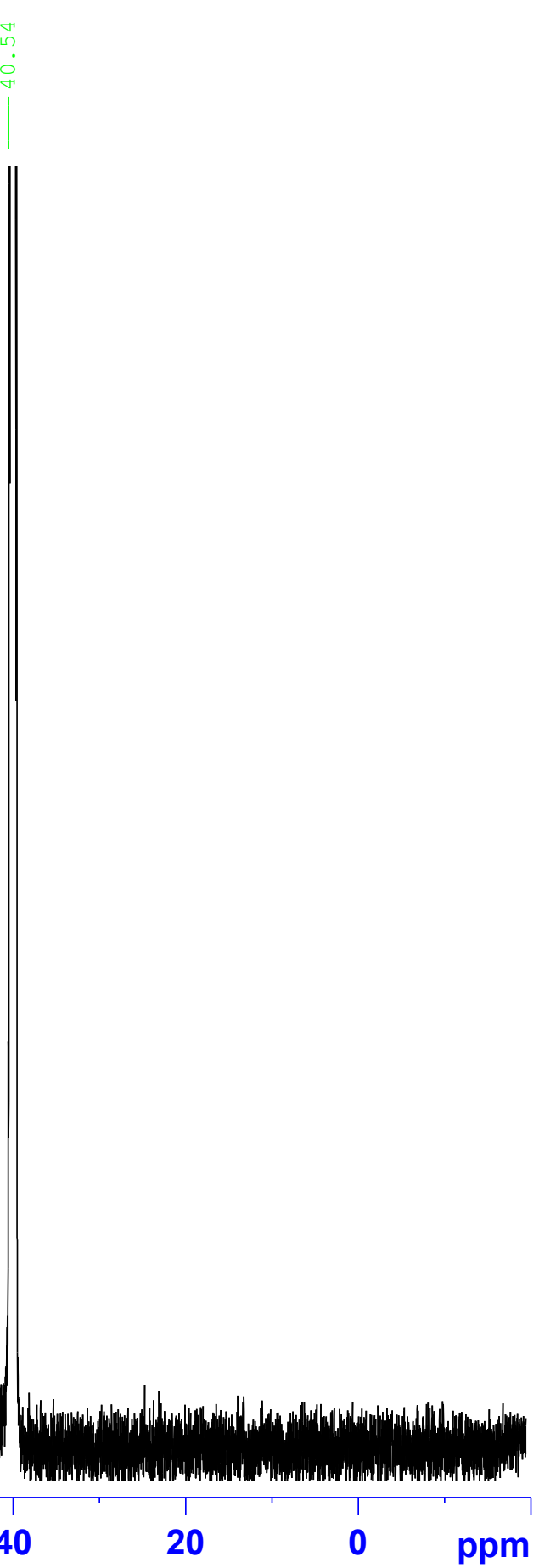




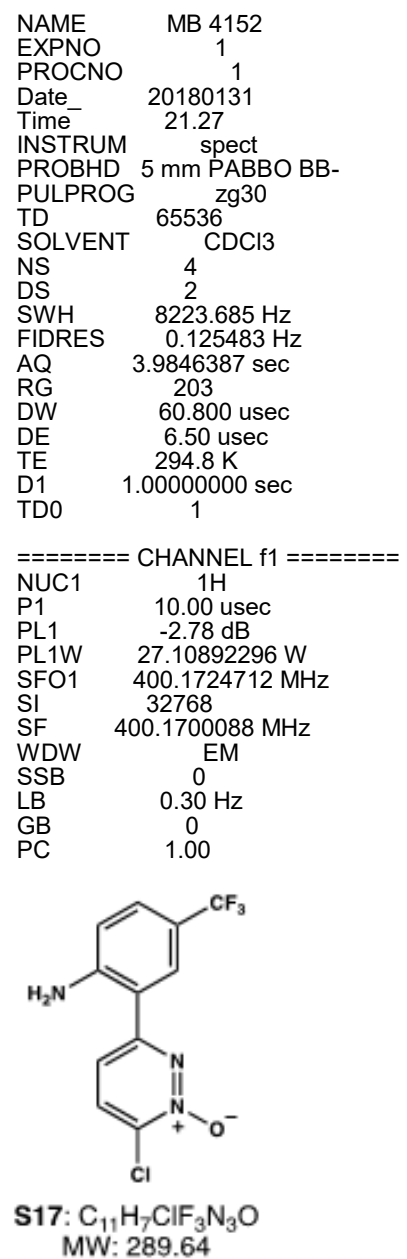

PULPROG $\quad \mathrm{zg} 30$$$
4
$$

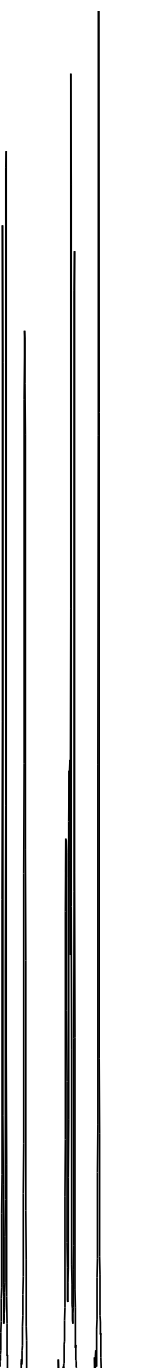

MW: 289.64

14

12

11

9

8

7

6

5

4

3

2 


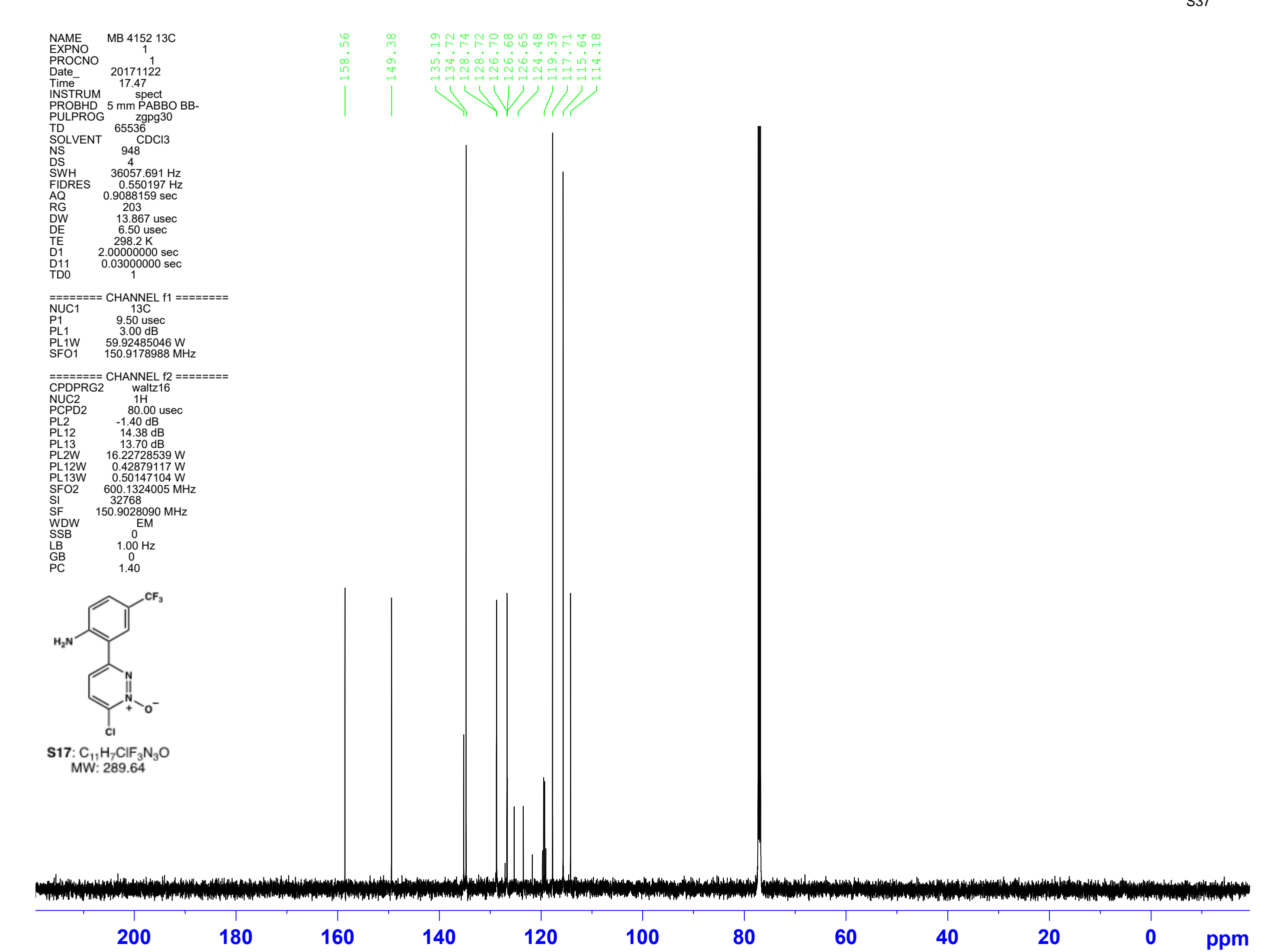



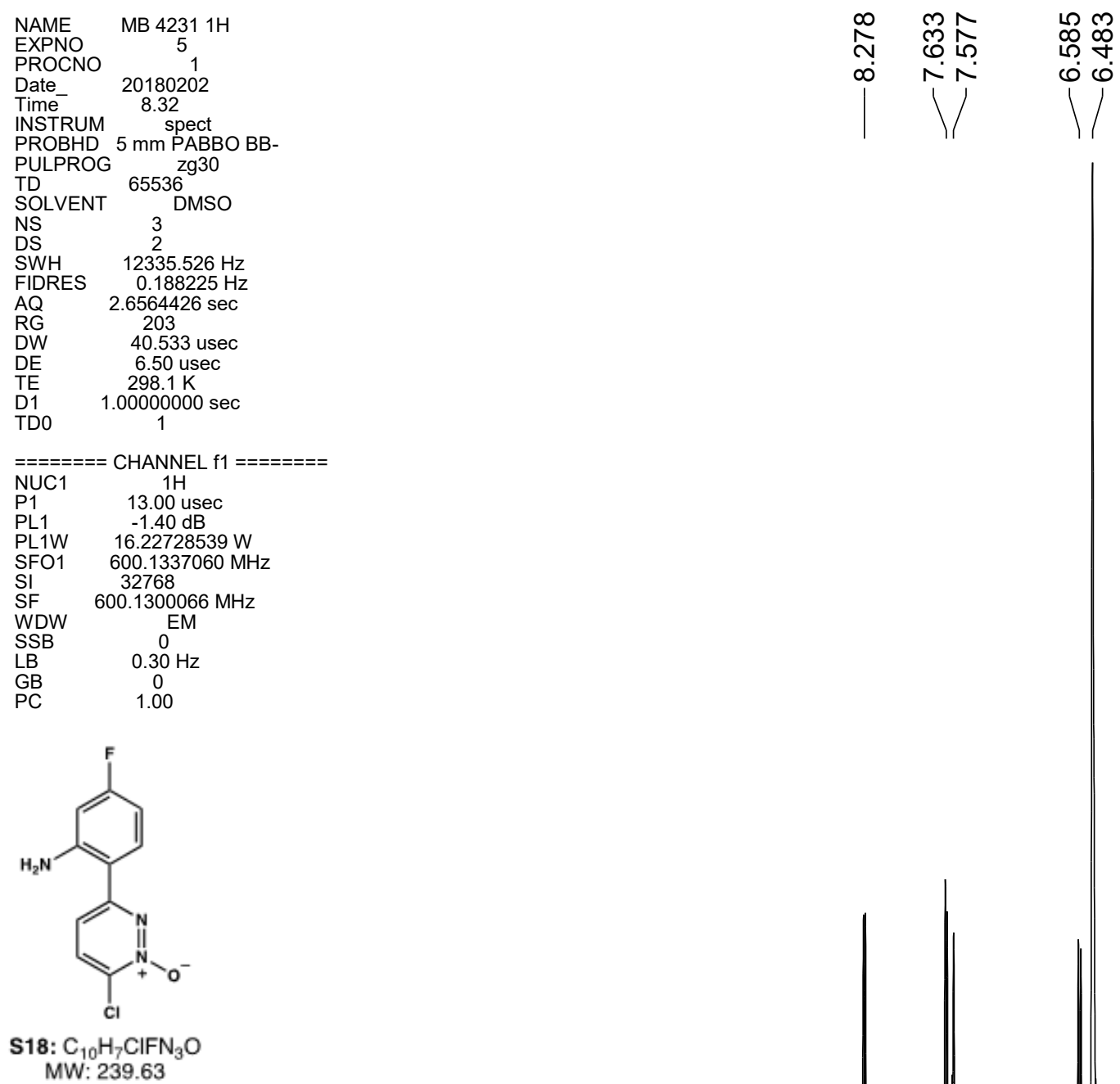

Date 20180202

INSTRUM 8.32

PROBHD $5 \mathrm{~mm}$ PABBO BB-

PULPROG zg30

SOLVENT

DS

SWH $12335.526 \mathrm{~Hz}$

$6.188225 \mathrm{~Hz}$

$=======$ CHANNEL $\mathrm{f} 1$ ========

SFO1 $\quad 600.1337060 \mathrm{MHz}$

SF $\quad 600.1300066 \mathrm{MHz}$

WSW

LB $\quad 0.30 \mathrm{~Hz}$

1.00

MW: 239.63

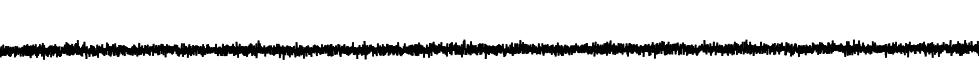

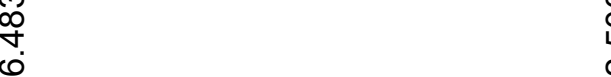

$\uparrow$

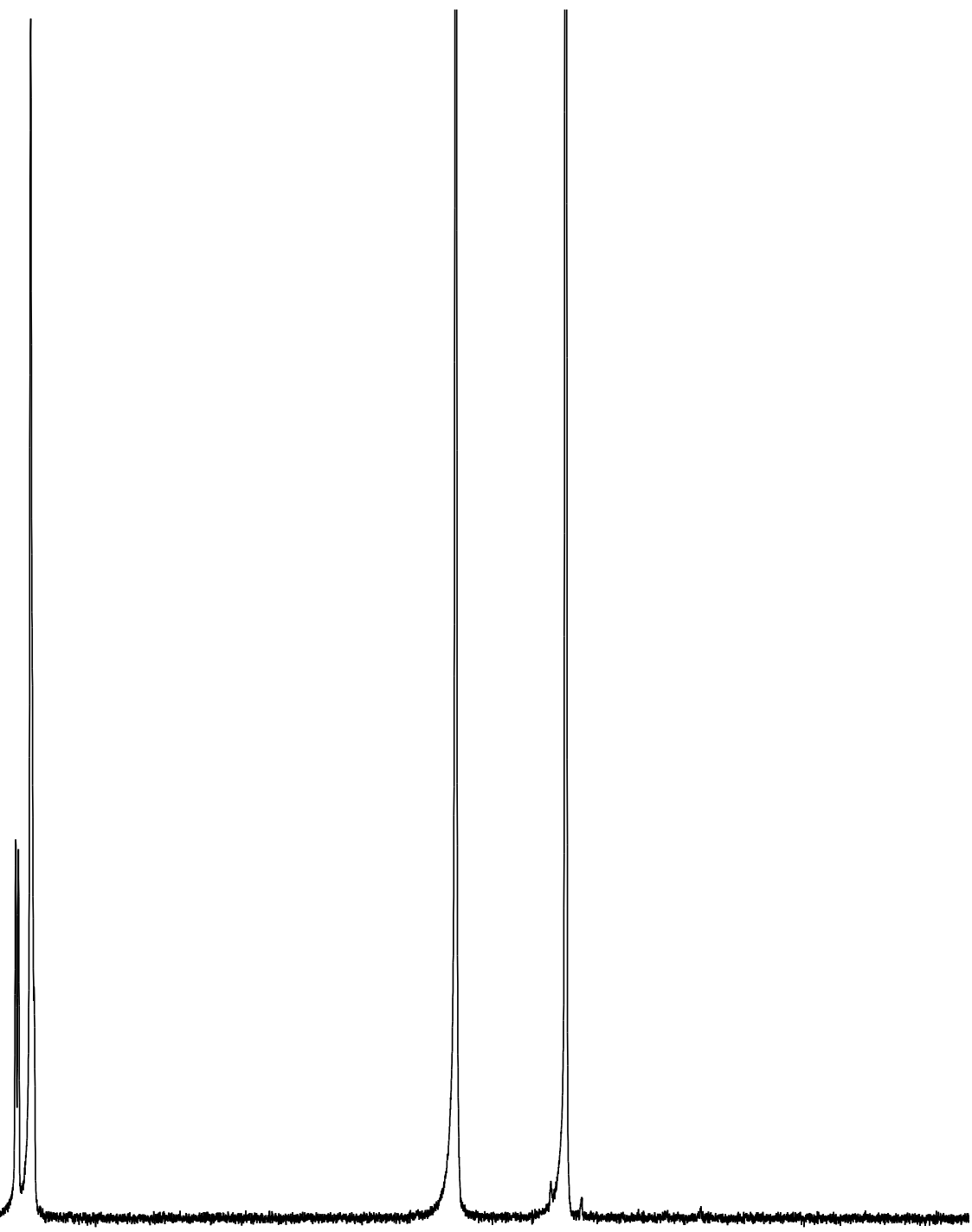

14

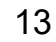

12

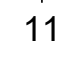

10

9

| 


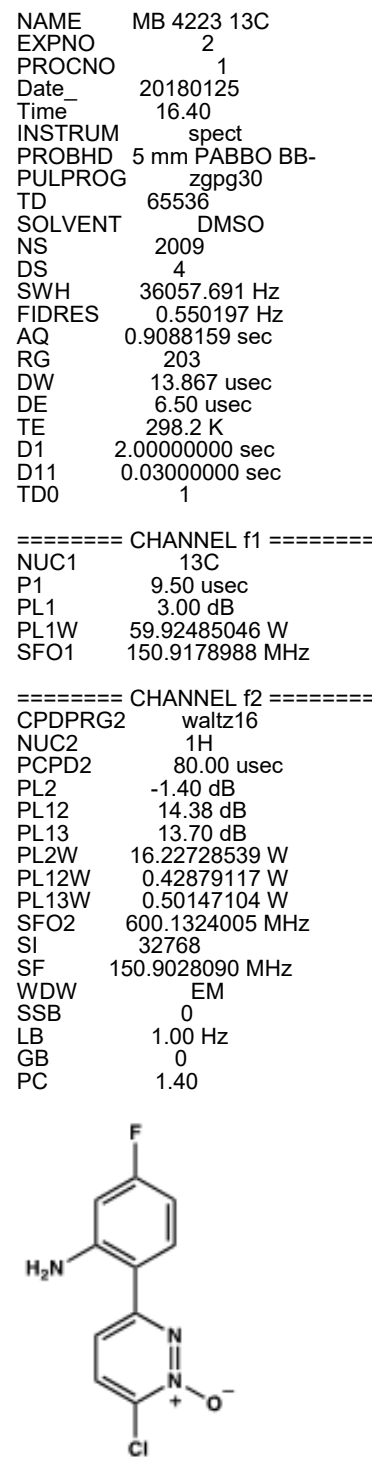

S18: $\mathrm{C}_{10} \mathrm{H}_{7} \mathrm{CIFN}_{3} \mathrm{O}$

MW: 239.63

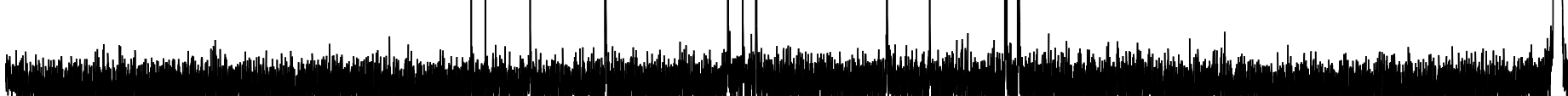

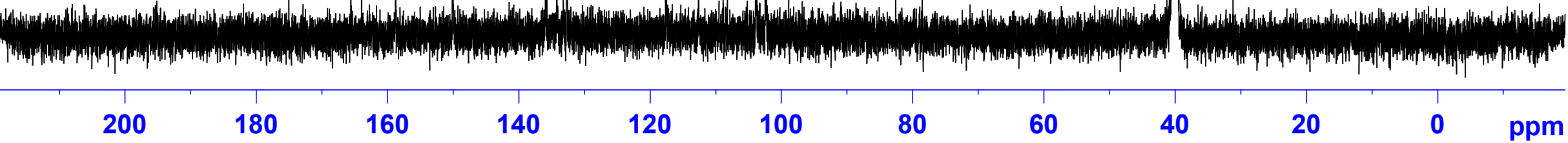




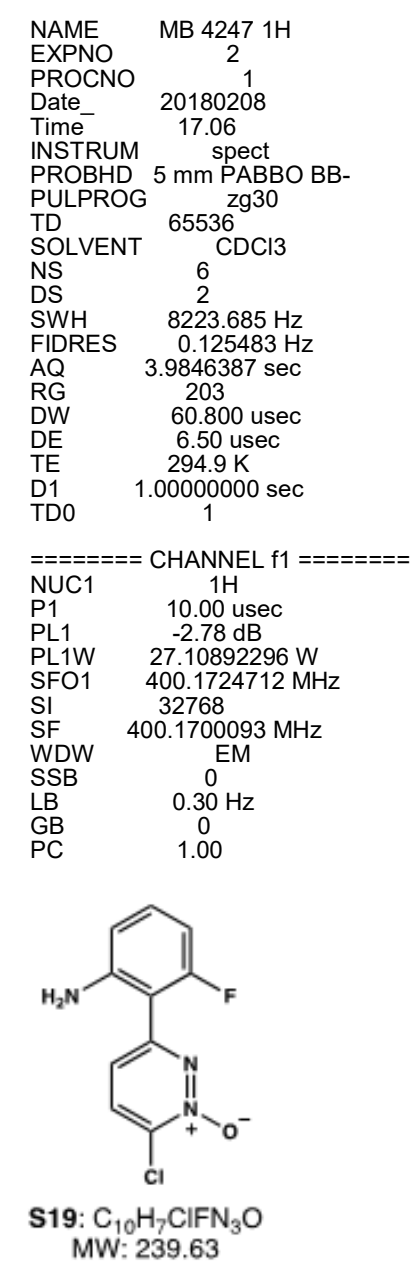

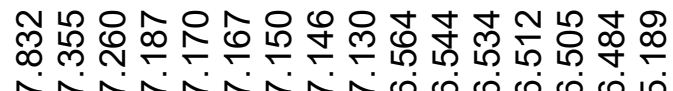

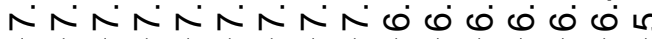

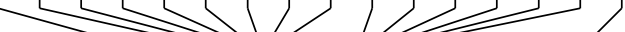




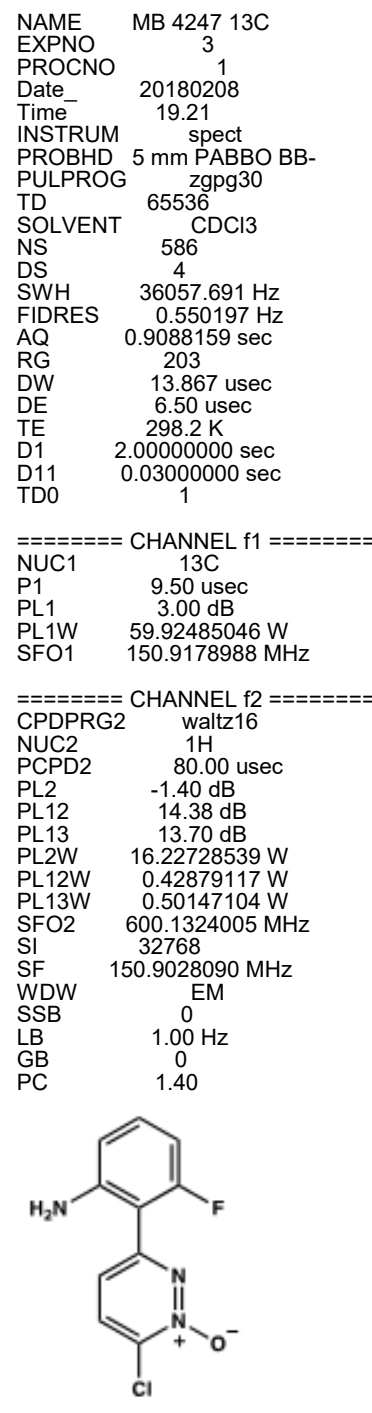

S19: $\mathrm{C}_{10} \mathrm{H}_{7} \mathrm{ClFN}_{3} \mathrm{O}$ MW: 239.63
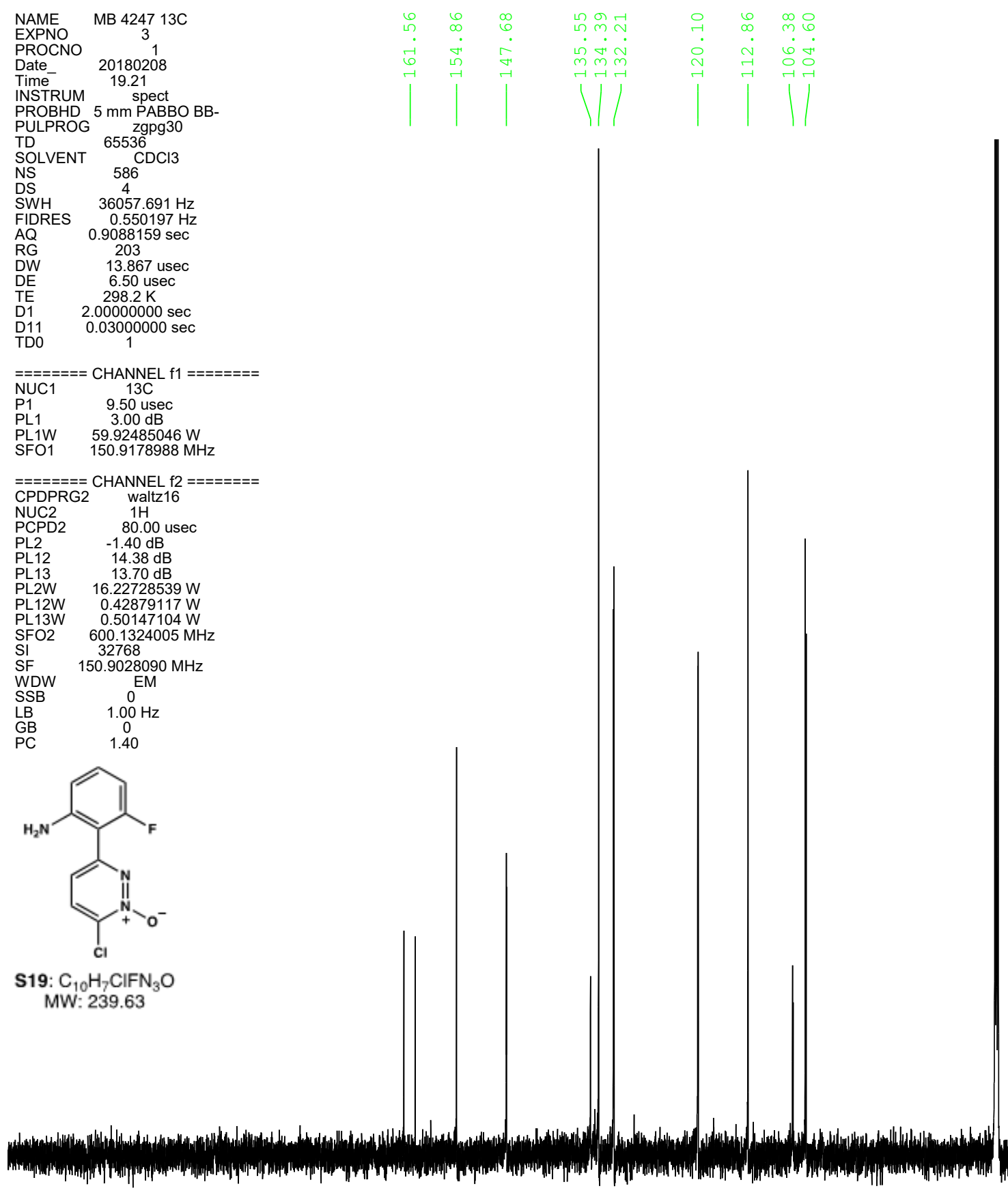

PROBHD $5 \mathrm{~mm}$ PABBO BB-

PULPROG Zggp30

SOLVENT

SWH $\quad 36057.691 \mathrm{~Hz}$

$0.0 .55097 \mathrm{~Hz}$

$\begin{array}{ll}\text { RG } & 203 \\ \text { DW } & 13.567\end{array}$

DE $\quad 6.50$ usec

D1 $2.0000000 \mathrm{sec}$

$===$ CHANNEL $f 1$ =======

$\quad 9.50$ usec

SFO1 $150.9178988 \mathrm{MHz}$

$=======$ CHANNEL

PCPD2 $\quad 80.00$ usec

PL12W $\quad 0.42879117 \mathrm{~W}$

PL13W

SF $\quad 150.9028090 \mathrm{MHz}$

WDW $\quad$ EM

$\begin{array}{ll}\text { GB } & 1.00 \\ \text { PC } & 1.40\end{array}$

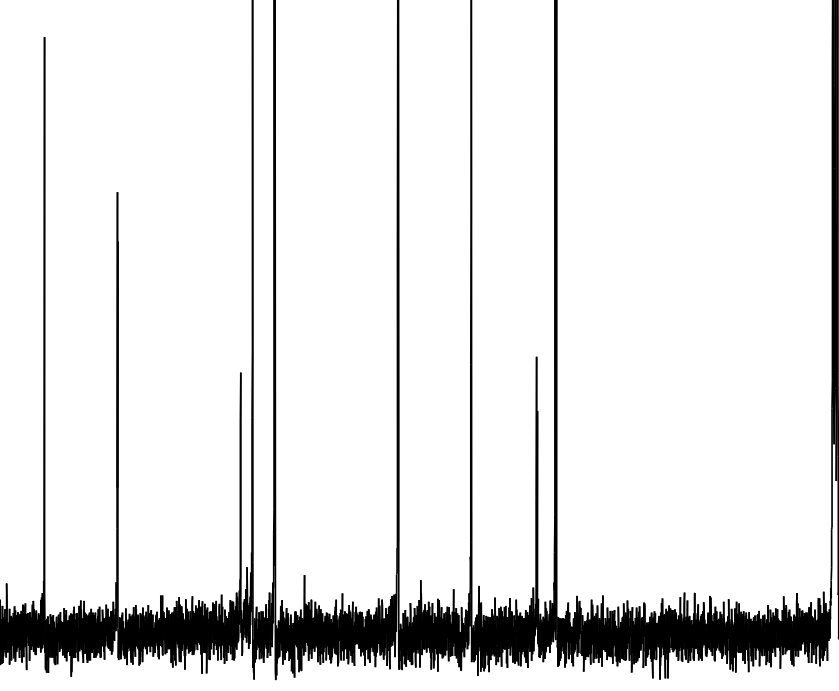



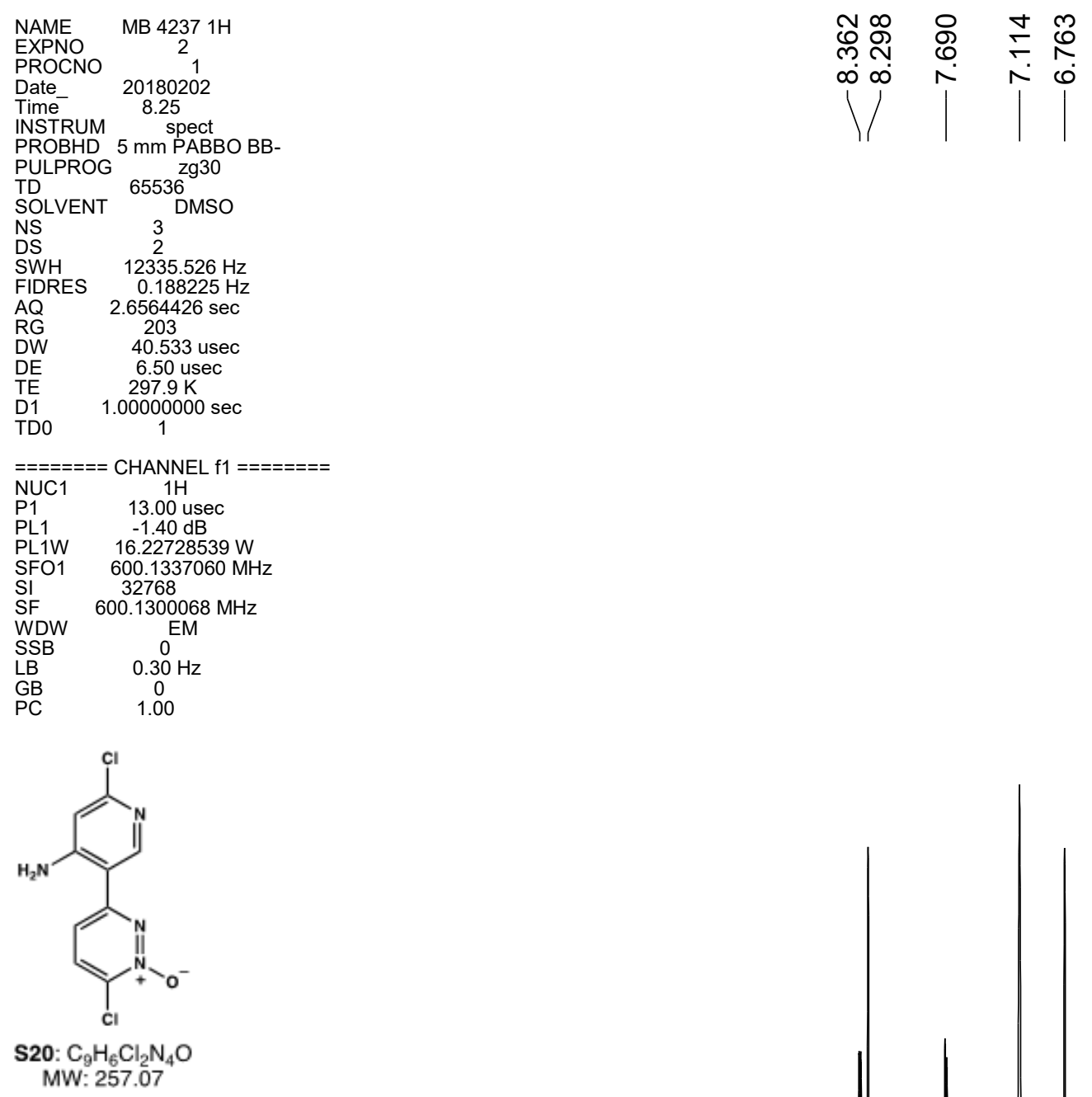

INSTRUM spect

PULPROG 2930

SOLVENT

DS

SWH

203

DE $\quad 6.50$ usec

D1 $1.00000 \mathrm{~K}$

$=======$ CHANNEL $\mathrm{f} 1$ ======="

SFO1 $\quad 600.1337060 \mathrm{MHz}$

SF $\quad 600.1300068 \mathrm{MHz}$

SSB

$\begin{array}{ll}\mathrm{GB} & 0 \\ \mathrm{PC} & 1.00\end{array}$

MW: 257.07
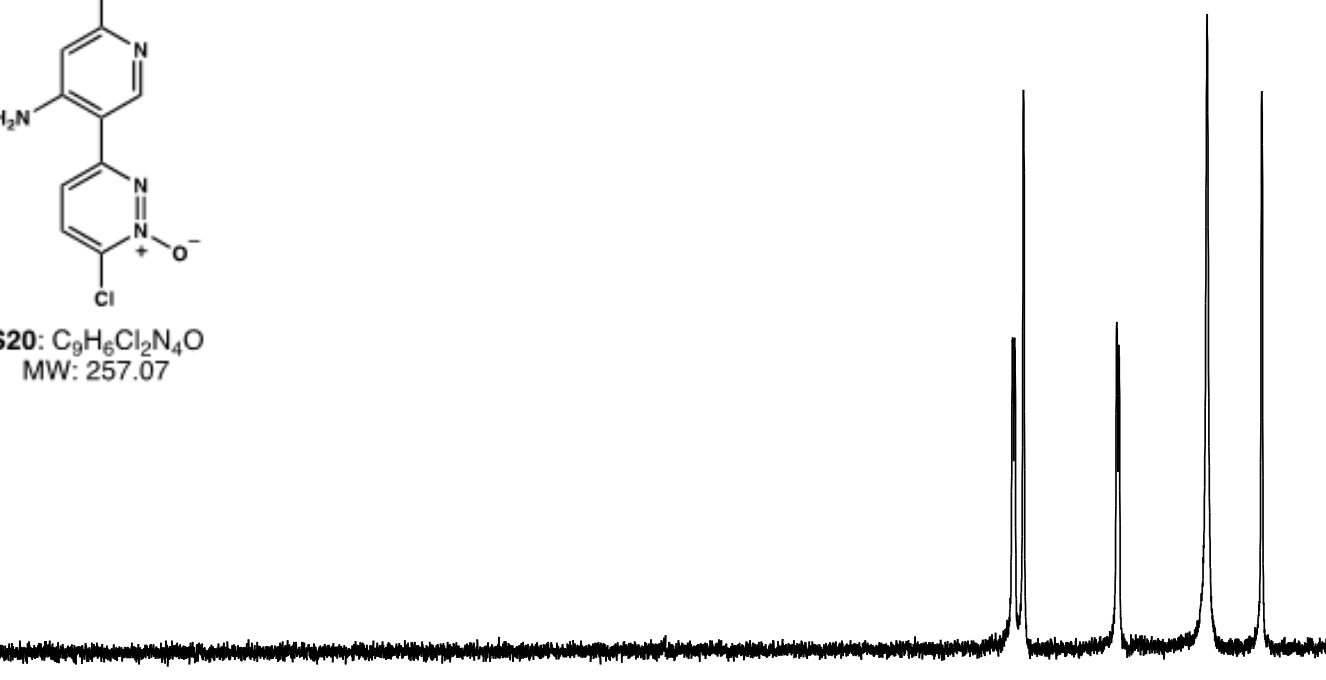

14

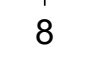

7

6

5

4

3

2

1

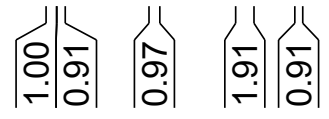



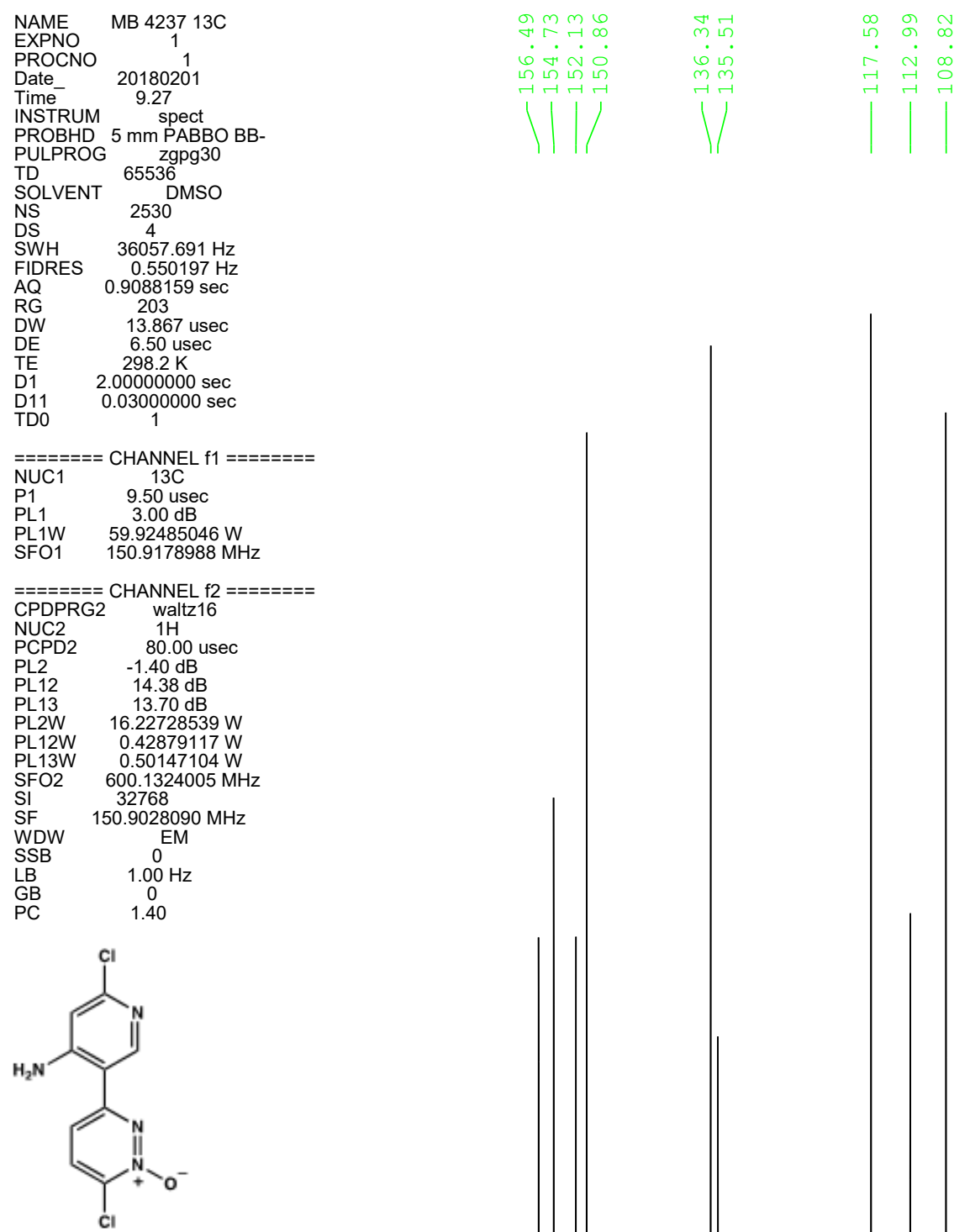

S20: $\mathrm{C}_{9} \mathrm{H}_{6} \mathrm{Cl}_{2} \mathrm{~N}_{4} \mathrm{O}$

MW: 257.07

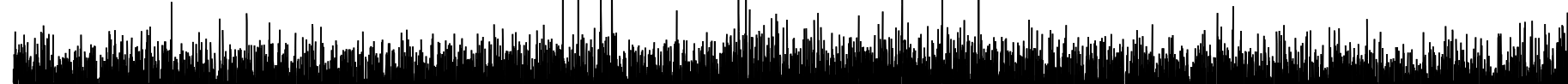

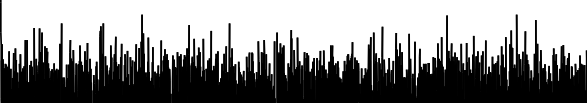

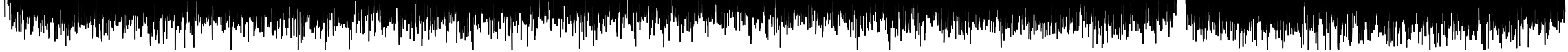

$200 \quad 180$

180

160

140

120

100

80

60

40

20

ppm 


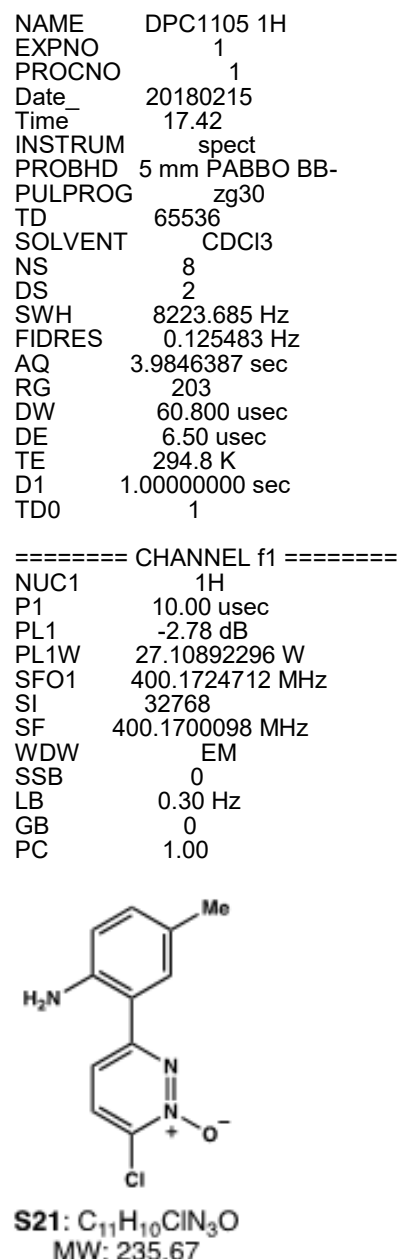

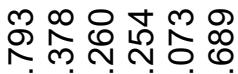

NNNN

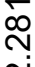

MW: 235.67

14
12

$12 \quad 11$
11

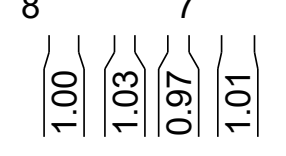
6 $\mid \frac{0}{2}$

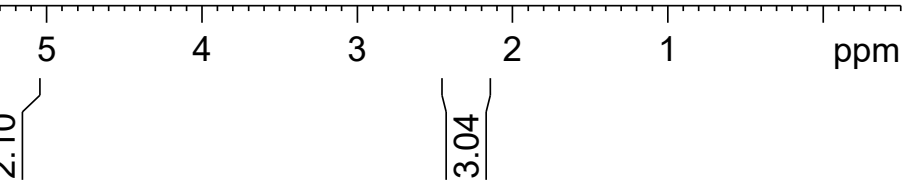



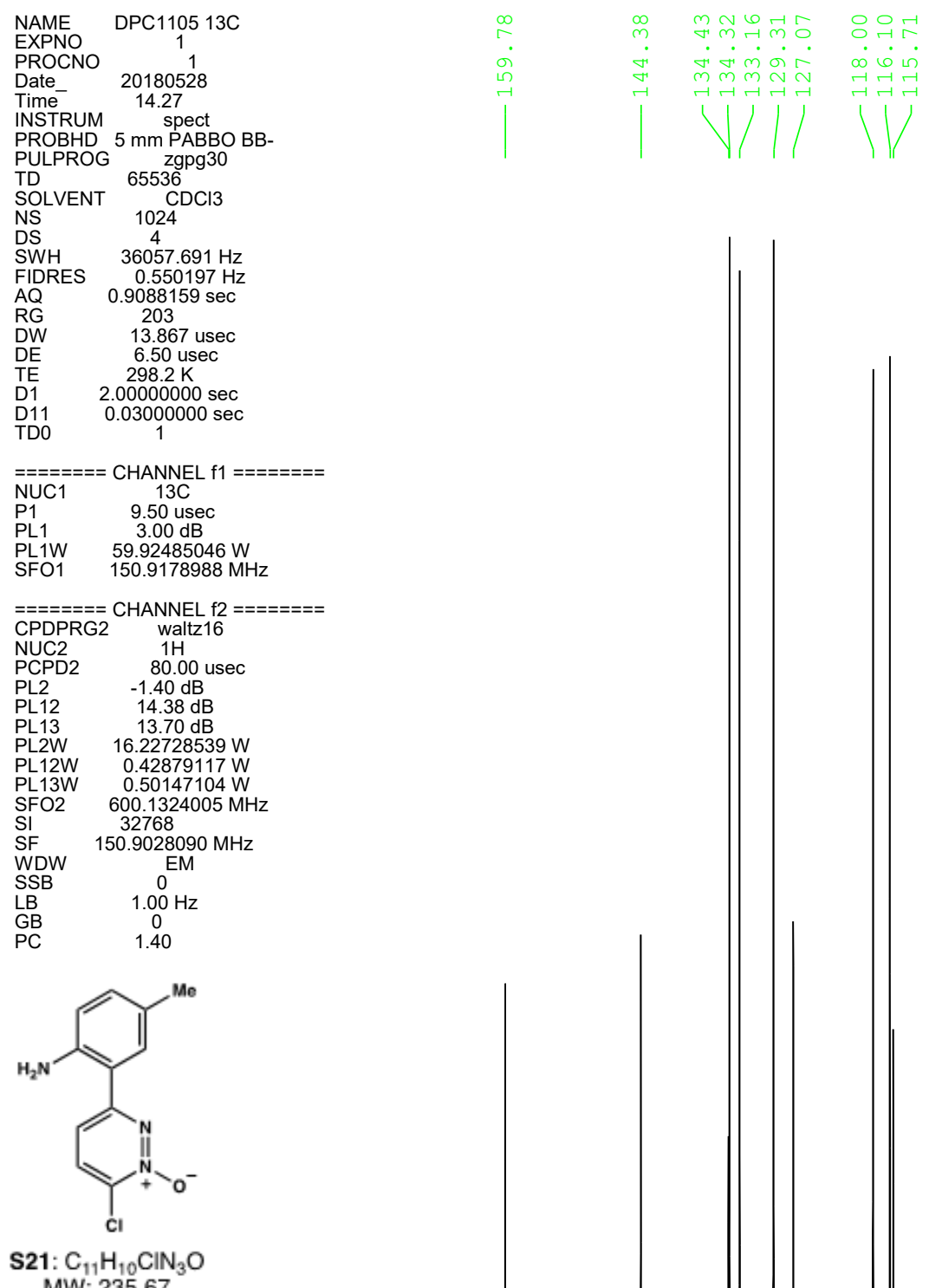

MW:

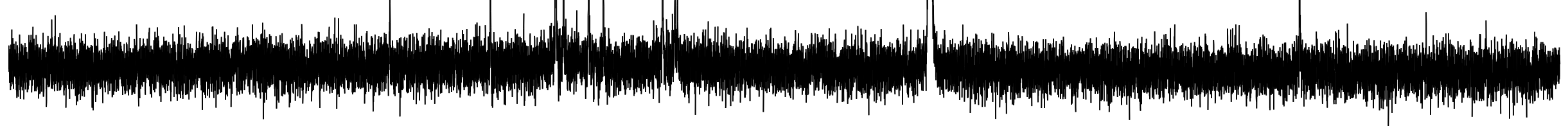



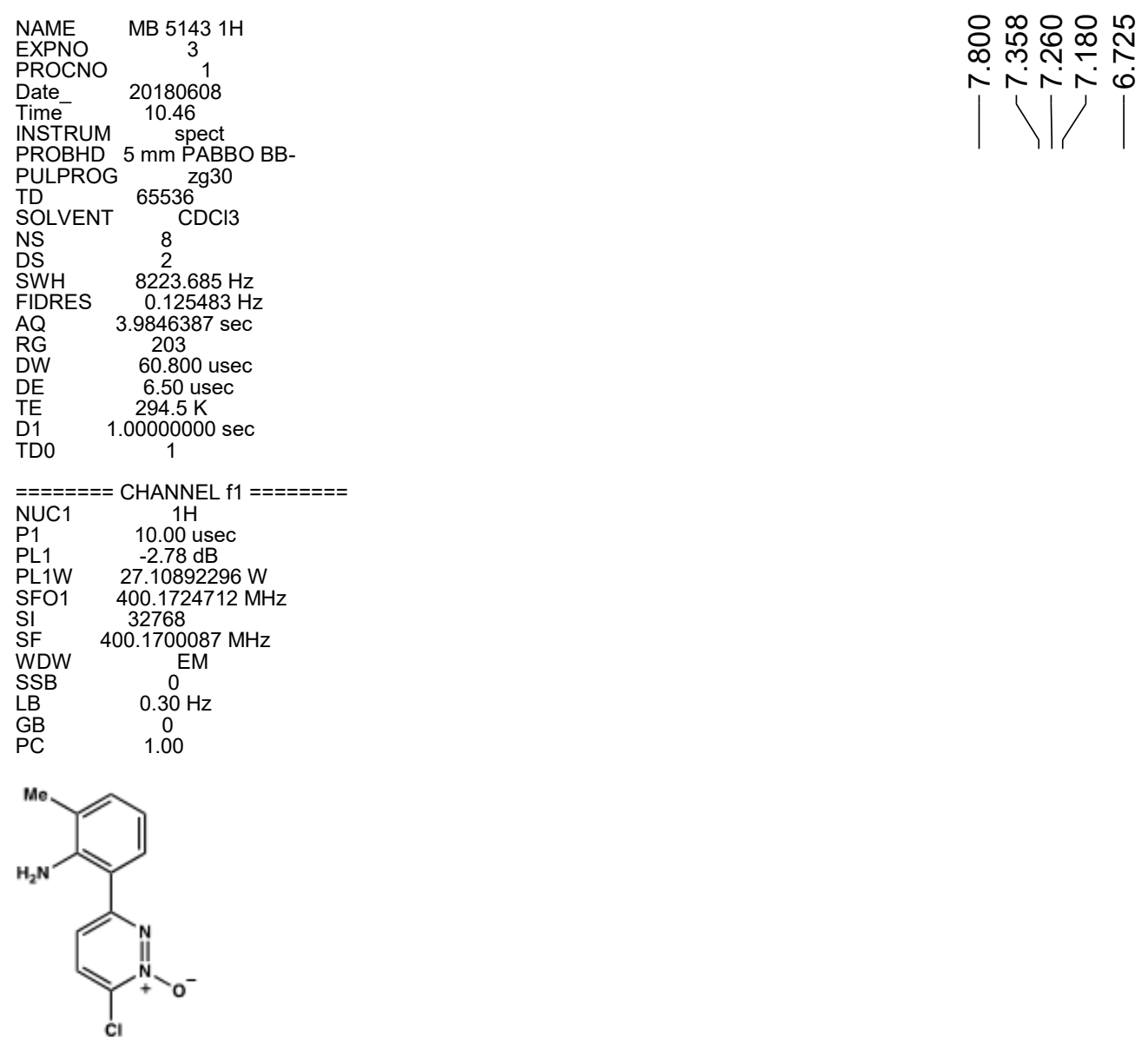

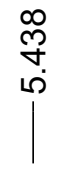

INSTRUM $5 \mathrm{spect}$

PULPROG $\quad$ zg30

SOLVENT CDCl3

DS

SWH $\quad 8223.685 \mathrm{~Hz}$

$3.12387 \mathrm{~Hz}$

203

DE $\quad 6.50$ usec

TD0 1.000000

$========$ CHANNEL $\mathrm{f} 1$ ========

SFO1 $\quad 400.1724712 \mathrm{MHz}$

SF $\quad 400.1700087 \mathrm{MHz}$

SSB $\quad 0$

$\begin{array}{ll}\mathrm{GB} & 0 \\ \mathrm{PC} & 1.00\end{array}$

S22: $\mathrm{C}_{11} \mathrm{H}_{10} \mathrm{ClN}_{3} \mathrm{O}$

MW: 235.67
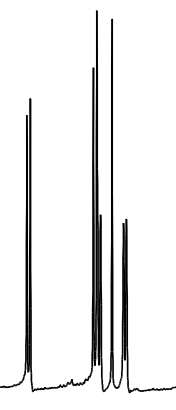

14

|ร. 7 


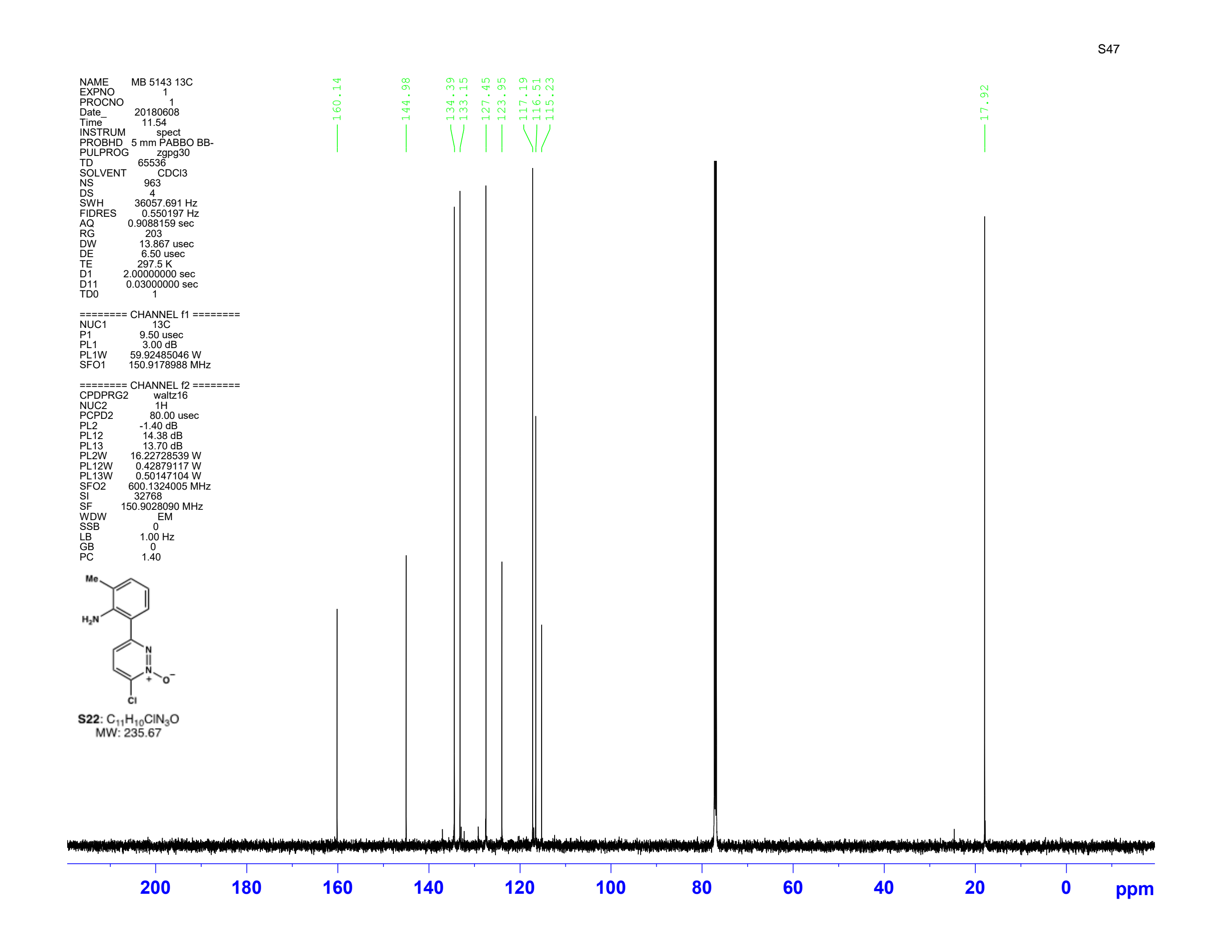




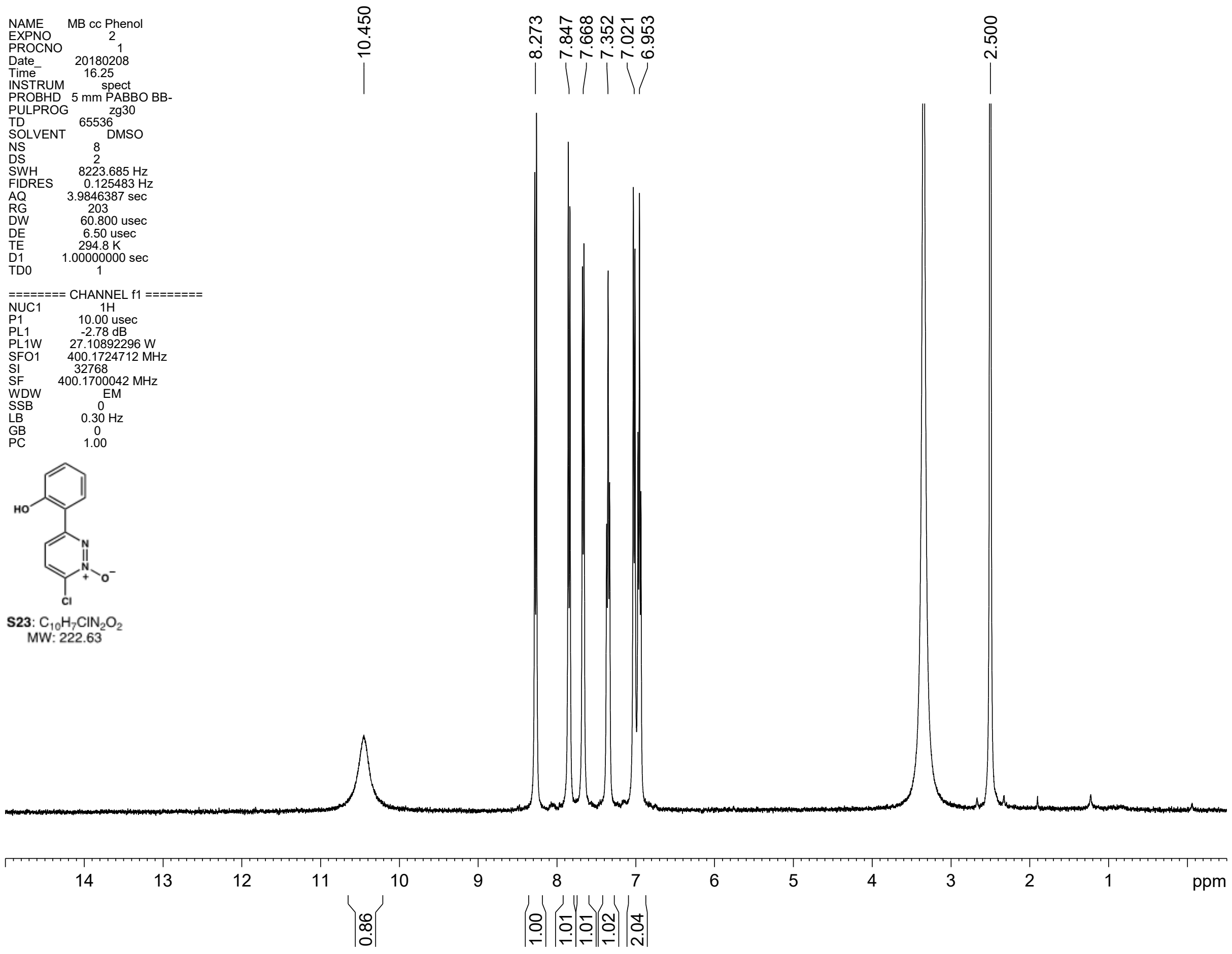



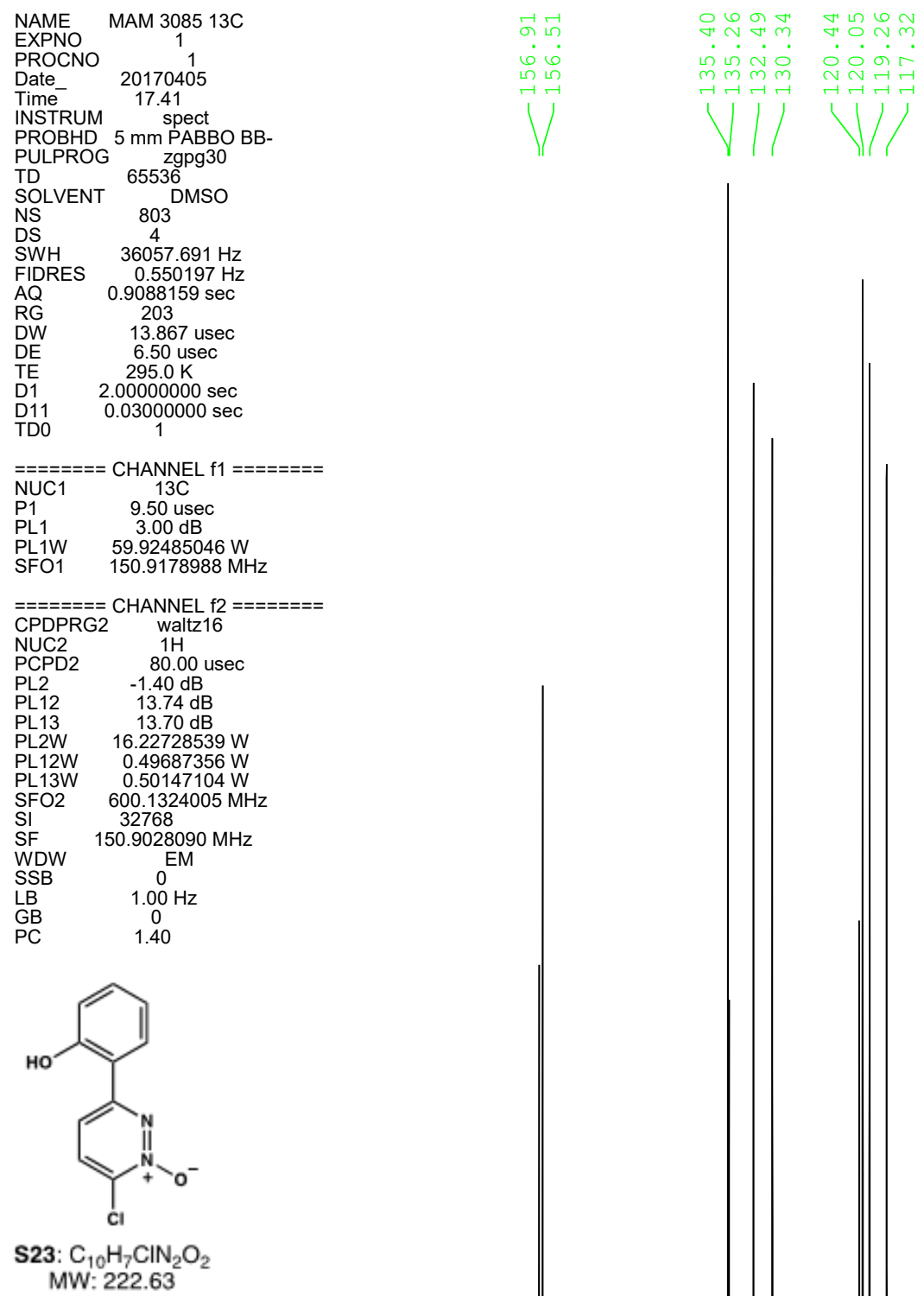

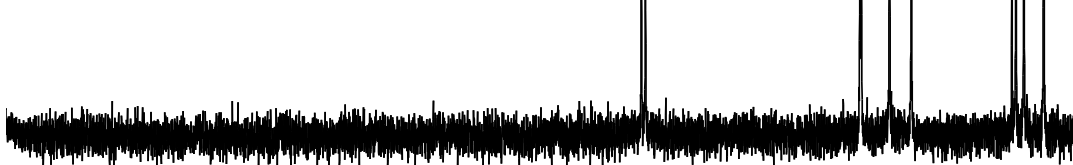

\begin{tabular}{rrrrrrrrrrr|r}
\hline 200 & 180 & 160 & 140 & 120 & 100 & 80 & 60 & 40 & 20 & 0 & ppm
\end{tabular}




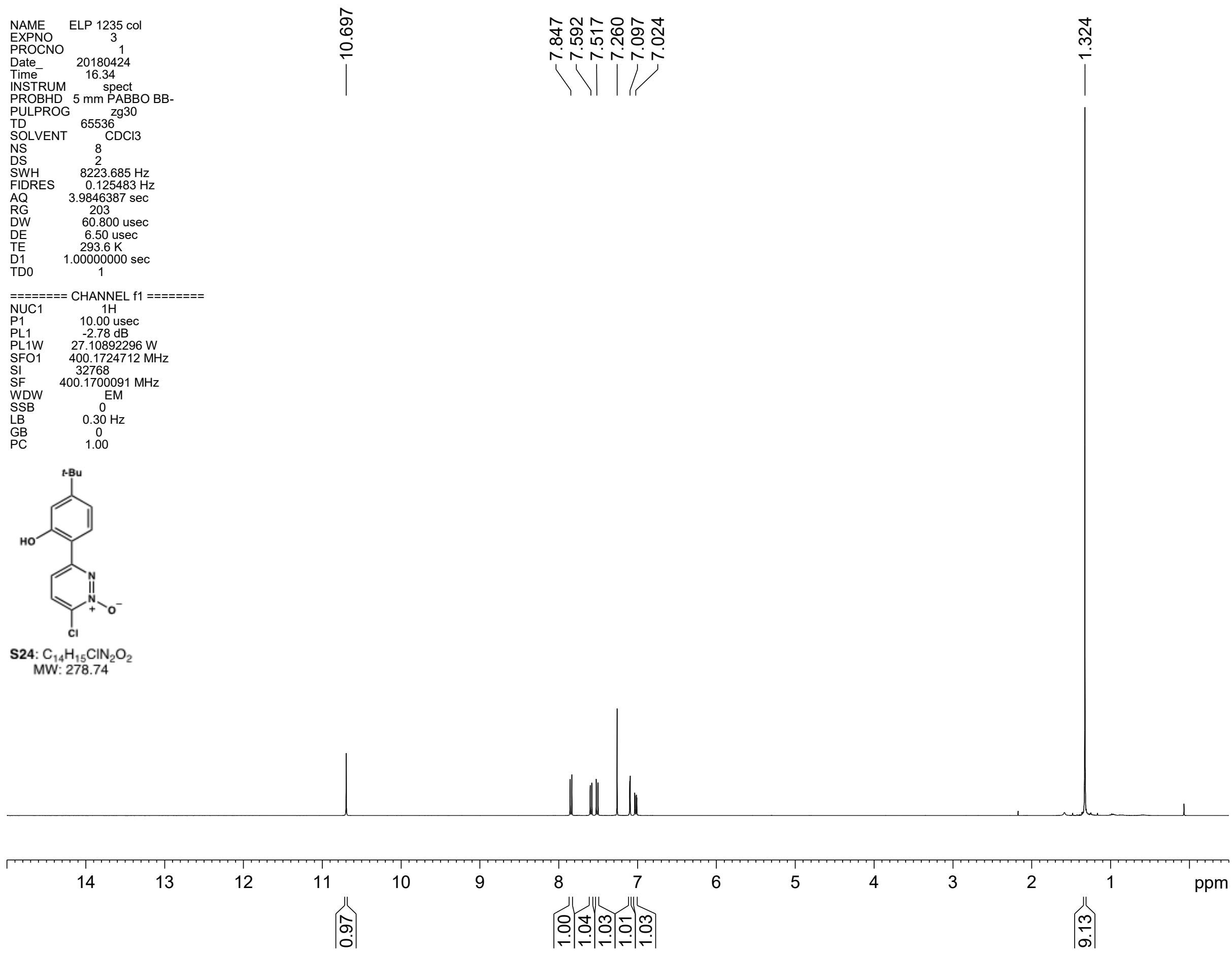




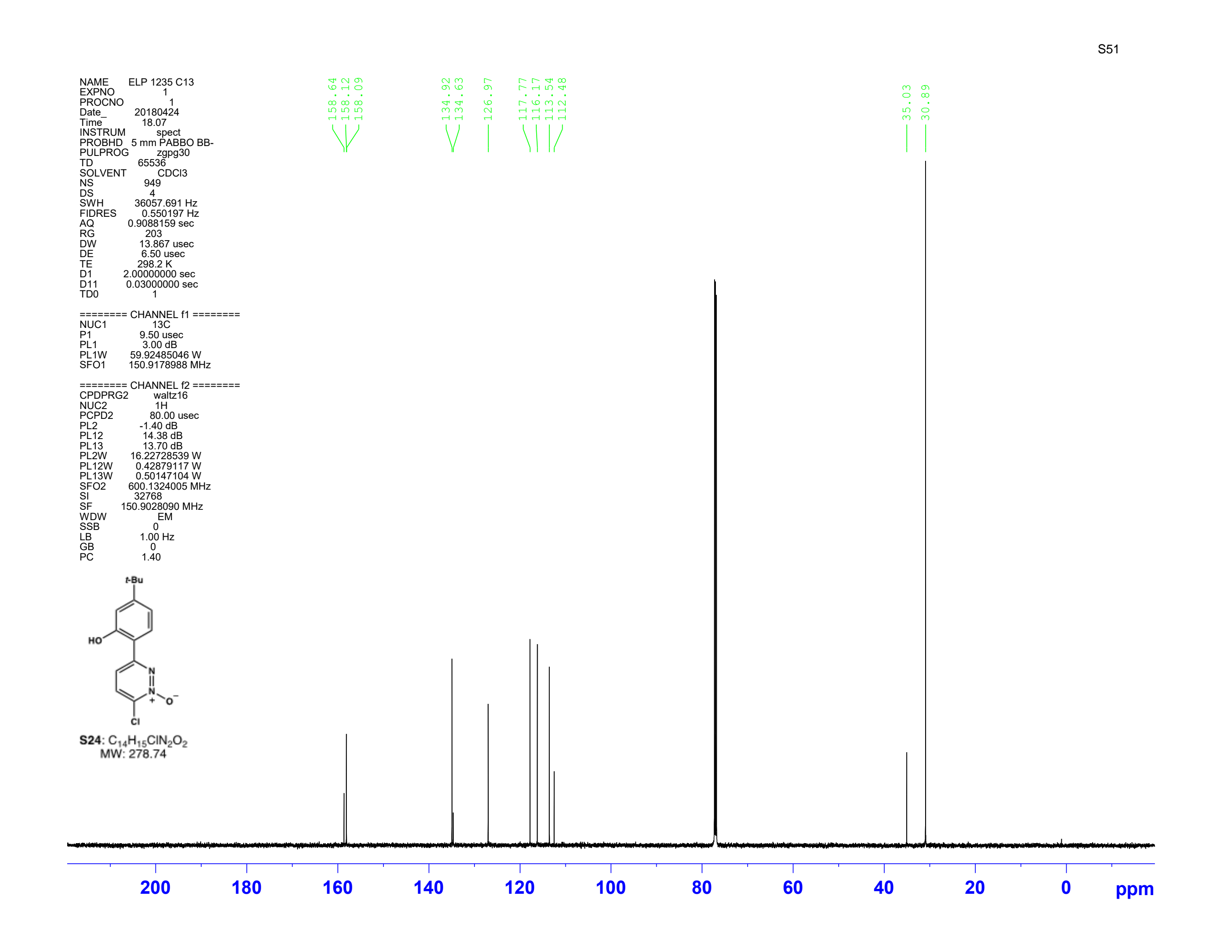



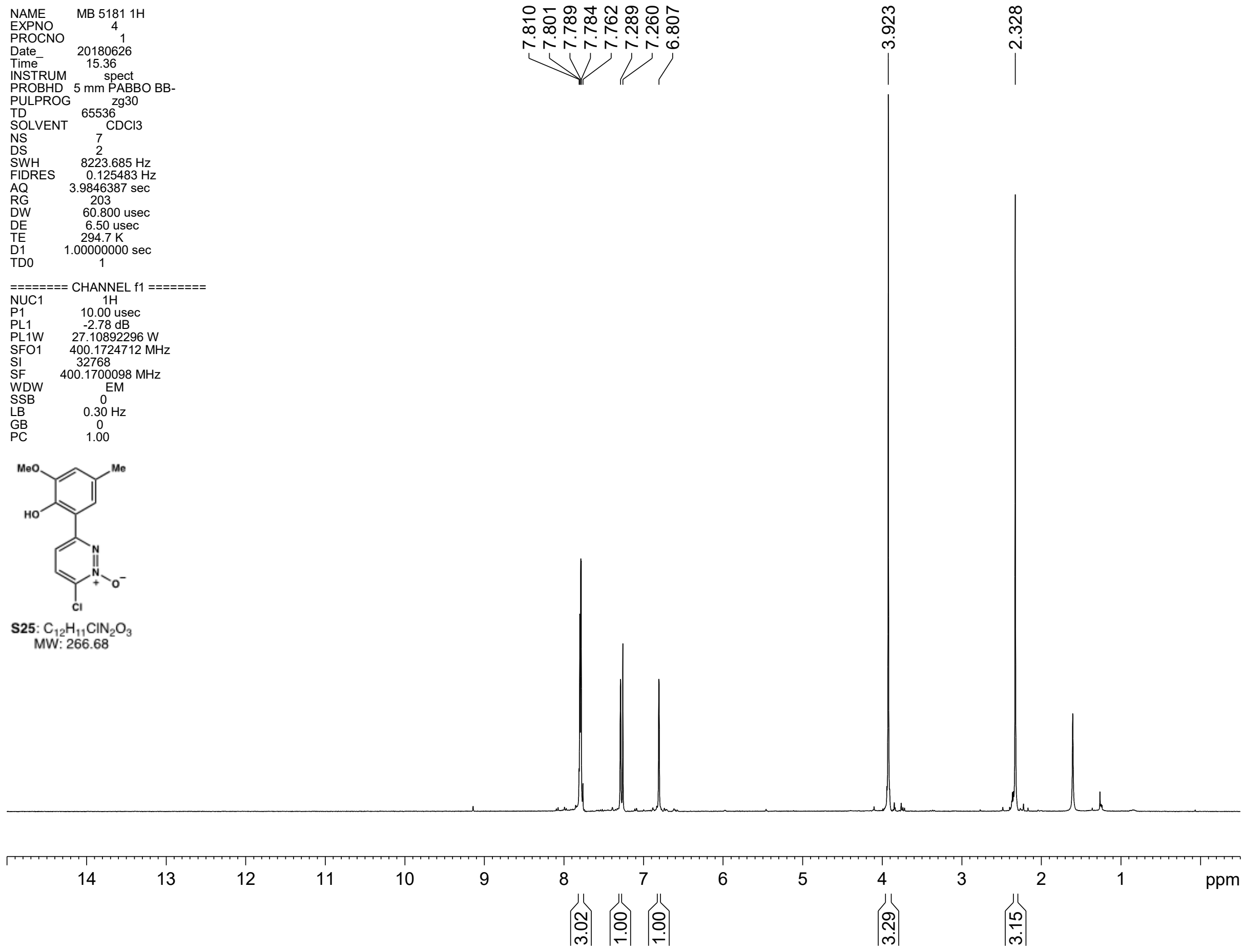


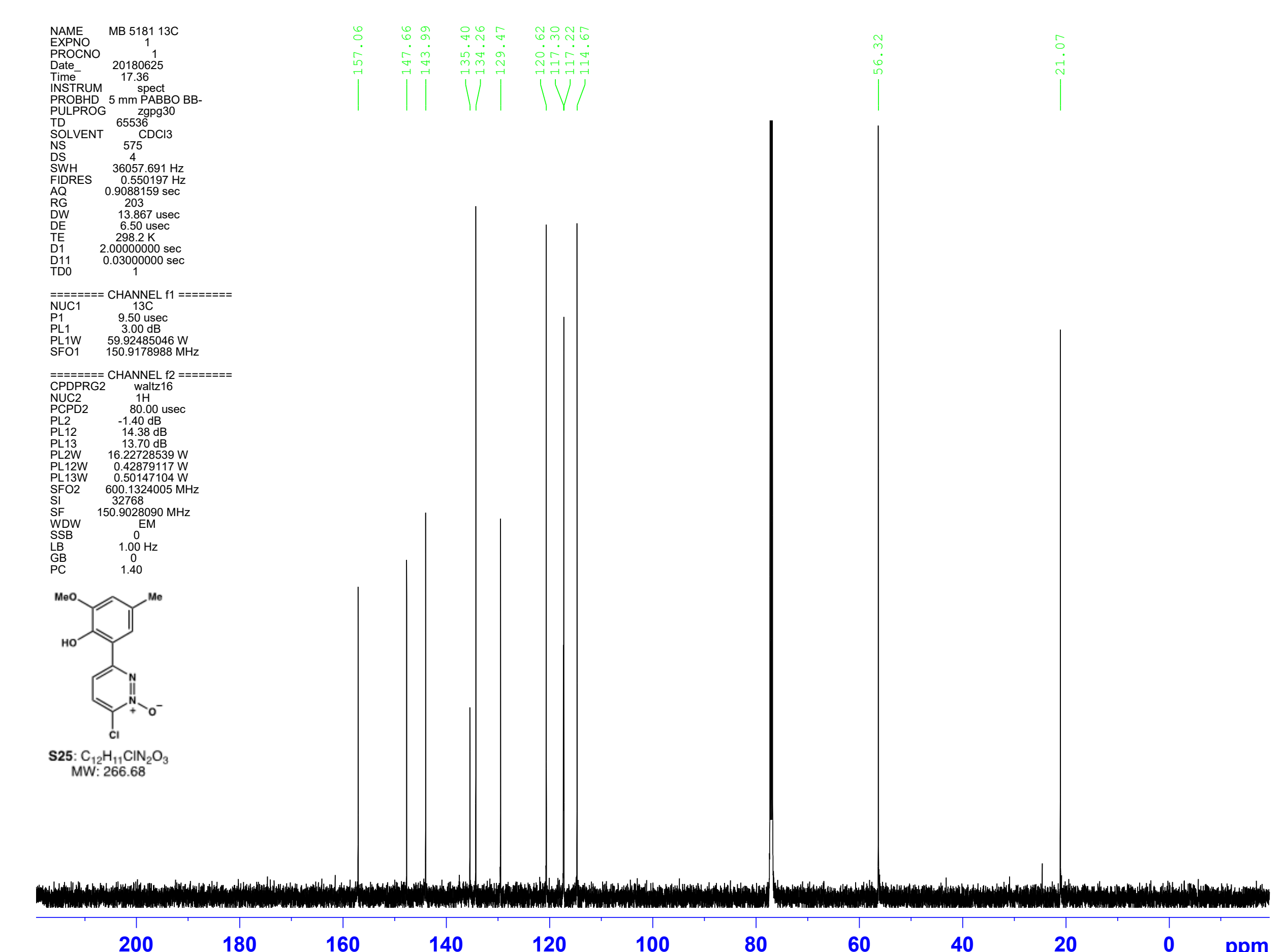




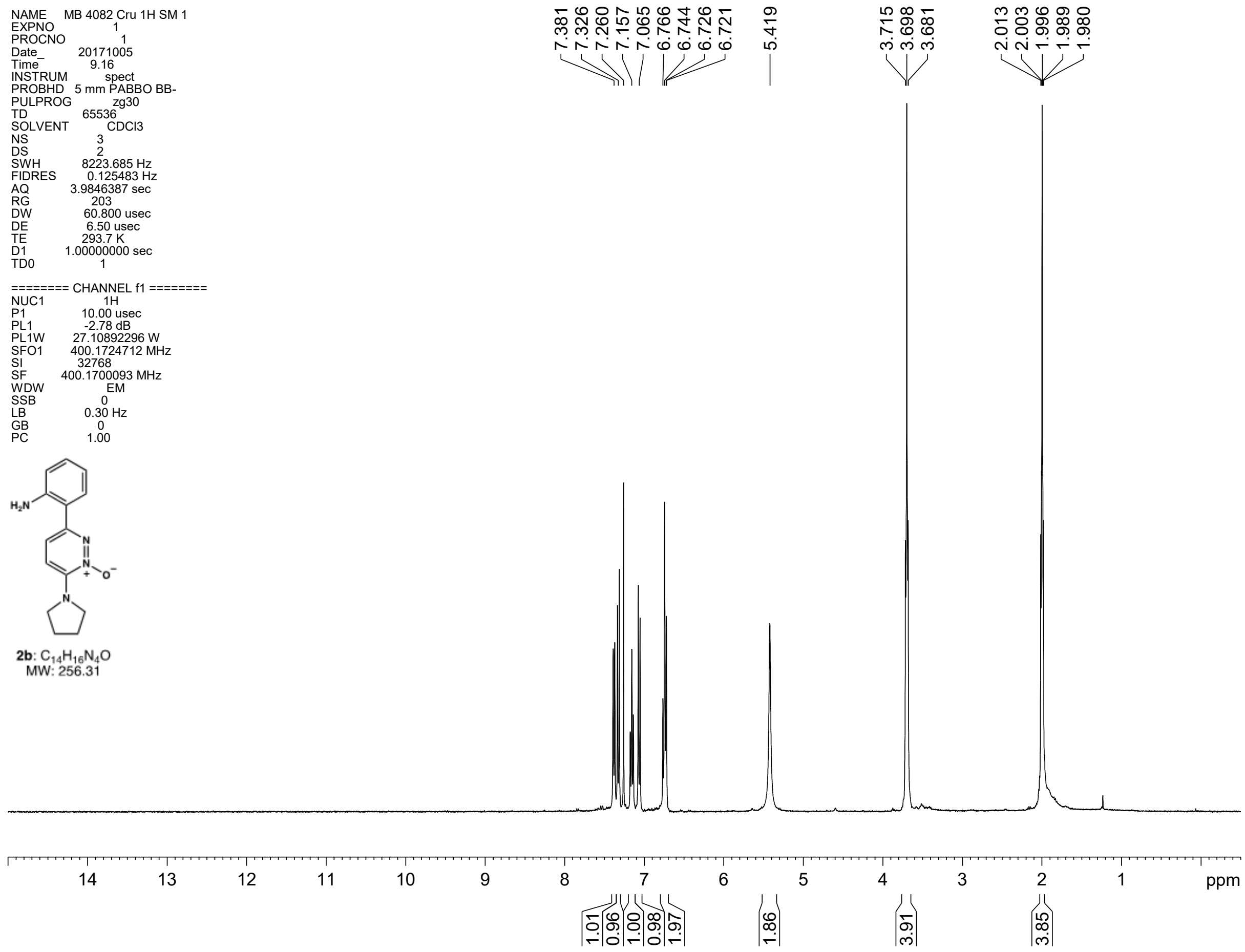




$$
\frac{1}{1}
$$




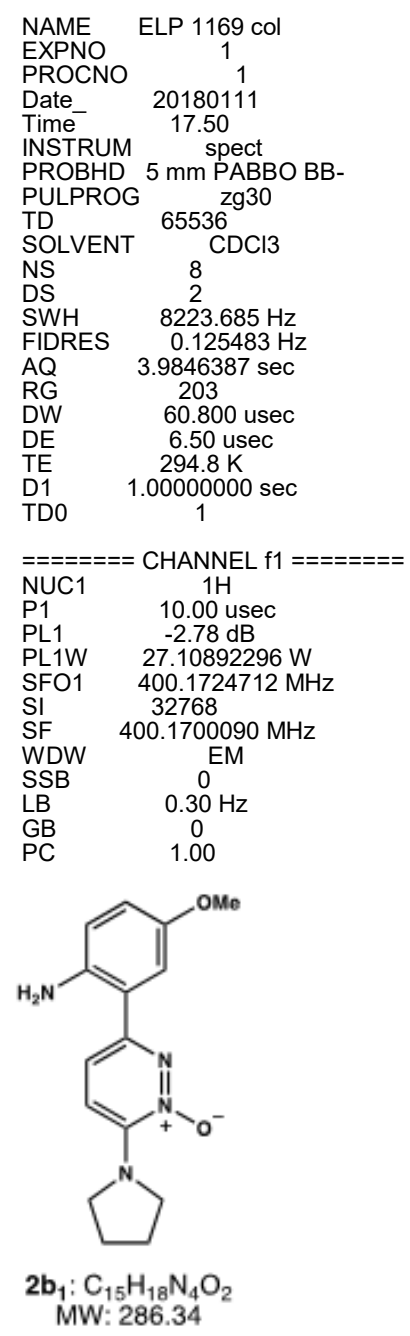

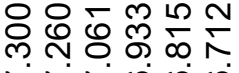

노요요

लिंलि

ก ก

สิธิ์

mm

Nij

PROBHD $5 \mathrm{~mm}$ PABBO BB-

PULPROG $\quad \mathrm{zg} 30$

$0.12583 \mathrm{~Hz}$

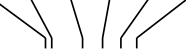

$1 /$

$M W:$
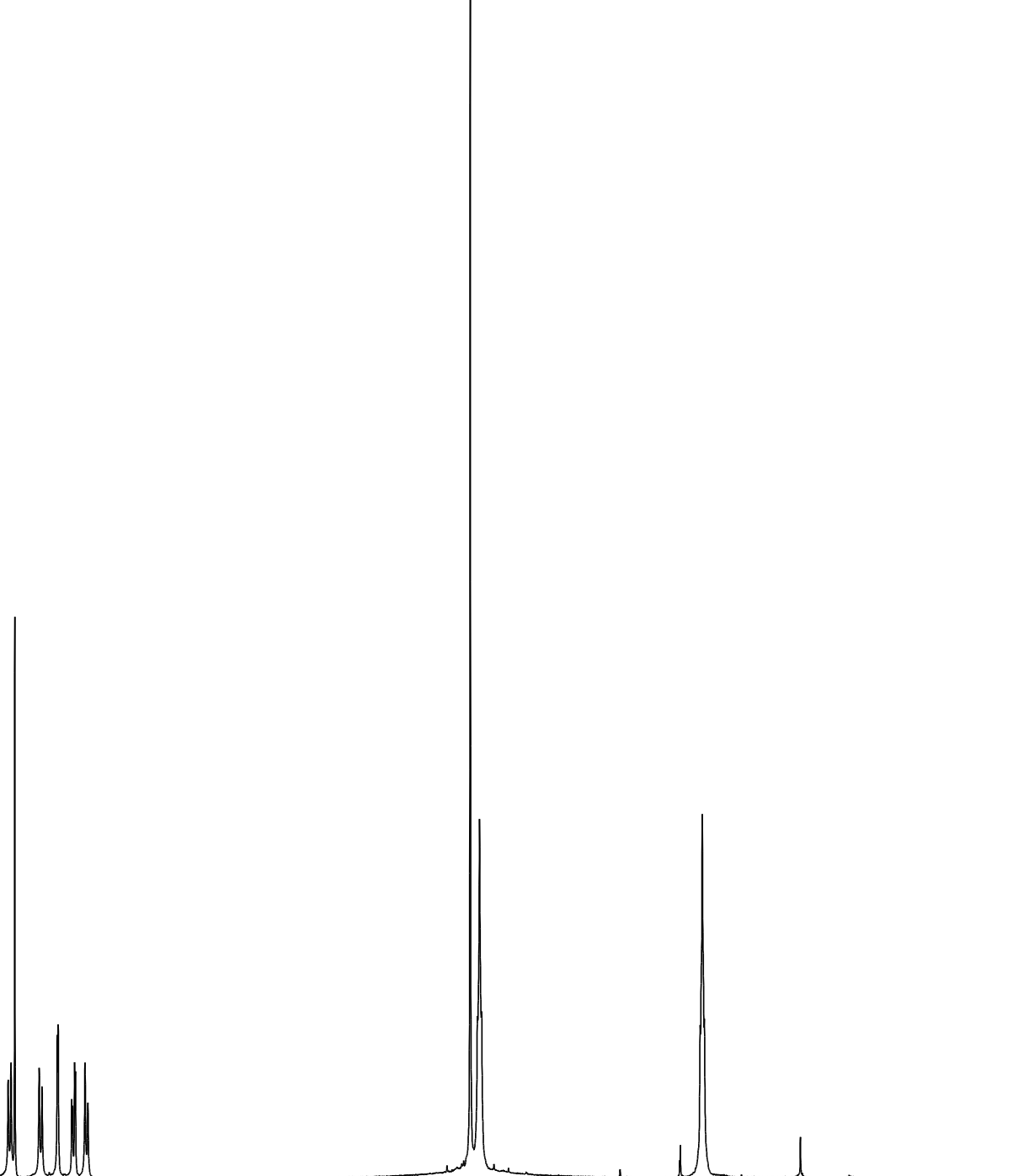

14

9




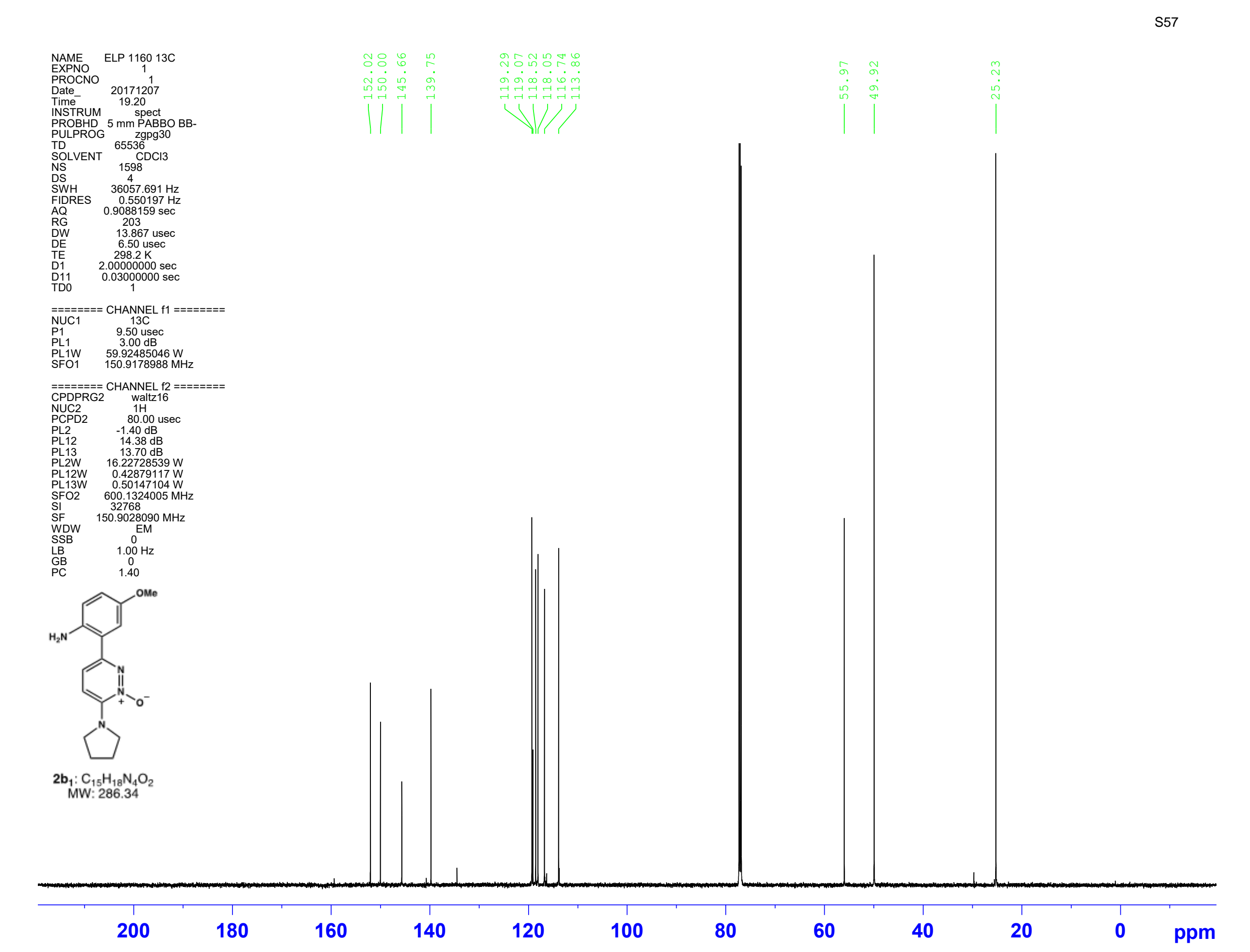



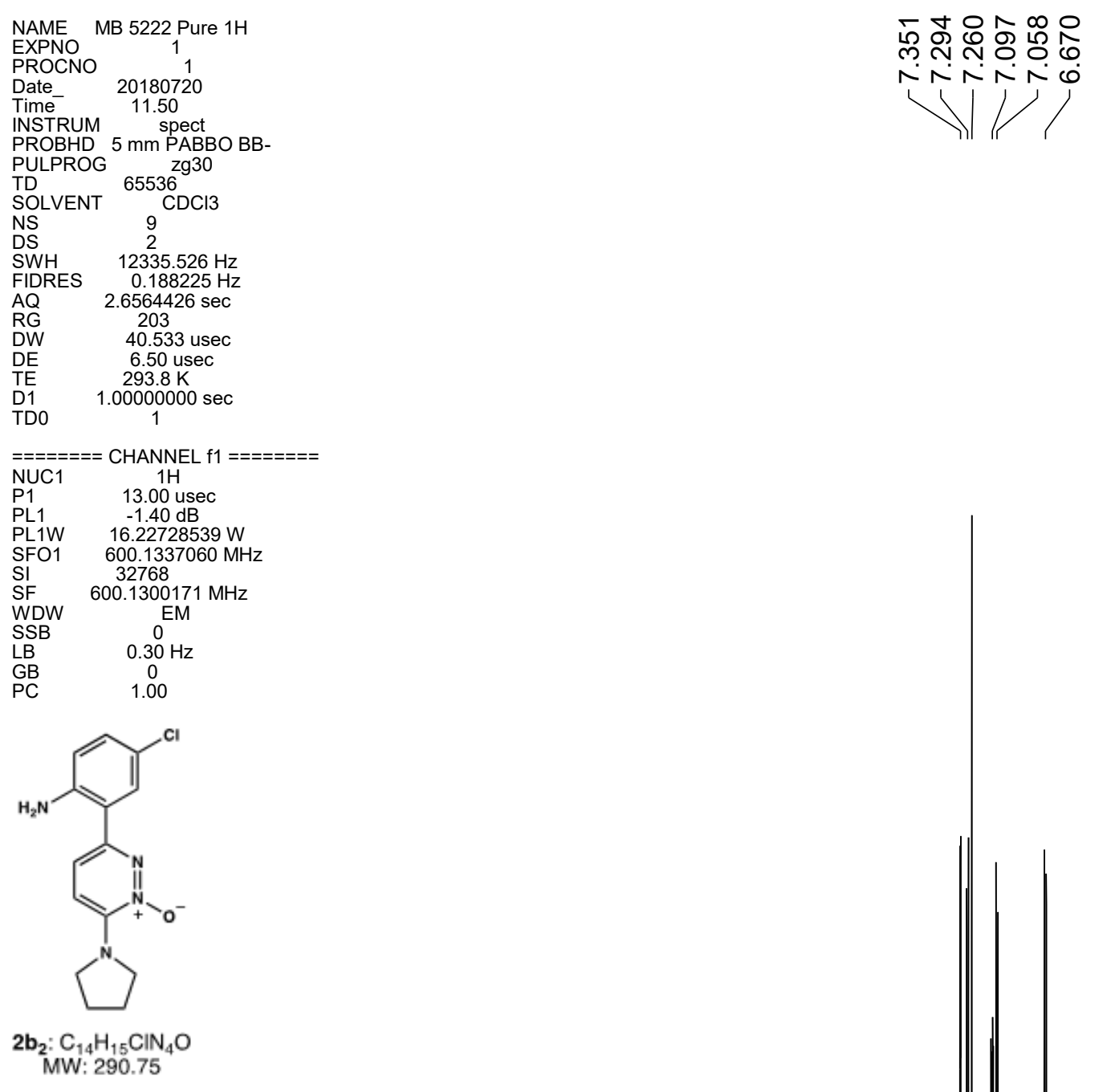

ㅇํำ

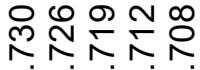

은ํํㅇํ요

लं $\dot{m} \dot{m}$

N่ N

1

PROBHD $5 \mathrm{~mm}$ PABBO BB

PULPROG $\mathrm{zg} 30$

SWH

$=======$ CHANNEL $\mathrm{f} 1$ ========

$\mathrm{PC}$

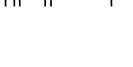

11
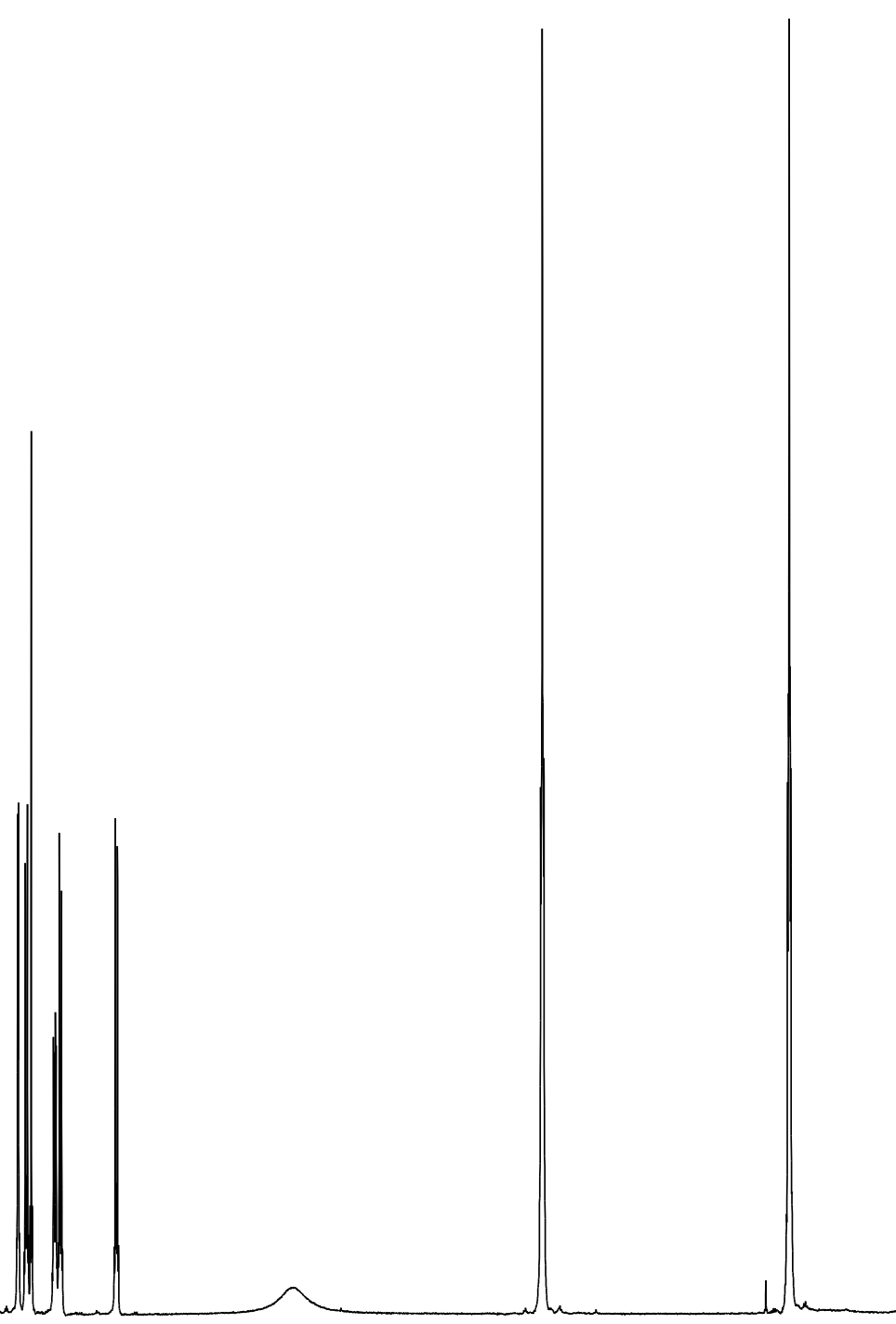

13

12

11

9

8

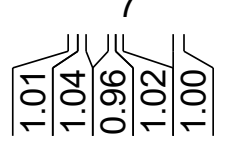

$\underbrace{6}$

5

4
0
0
8

3

$\left|\begin{array}{l}2 \\ 0 \\ \frac{0}{8}\end{array}\right|$

$\left|\begin{array}{l}\infty \\ 0 \\ \dot{j}\end{array}\right|$ 

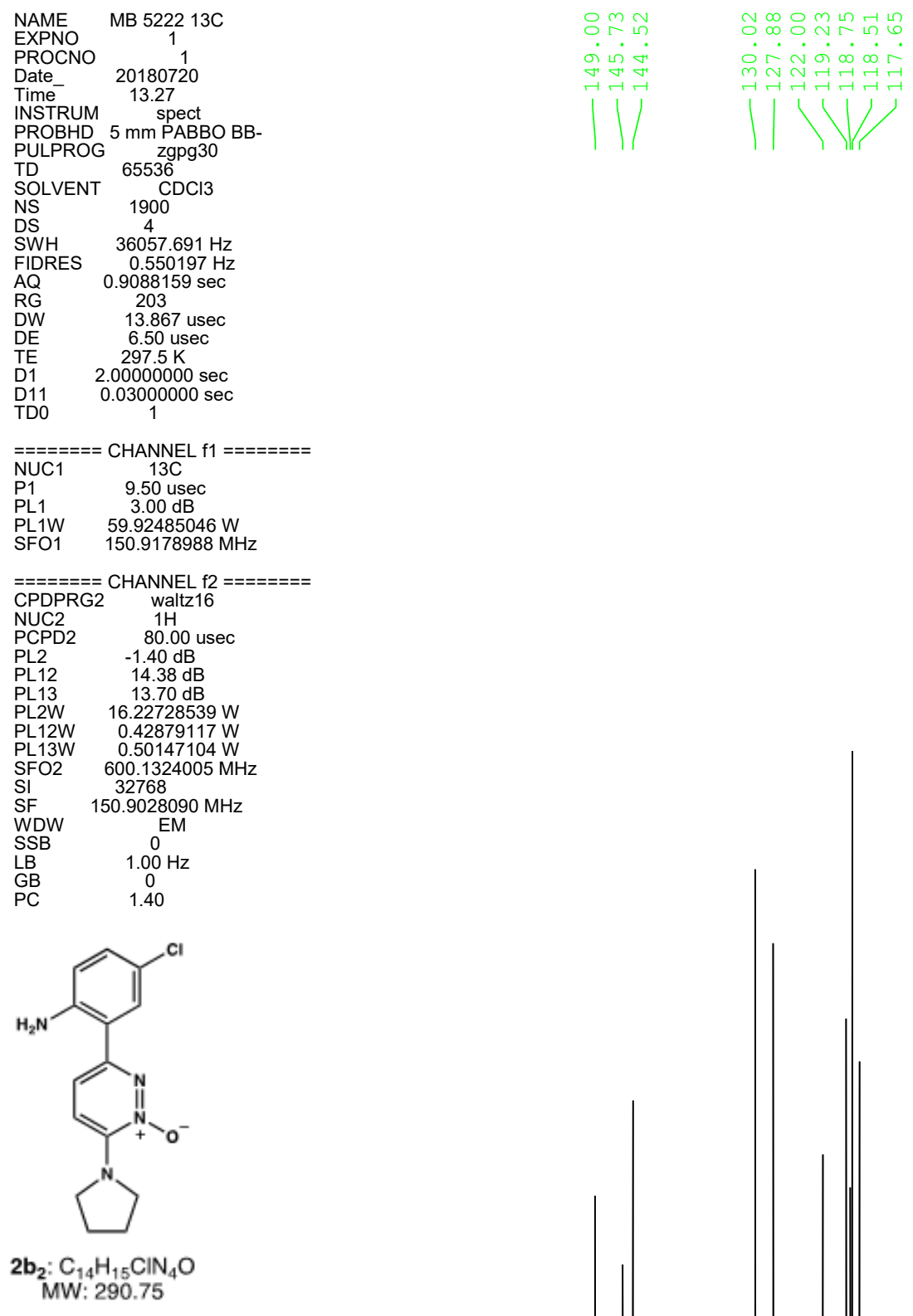

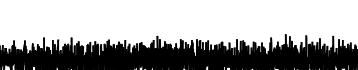

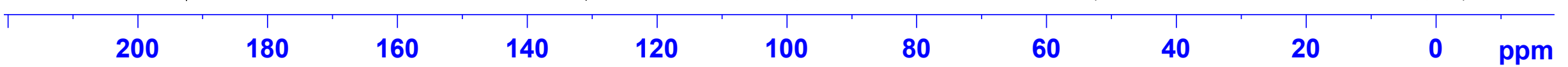



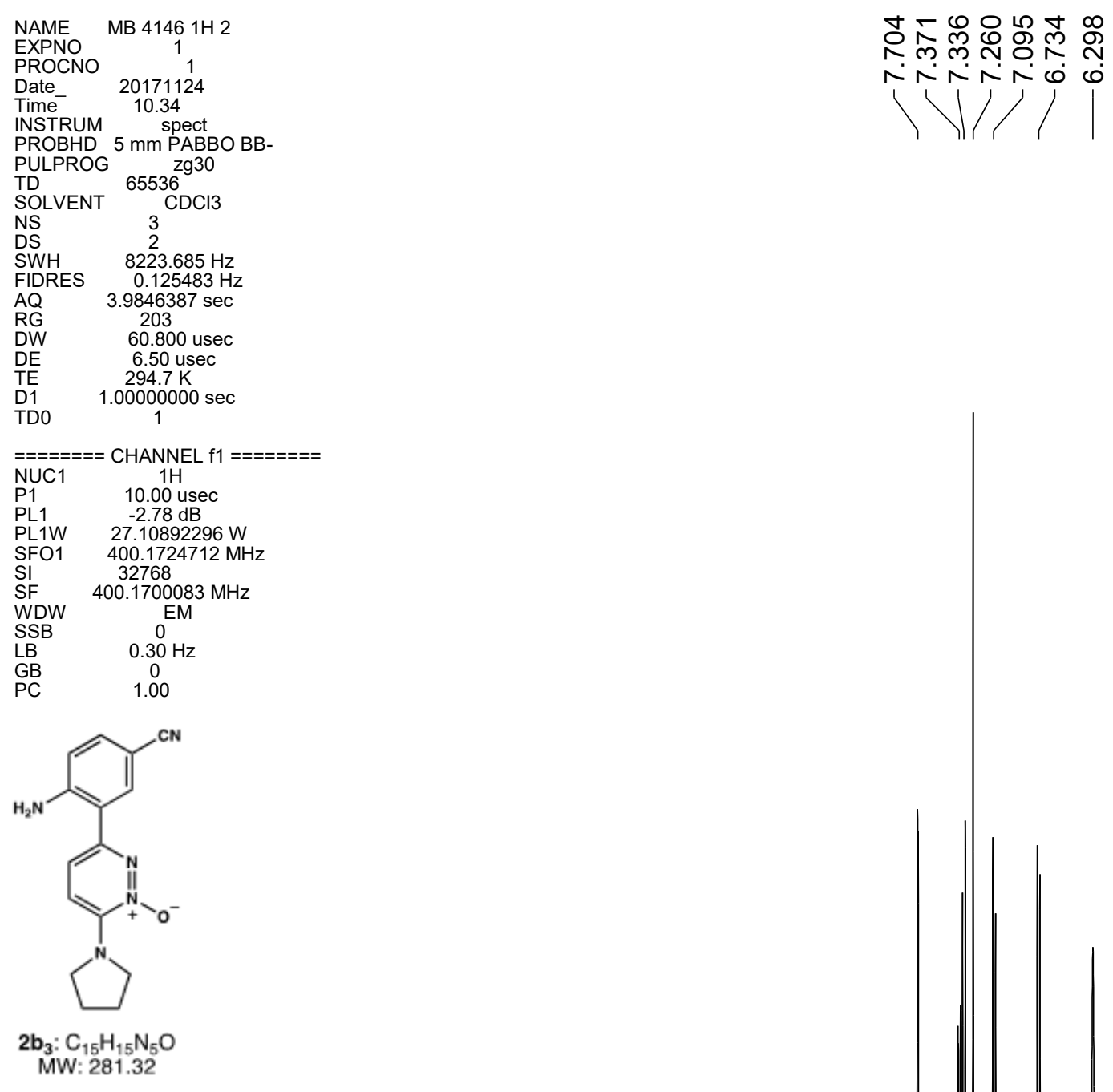

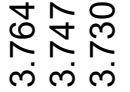

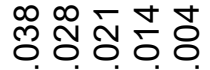

cं

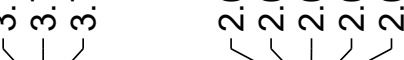

PROBHD 5 mm PABBO BB-

M//

$=======$ CHANNEL $\mathrm{f} 1=======$

SI $\quad 32768$

WDW $\quad$ EM

$\mathrm{LB} \quad 0.30 \mathrm{~Hz}$
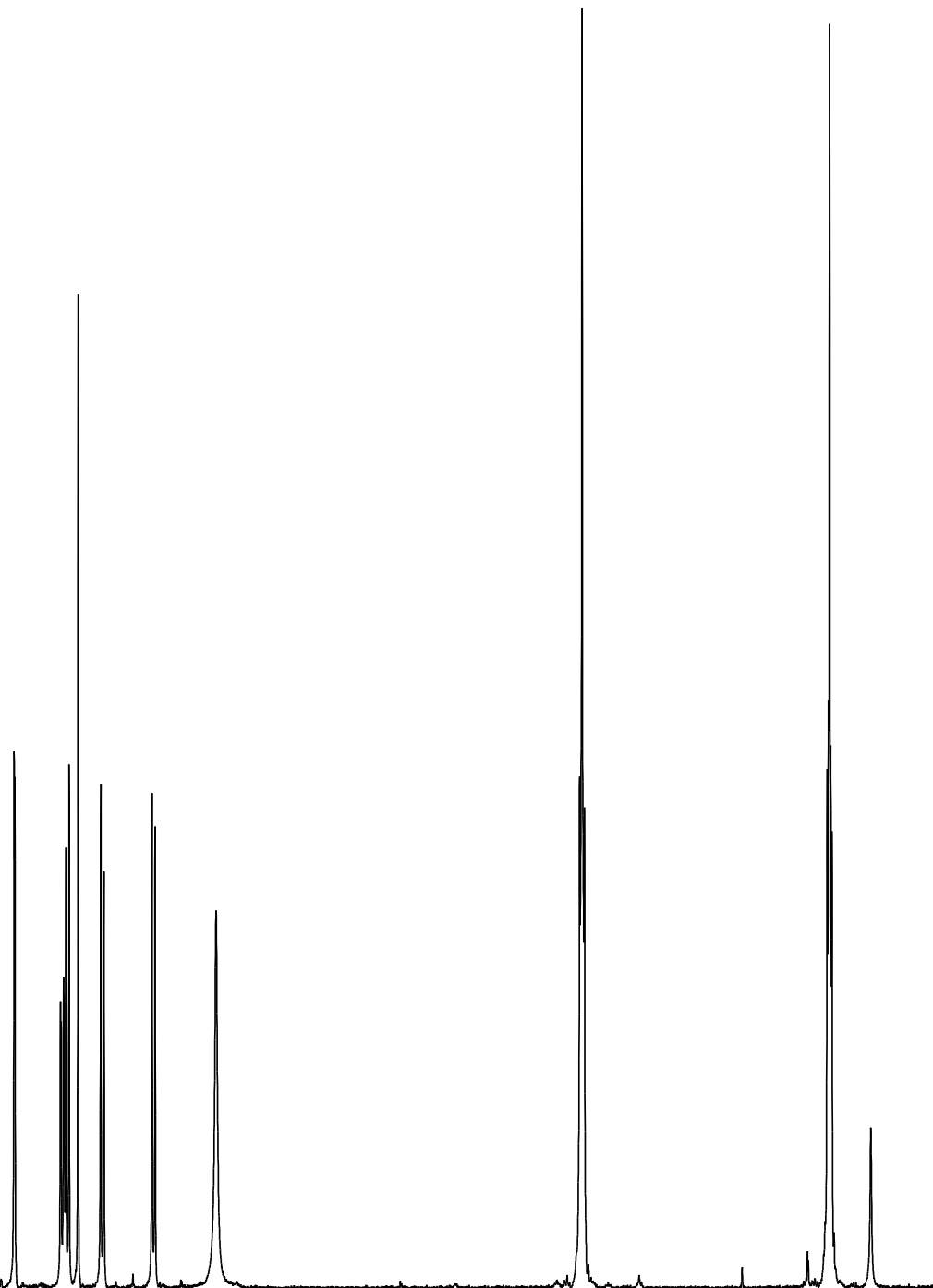

14

13

12

11

10

9

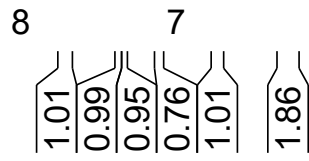

6

4

|人

3

2

1

$\left|\begin{array}{l}8 \\ \hdashline\end{array}\right|$ 


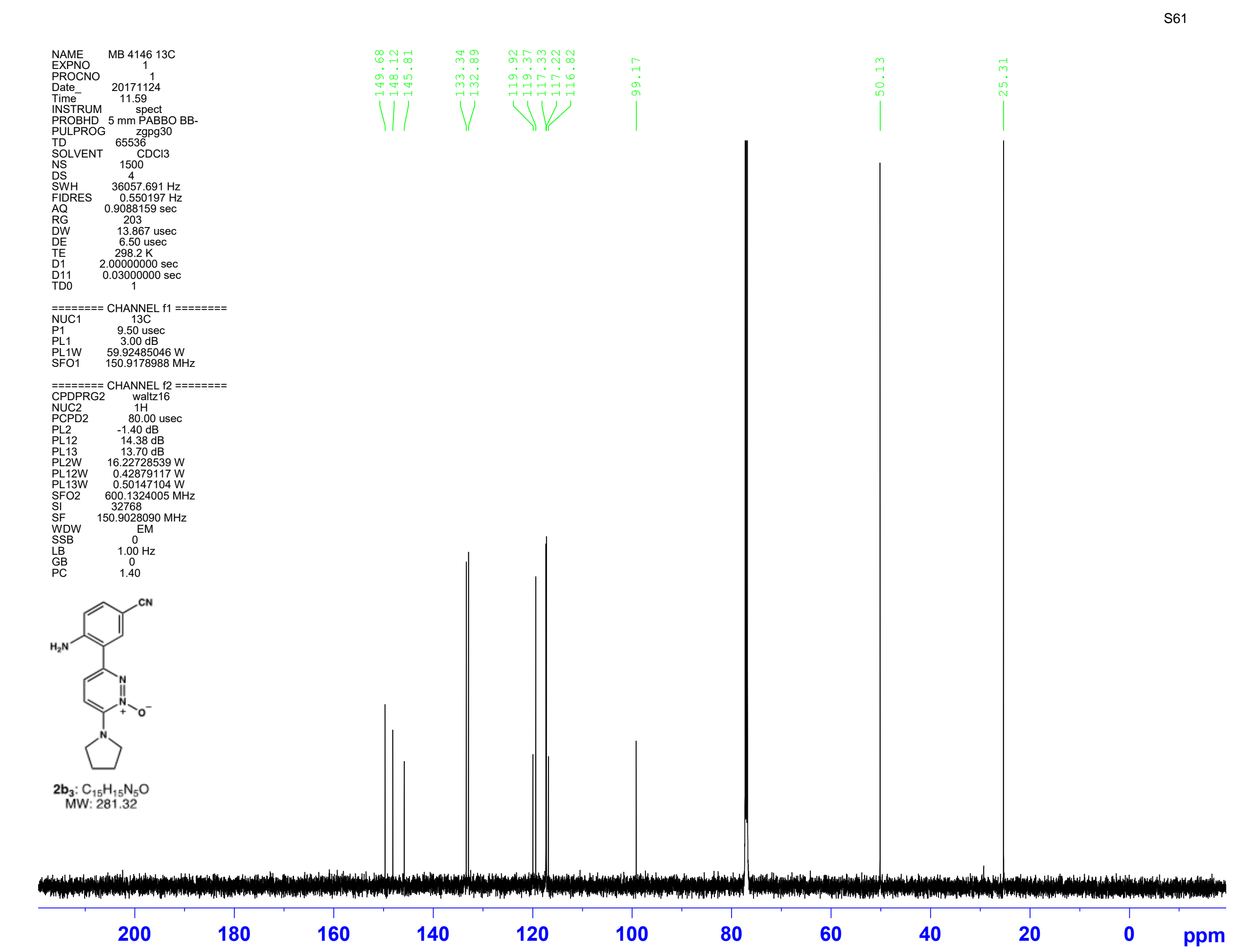



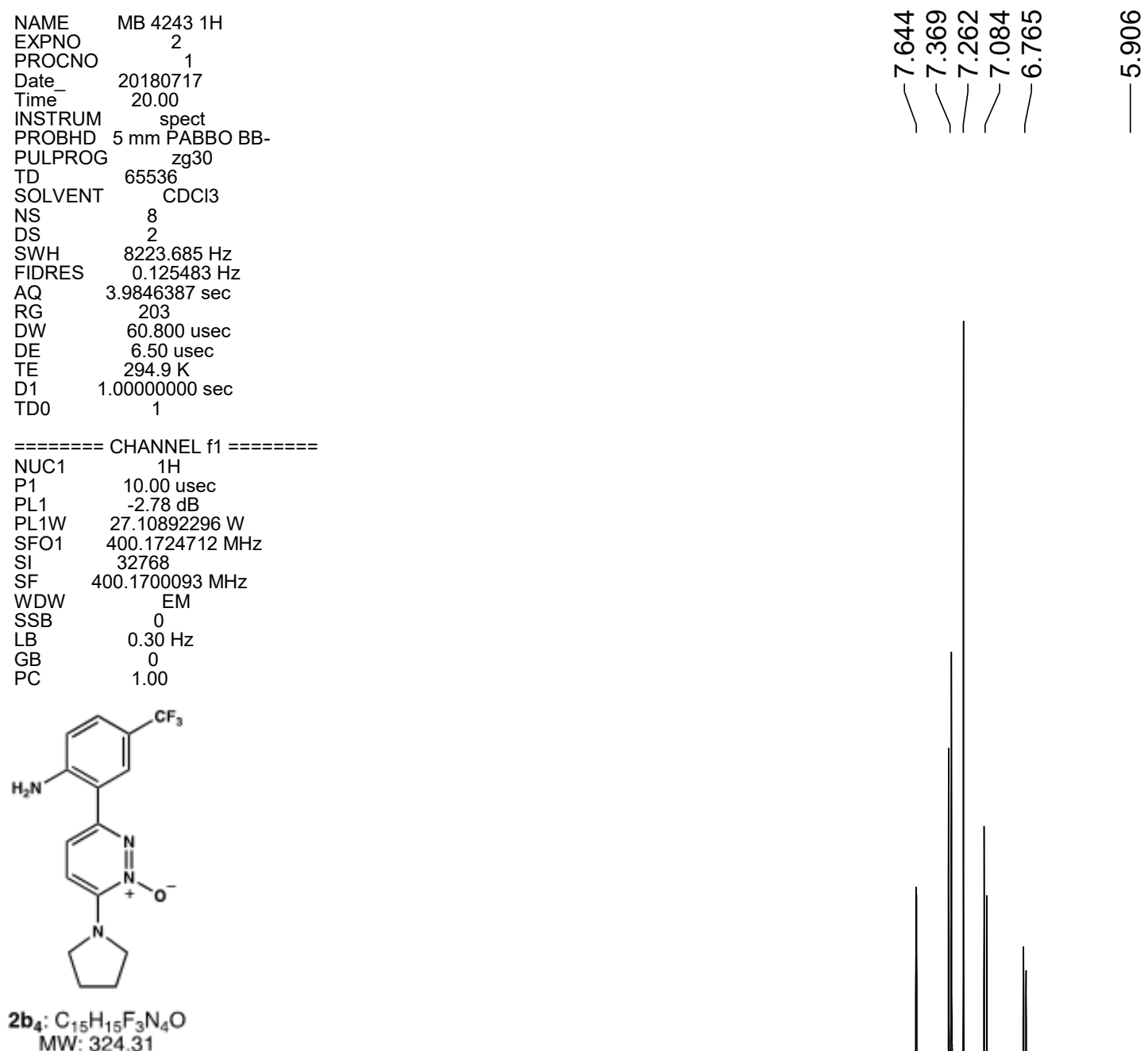

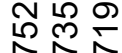

लंलें

PROBHD 5 mm PABBO BB-

PULPROG $\quad$ Zg 30

SOLVENT CDCl3

DS

SWH $\quad 8223.685 \mathrm{~Hz}$

$30.12583 \mathrm{~Hz}$

203

$=======$ CHANNEL $f 1$ =======

$\begin{array}{ll}S I & 32768\end{array}$

WDW EM

LB

$30 \mathrm{~Hz}$

MW: 324.31
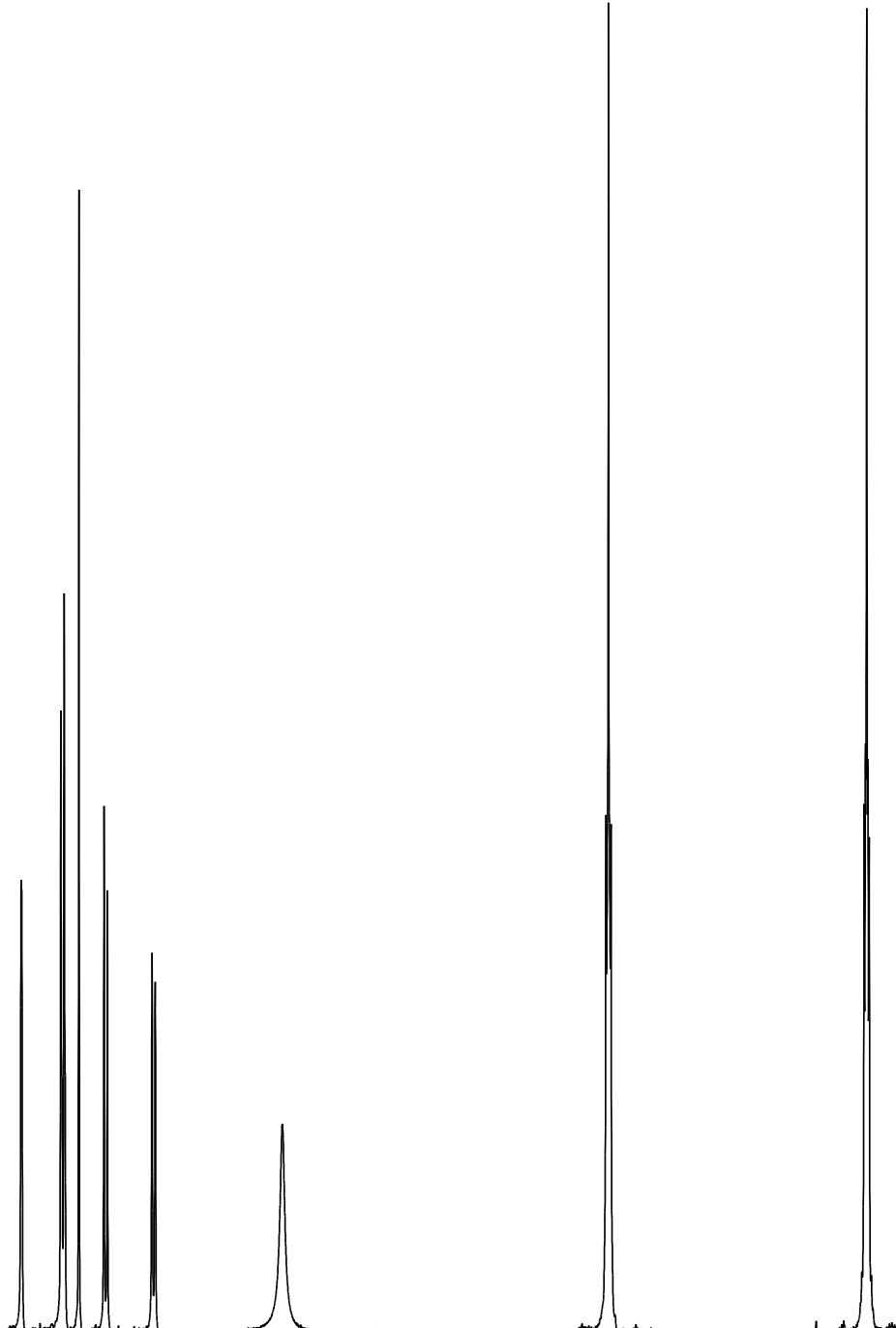

4
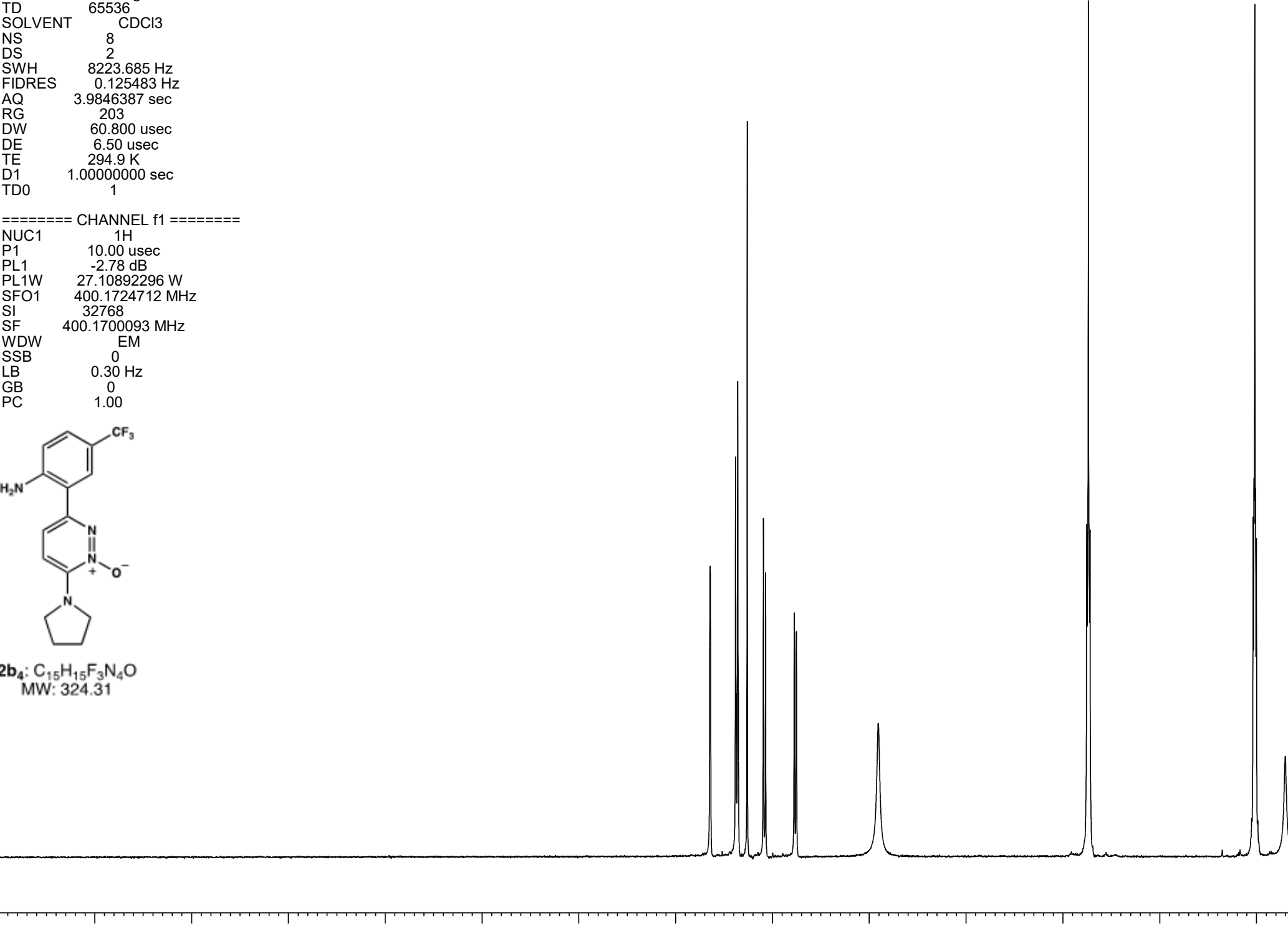

14

13

12

11

10

9

8

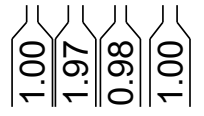

$\left|\begin{array}{c}\hat{\infty} \\ \dot{\infty}\end{array}\right|$

5

4

$\left|\begin{array}{l}\infty \\ \infty \\ m\end{array}\right|$

3

2

命 



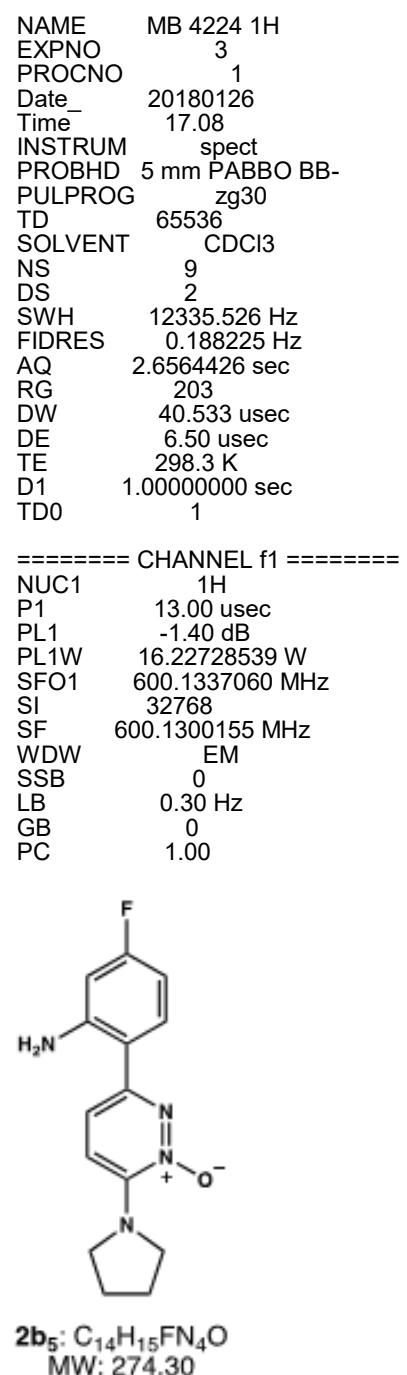

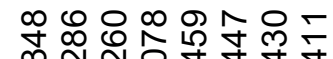
- N

PROBHD 5 mmPABBO BB-
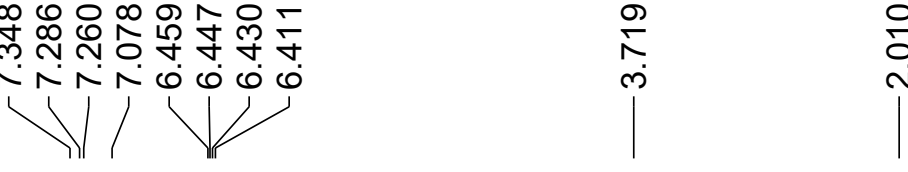

MW: 274.30
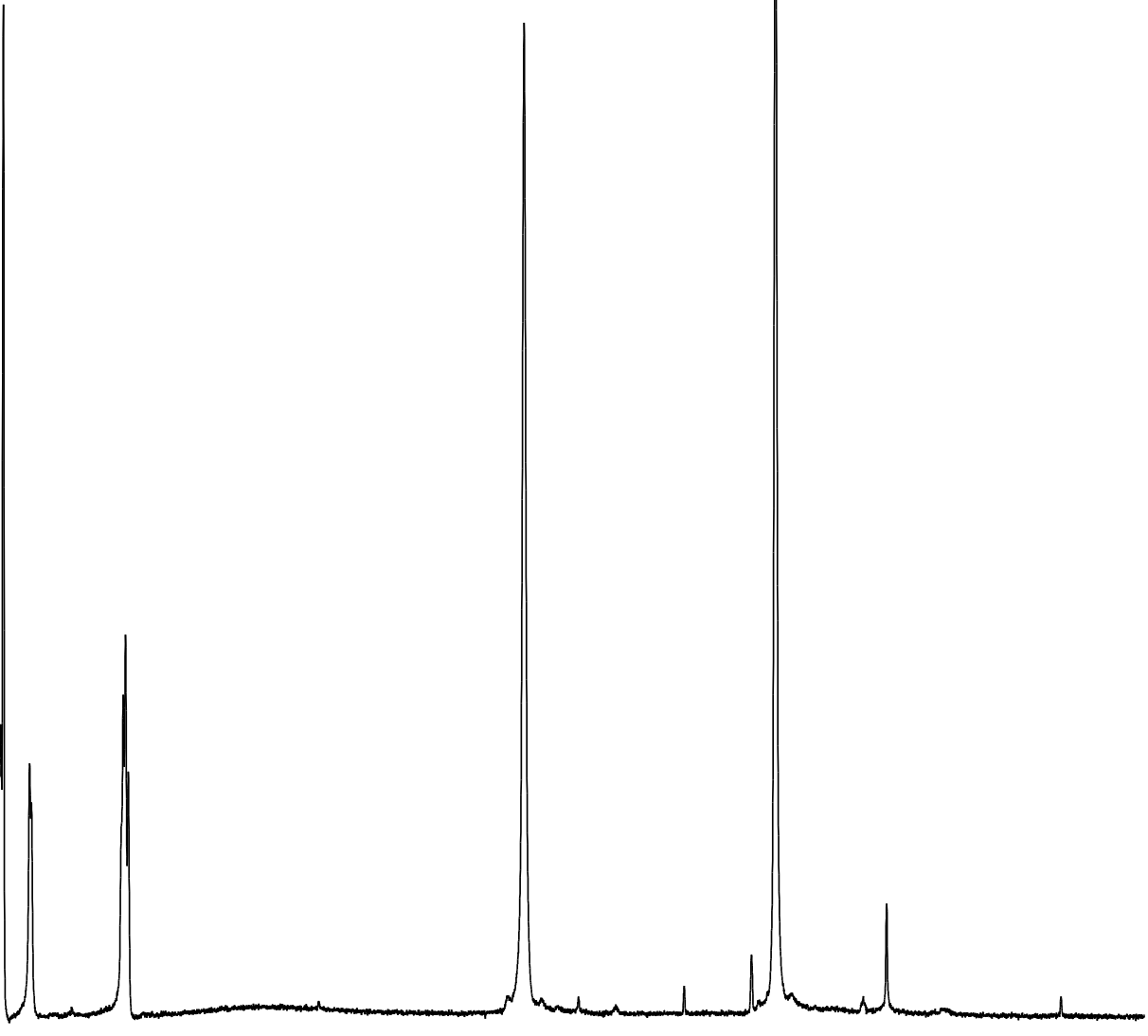

14

13

12

11

10

9

8

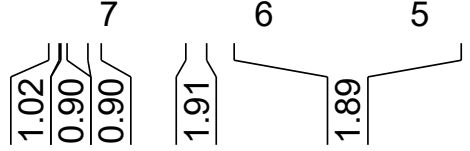

$\left|\begin{array}{l}0 \\ \infty \\ m\end{array}\right|$

$\left|\begin{array}{c}2 \\ 8 \\ 8 \\ \dot{\gamma}\end{array}\right|$ 

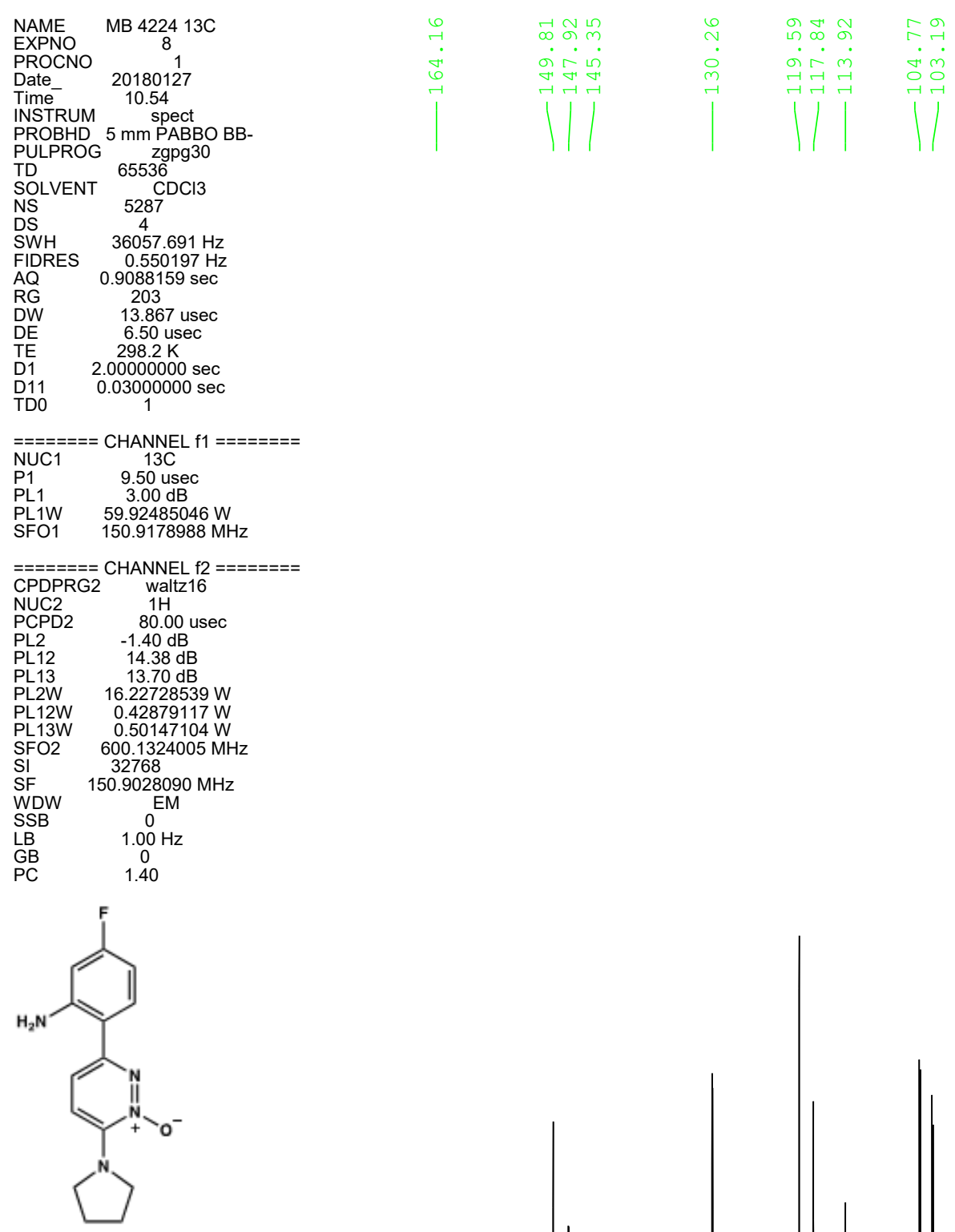

$2 b_{5}: \mathrm{C}_{14} \mathrm{H}_{15} \mathrm{FN}_{4} \mathrm{O}$ MW: 274.30

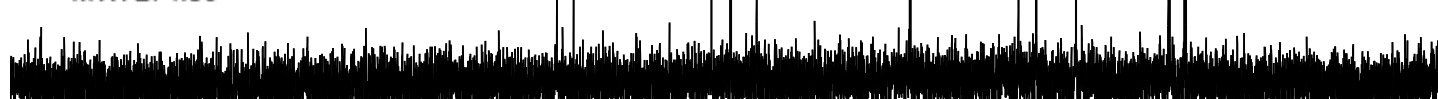

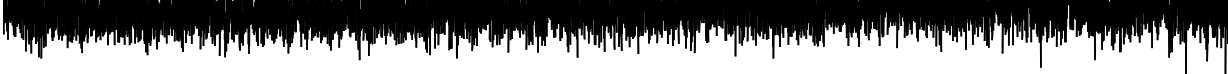

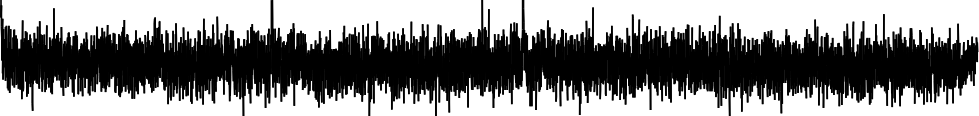




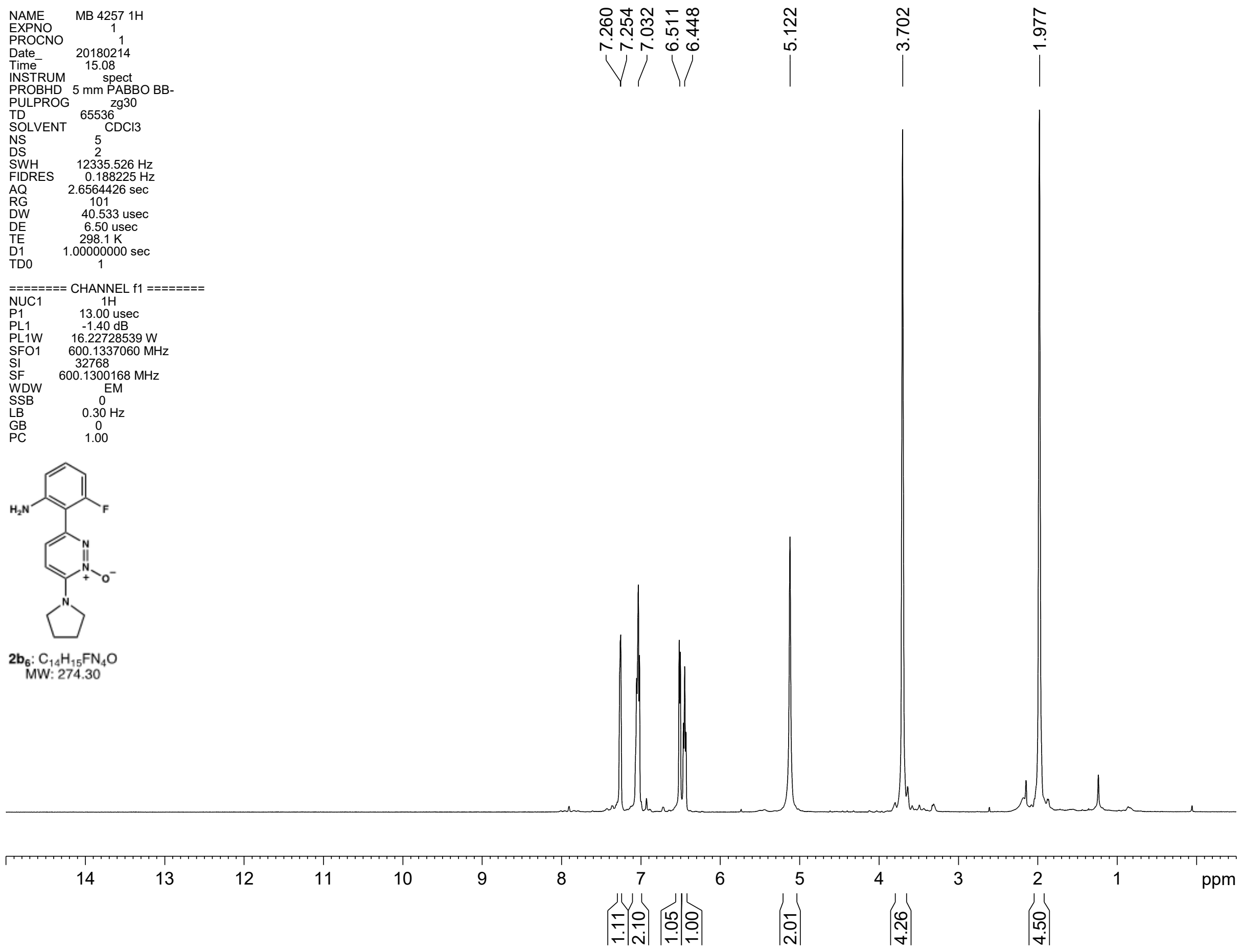




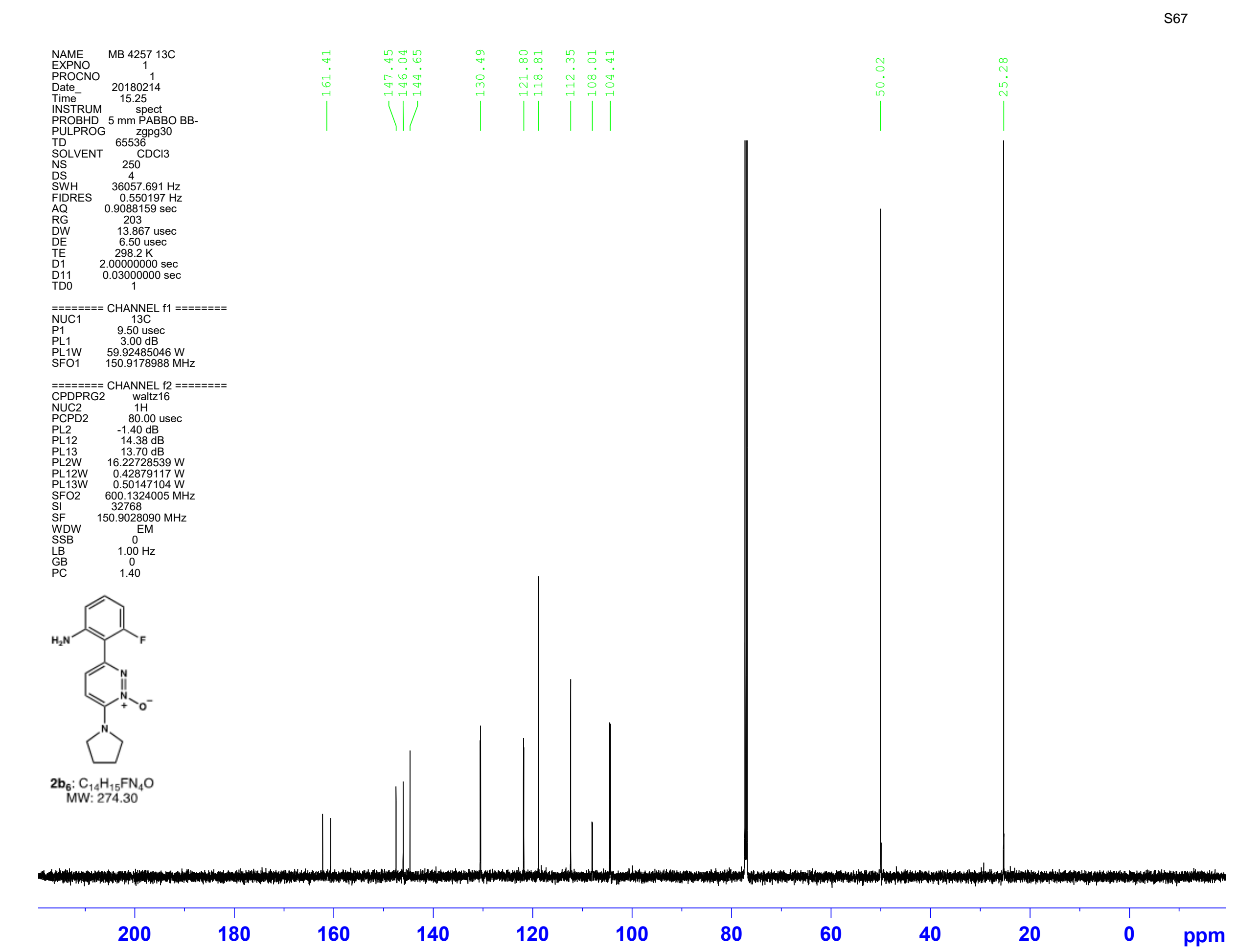




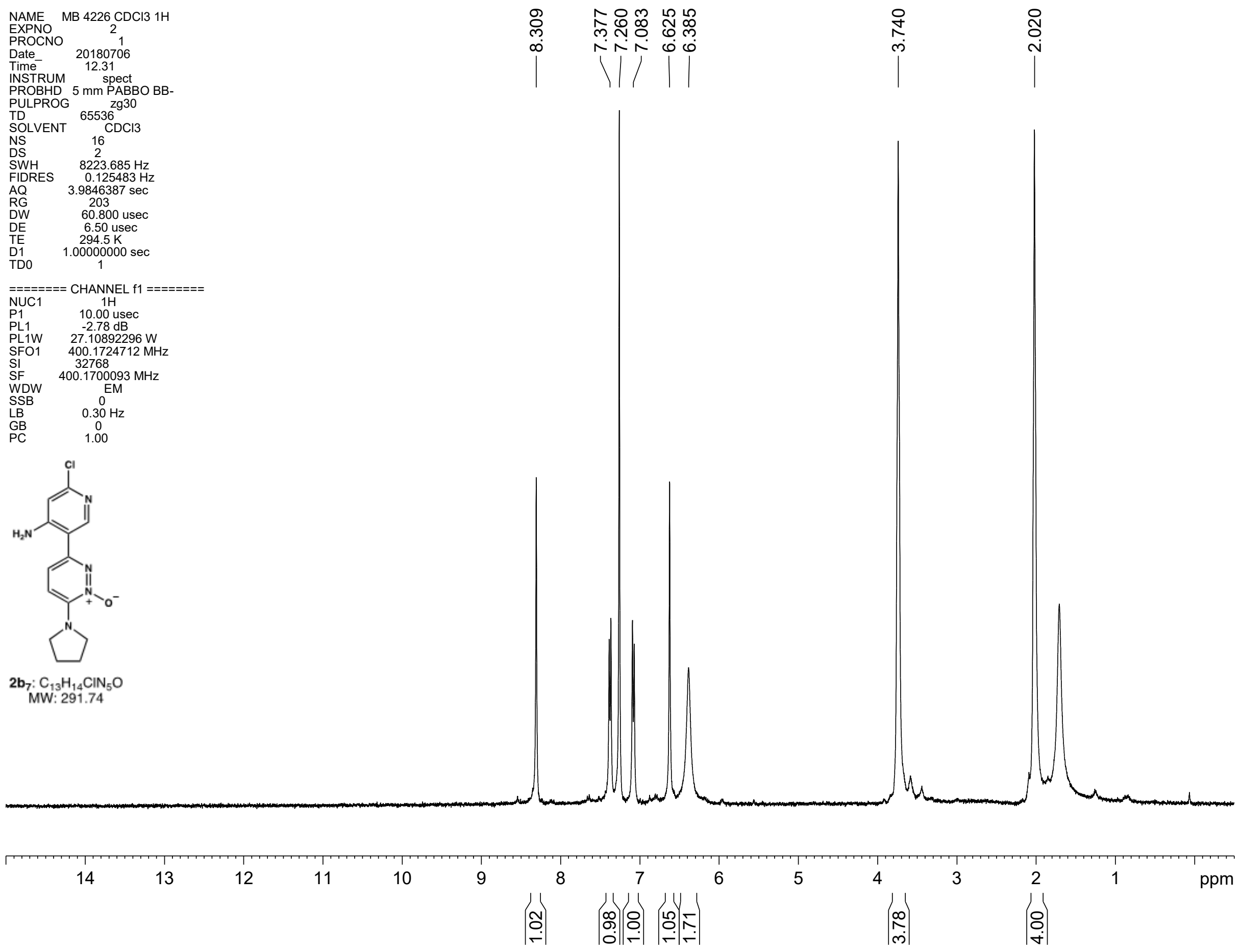





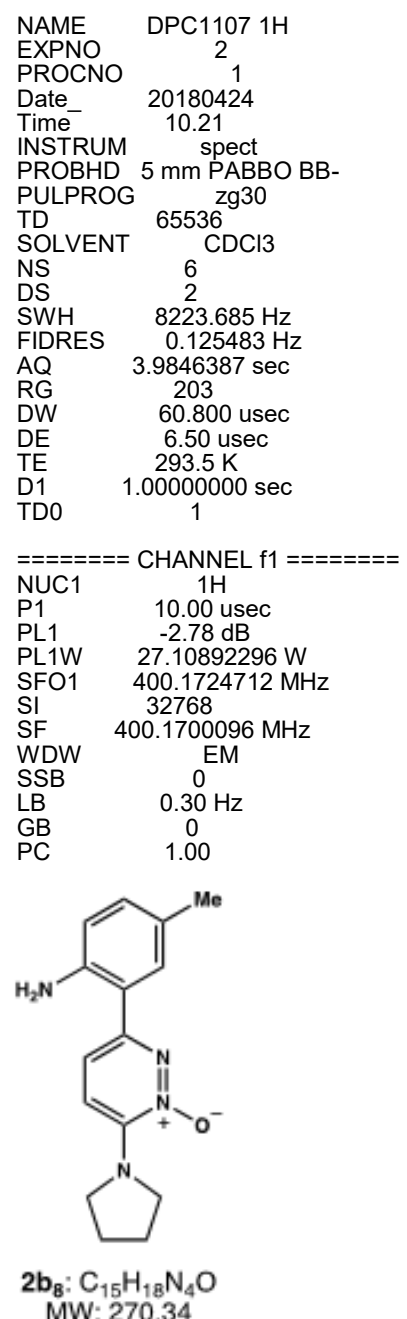

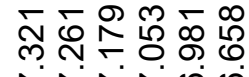

$\stackrel{\infty}{\infty}$

은

8 둥요

- 0

लि लं

술

mill

1

N
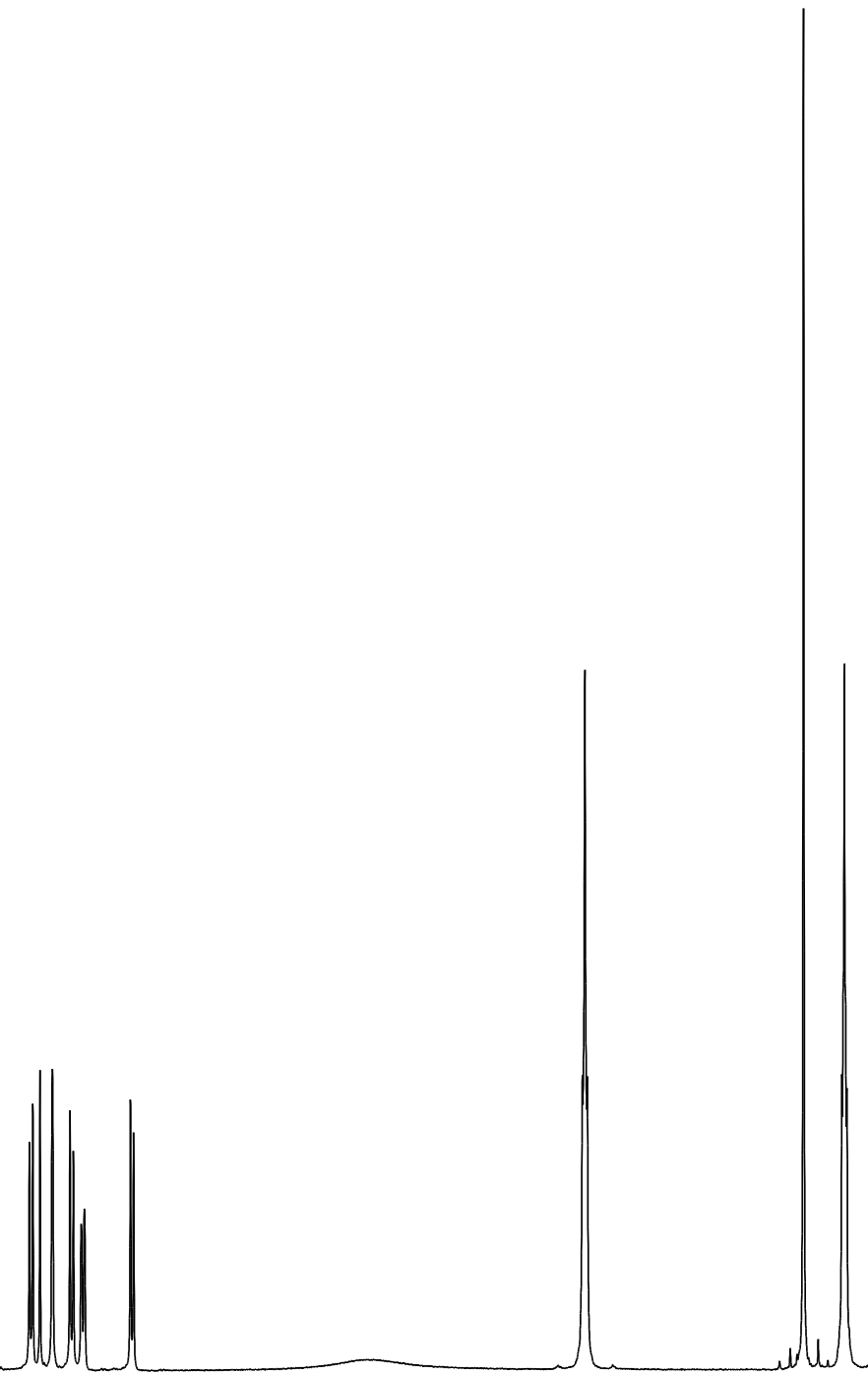

$14 \quad 13$

12

11

10

9

8

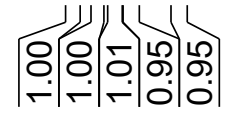

6

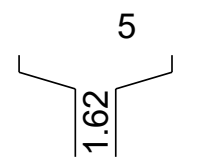

$\left|\begin{array}{l}\circ \\ 8 \\ \text { m. }\end{array}\right|$

3

2

$\left|\begin{array}{c}- \\ \infty \\ \dot{\infty}\end{array}\right| \begin{gathered}0 \\ 0 \\ m\end{gathered} \mid$ 

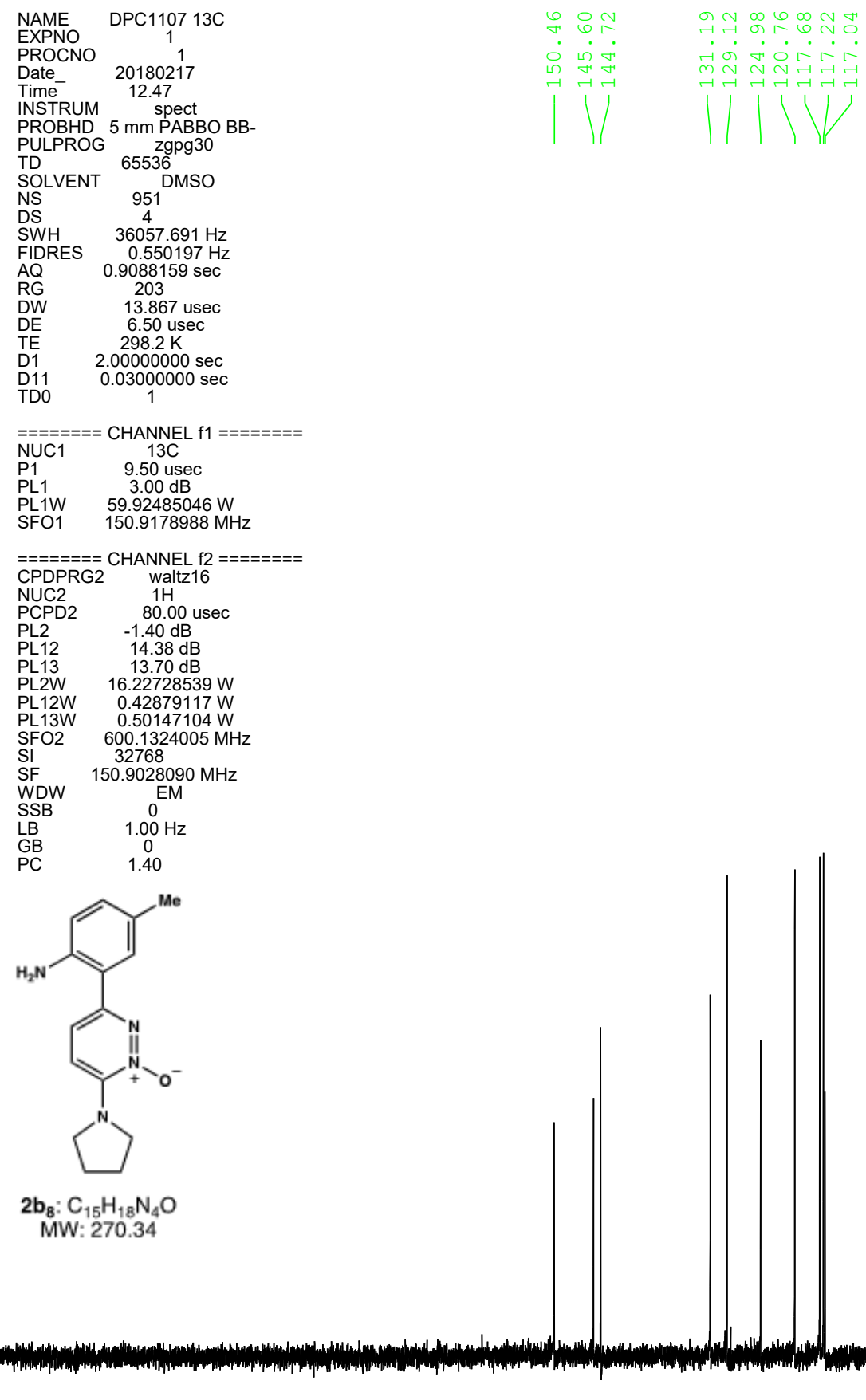


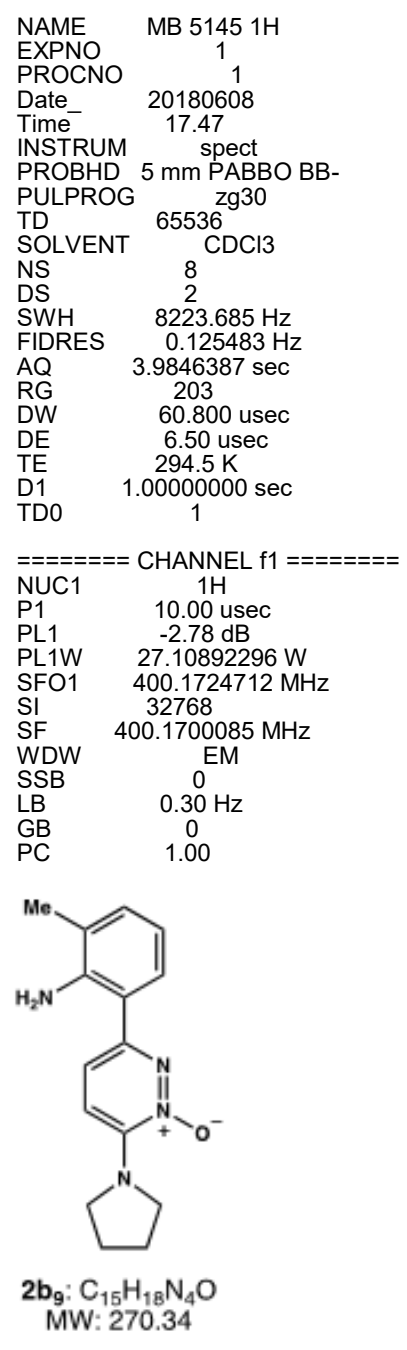

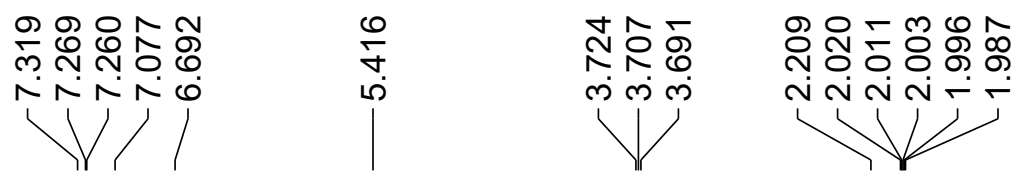

$14 \quad 13$

12

11

8

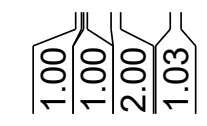

|

5

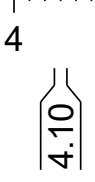

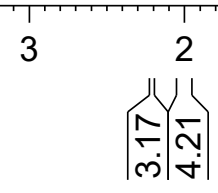




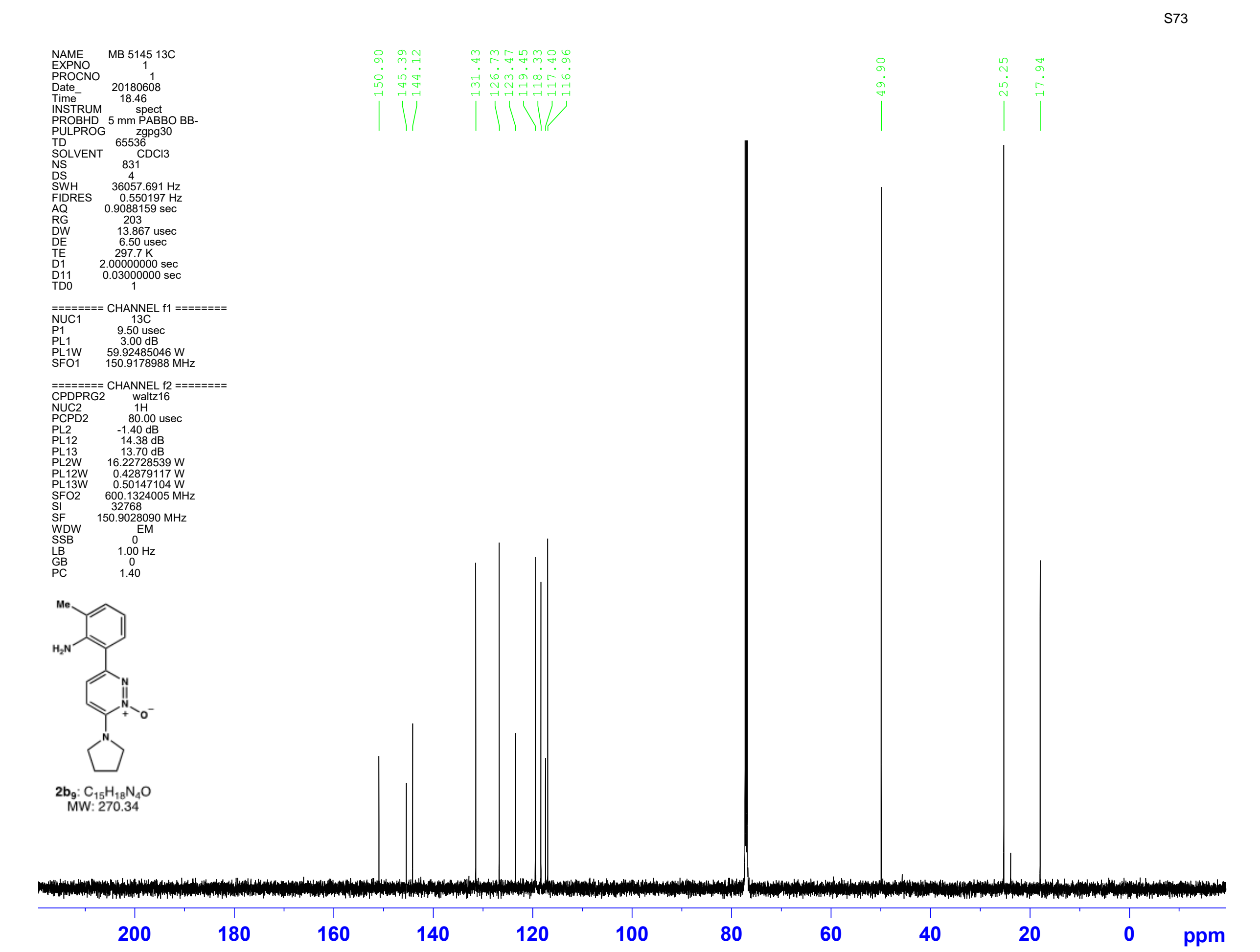




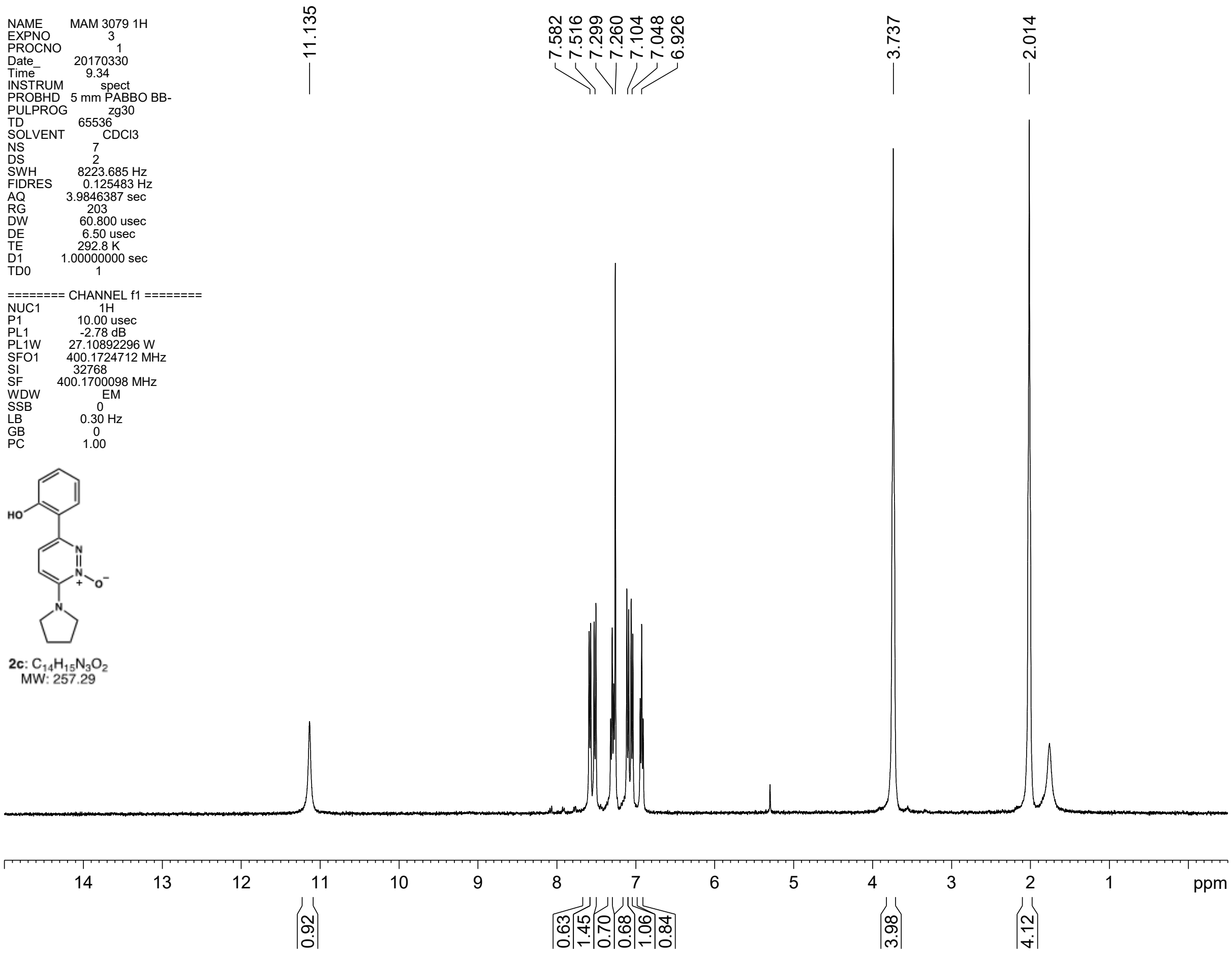




$$
\text { In }
$$




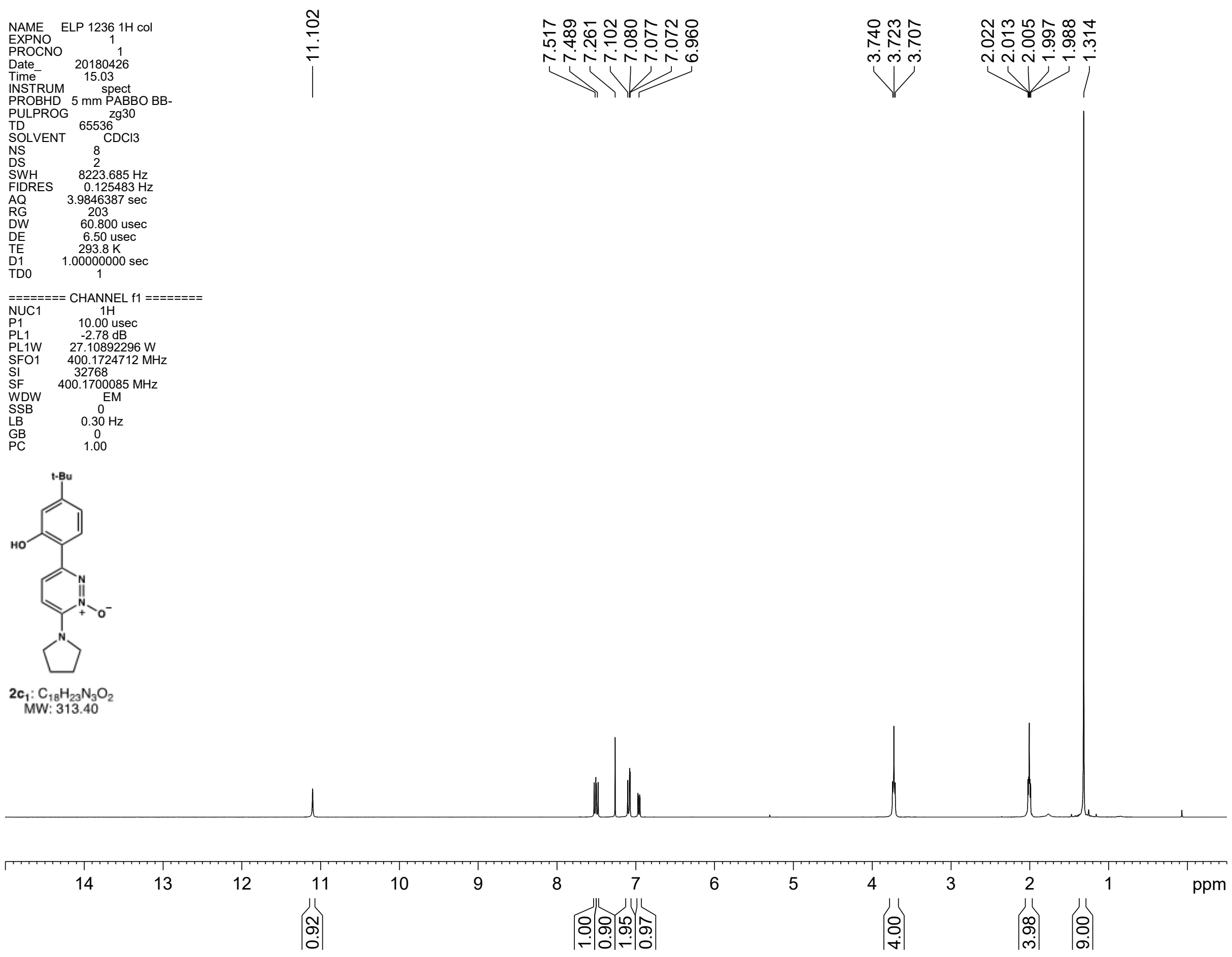




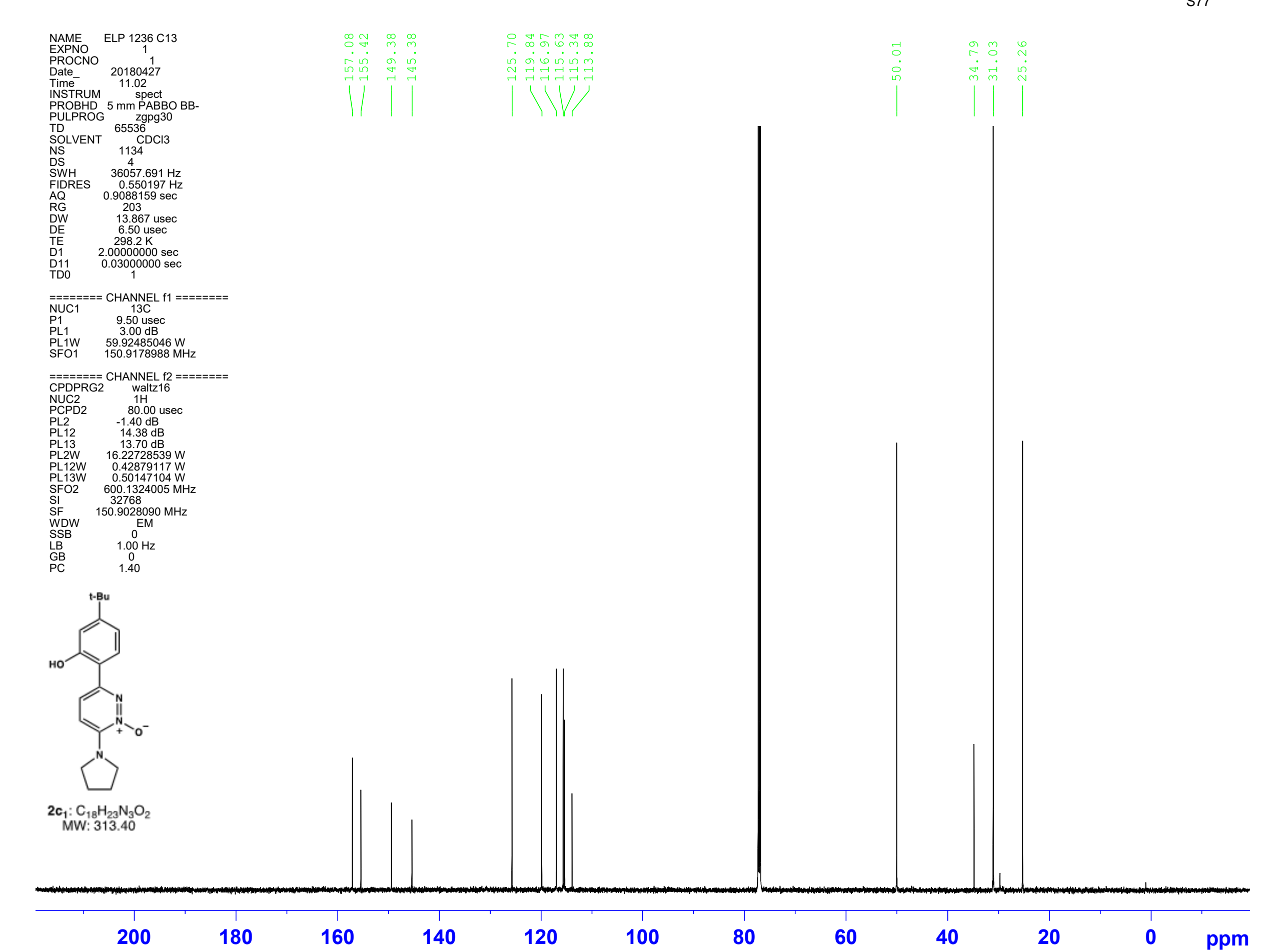




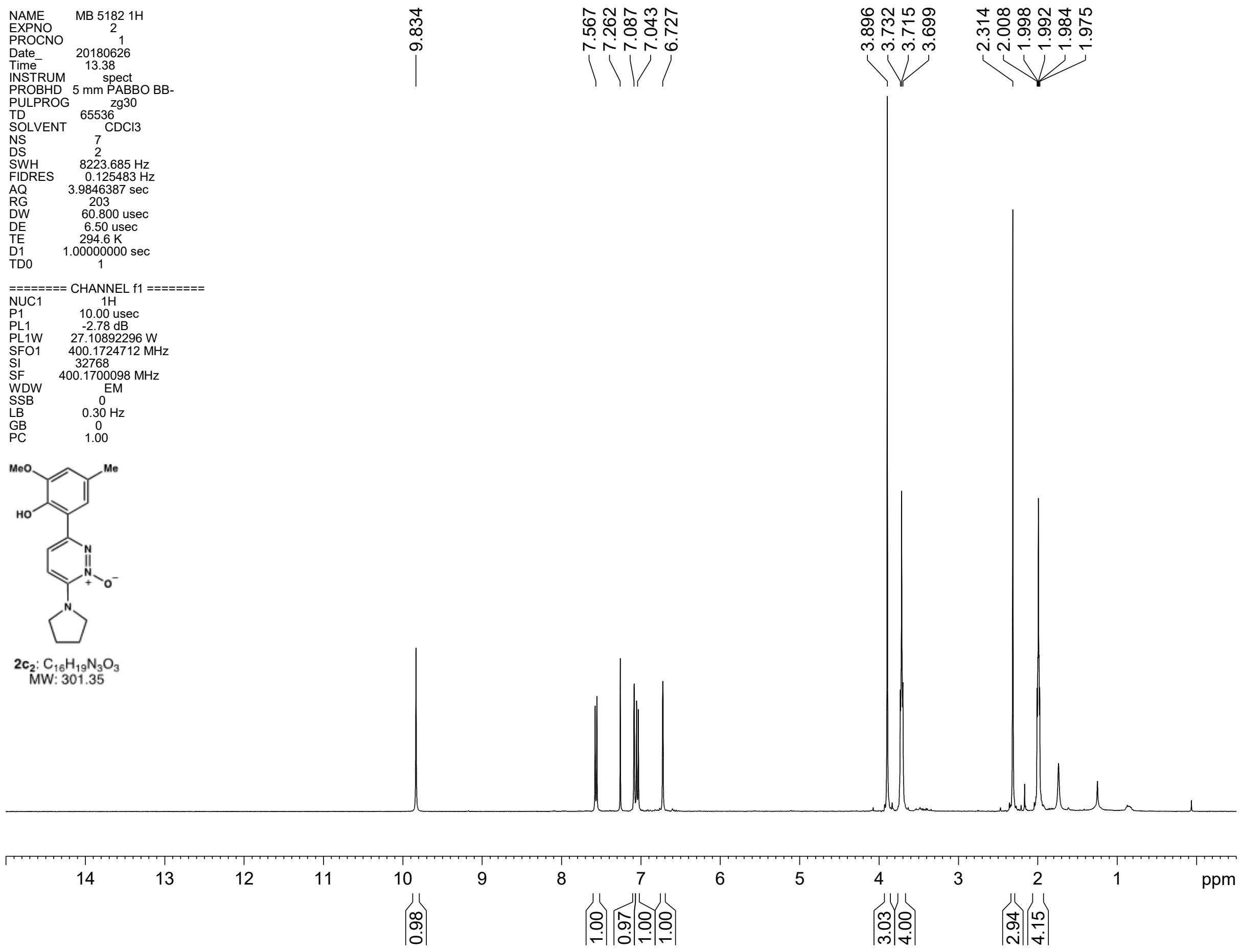



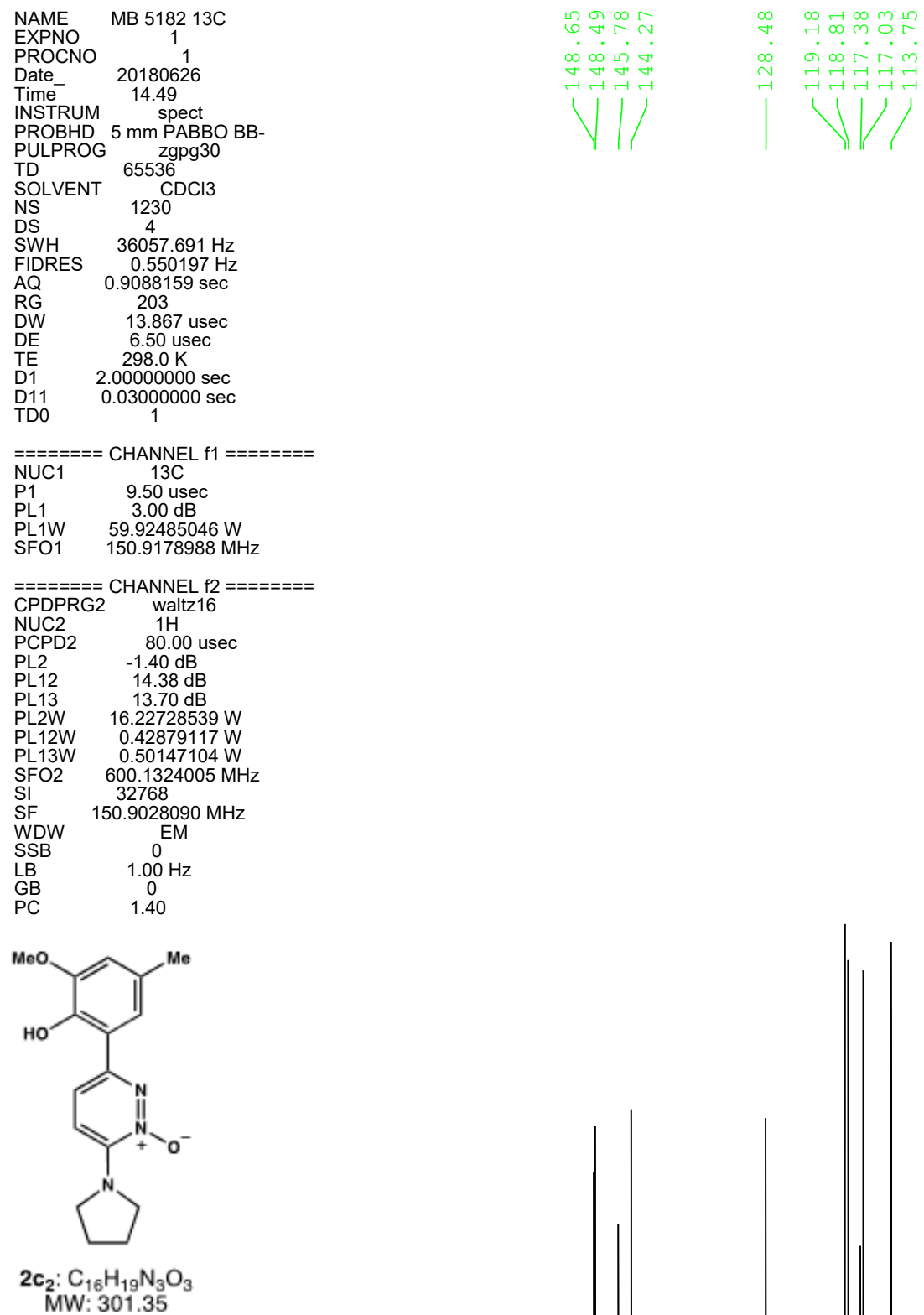

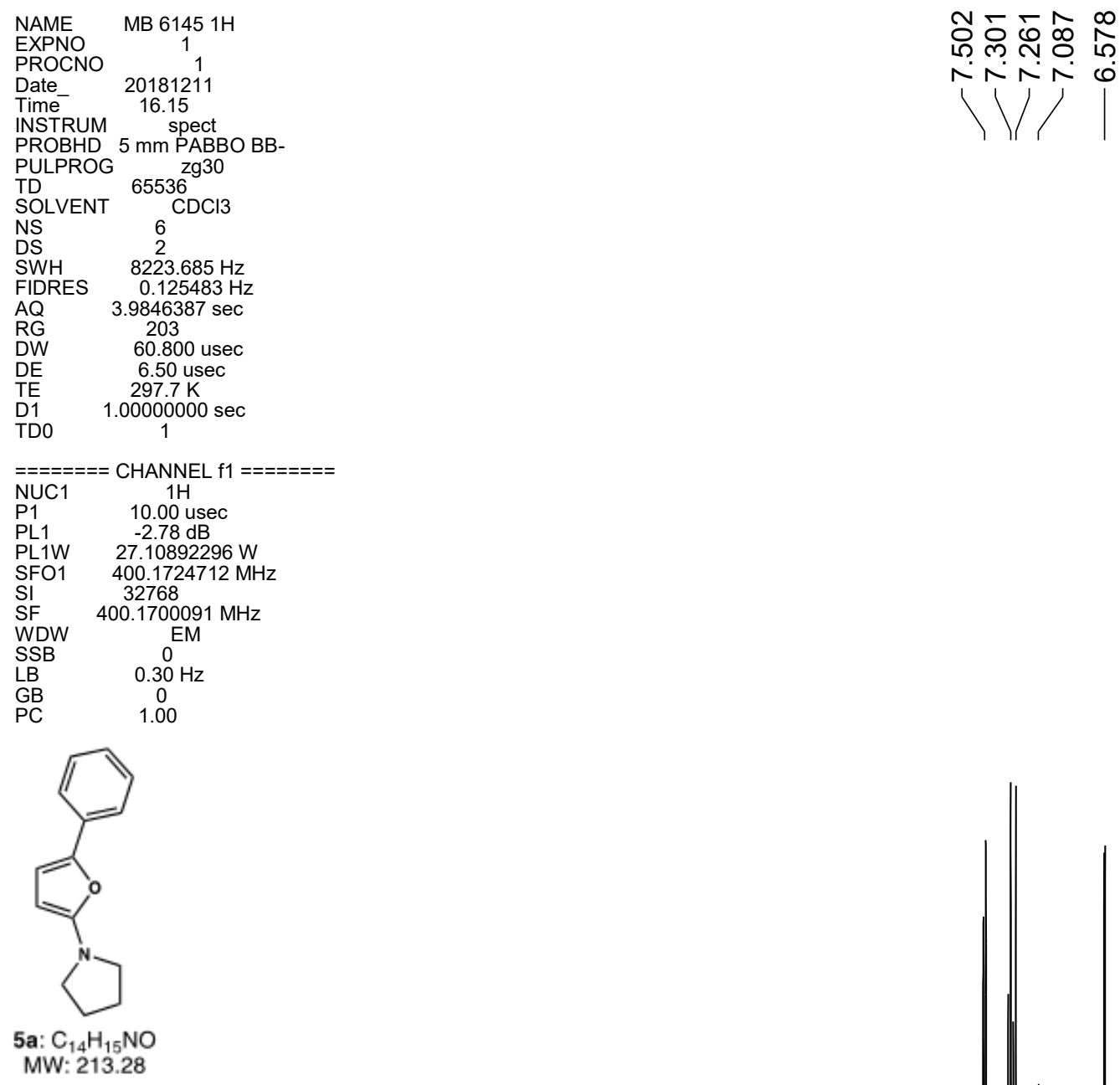

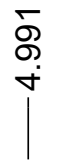

ભ

क्ष

PROBHD $5 \mathrm{~mm}$ PABBO BB-

PULPROG 2930

SOLVENT CDCl3

$=======$ CHANNEL $f 1$ =======

SFO1 $\quad 400.1724712 \mathrm{MHz}$

SF $\quad 400.1700091 \mathrm{MHz}$

WDW

GB $\quad 0.30 \mathrm{~Hz}$

MW: 213.28

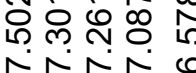

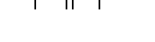

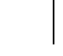

1

(n)

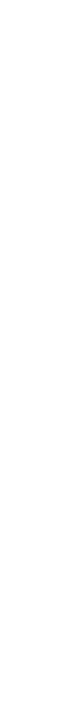




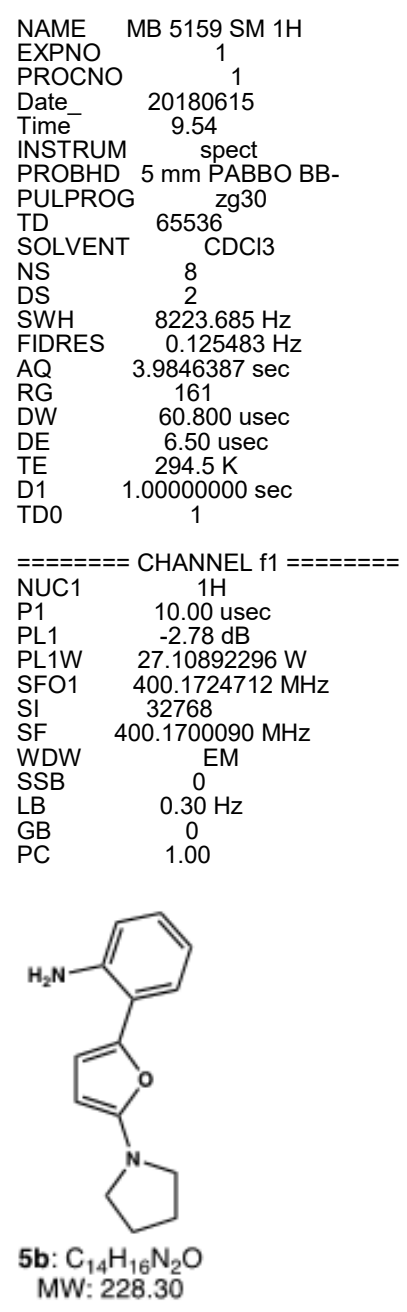

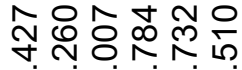

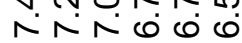

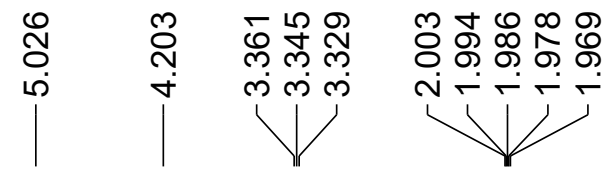

MW: 228.30

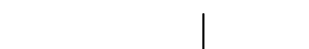

SWH

$=======$ CHANNEL $\mathrm{f} 1=======$

EM

$0.30 \mathrm{~Hz}$

1.00

13

12

11

10

10

9

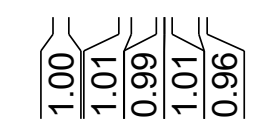

6

|人

4

$\left|\begin{array}{c}n \\ 0 \\ j\end{array}\right|$

2

$\left|\begin{array}{l}m \\ 0 \\ j\end{array}\right|$ 


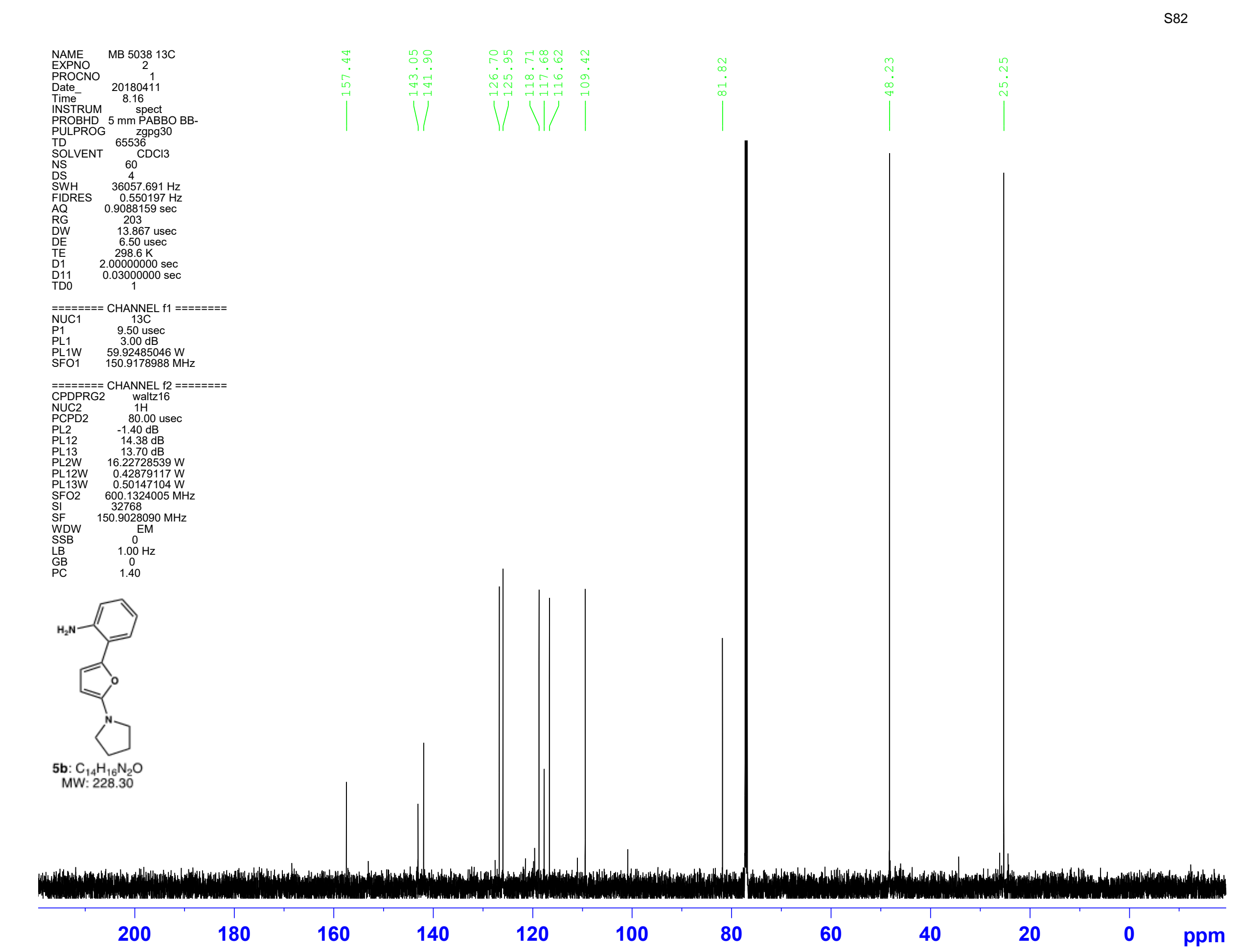




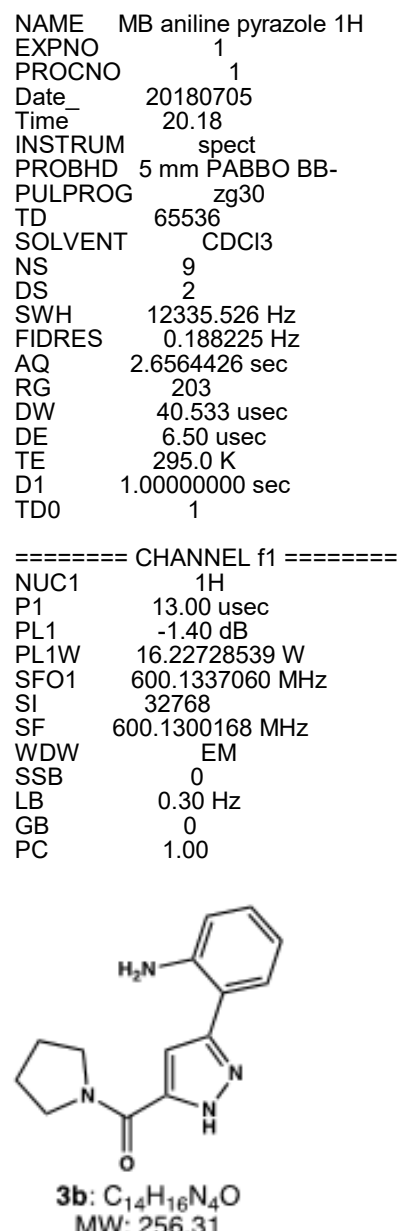

MW: 256.31
SWH

$=======$ CHANNEL $\mathrm{f} 1===== \pm==$

$-1.40 \mathrm{~dB}$

PL1W $16.22728539 \mathrm{~W}$

SI 32768

WDW

$\mathrm{LB}$

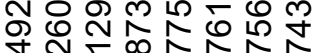

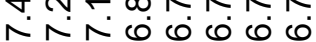
$<1<$

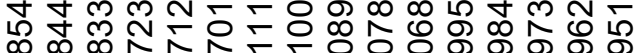
мं < l L l l l 

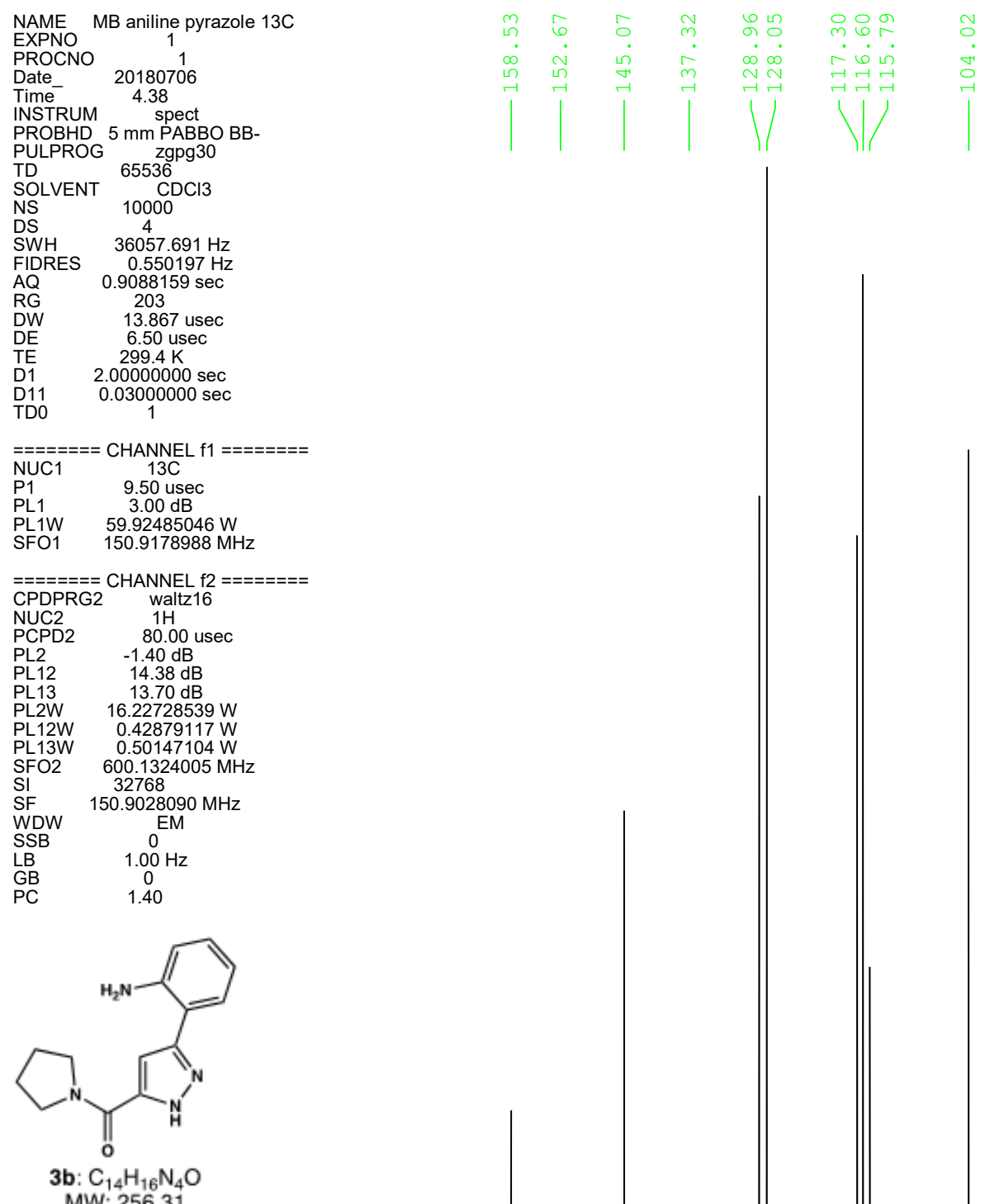

PULPOG $259 \mathrm{gg} 3$

CD

SWH $\quad 36057.691 \mathrm{~Hz}$

$\begin{array}{ll}\text { RG } & 203 \\ \text { DW } & 13.867 \text { usec }\end{array}$

$\begin{array}{ll}\mathrm{DE} & 6.50 \mathrm{us} \\ \mathrm{TE} & 299.4 \mathrm{~K}\end{array}$

D11 $\quad 0.00000000$

SFO1 $150.9178988 \mathrm{MHz}$

NUC2 $\quad 1 \mathrm{H}$

$\begin{array}{ll}\mathrm{SSB} & 0 \\ \mathrm{LB} & 1.00 \mathrm{~Hz} \\ \mathrm{~GB} & 0 \\ \mathrm{PC} & 1.40\end{array}$

20

年
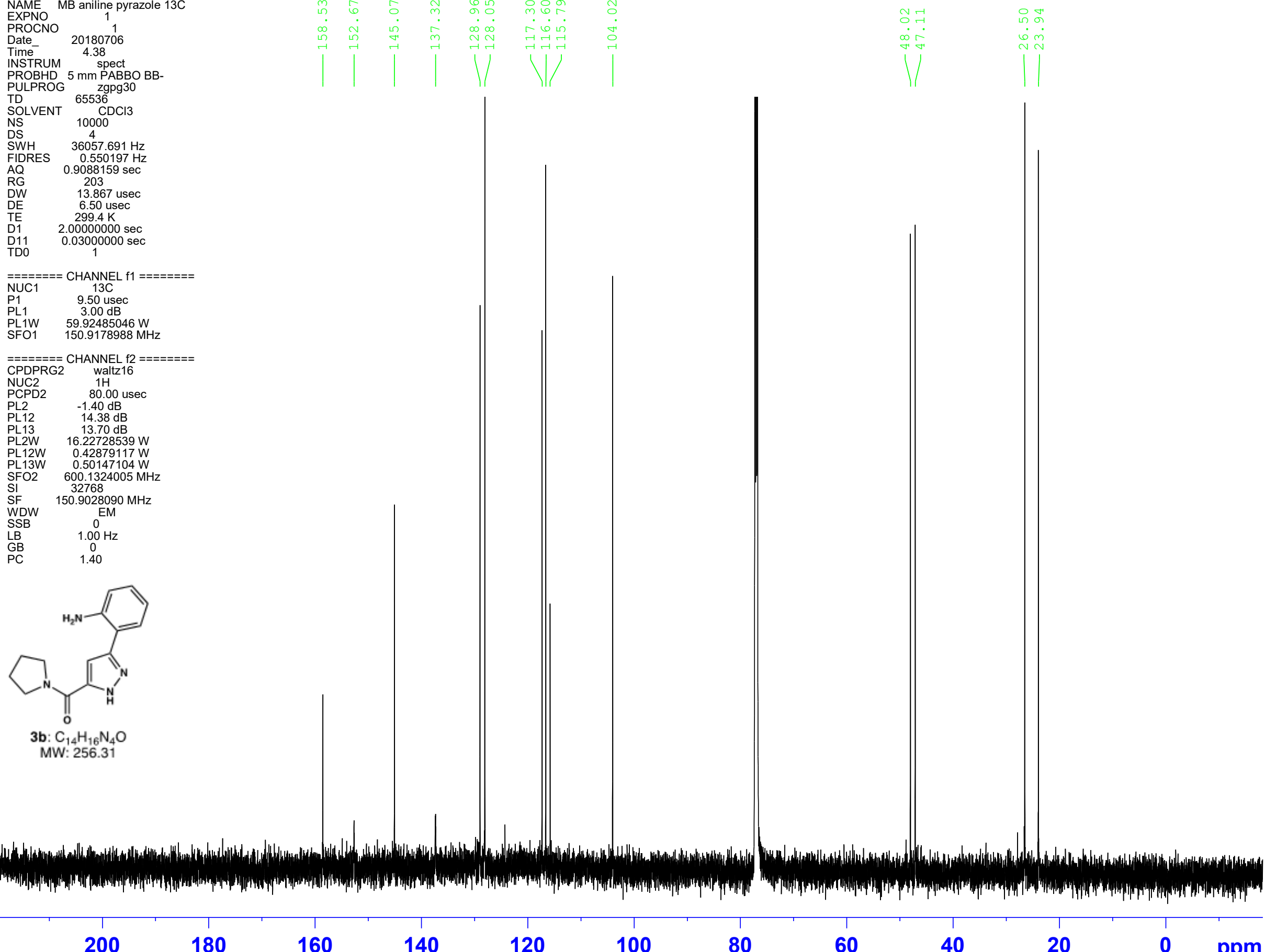

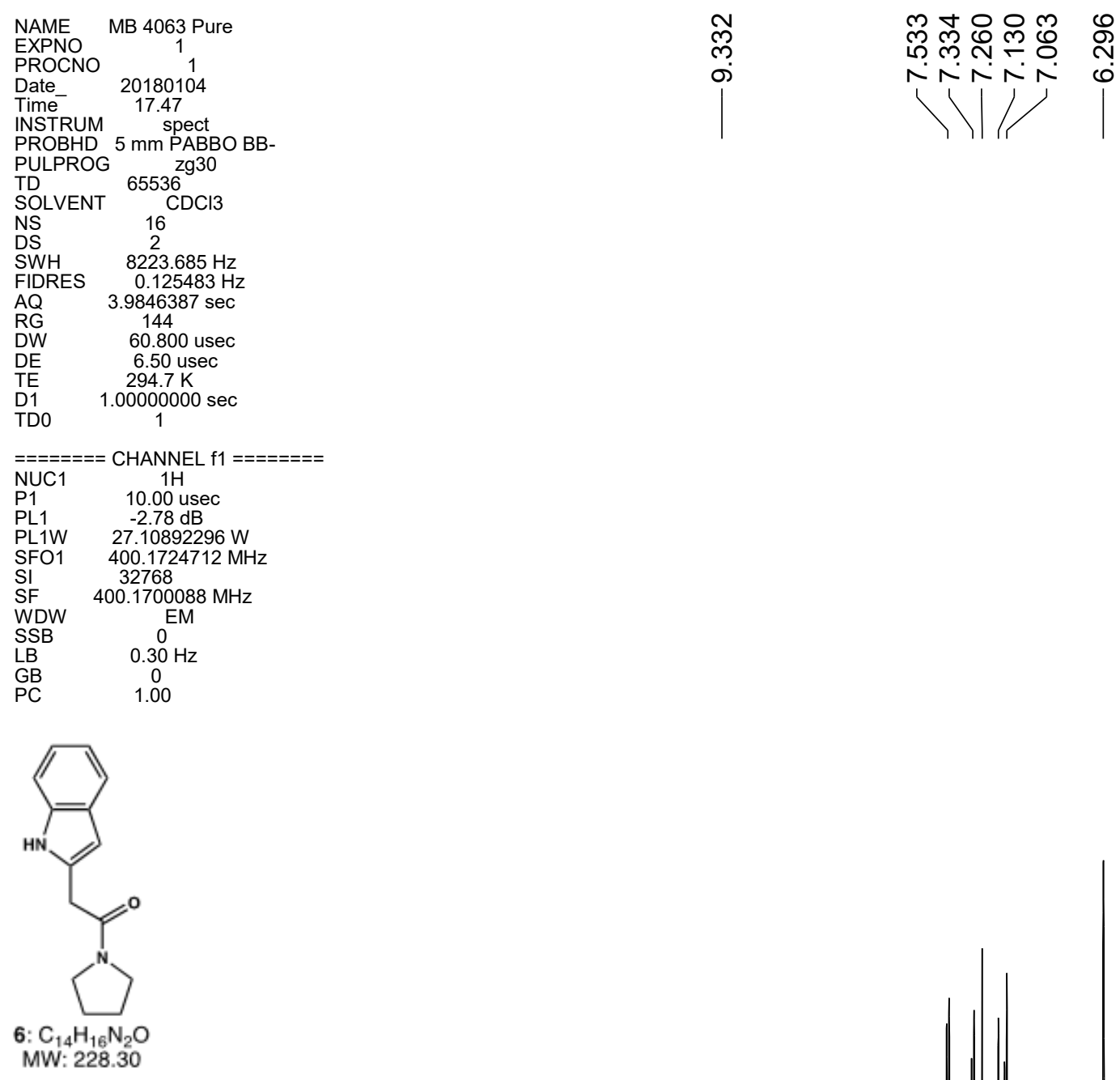

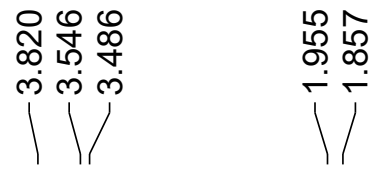

$M W: 228.30$

PROBHD $5 \mathrm{~mm}$ PABBO BB-

65536

SWH

NUC1 $1 \mathrm{H}$

SFO1 $\quad 400.1724712 \mathrm{MHz}$

SF $\quad 400.1700088 \mathrm{MHz}$

SSB EM

$\begin{array}{ll}\mathrm{GB} & 0.30 \mathrm{H} \\ \mathrm{PC} & 0 \\ \mathrm{PC} & 1.00\end{array}$

$\Lambda$

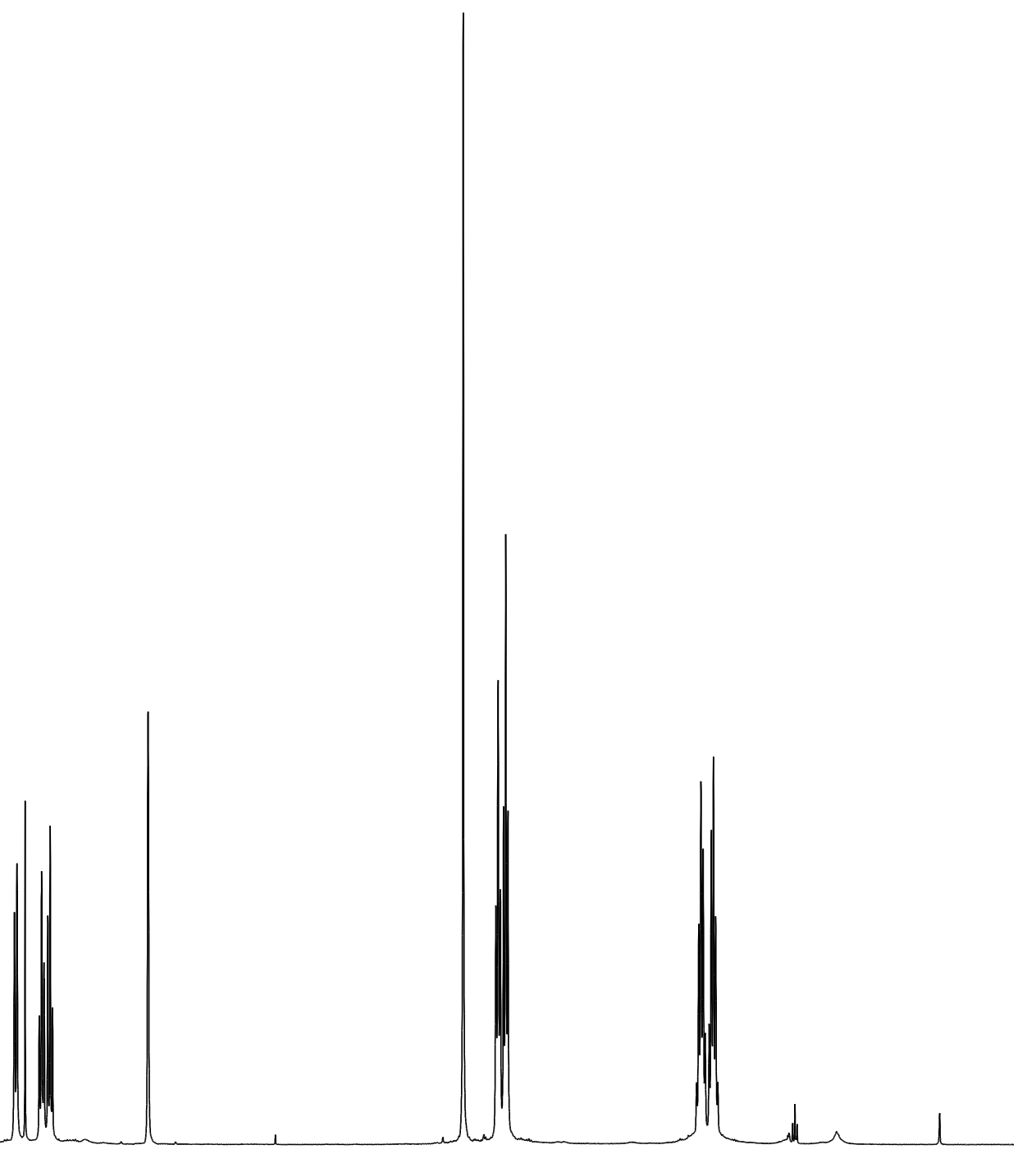

$14 \quad 13$

12

11

10

$\left.\right|^{9}$

8

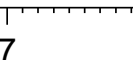

6

5

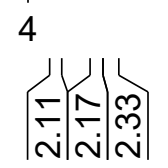

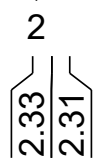




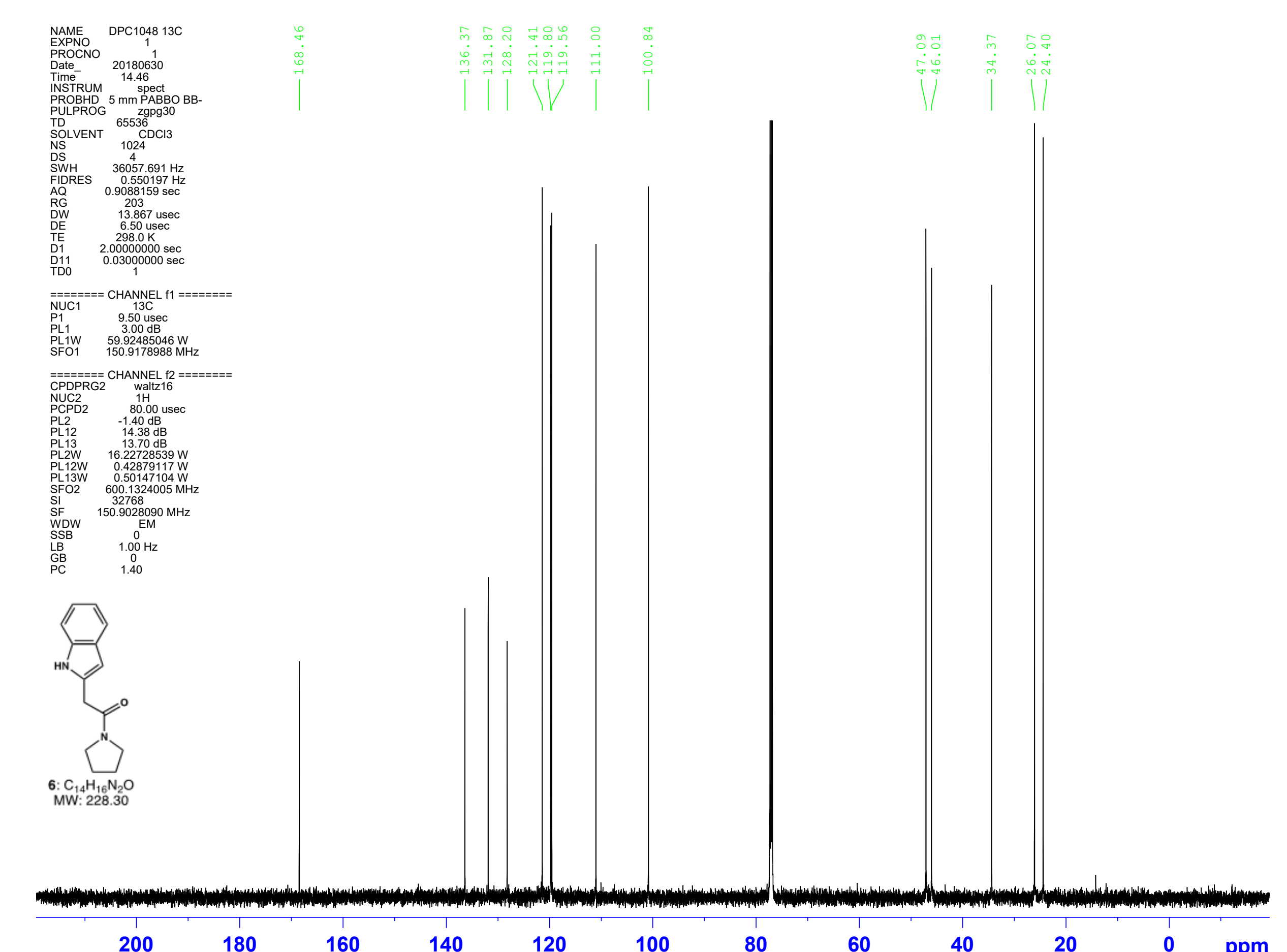



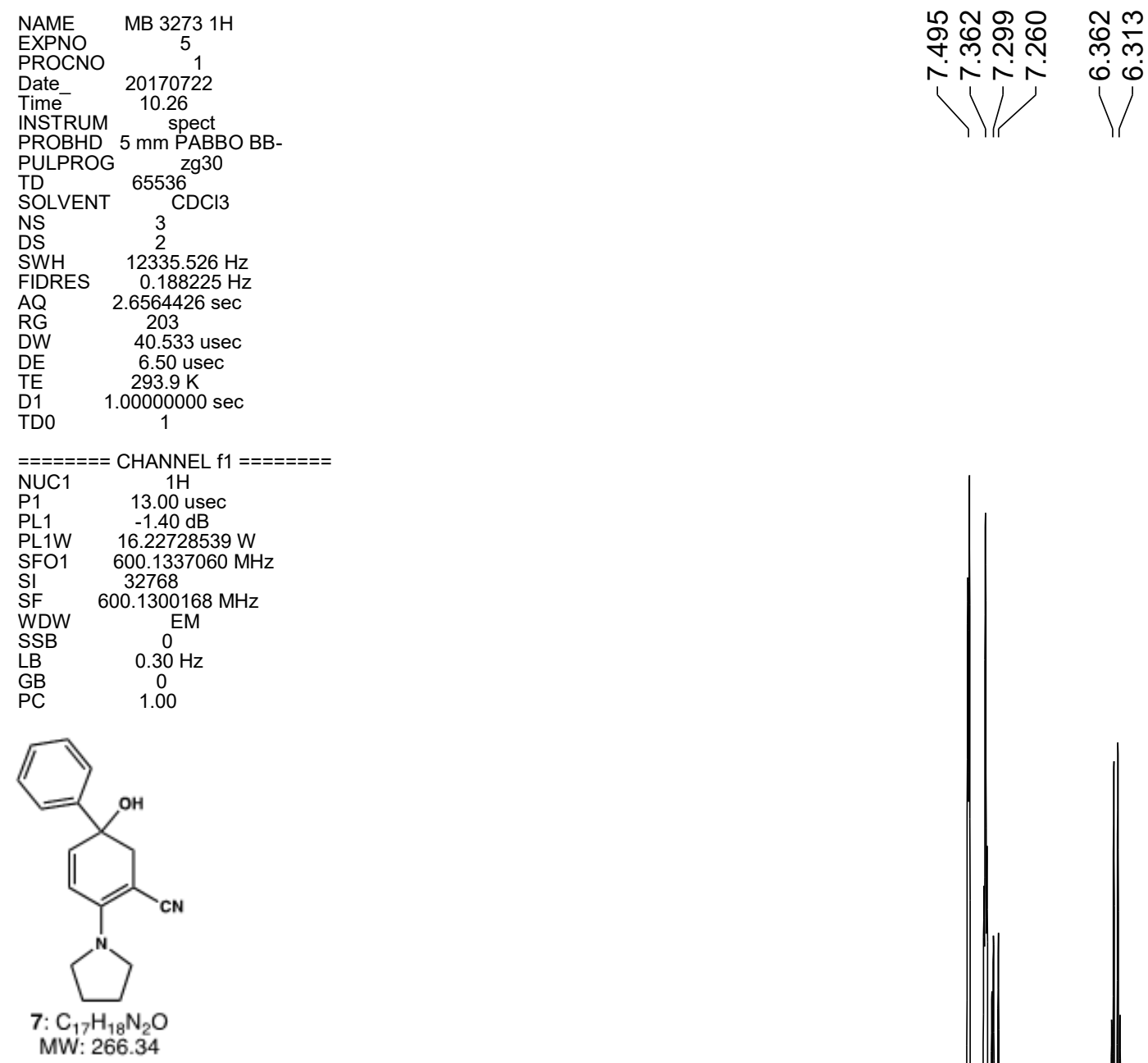

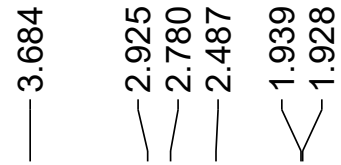

PROBHD $5 \mathrm{~mm}$ PABBO BB-

Zg30

SOLVENT CDCl3

DS

SWH $12335.526 \mathrm{~Hz}$

$\quad \mathrm{AQ} \quad 2.6564426 \mathrm{sec}$

DW 40.533 usec

1.000000

$=======$ CHANNEL $\mathrm{f} 1=======$

$-1.40 \mathrm{~dB}$

$\begin{array}{ll}\text { PL1W } & 16.22728539 \mathrm{~W} \\ \text { SFO1 } & 600.1337060 \mathrm{MHz}\end{array}$

$\begin{array}{ll}\text { Sl } & 32768 \\ \text { SF } & 600.1300168 \mathrm{MHz}\end{array}$

WDW

$\stackrel{L B}{G B} \quad 0.30 \mathrm{~Hz}$

MW: 266.34

jo




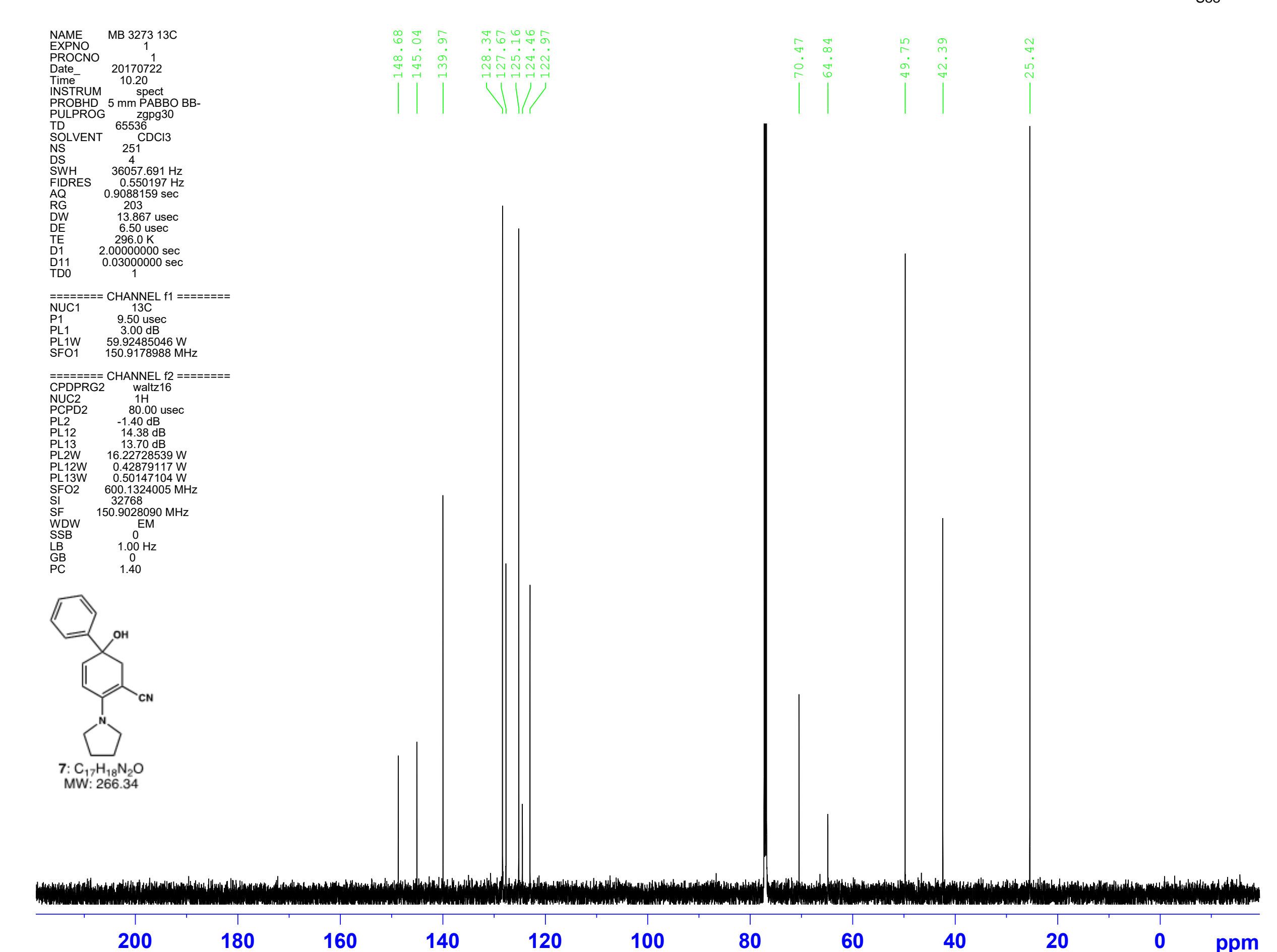




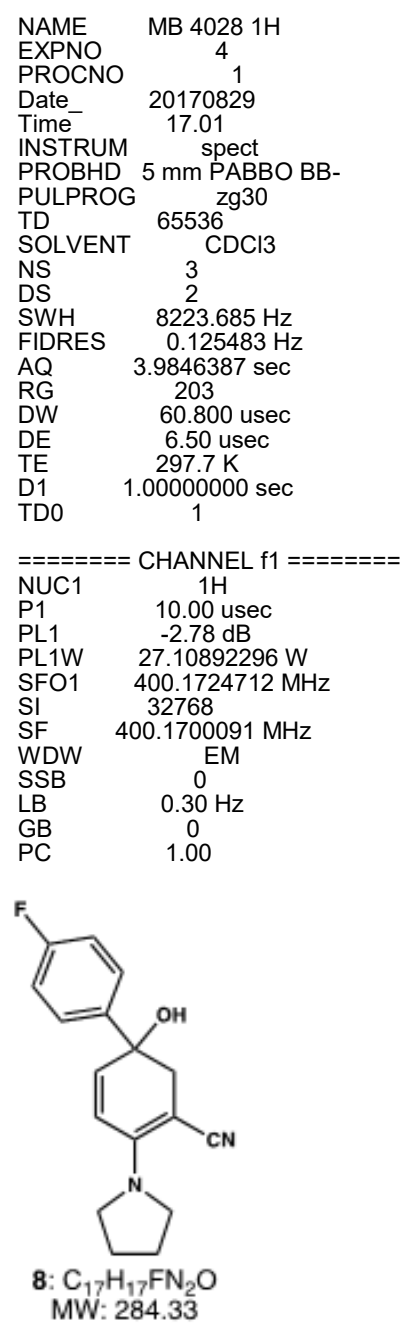

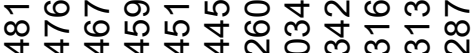

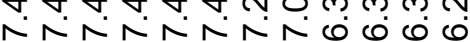

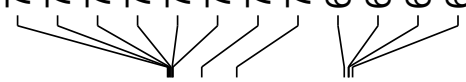

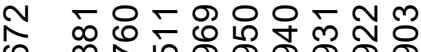

(i)

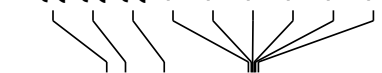




$$
\text { inll }
$$




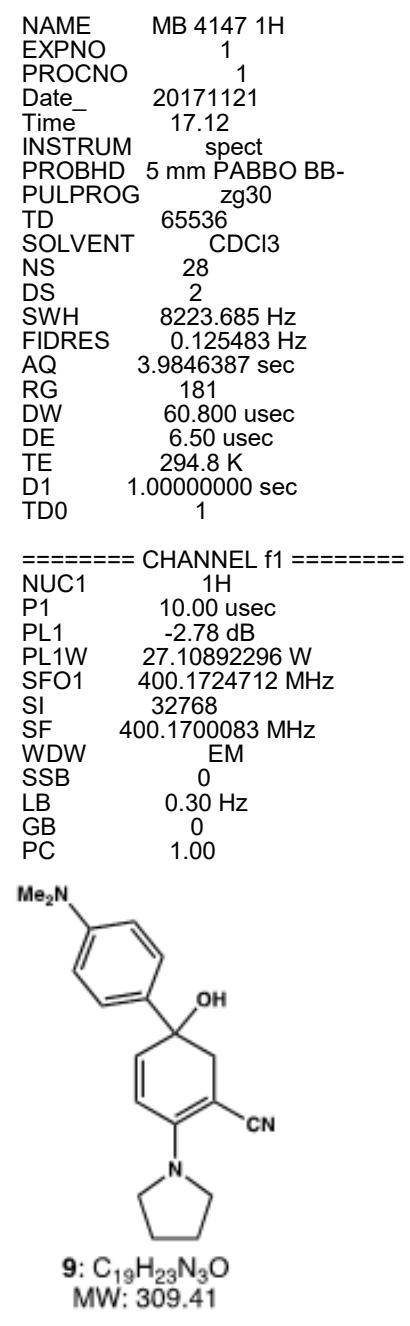

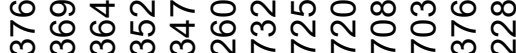
N N N

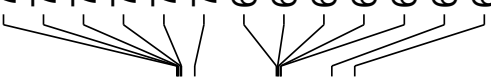

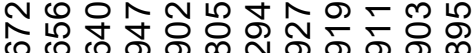
पि

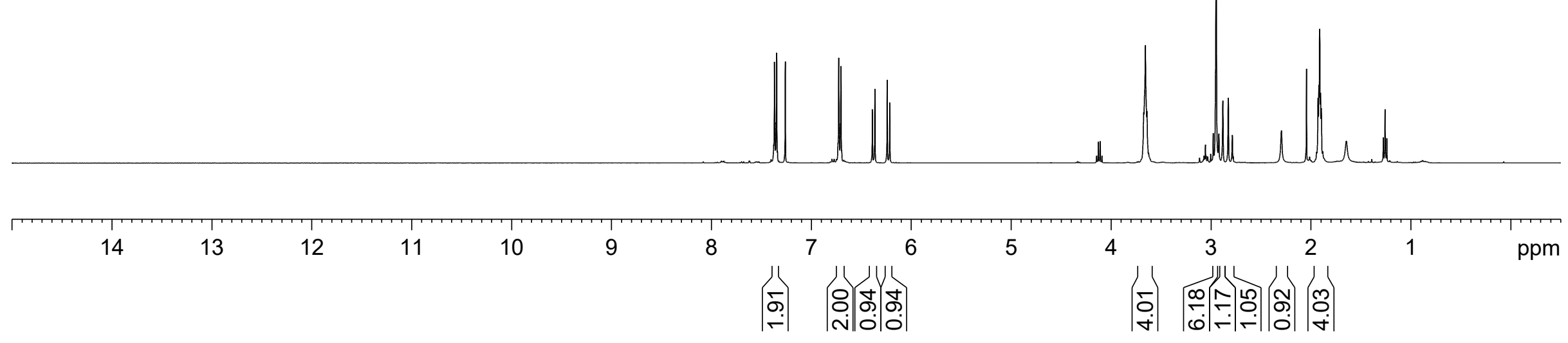




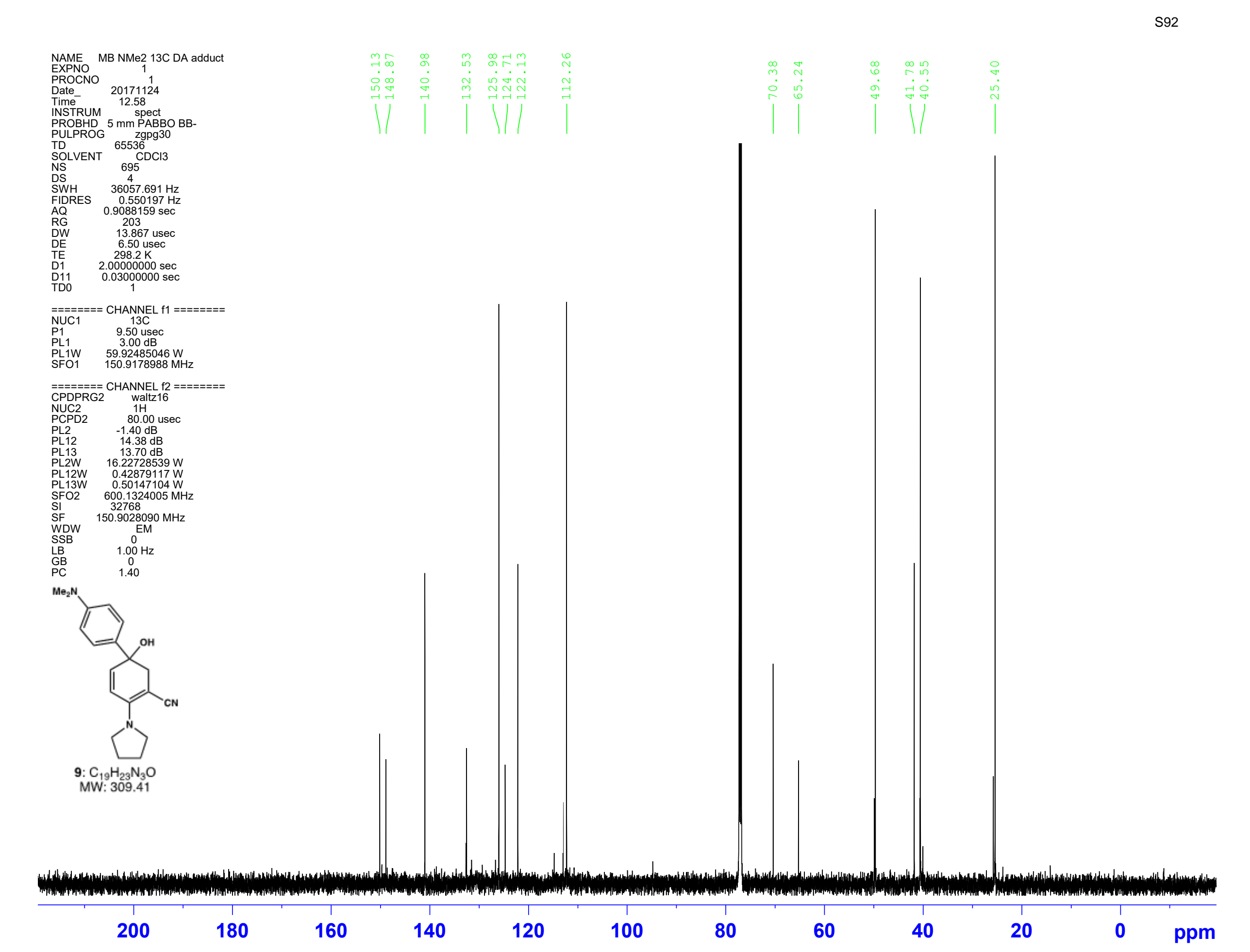




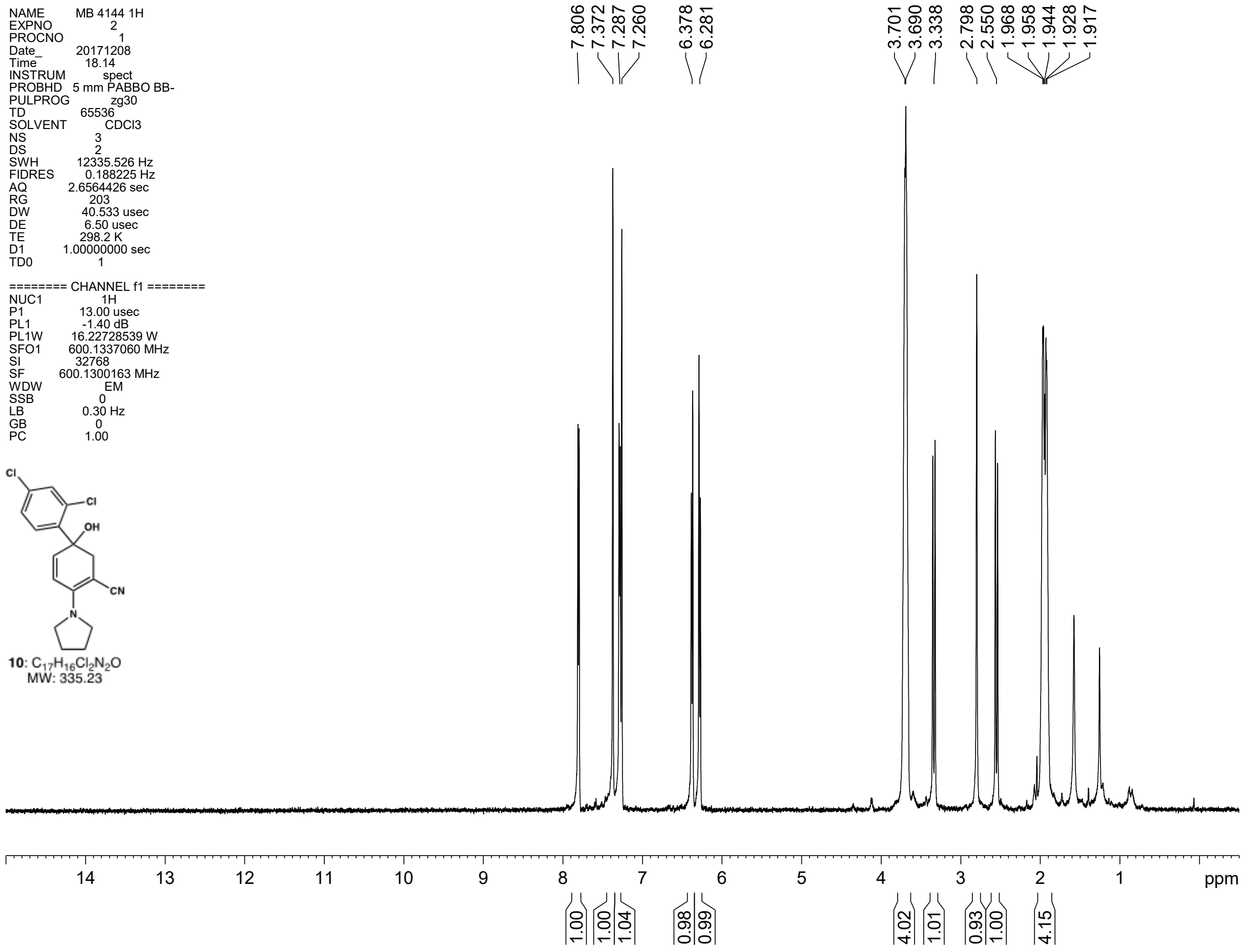




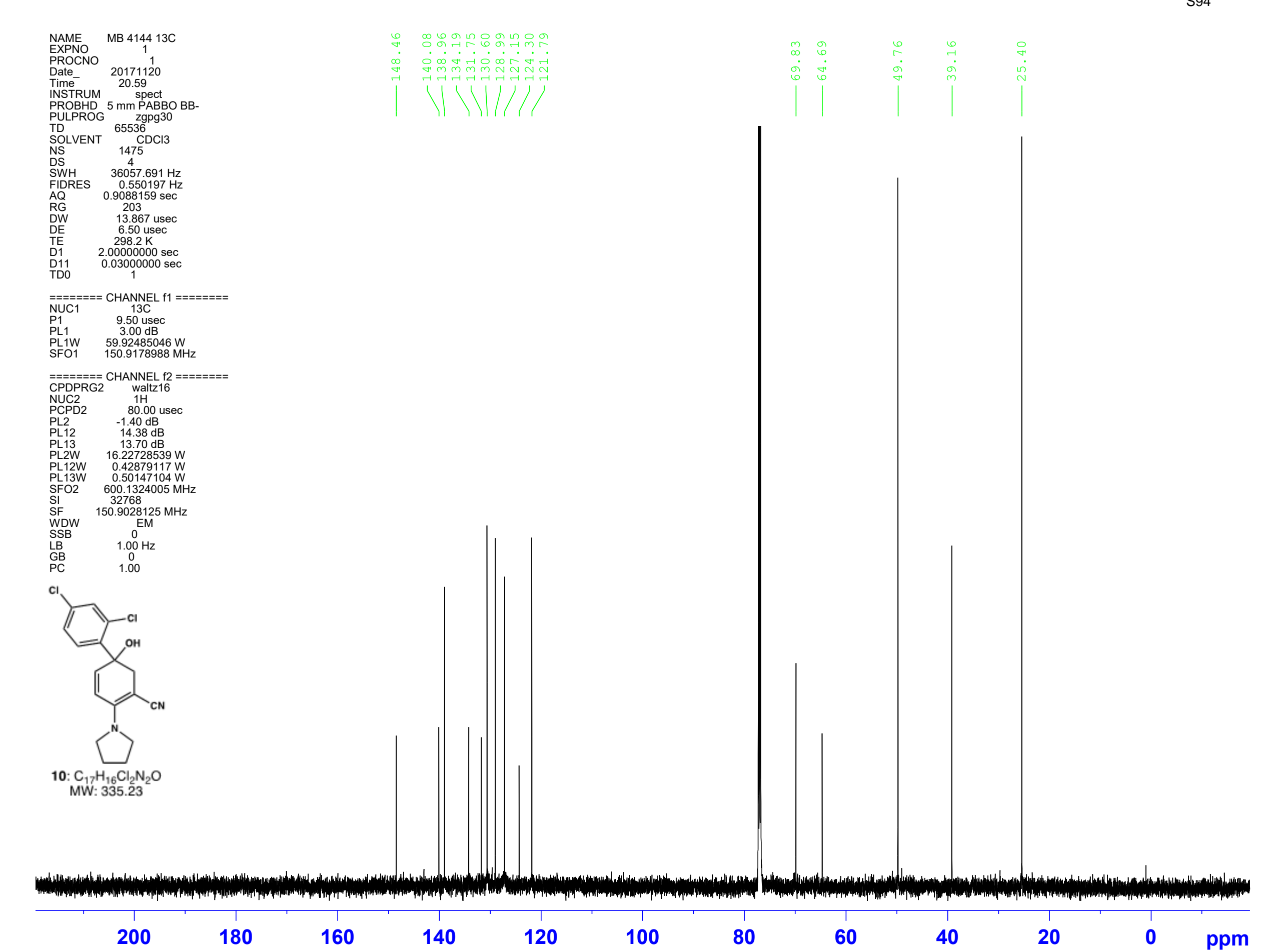




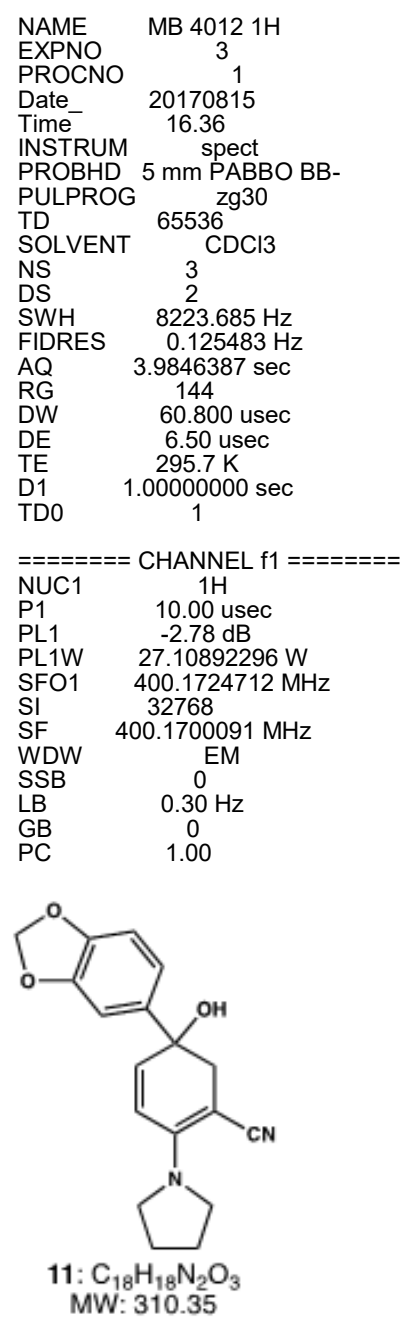

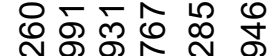
तें0ं

P

CDCI3

SWH $\quad 8223.685 \mathrm{~Hz}$

FWRES $0.125483 \mathrm{~Hz}$

RG $\quad 144$

DW $\quad 60.800$ usec

$295.7 \mathrm{~K}$

$=======$ CHANNEL $\mathrm{f} 1$ =======

$\mathrm{PC}$

$\mathrm{MW}: 310.35$

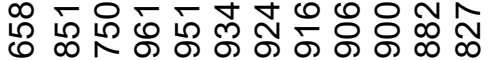

ن

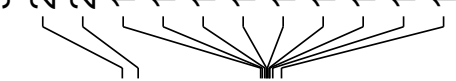

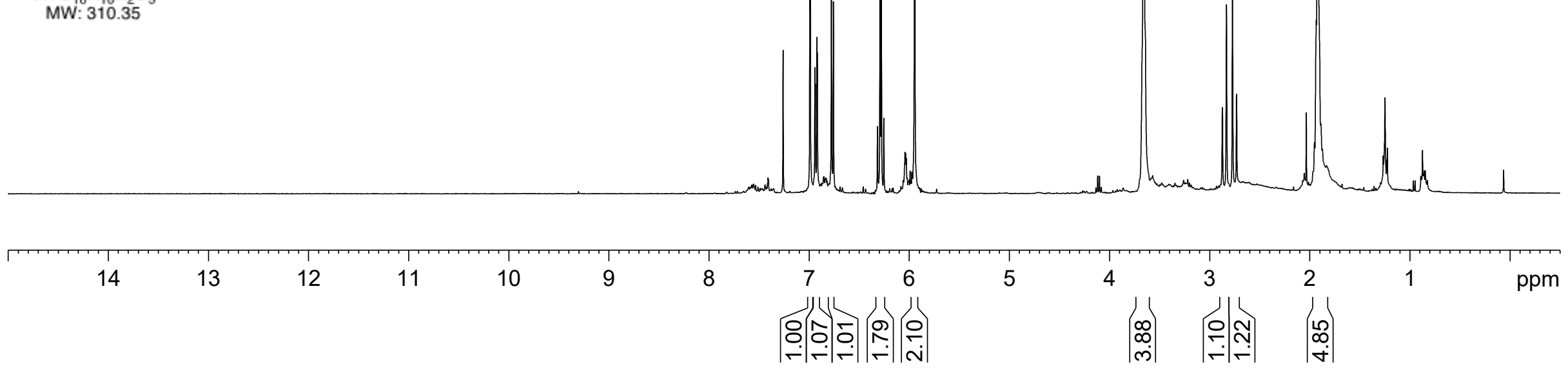




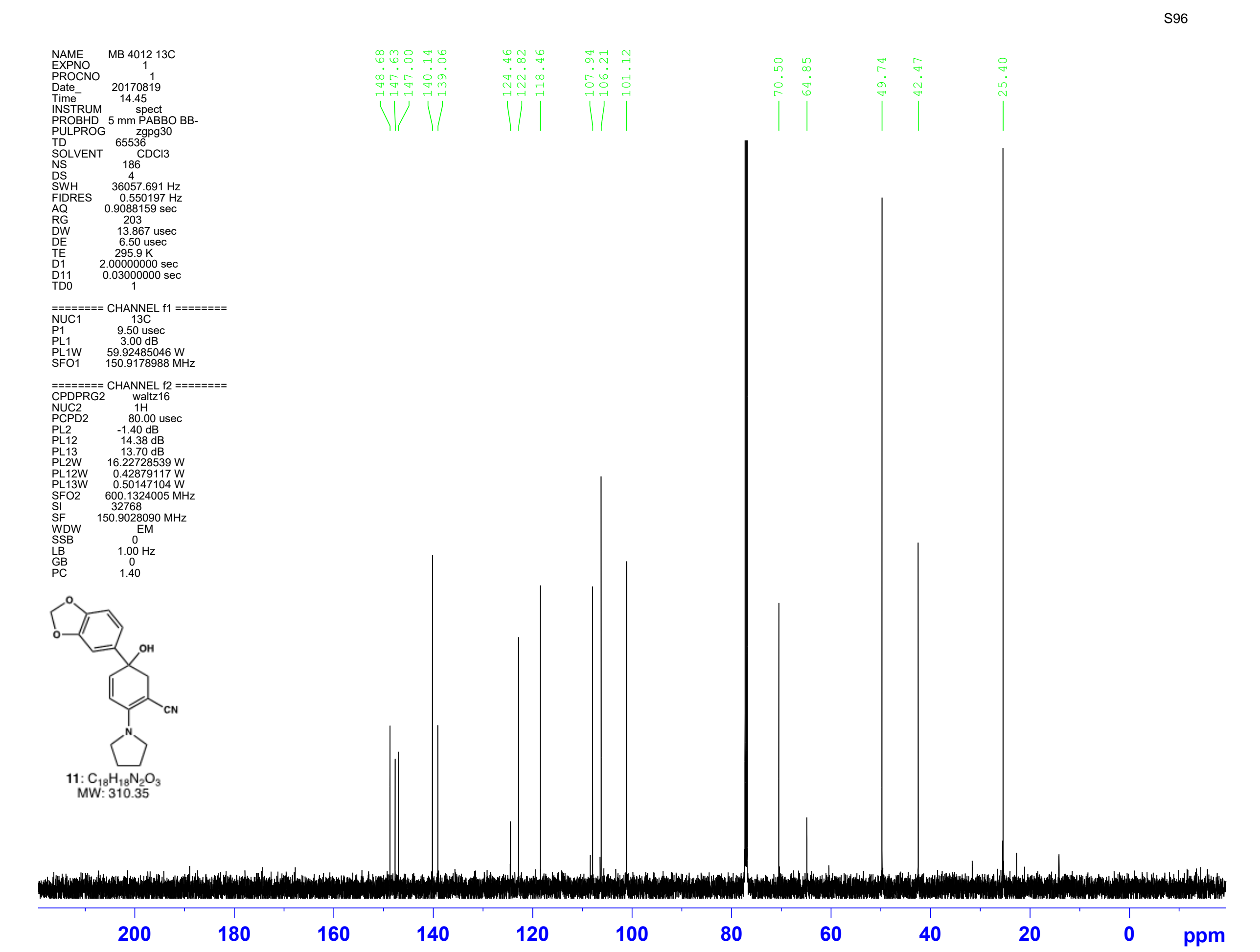




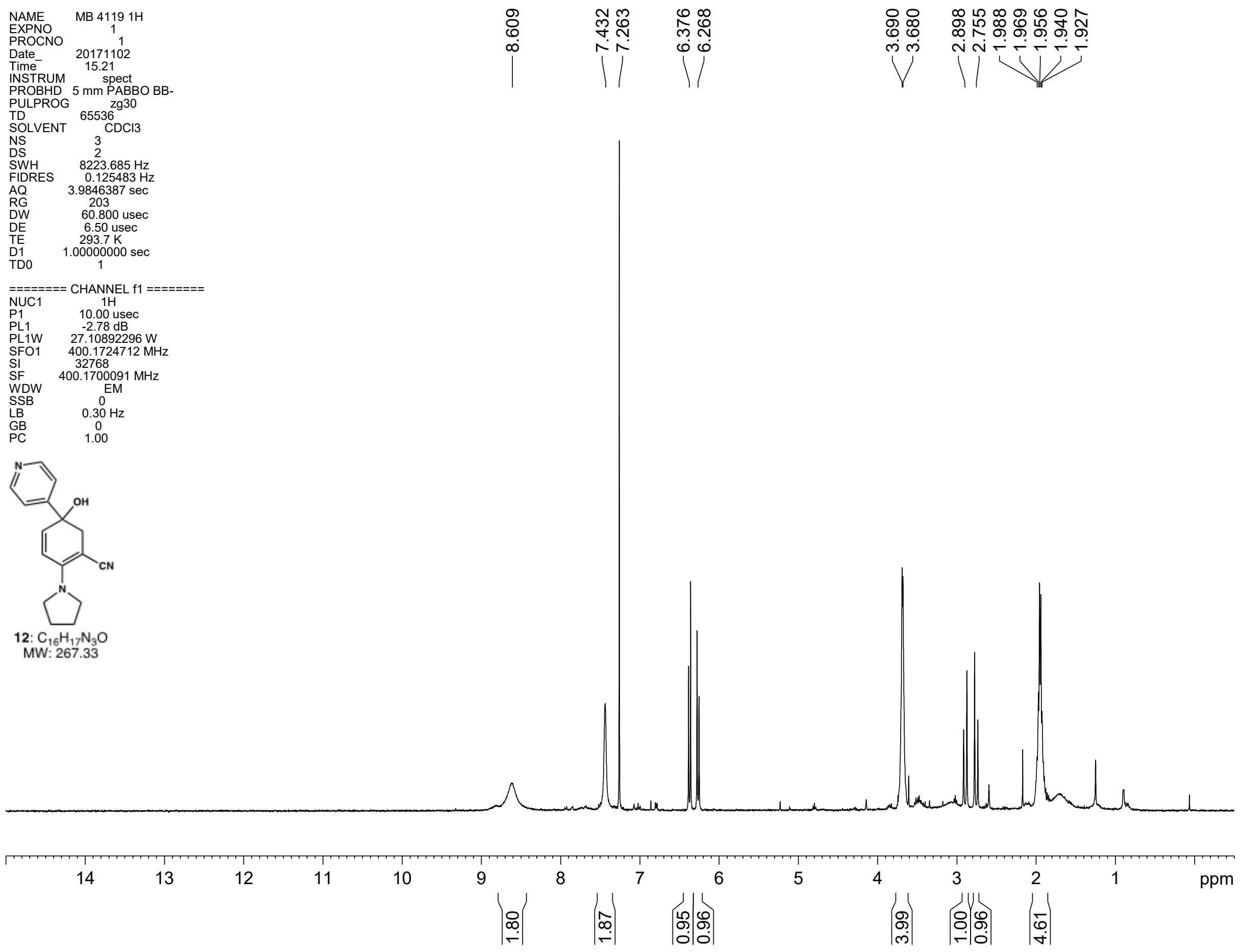




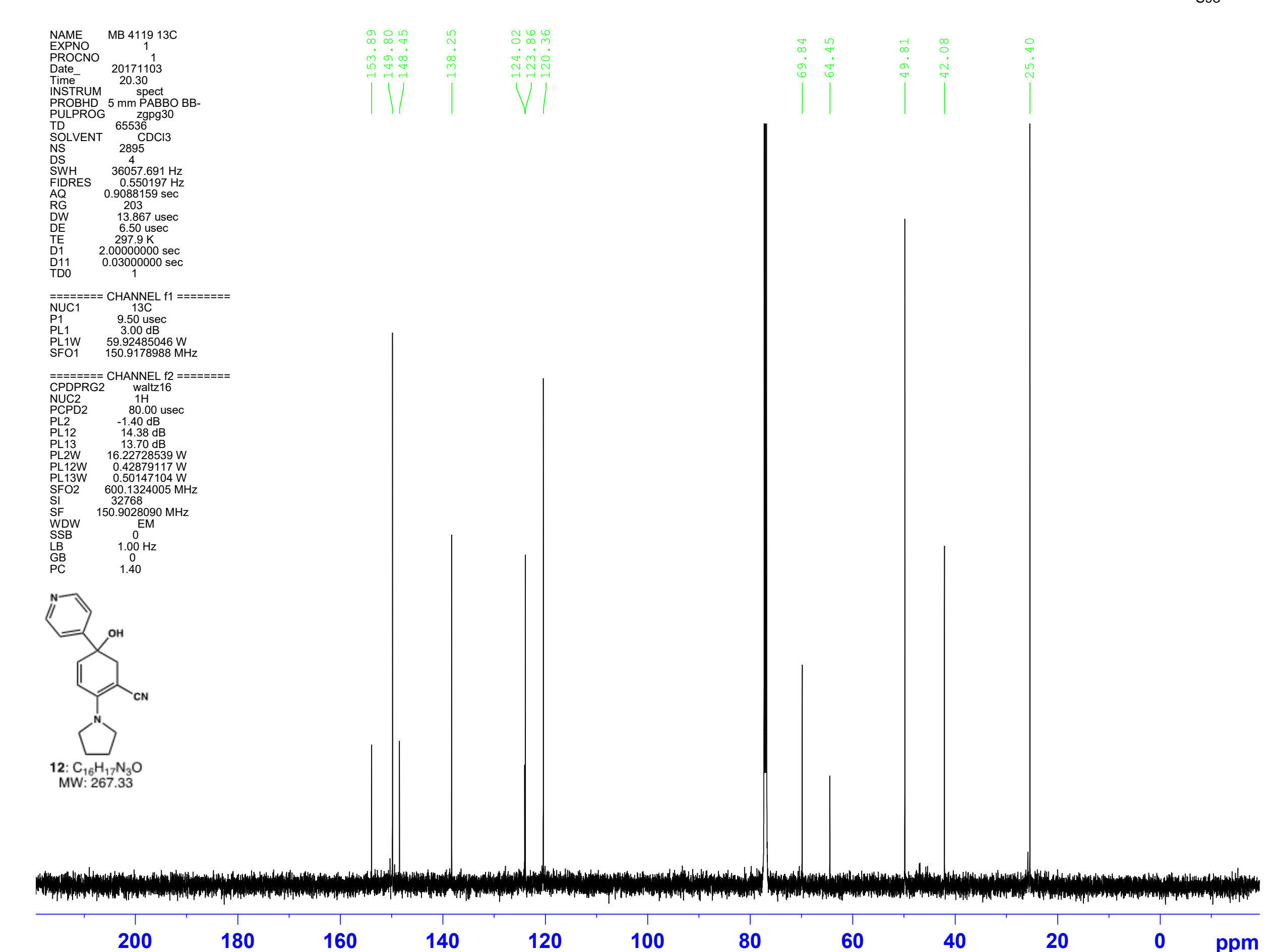




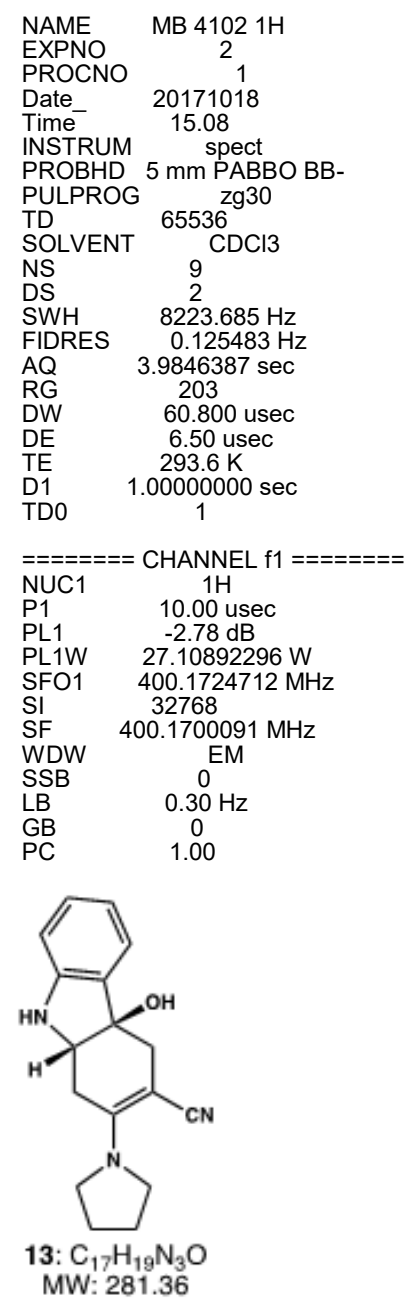

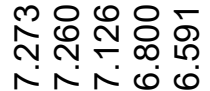

N $\hat{1}^{0}$

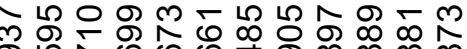
गें 1 N
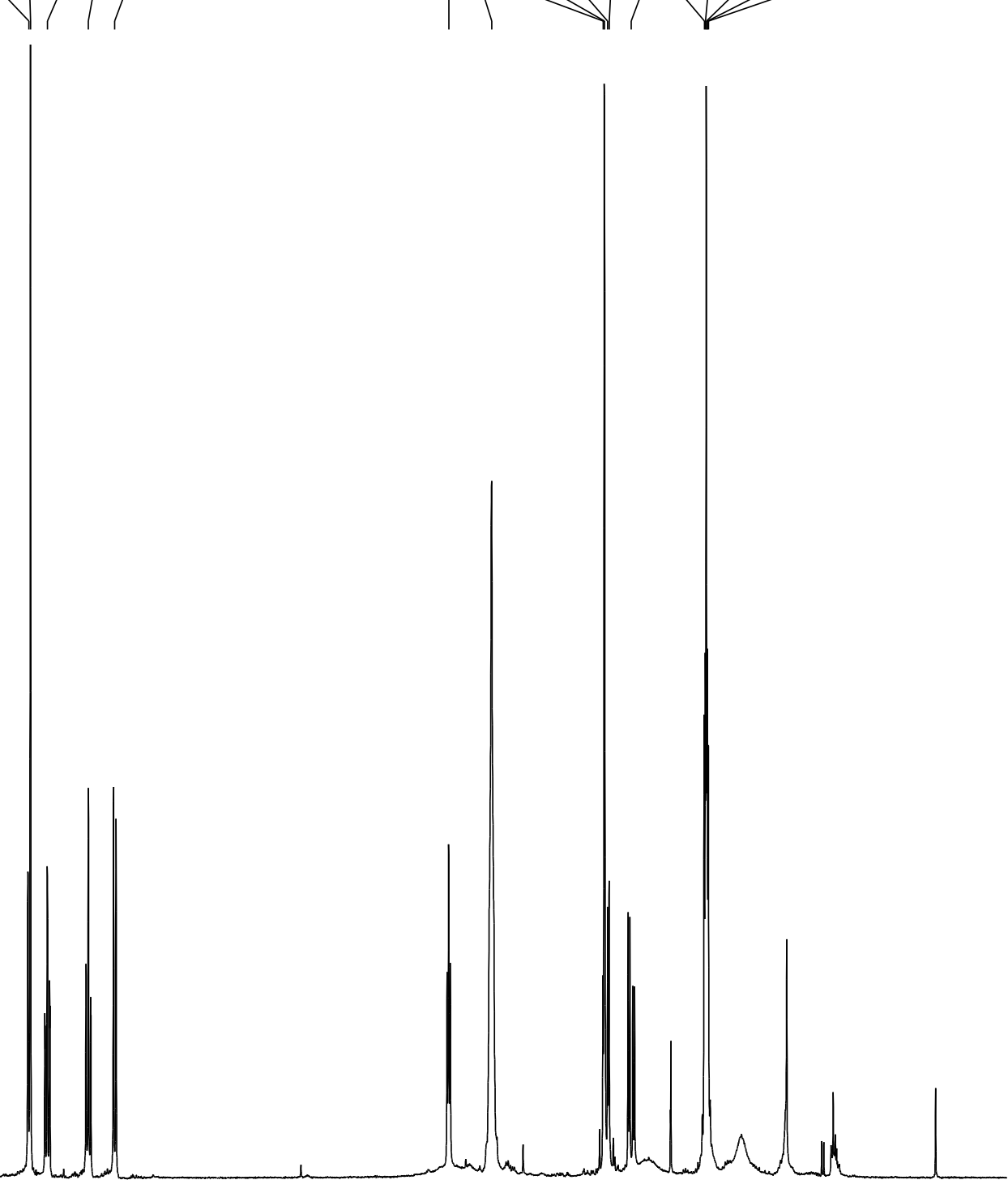

14

13

12

11

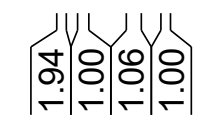

5

嘼唁

3

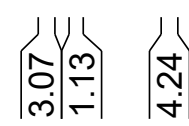




$$
1
$$




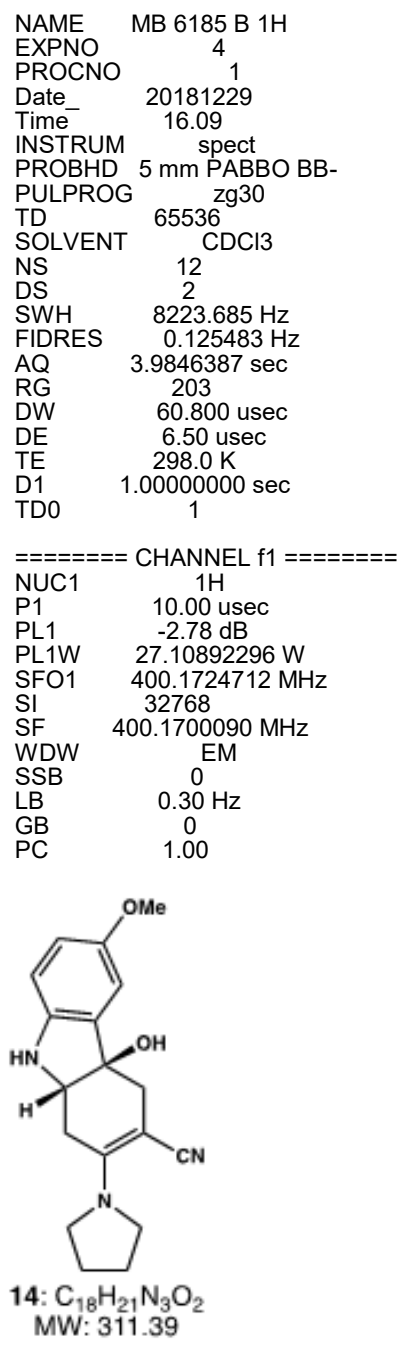

过品品品

त 00

111

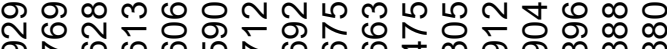
คin

INSTRUM 16.09

PABBO BB

SOLVENT CDCl3

DS

SWH $\quad 8223.685 \mathrm{~Hz}$

$0.125483 \mathrm{~Hz}$

203

$\mathrm{DE}$

$=======$ CHANNEL $\mathrm{f} 1$ =======

SFO1 $\quad 400.1724712 \mathrm{MHz}$

SF $\quad 400.1700090 \mathrm{MHz}$

WSB

LB

$0.30 \mathrm{~Hz}$ 

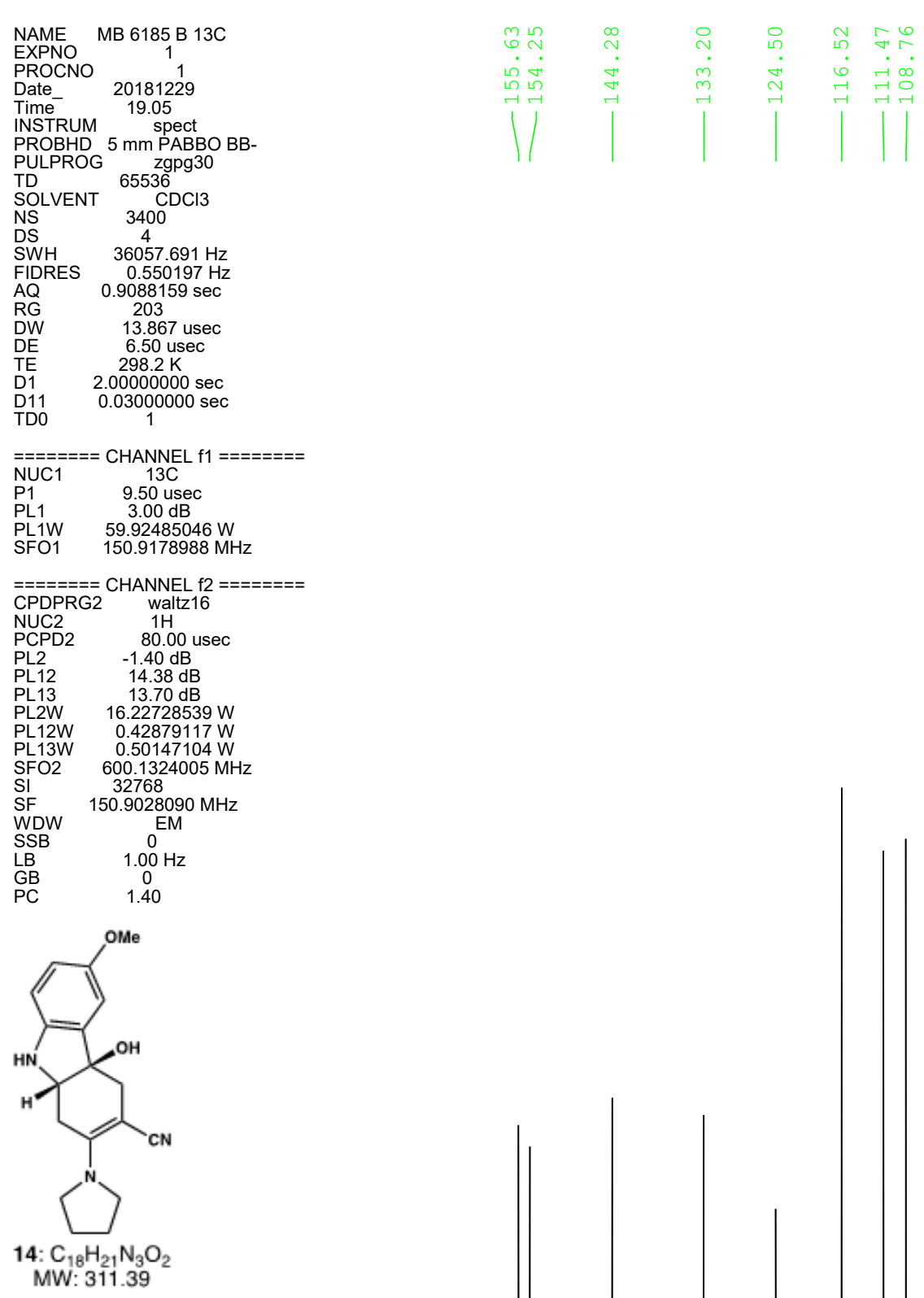

PULROG $5 \mathrm{~mm}$ PABBO BB-

TD 65536 g

NS $\quad 3400$

SWHES

$\mathrm{AQ} \quad 0.9088159 \mathrm{sec}$

DW $\quad 6.807$ usec

D1 $2.00000000 \mathrm{sec}$

1

$=======$ CHANNEL $\mathrm{f}$
NUC1

PL1 $\quad 3.00 \mathrm{~dB}$

SFO1 $150.9178988 \mathrm{MHz}$

$=======\mathrm{CHANNEL}$ f

PCPD2 $\quad 80.00$

PL12W $\quad 0.2285117 \mathrm{~W}$

$0.50147104 \mathrm{~W}$

SF $\quad 150.9028090 \mathrm{MHz}$

$\begin{array}{lr}\text { SSB } & 0 \\ \text { LB } & 1.00 \mathrm{~Hz}\end{array}$

OMe

MW: 311.39

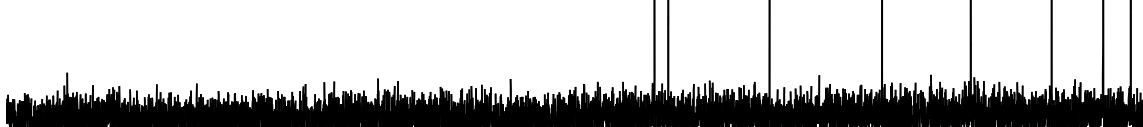

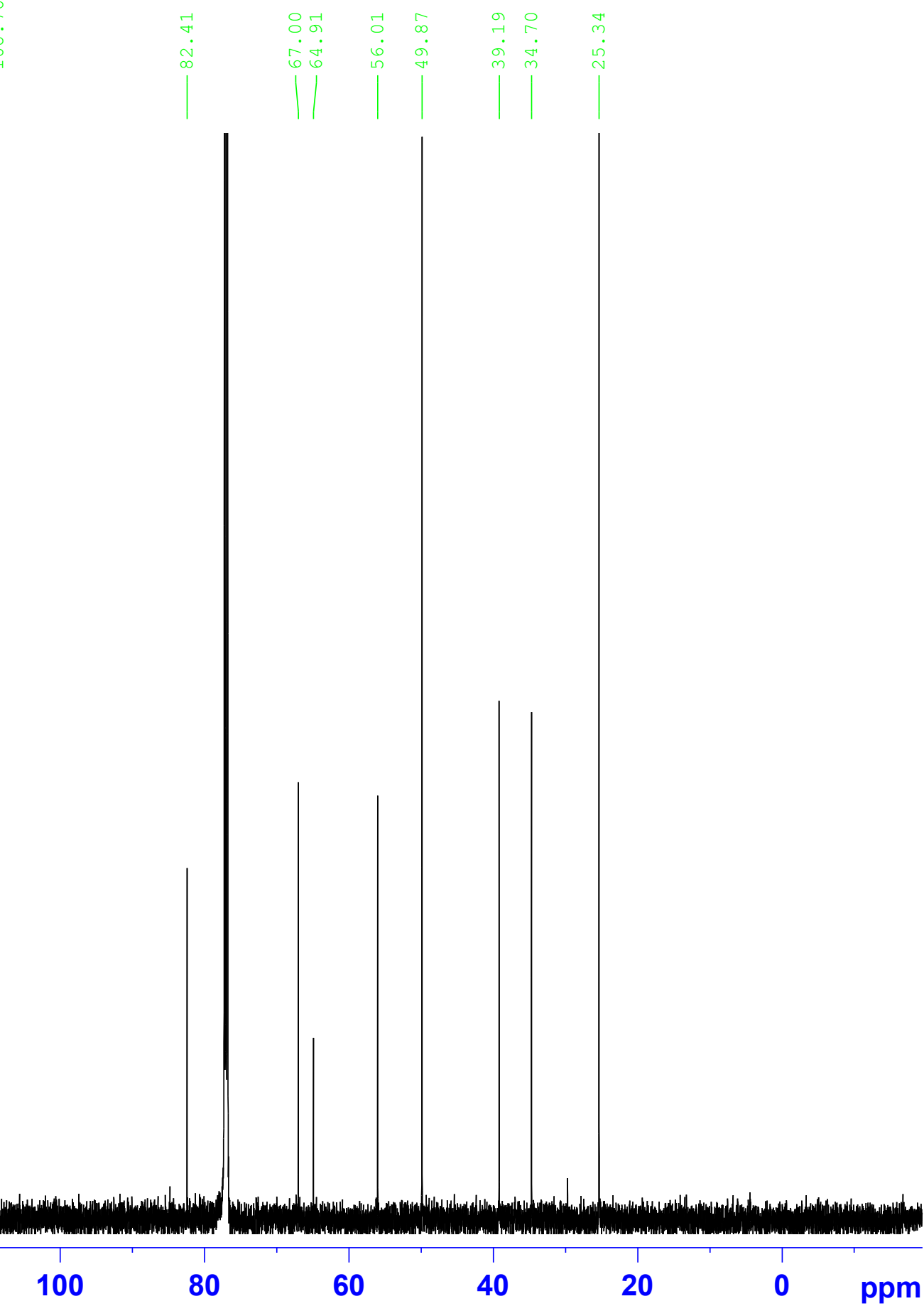



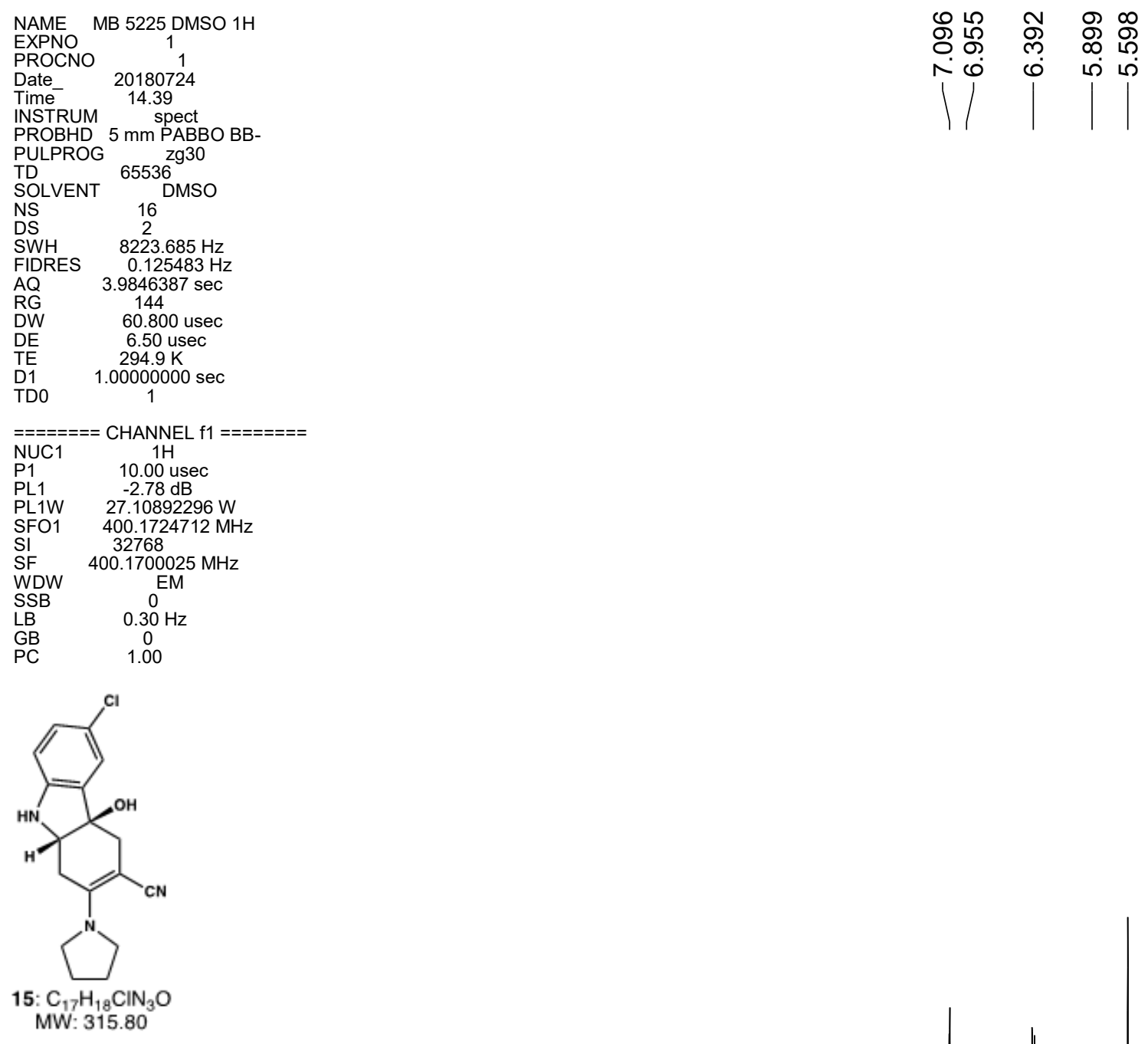

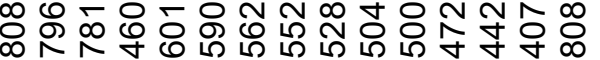

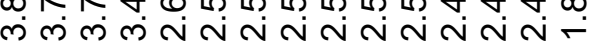

PULPROG 2930

SOLVENT

SWH $\quad 8223.685 \mathrm{~Hz}$

$0.125483 \mathrm{~Hz}$

144

DE

1.00000000

$=======$ CHANNEL $f 1=======$

SFO1 $\quad 400.1724712 \mathrm{MHz}$

SF $\quad 400.1700025 \mathrm{MHz}$

SSB 0

$\stackrel{L B}{G B} \quad 0.30 \mathrm{~Hz}$

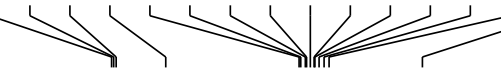

9

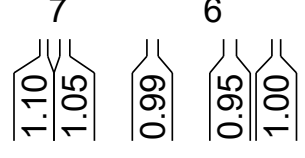

5

4 余|

3

2

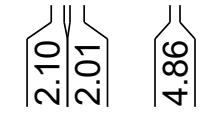

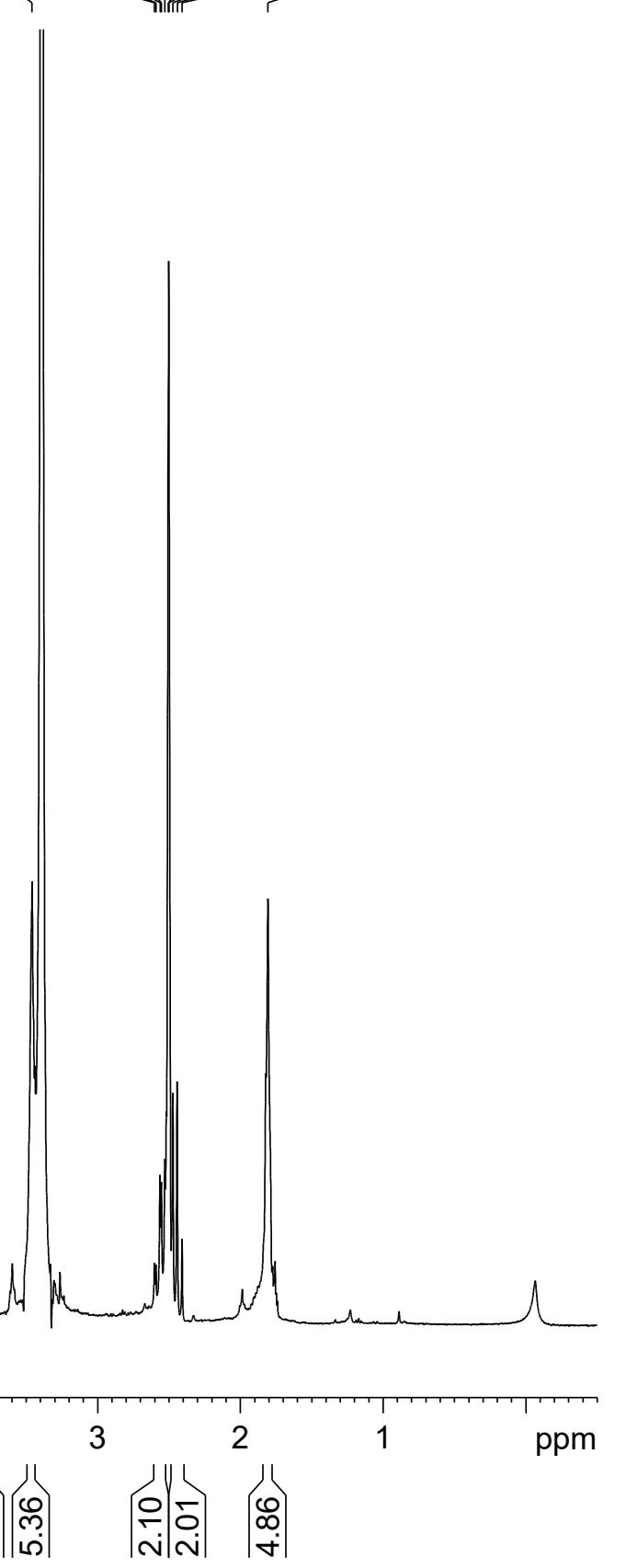



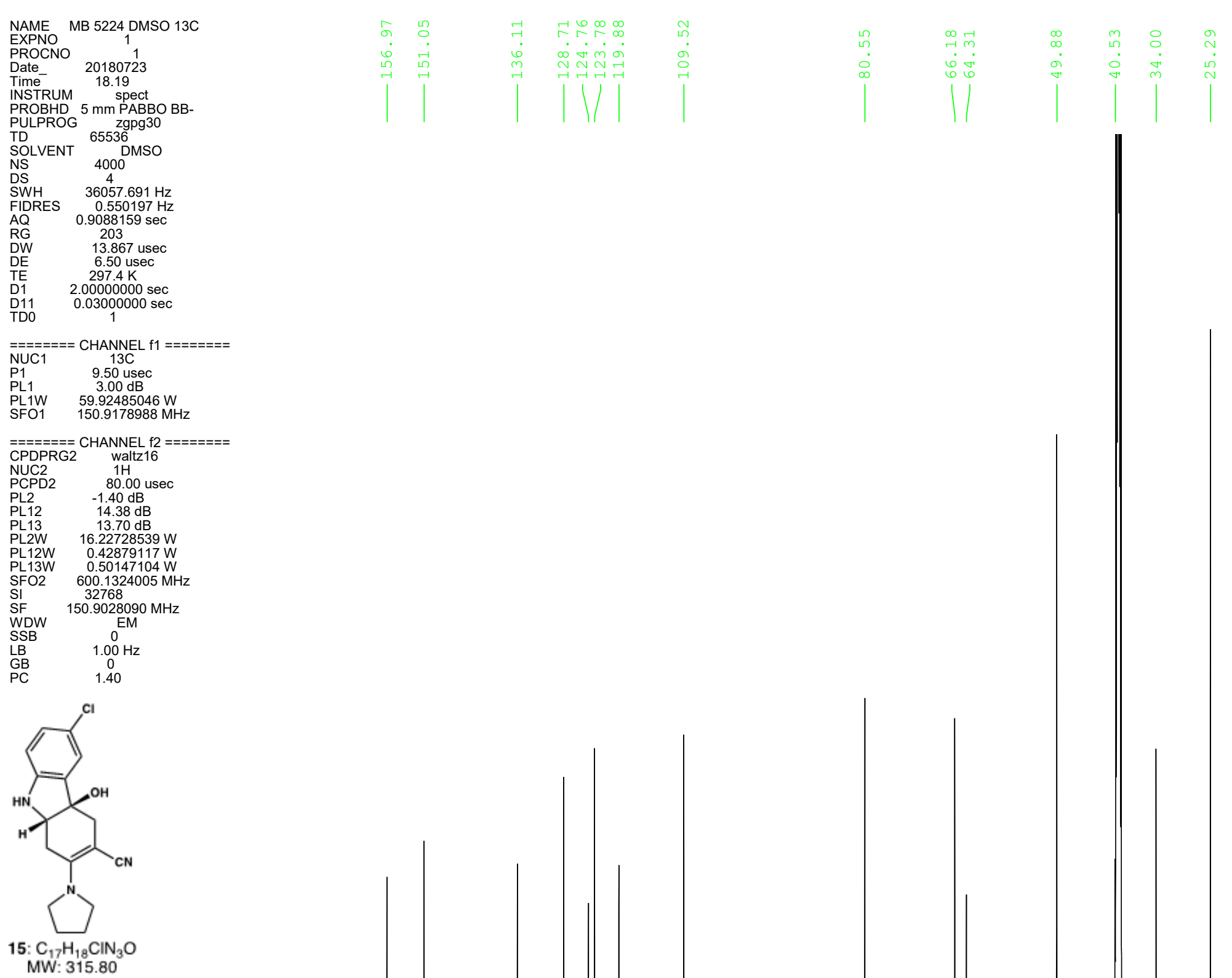

PULPROG zgpg30

SOLVENT $\quad$ DMSO

NS 4000

SWH $\quad 36057.691 \mathrm{~Hz}$

$\mathrm{AQ} \quad 0.9088159 \mathrm{sec}$

DE $\quad 6.867$ usec

D11 $0.03000000 \mathrm{sec}$

$========$ CHANNEL $\mathrm{f} 1$
NUC1

PL1 $\quad 3.00 \mathrm{~dB}$

SFO1 $150.9178988 \mathrm{MHz}$

$=======$ CHANNEL

$\begin{array}{ll}\text { NUCPD2 } & \text { 1H } \\ \text { PCO }\end{array}$

$28090 \mathrm{MHz}$

$\begin{array}{lc}\mathrm{SSB} & 0 \\ \mathrm{LB} & 1.00 \mathrm{~Hz}\end{array}$

MW: 315.80

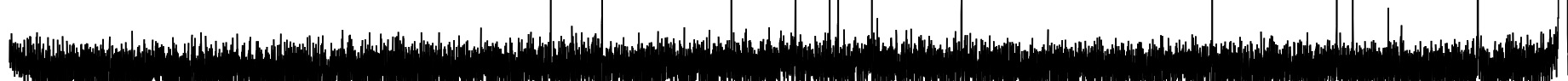

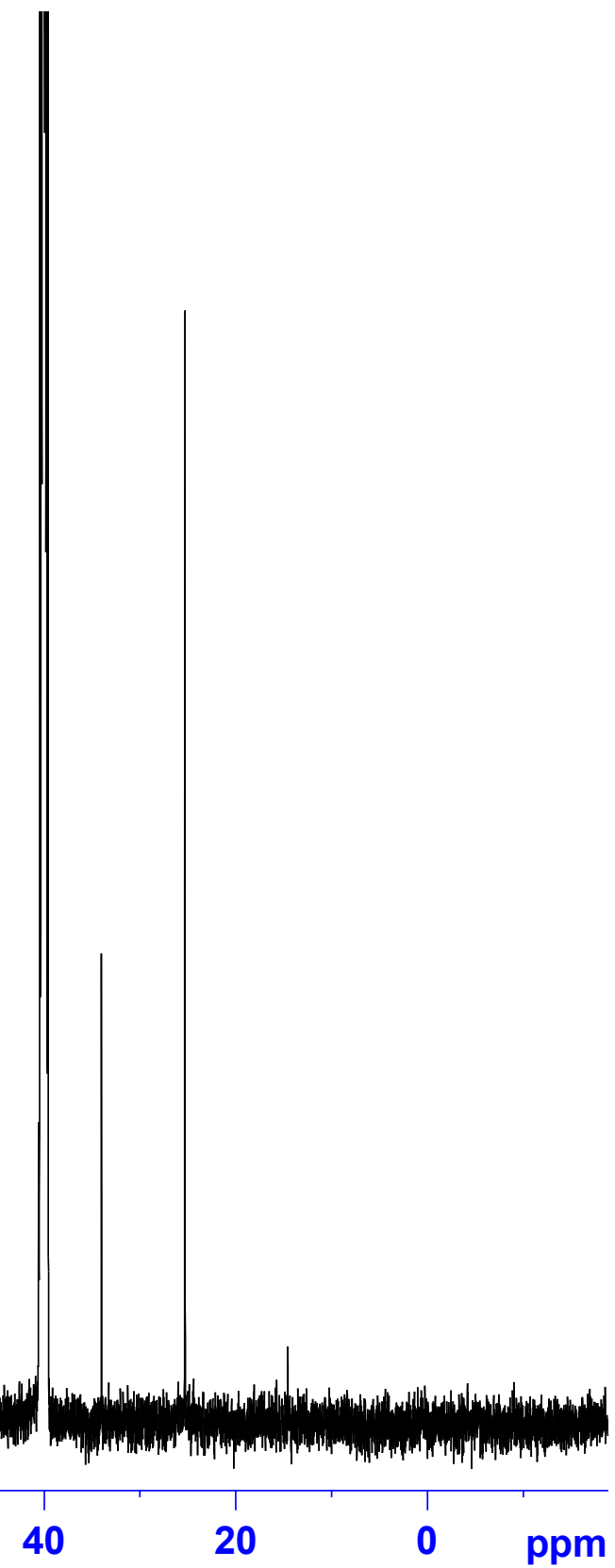



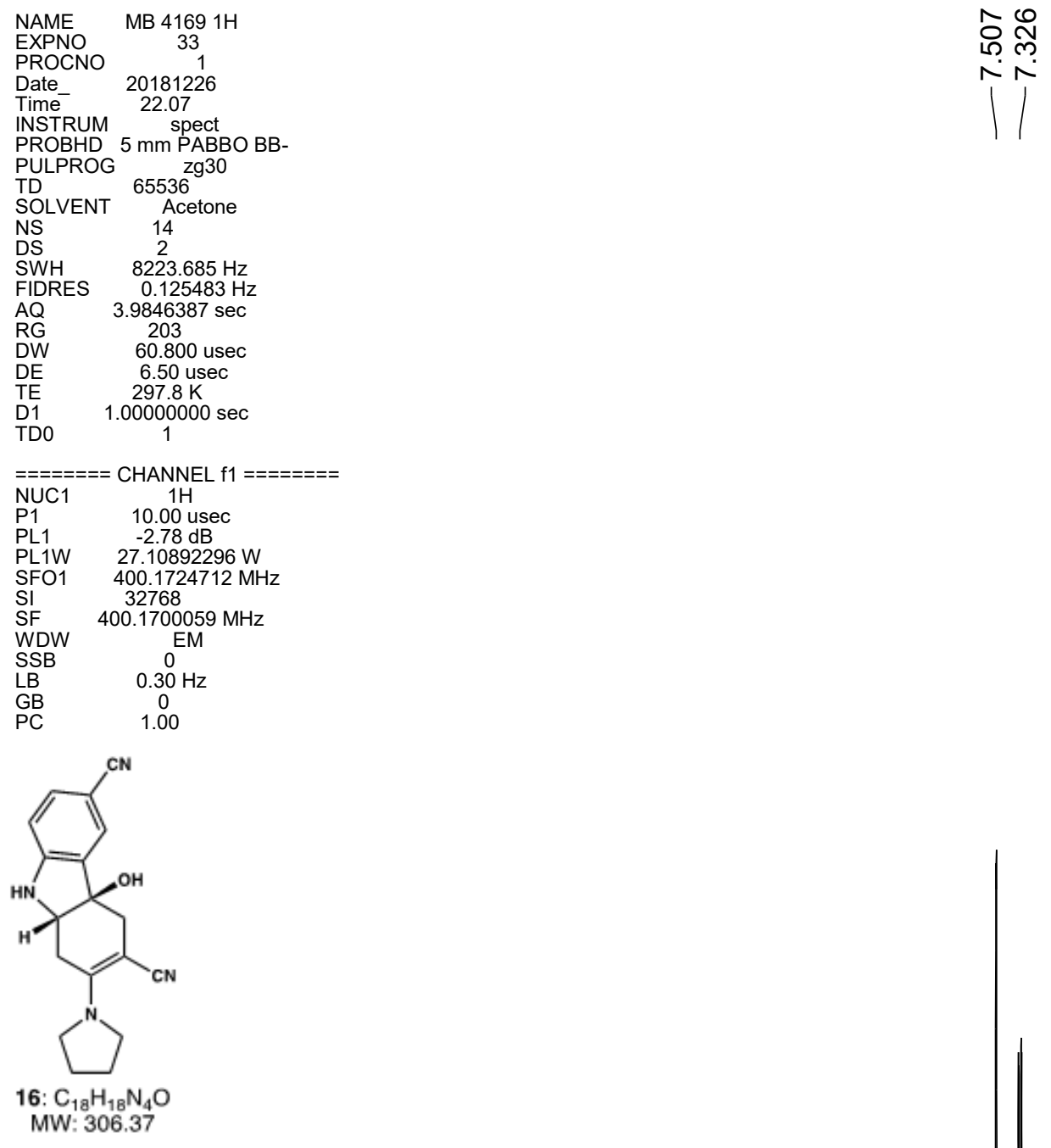

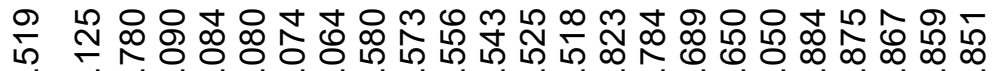

0

PROC

1

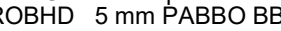

SWH

$1.0000000 \mathrm{sec}$

$========$ CHANNEL $\mathrm{f} 1$ ========

SFO1 $400.1724712 \mathrm{MH}$

SF $\quad 400.1700059 \mathrm{MHz}$

WDW $\quad$ EM

$\mathrm{LB} \quad 0.30 \mathrm{~Hz}$
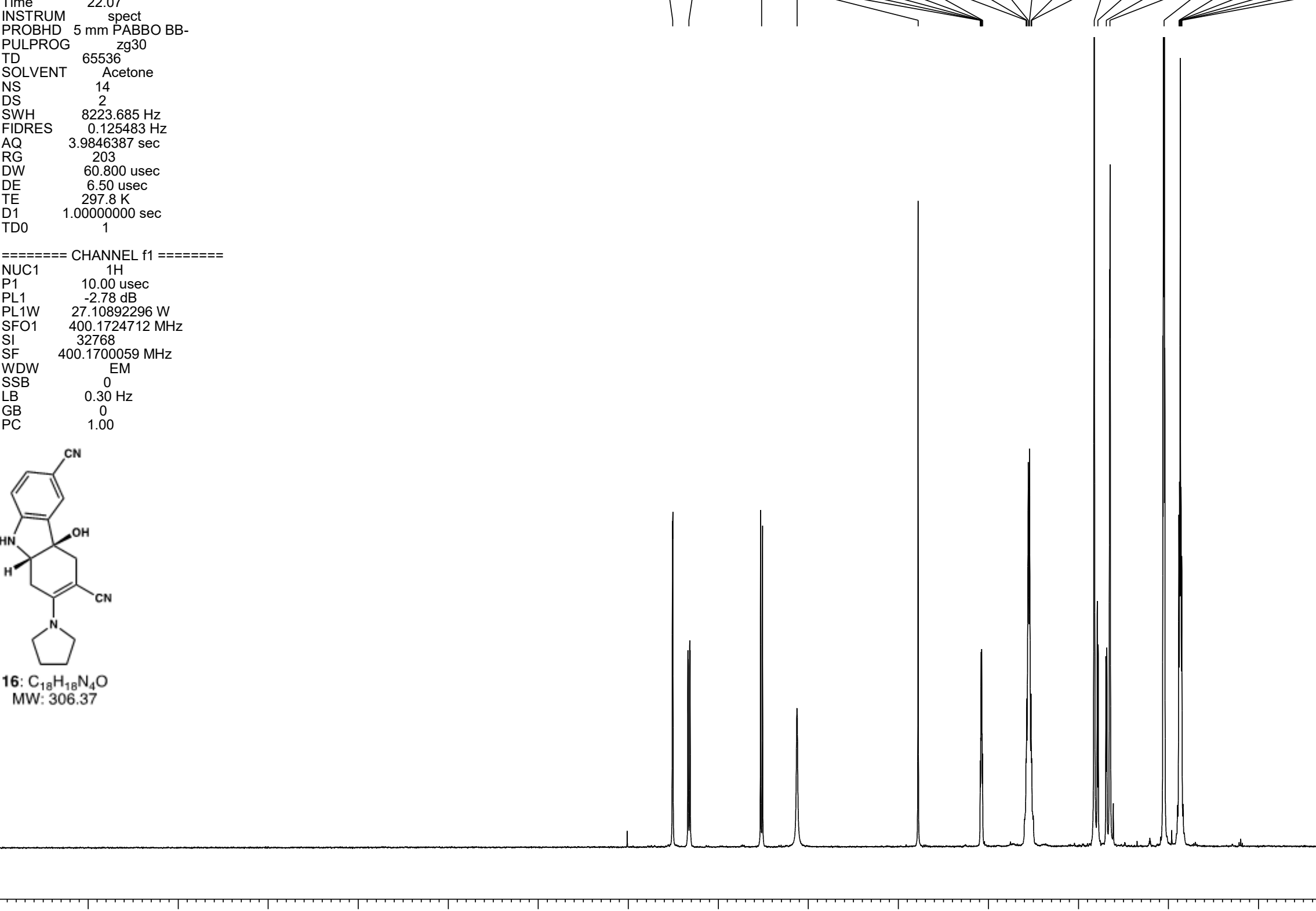

14

13

12

11

10

9

8 (4)

7 â.

6

5

4

3

2

1

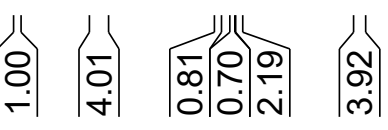



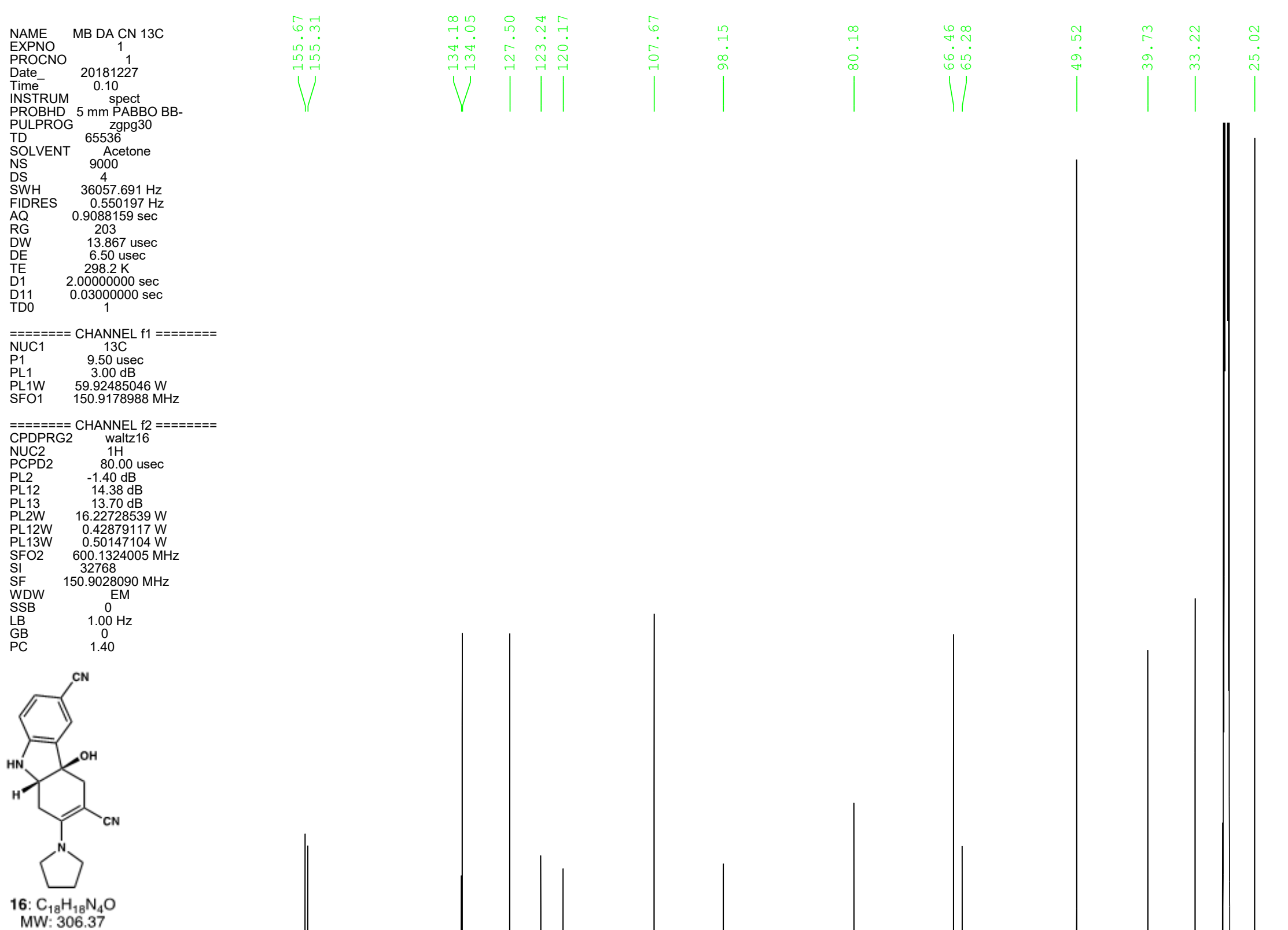

$5 \mathrm{~mm}$ PABBO

TD 65536

NS $\quad 9000$

DWHES

$\begin{array}{ll}\mathrm{RG} & 203 \\ \mathrm{DW} & 13.867 \mathrm{usec}\end{array}$

TE $298.2 \mathrm{~K}$

D11 $\quad 2.0000000 \mathrm{sec}$

$=======$ CHANNEL $f 1$ =======

MW: 306.37
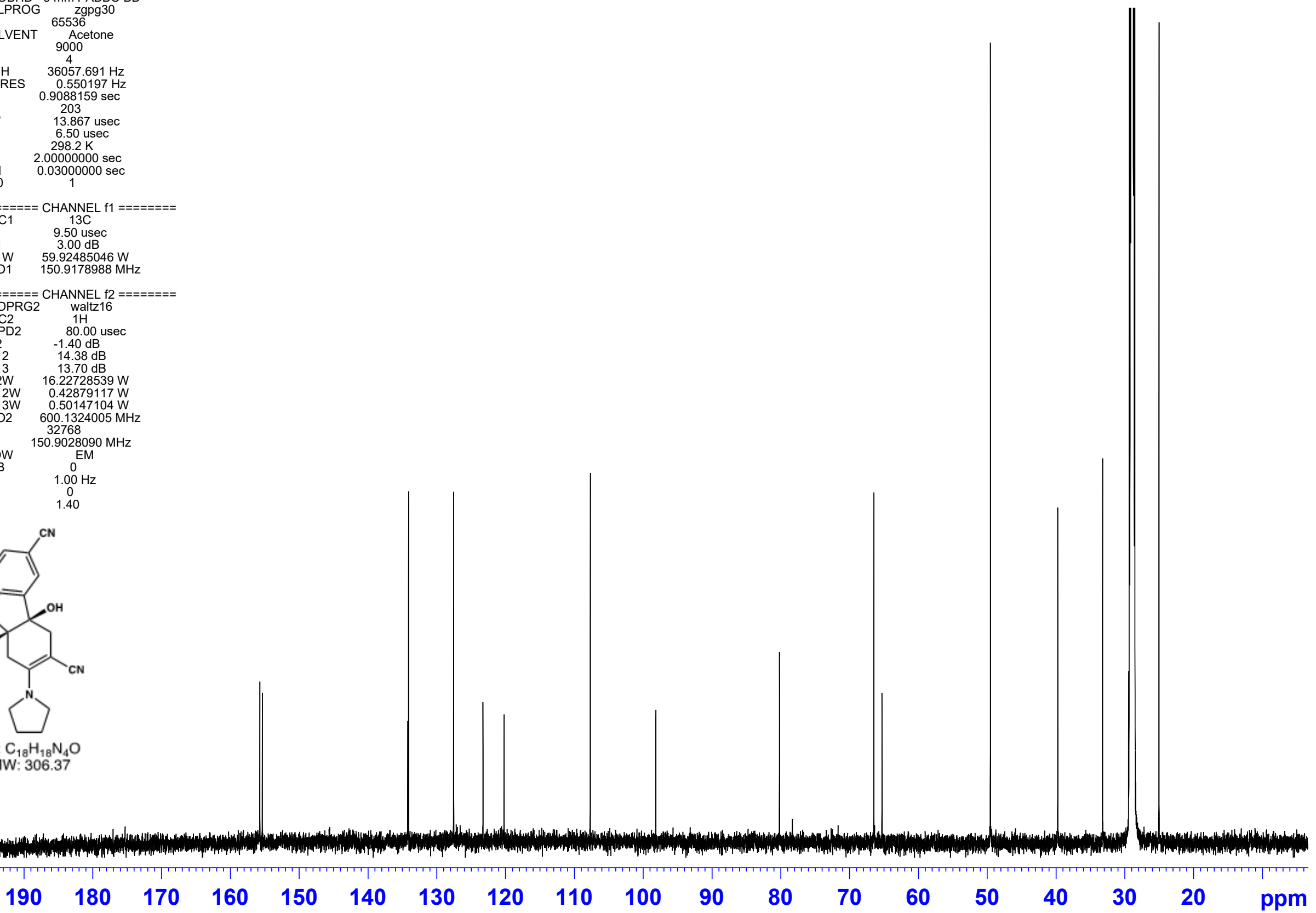


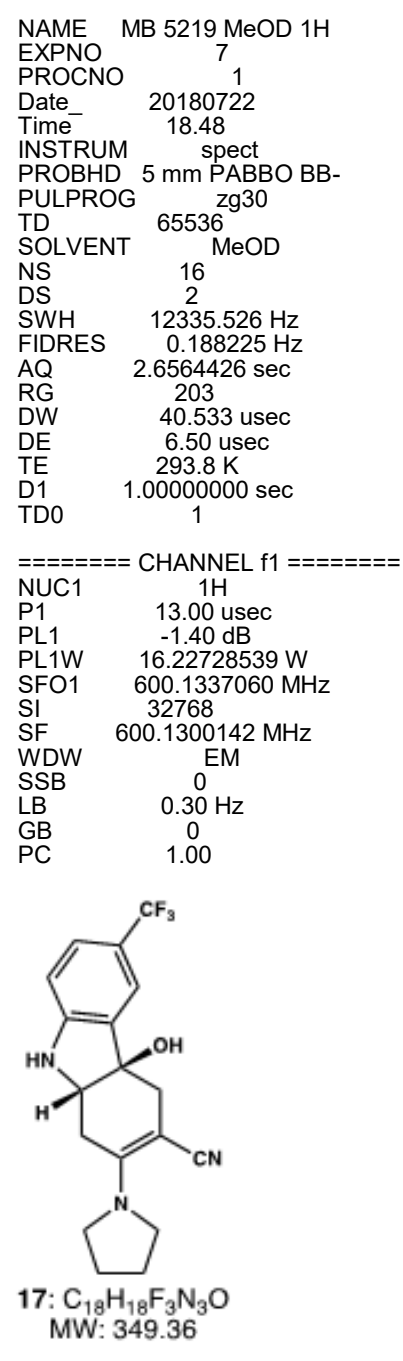

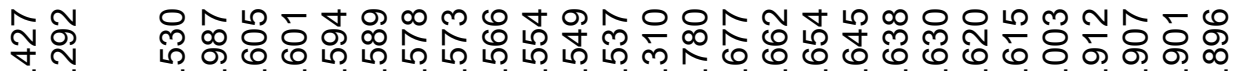
ต $1 / 1+1,1<1<1$

BBO BB-

SOLVENT MeOD

$=======$ CHANNEL $f 1$ =======

$-1.40 \mathrm{~dB}$

$\begin{array}{ll}\text { PL1W } & 16.22728539 \mathrm{~W} \\ \text { SFO1 } & 600.1337060 \mathrm{MH}\end{array}$

SI $\quad 32768$

WDW

$0.30 \mathrm{H}$

1.00

MW: 349.36
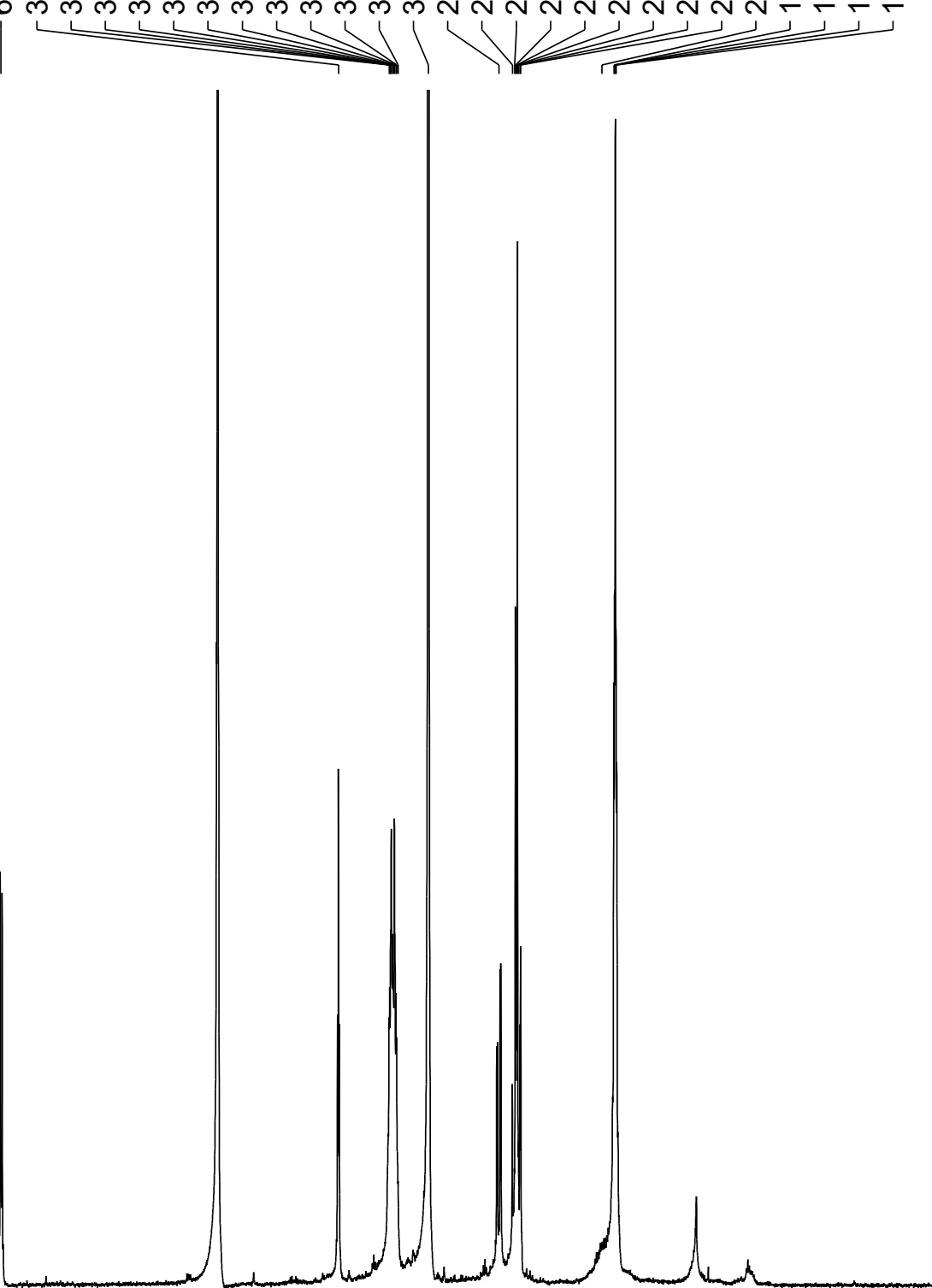

$14 \quad 13$

12

11

10

9

8

瞵堘

7
0
0
0
0

6

5

$\begin{array}{ll}4 & \\ 0 & 0 \\ 0 \\ 0 \\ 0\end{array} \mid$

3

2

1 


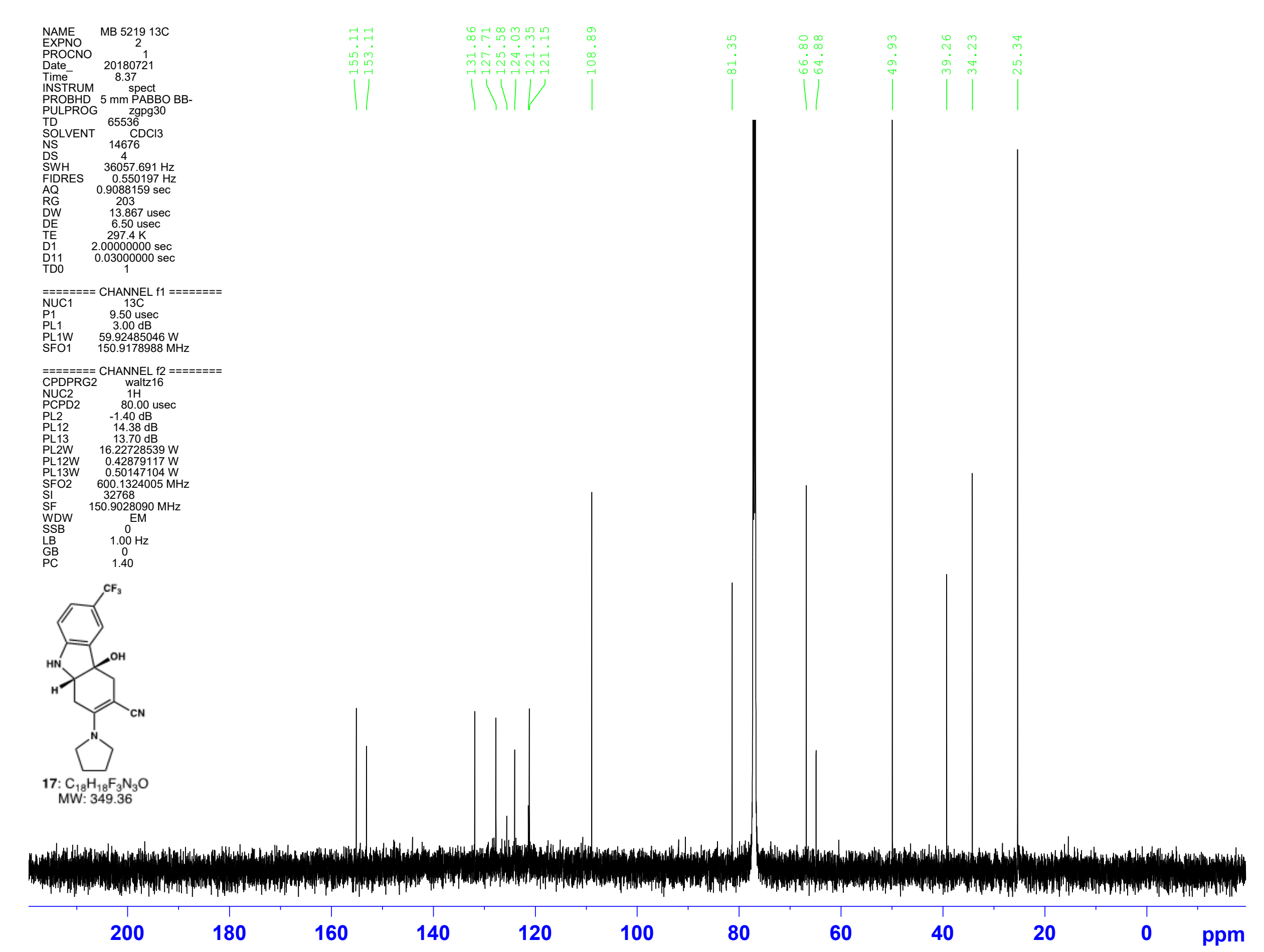




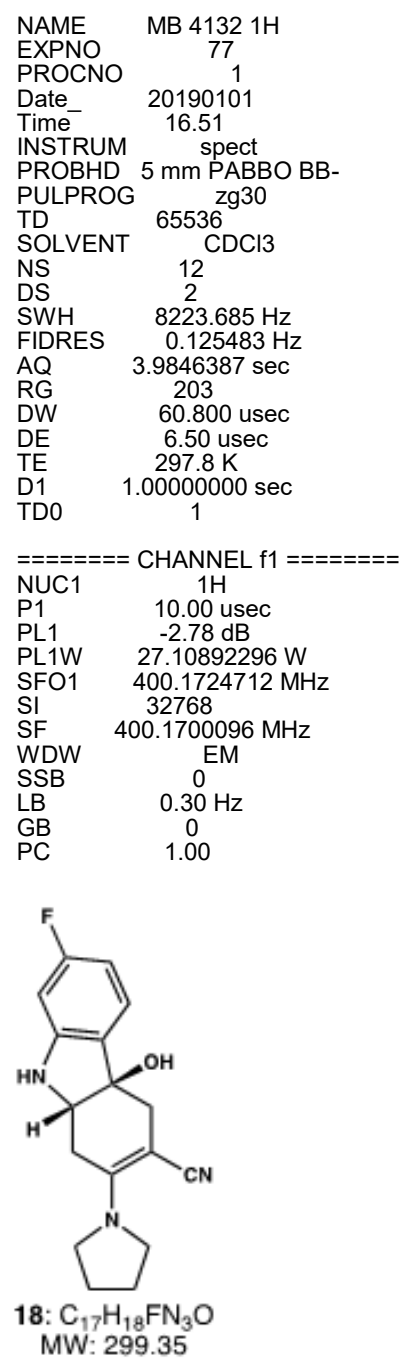

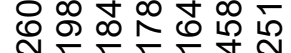

NNNNo

)

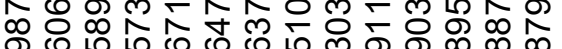

نंmin
$========$ CHANNEL $\mathrm{f} 1$ ========

SFO1 $400.1724712 \mathrm{MH}$

SF $\quad 400.1700096 \mathrm{MHz}$

WDW $\quad$ EM
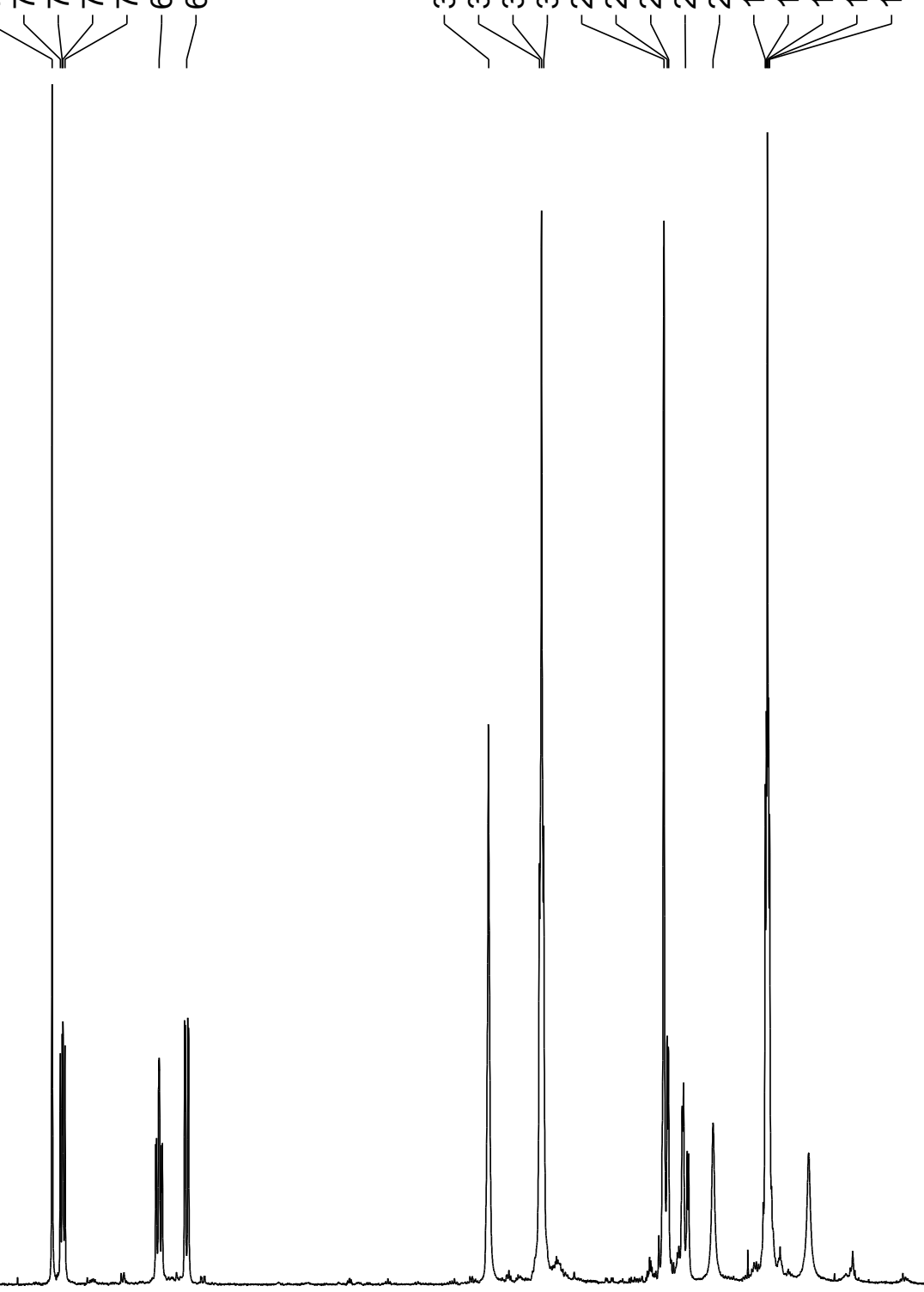

14 


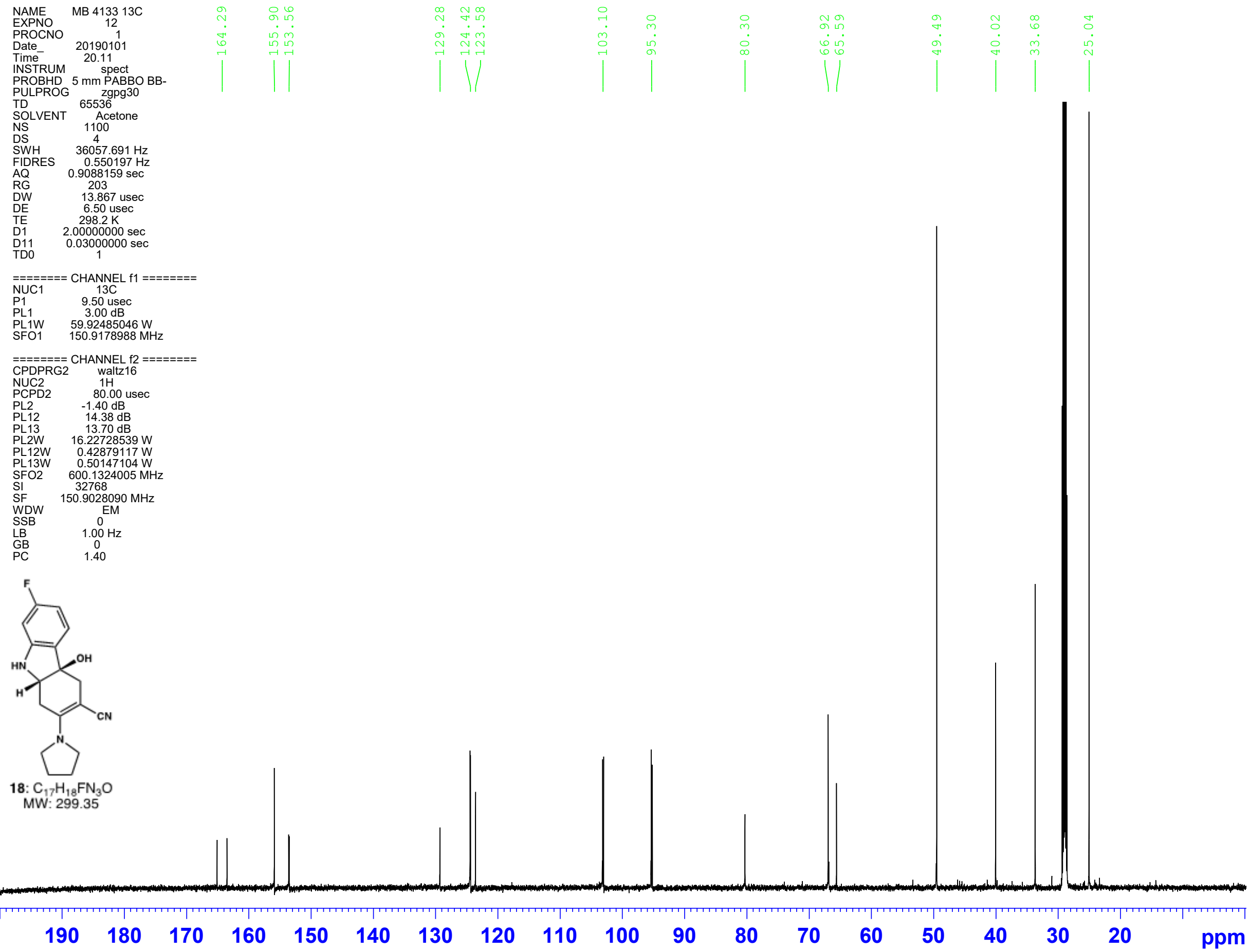




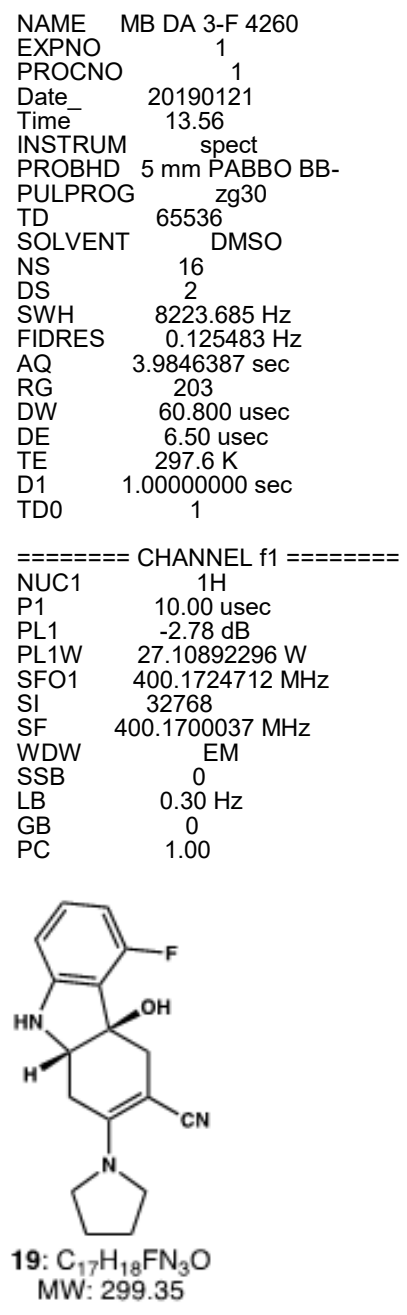

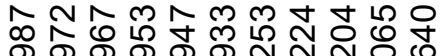
๑

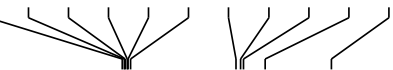

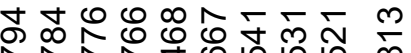

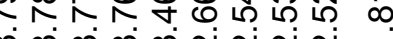

PROBHD 5 mmPABBO BB-

PULPROG zg30

SOLVENT

SWH

$=======$ CHANNEL $\mathrm{f} 1$ ========

PL1W $27.10892296 \mathrm{~W}$

SI $\quad 32768$

WDW $\quad$ EM

$.30 \mathrm{~Hz}$

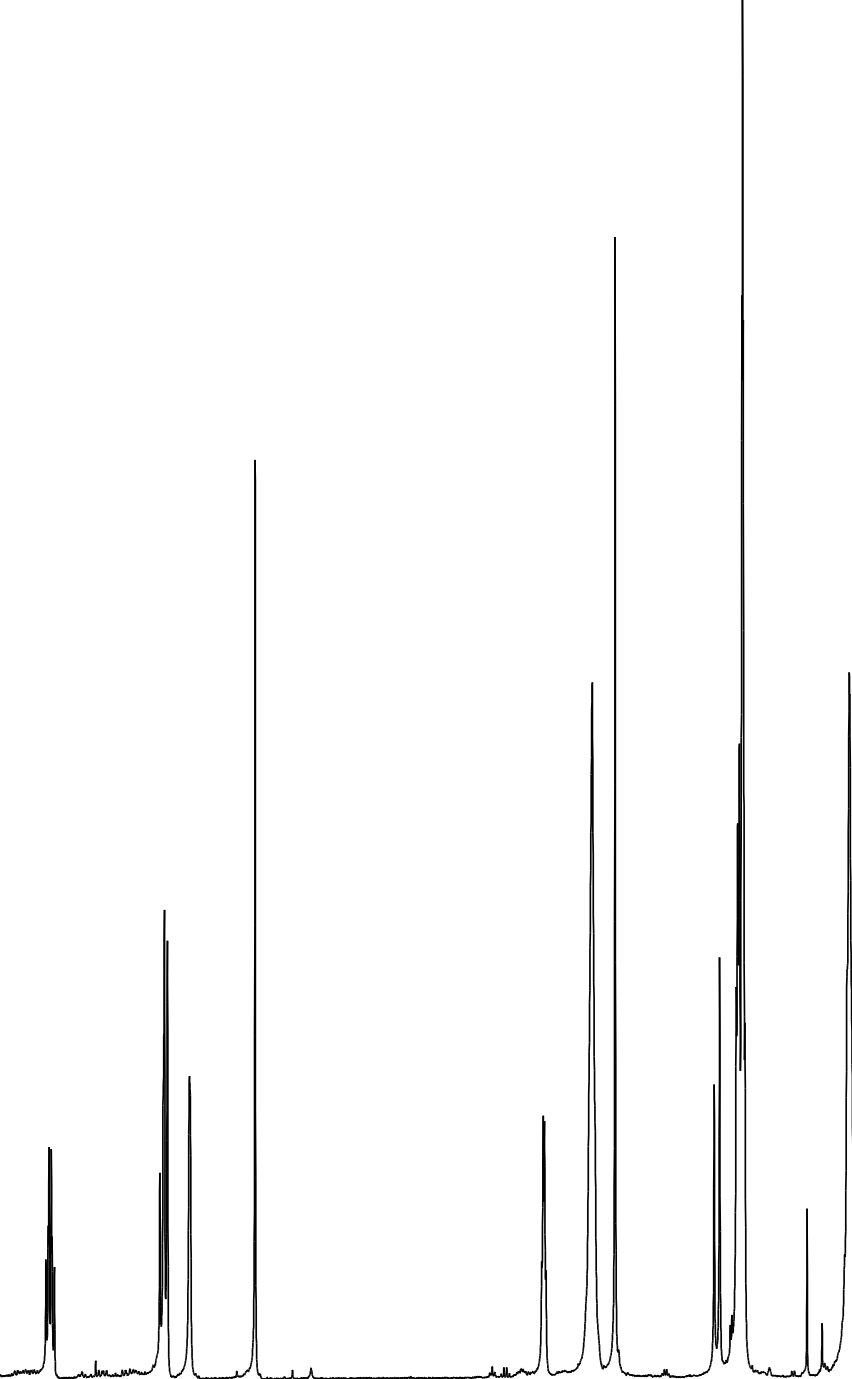

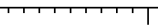



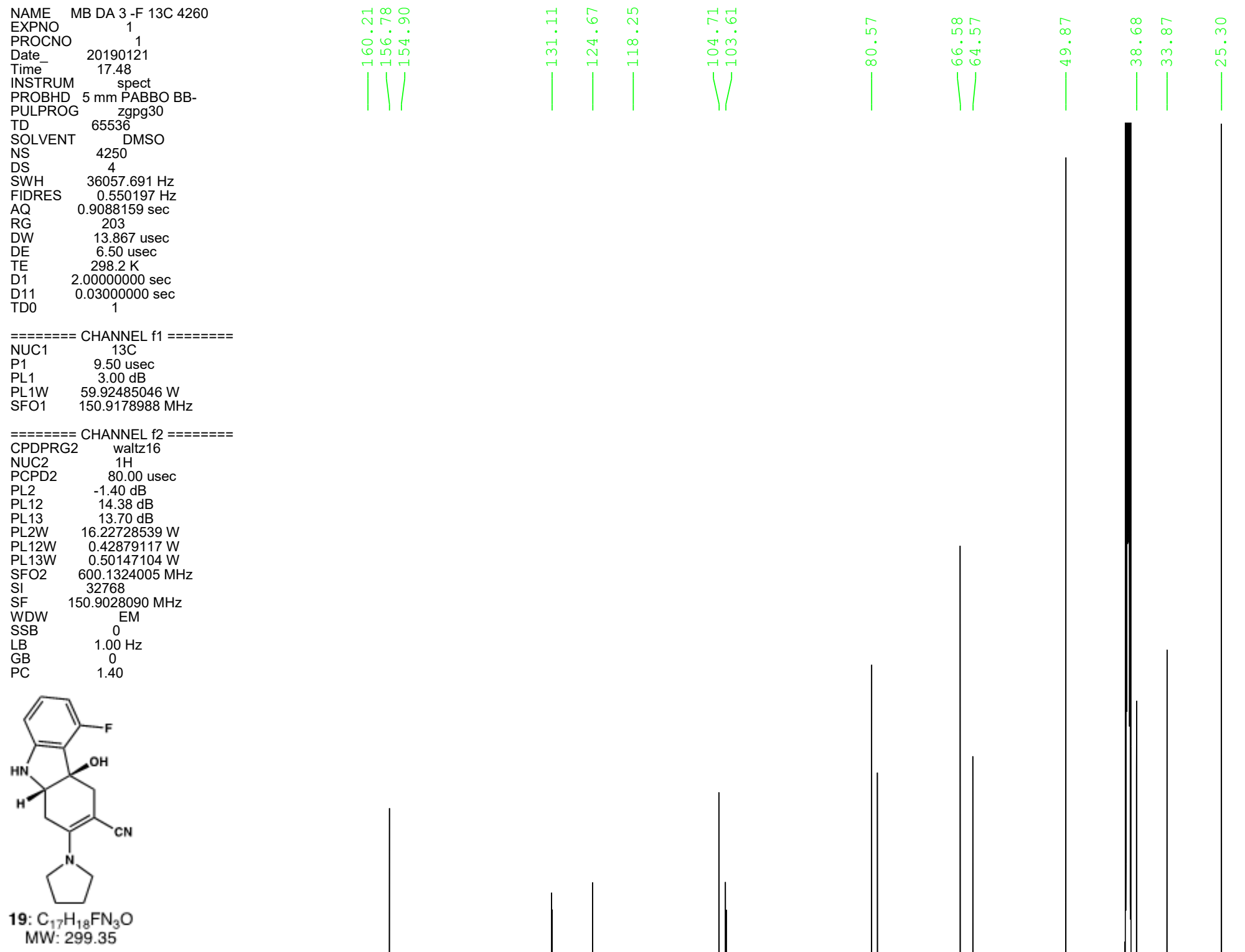

PULPROG

SOLVENT

NS $\quad 4250$

FWH $\quad 36057.691 \mathrm{~Hz}$

AQ $\quad 0.9088159 \mathrm{sec}$

$\begin{array}{ll}\text { DW } & 13.867 \text { usec } \\ \text { DE } & 6.50 \text { usec }\end{array}$

D1 $\quad 2.00000000 \mathrm{~s}$

TD0

$=======$ CHANNEL
NUC1 $13 \mathrm{C}$

PL1 $\quad 3.00 \mathrm{~dB}$

SFO1 $150.9178988 \mathrm{MHz}$

$=======$ CHANNEL f 2

$\begin{array}{lc}\text { NUC2 } & 1 \mathrm{H} \\ \text { PCPD2 } & 80.00 \text { use }\end{array}$

$\begin{array}{ll}\text { PL12 } & 14.38 \mathrm{~dB}\end{array}$

PL12W $\quad 0.42879117$ W

$0.50147104 \mathrm{~W}$

SI $\quad 32768$

$\begin{array}{ll}\text { WDW } & 150.9028090 \\ \text { SSB } & \text { EM }\end{array}$

$\begin{array}{lc}\text { LB } & 1.00 \\ \text { GB } & 0 \\ \text { PC } & 1.40\end{array}$

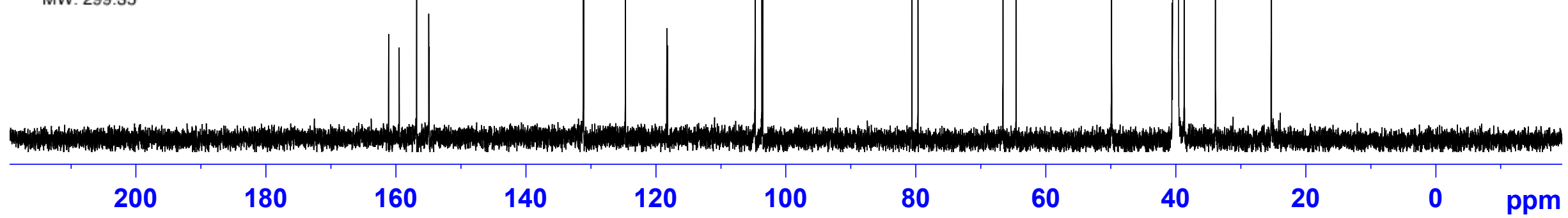




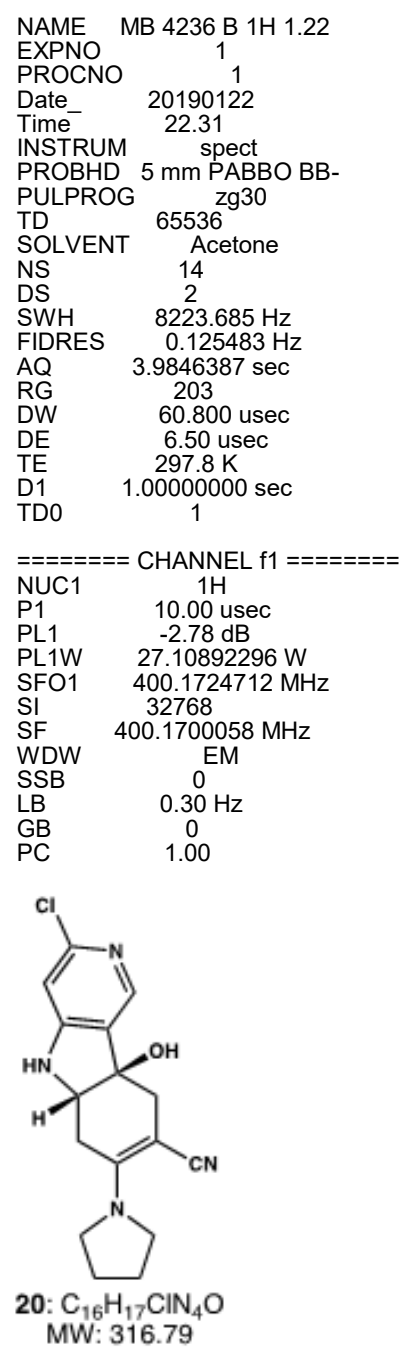

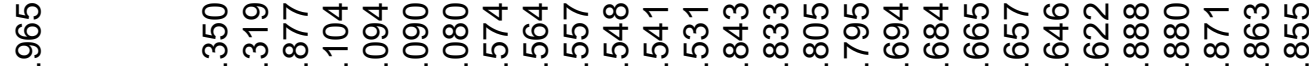
ஸें

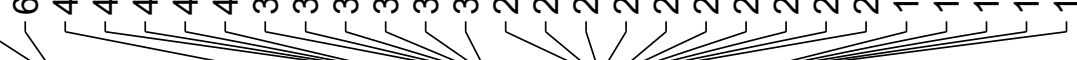

MW: 316.79

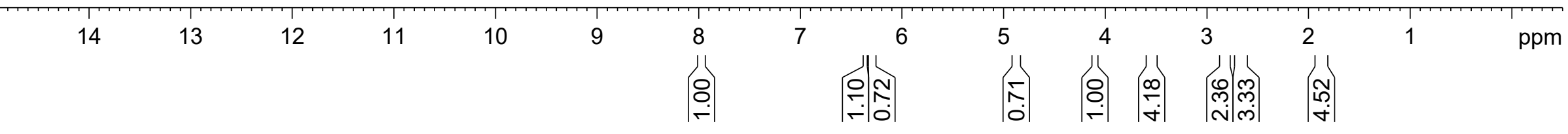




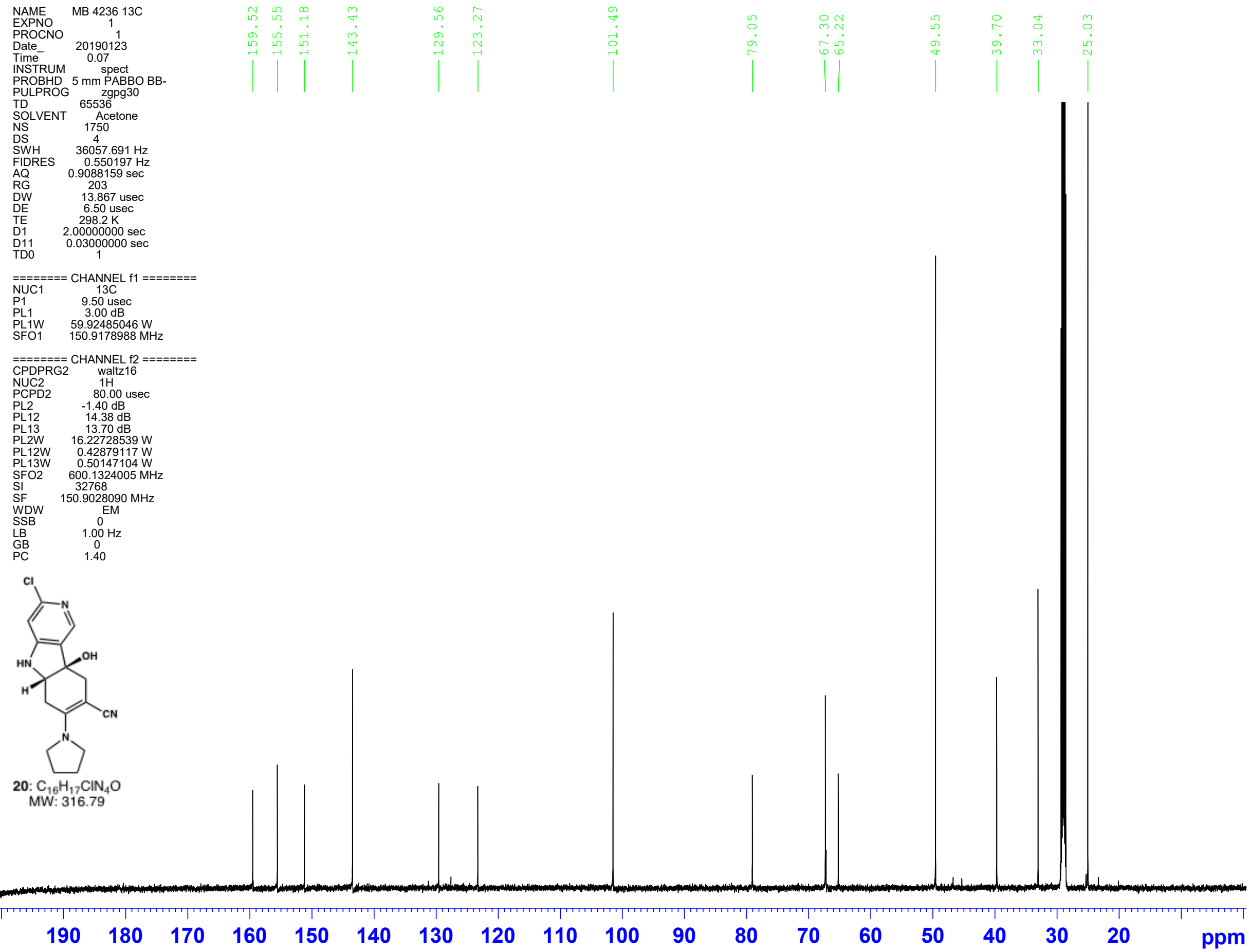




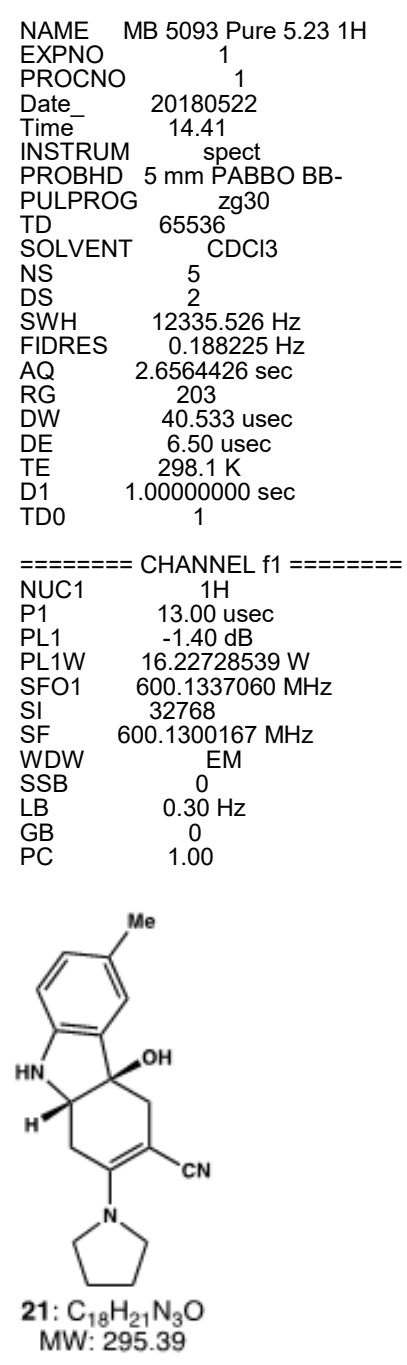

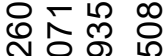

N

111

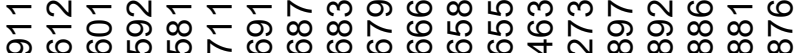

ต

m m m
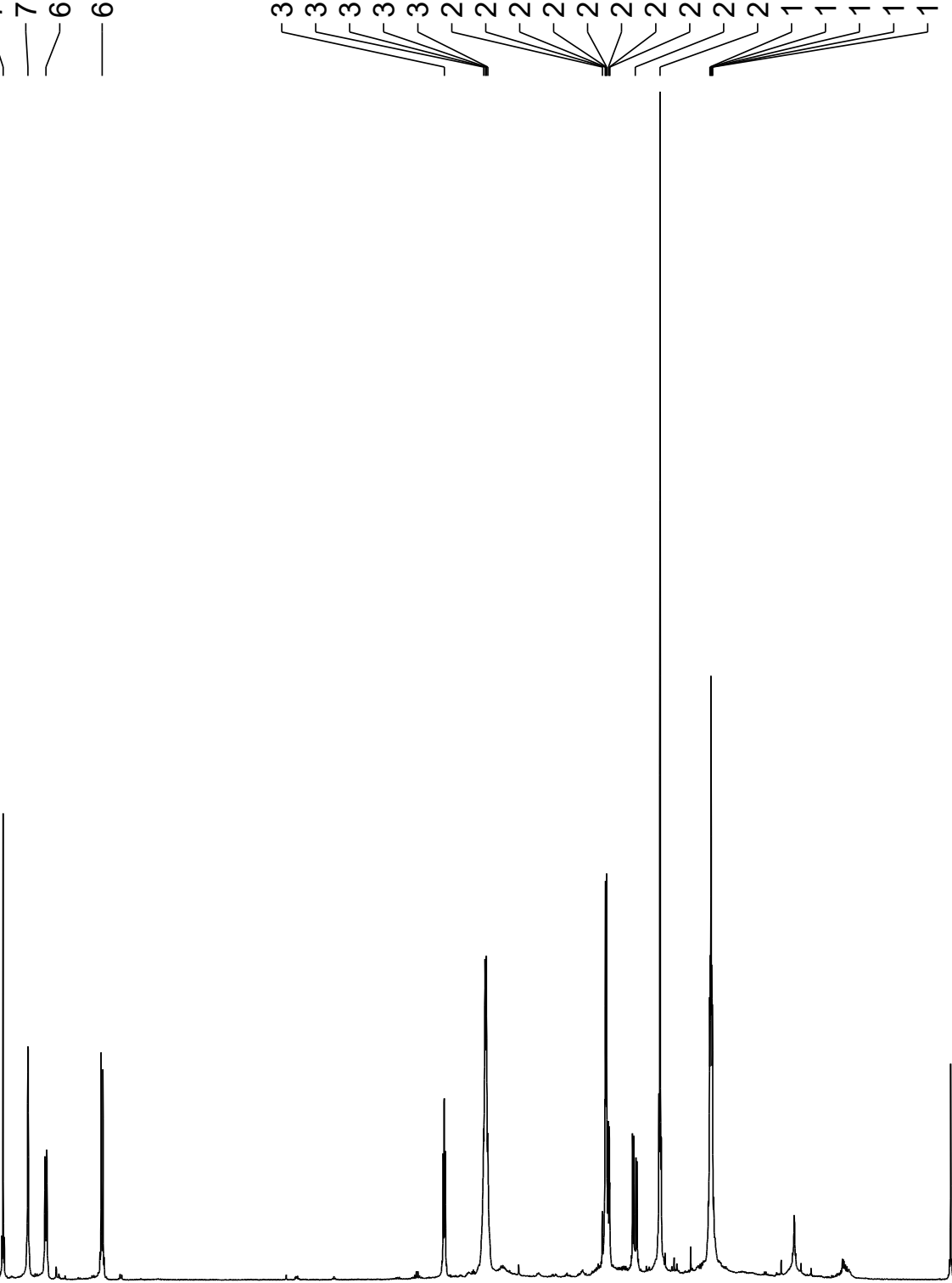

$12335.526 \mathrm{~Hz}$

$0.188225 \mathrm{~Hz}$

203

DE $\quad 6.50$ usec

NUC1 $1 \mathrm{H}$

32768

WDW $600.1300167 \mathrm{MHz}$

SSB

LB

$0.30 \mathrm{~Hz}$

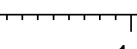

14

13

12

11

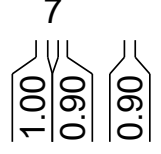

6

5

\begin{tabular}{l|l}
4 \\
$m$ \\
0 \\
0
\end{tabular}$\left|\begin{array}{l}10 \\
0\end{array}\right|$

3

2

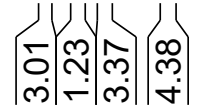




$$
1
$$




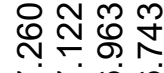
111

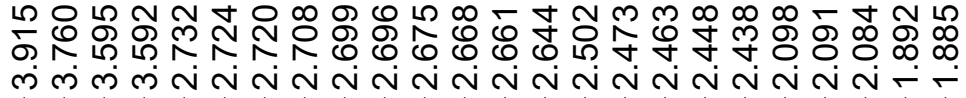

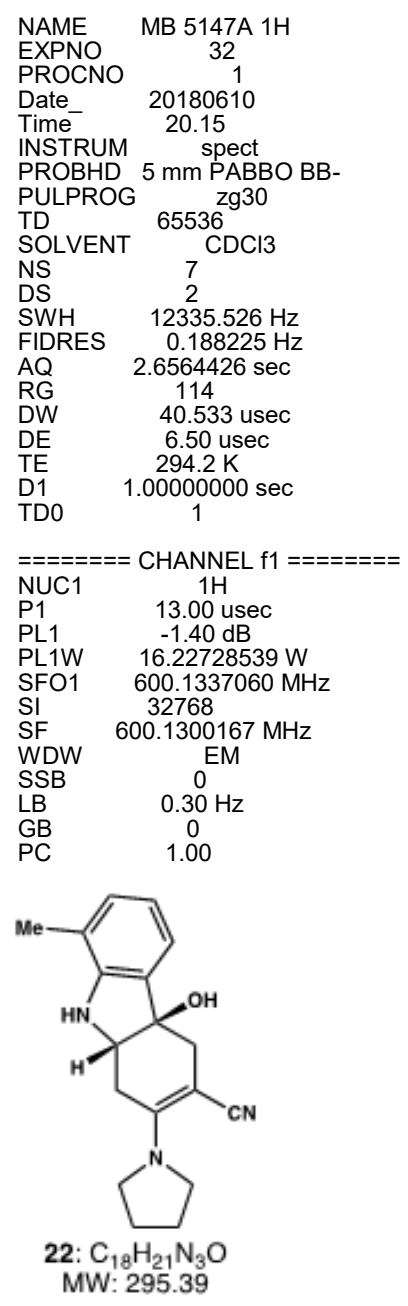

PROBHD 5 mm PABBO BB-

PULPROG $\mathrm{zg} 30$

SWH

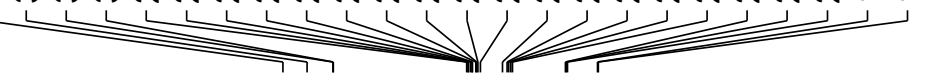

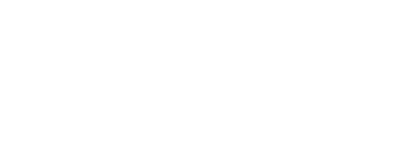

14
13
12

11

11

10

9

9

8

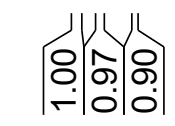
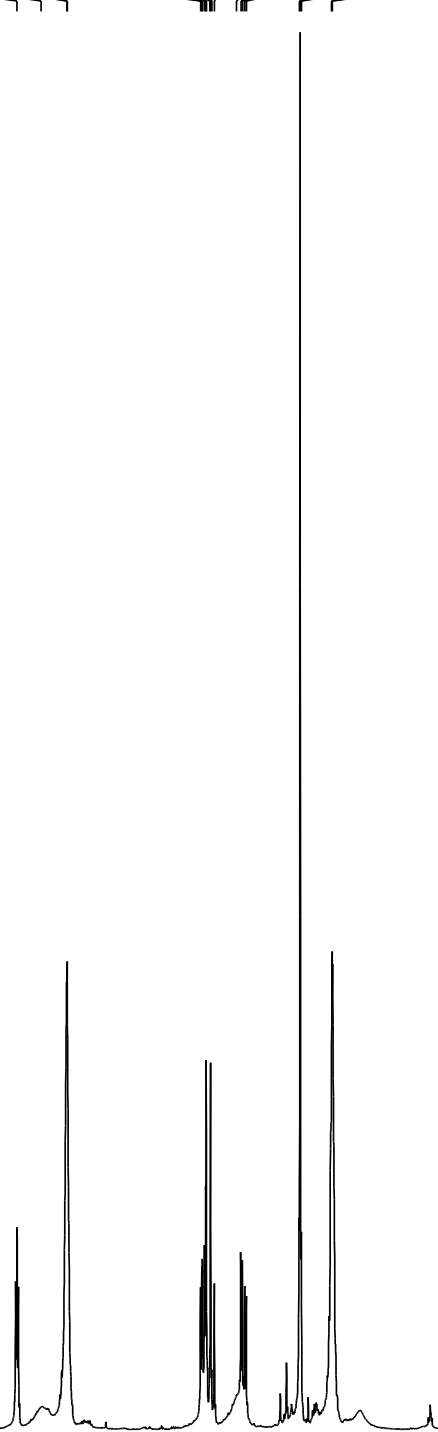


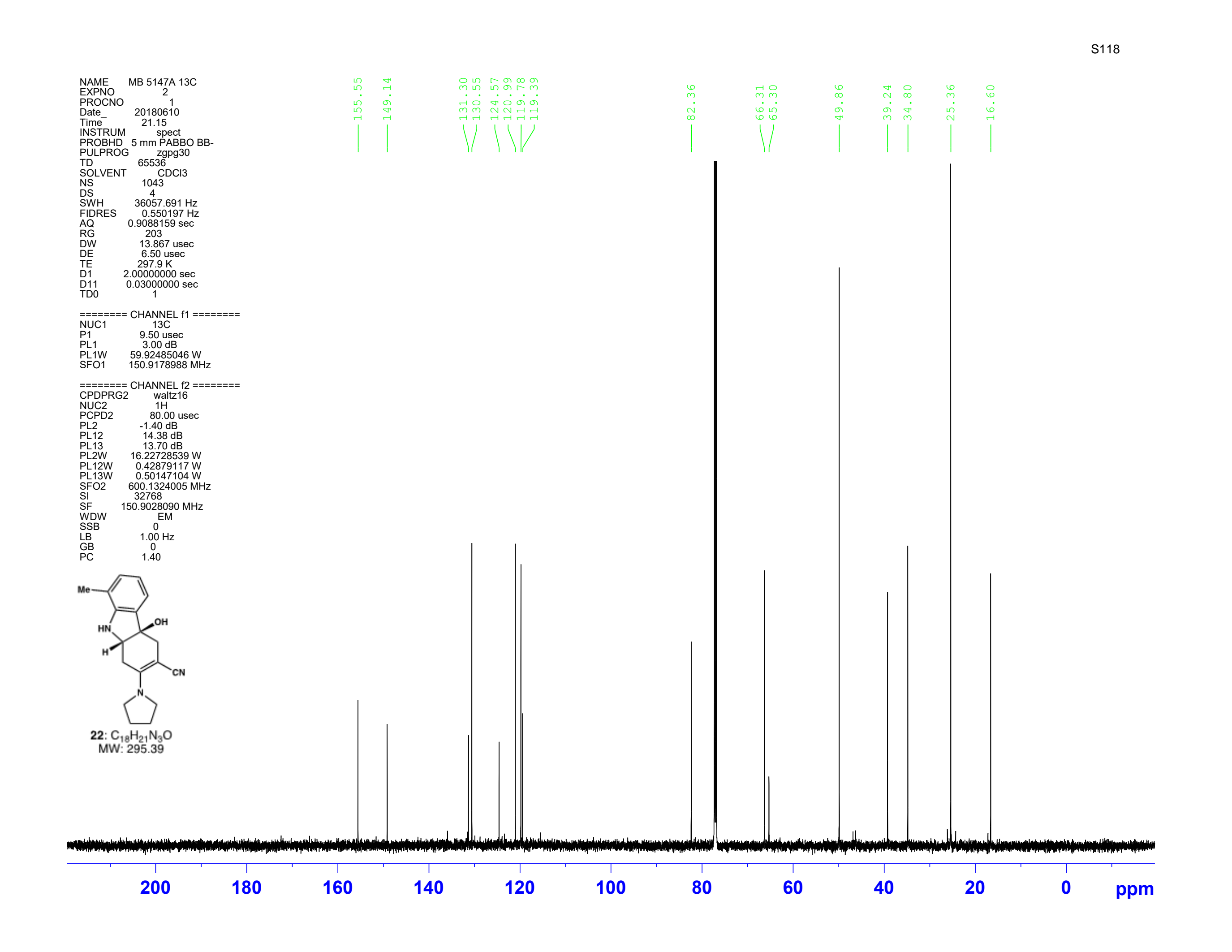




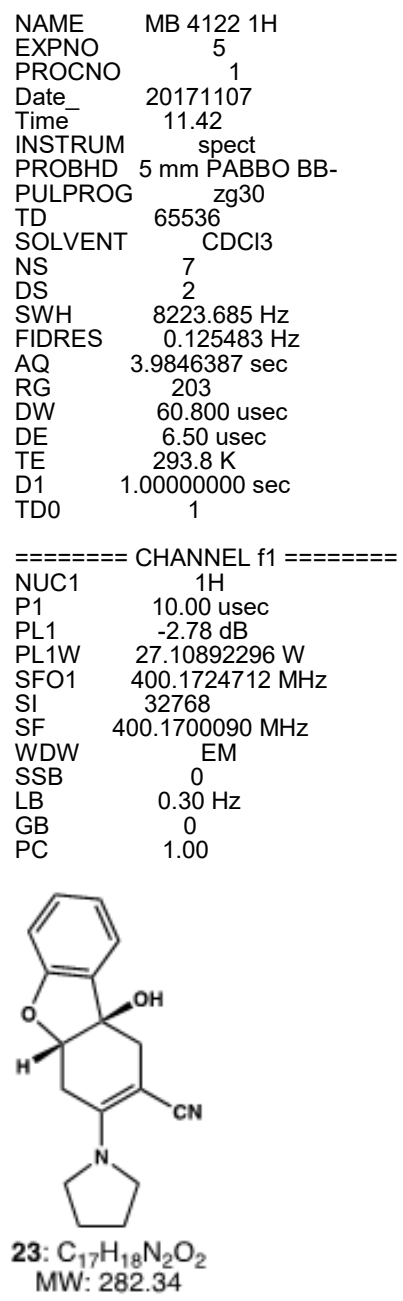

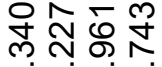
Ni⿺辶寸

YII

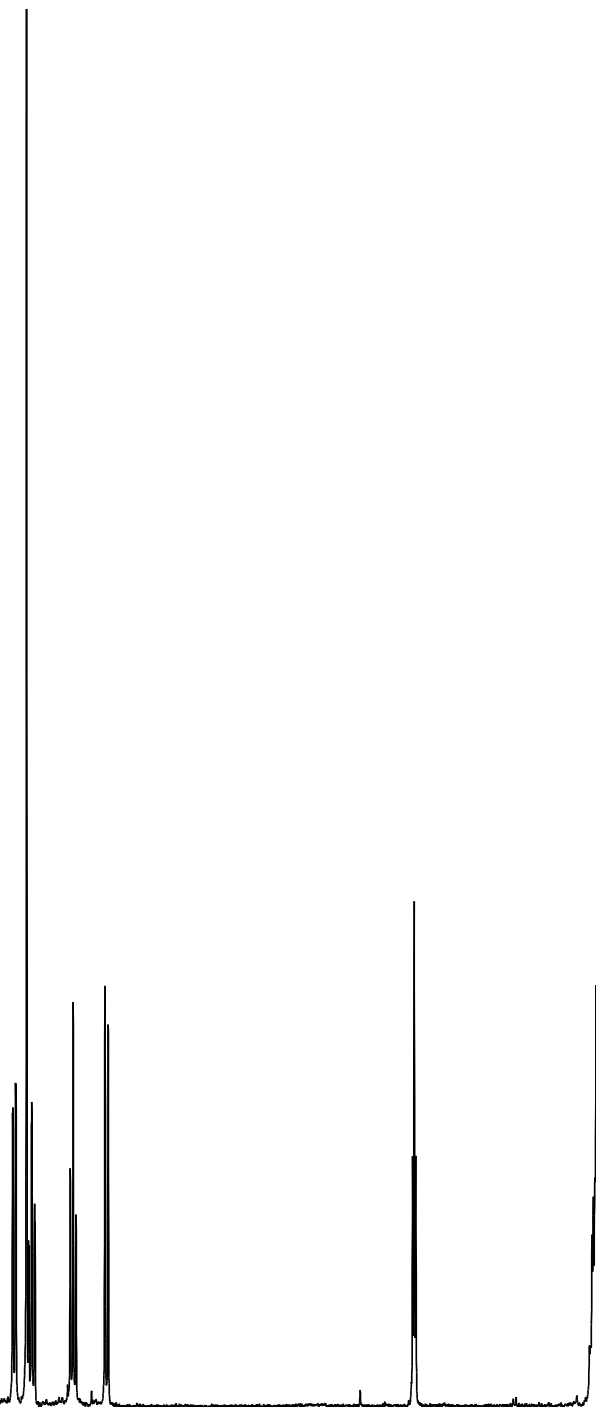

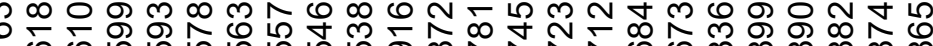

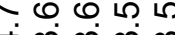
m m m m m m

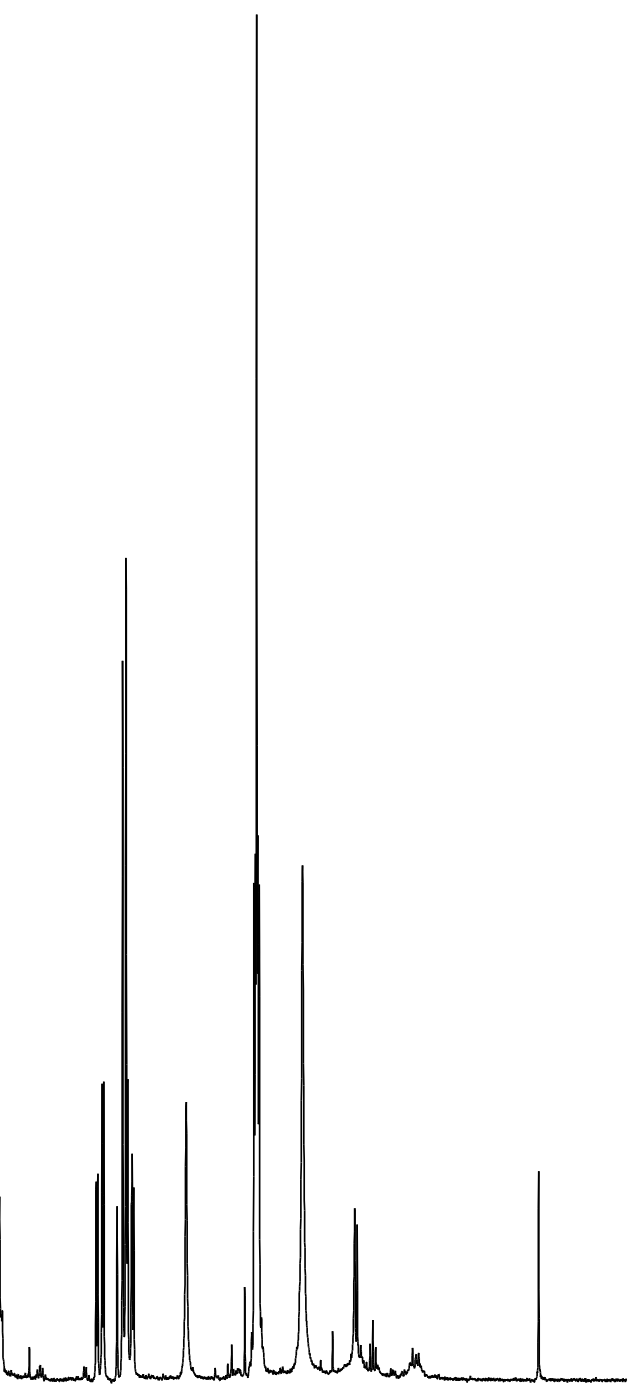

14 13 12 11 10 9

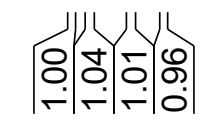
6 5 |ู่) 4

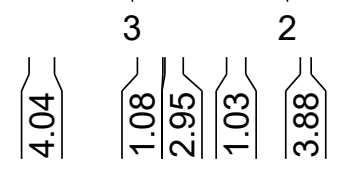





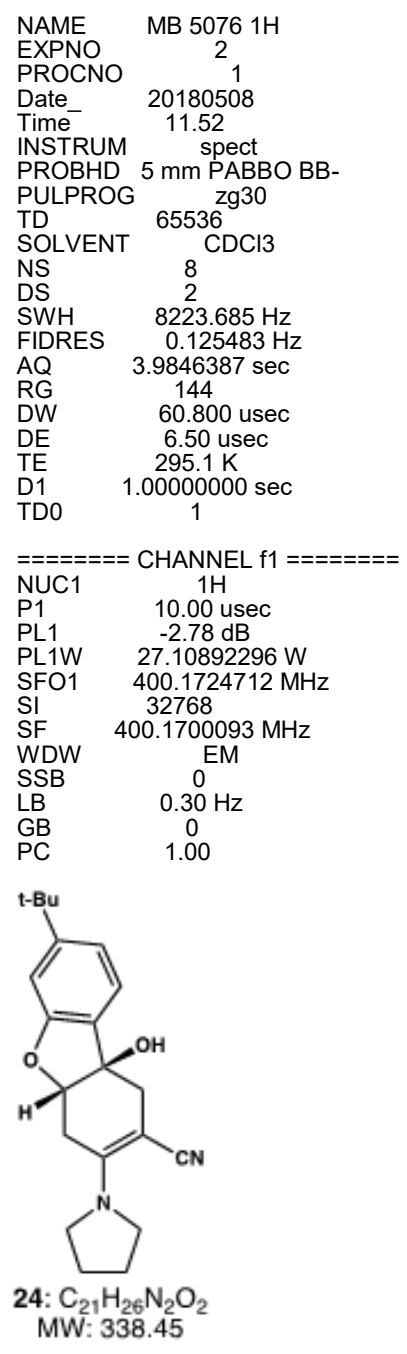

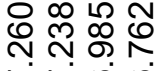

N

V11

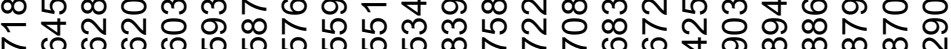
ウ
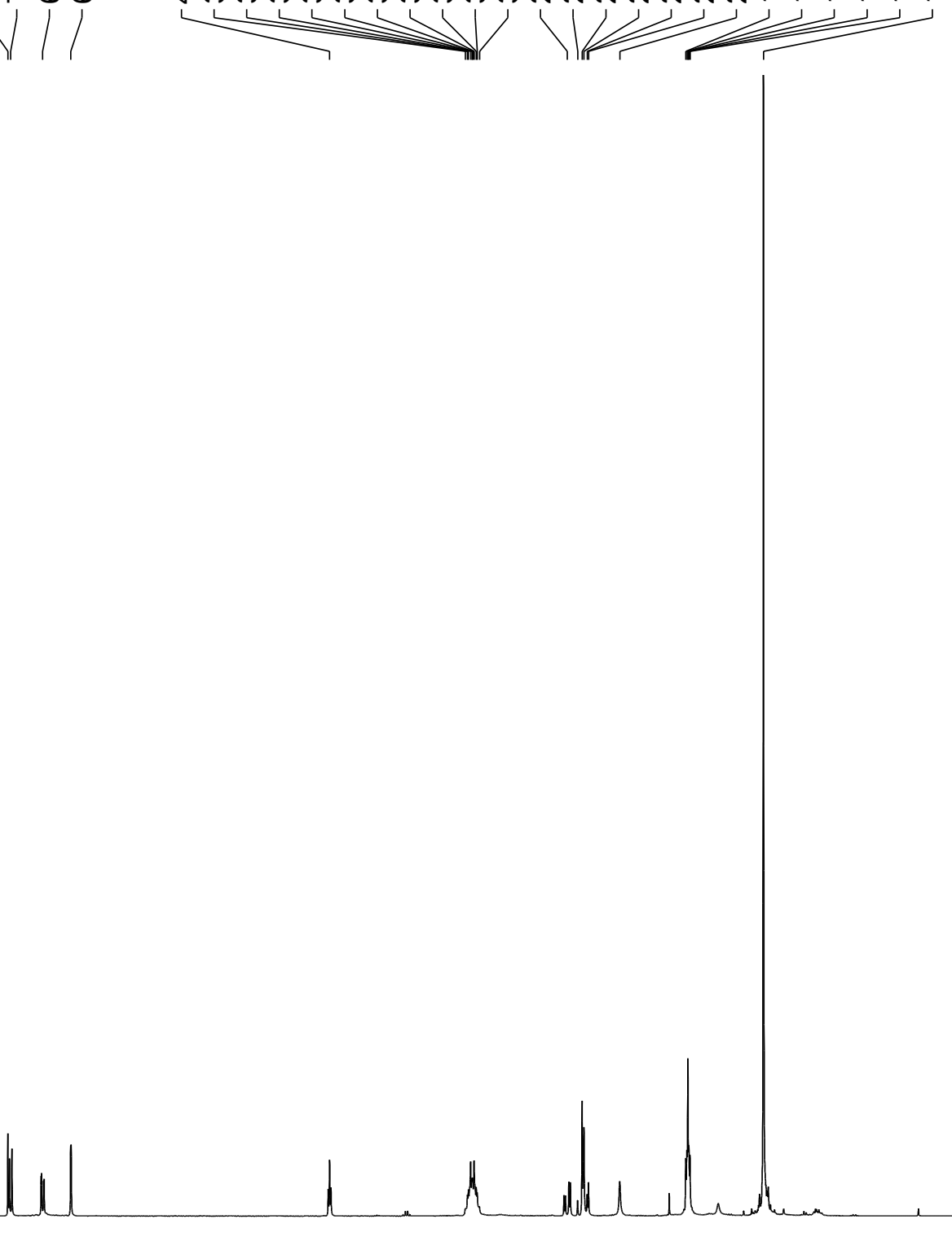

14

13
12

11

10

9

9

8

8

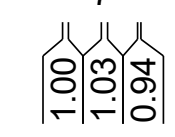

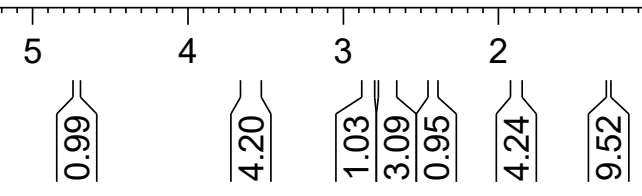




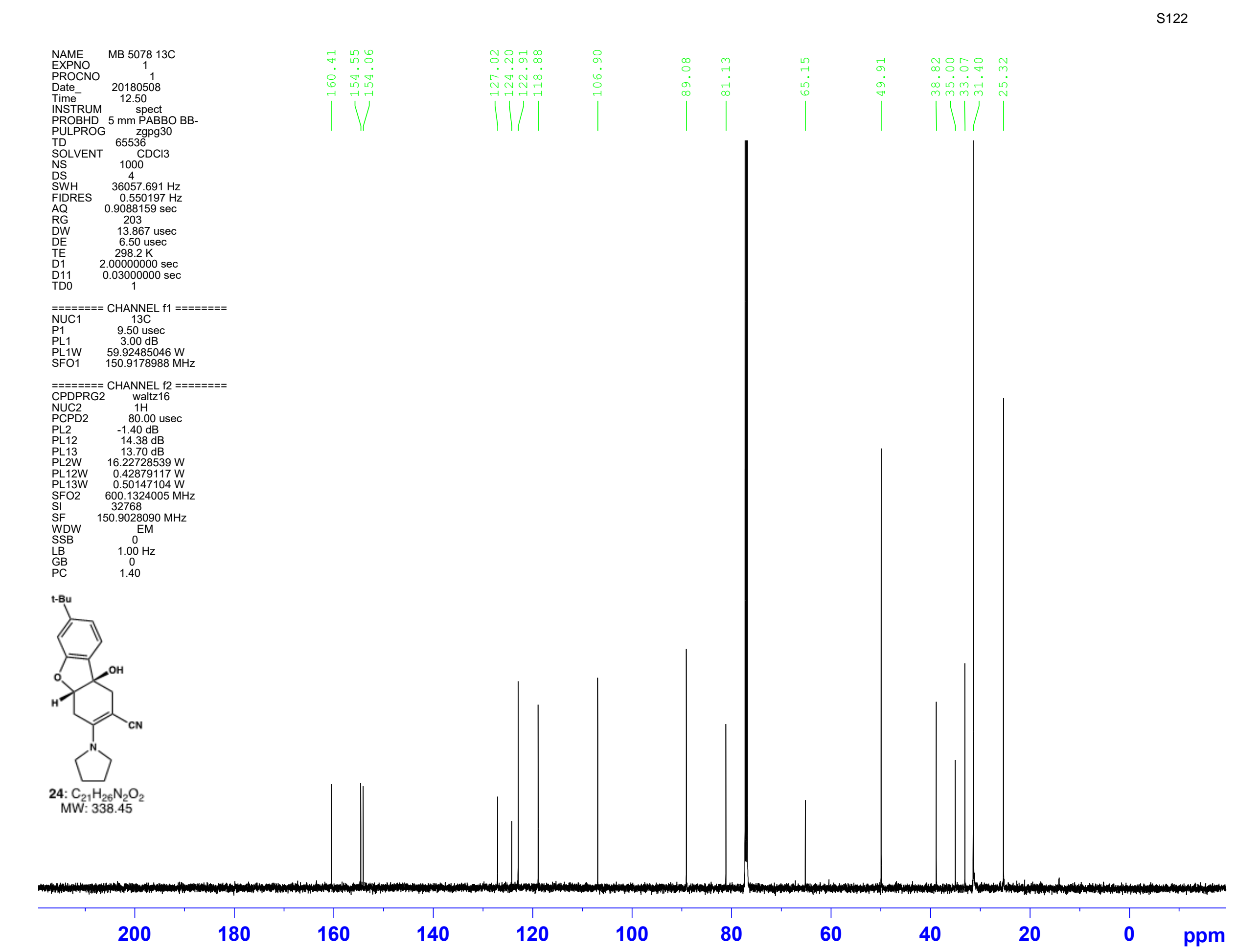




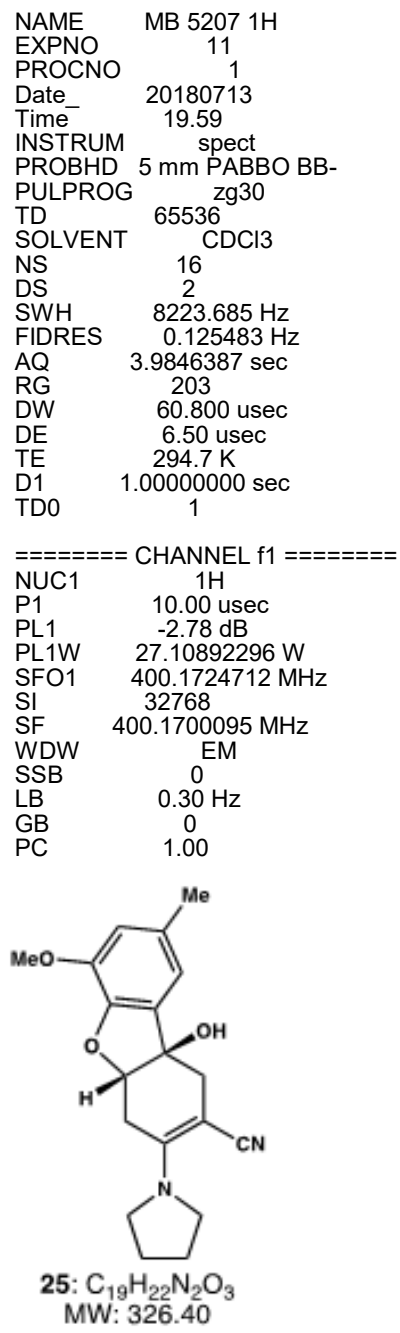

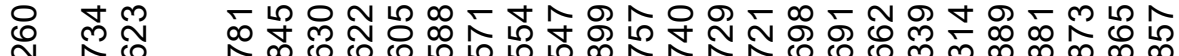
শ

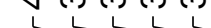

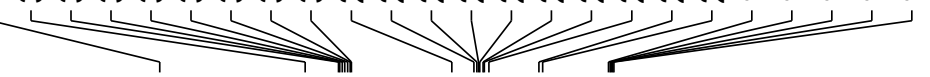

(2)

DW 60.800 usec

6.50 usec

$========$ CHANNEL $\mathrm{f} 1$ ========

SFO1 $\quad 400.1724712 \mathrm{MHz}$

SF $\quad 400.1700095 \mathrm{MHz}$

WDW $\quad$ EM

LB $\quad 0.30$
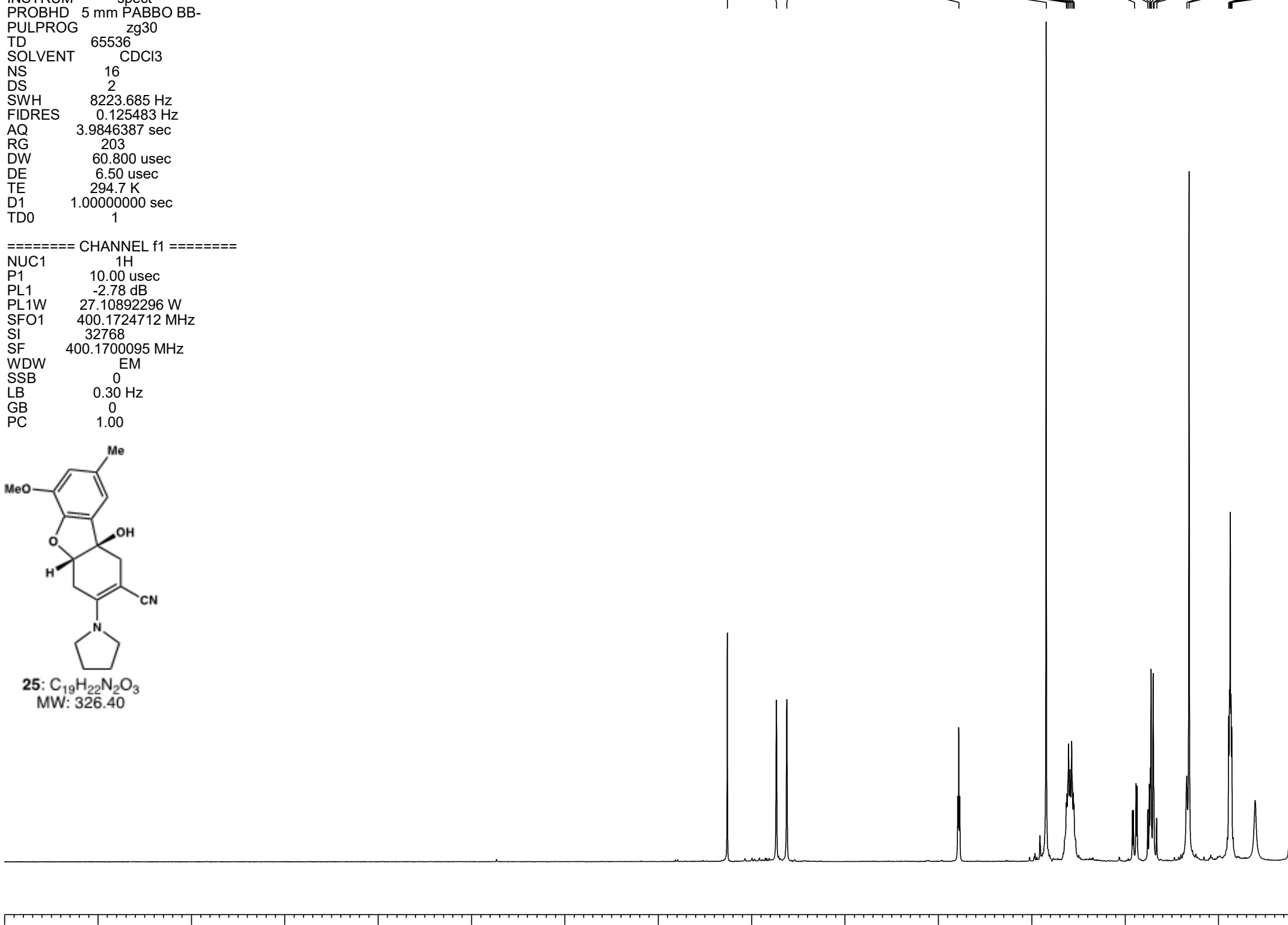

14 


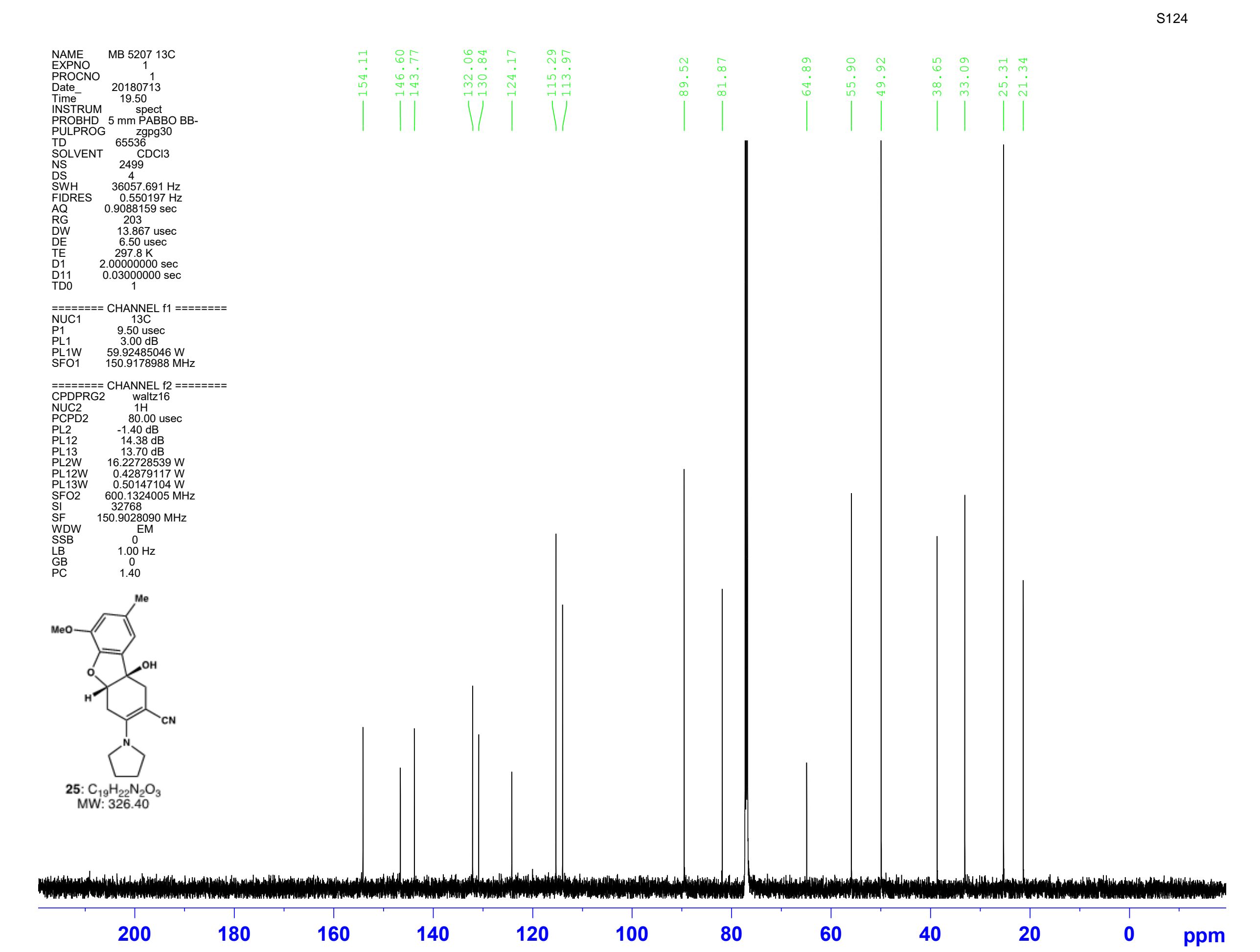




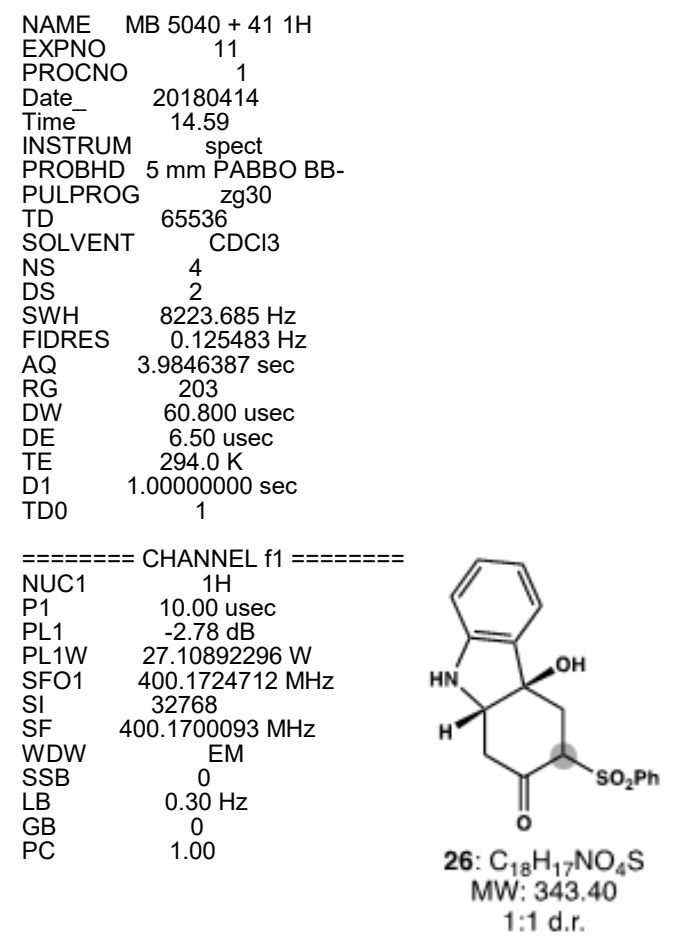

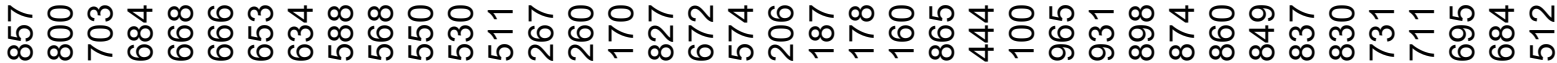
$\infty$ ب $\ldots$

5 (1)

PULPROG $2 \mathrm{zg} 30$

SWH

TD0 1.0001000
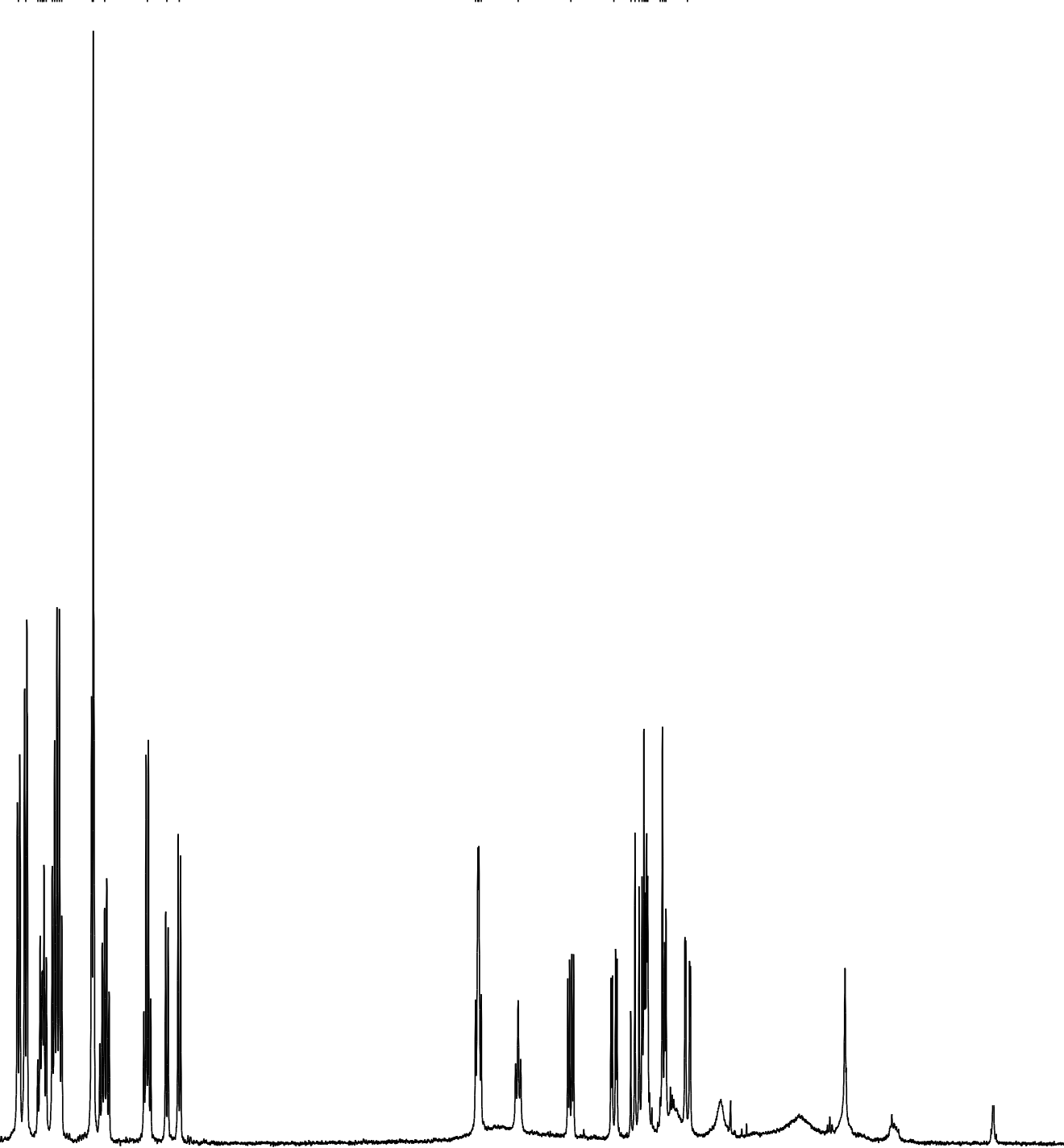

14

13

12

11

10

9

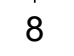

7

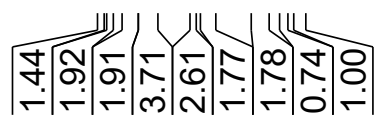

5

43

2

它 


$$
w_{3}
$$




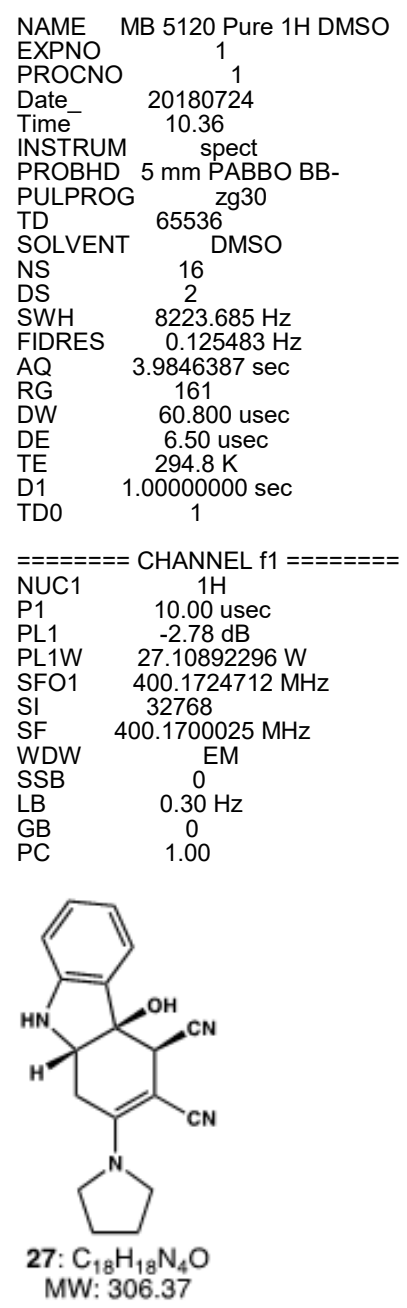

을 רי

11111

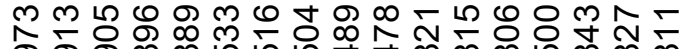
ம்

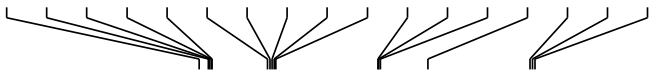

MW: 306.37 

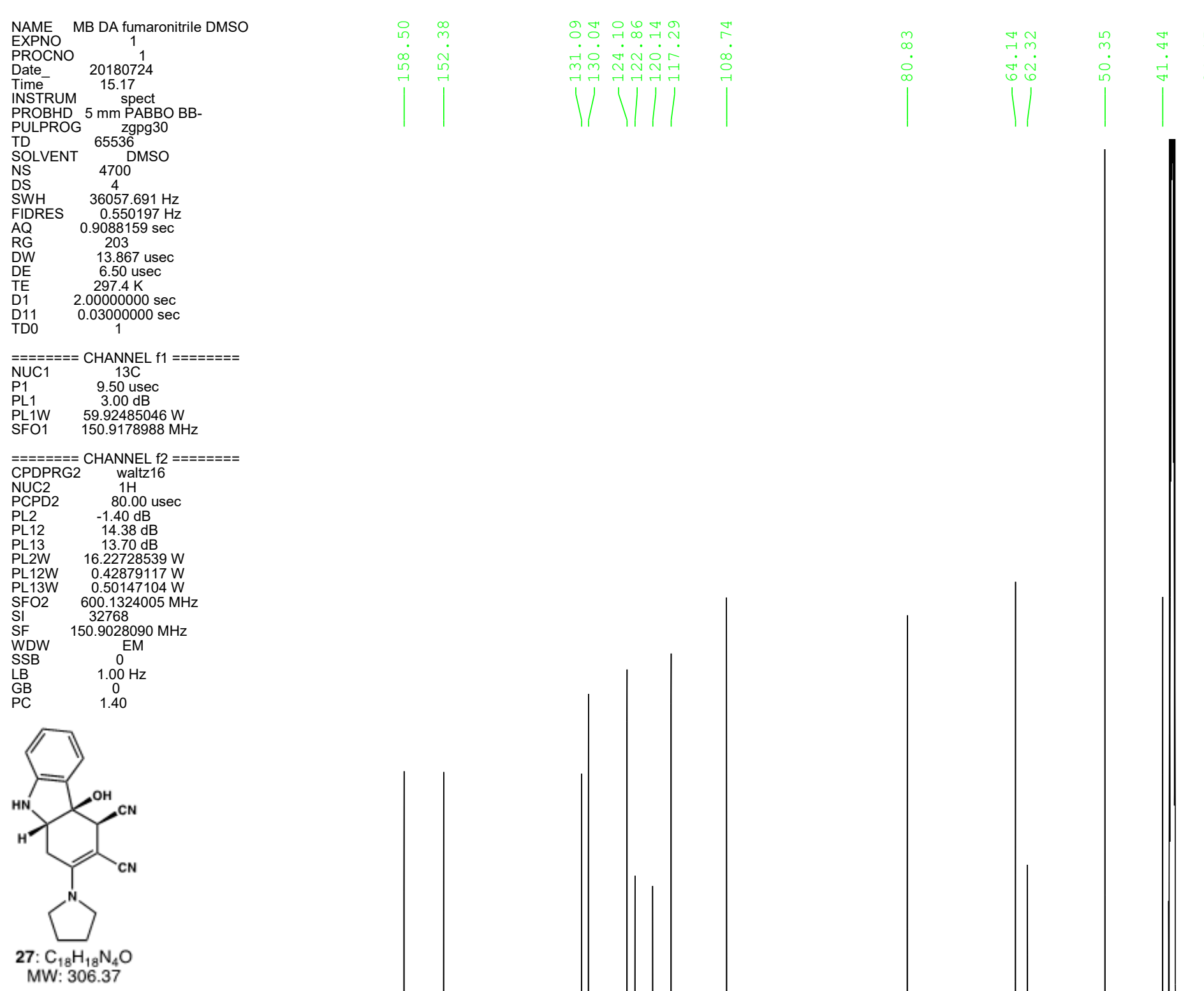


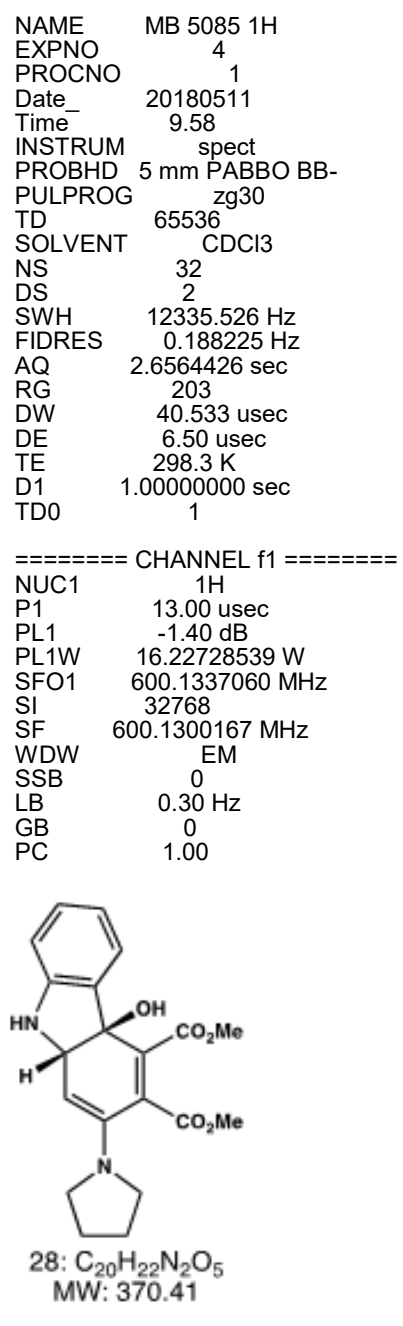

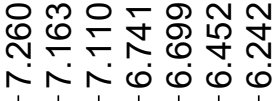

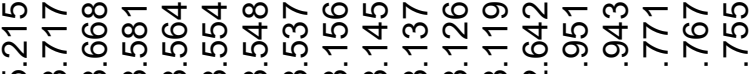

रा।

这

INSTRUM spe

PABBO BB-

SOLVENT CDC

$=======$ CHANNEL $f 1$ =======

SFO1 $\quad 600.137060 \mathrm{MHz}$

SF $\quad 600.1300167 \mathrm{MHz}$

WSB $\quad 0 M$

$\mathrm{PC}$

$30 \mathrm{~Hz}$
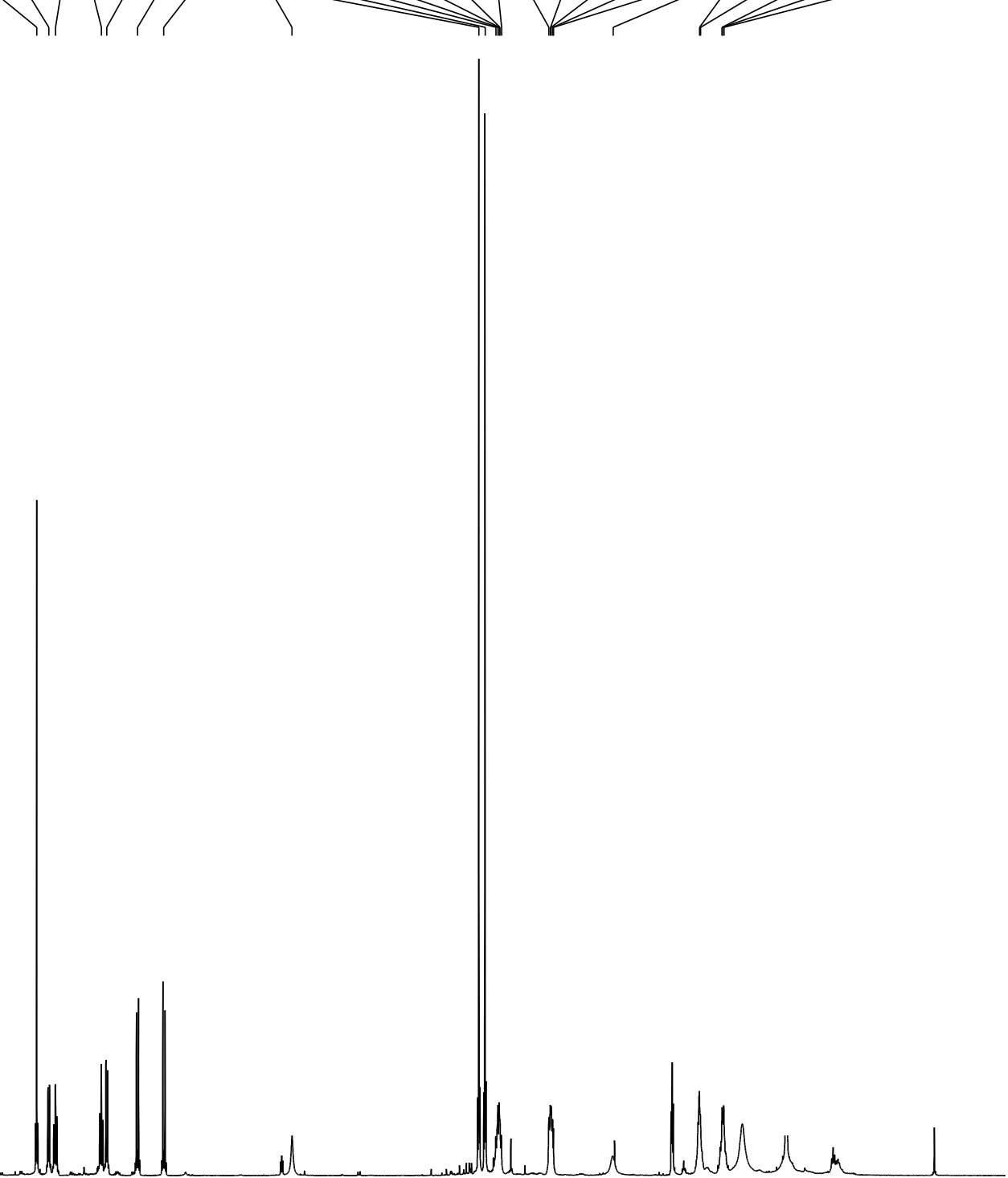

$1+1+1+11$

5




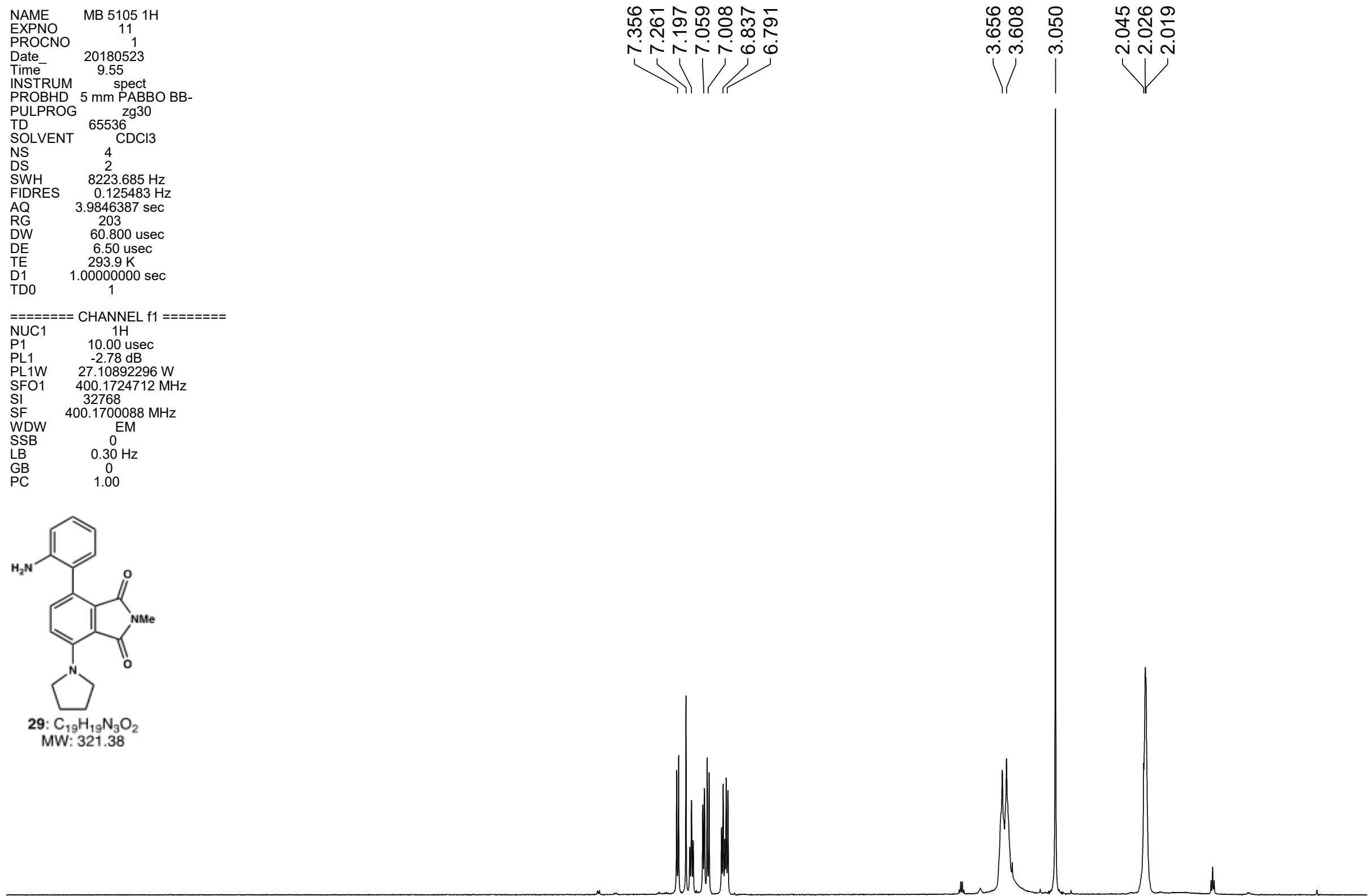

14

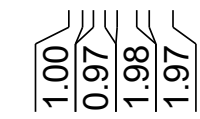

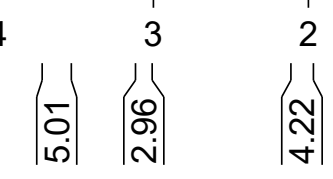




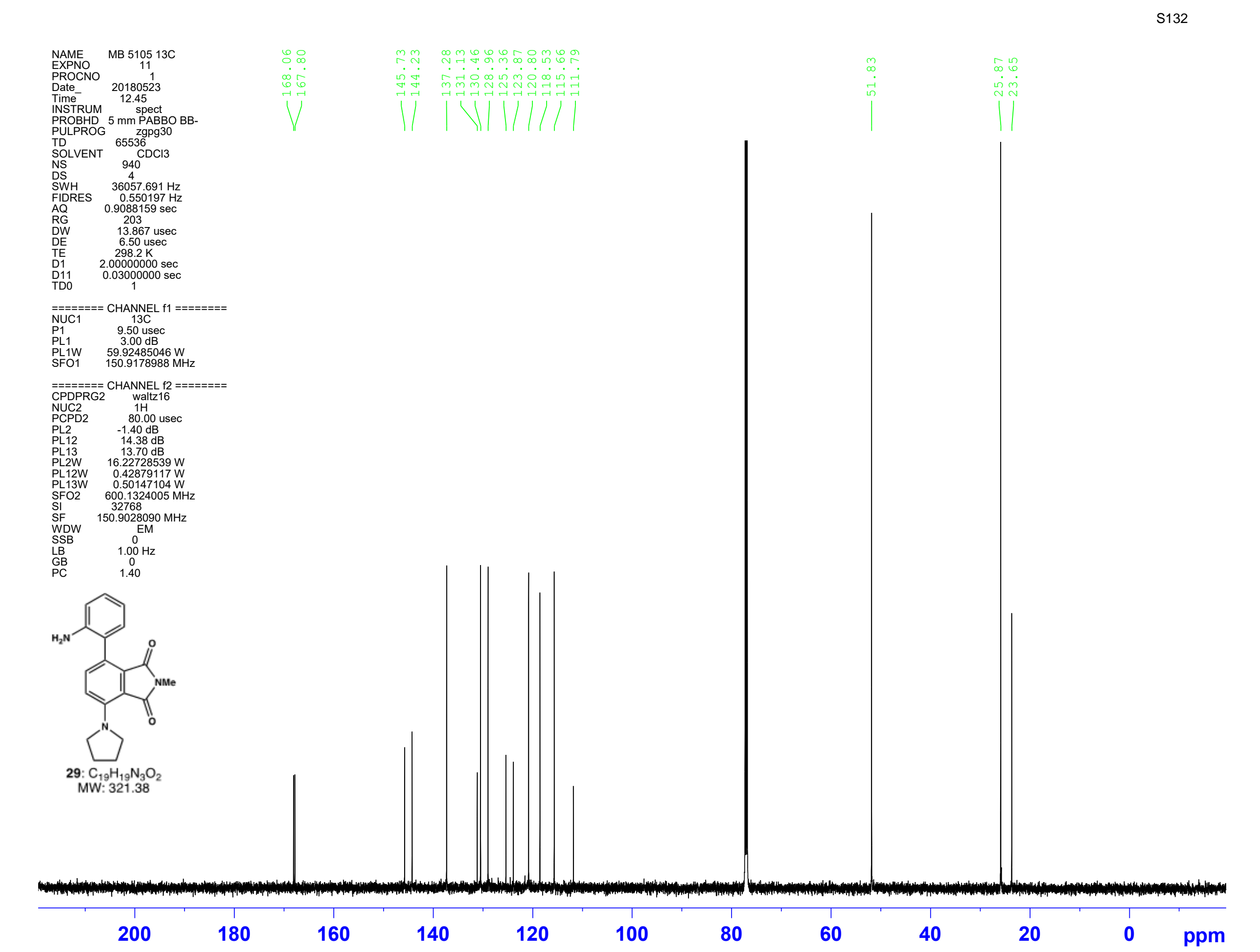




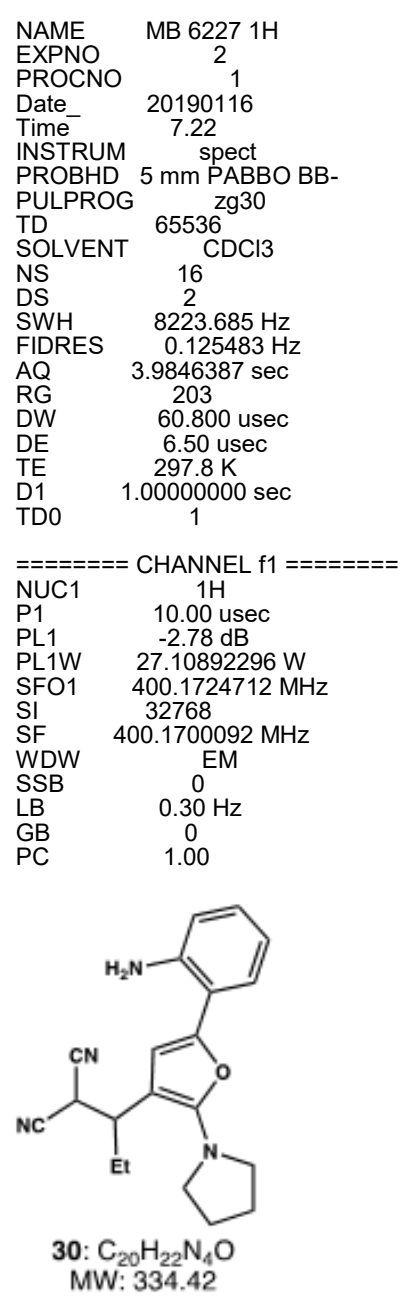

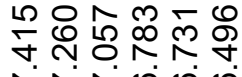

co

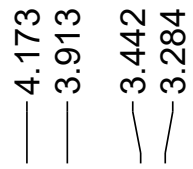

\ij|

ABBO BB-

11111

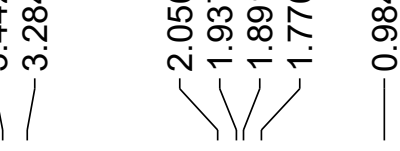

$=======$ CHANNEL $\mathrm{f} 1=======$

SFO1 $4001724712 \mathrm{MHz}$

SF $\quad 400.1700092 \mathrm{MHz}$

SSB $\quad 0$

GB $\quad 0.30$

MW: 334.42

$14 \quad 13$

12

11

9

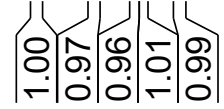

5

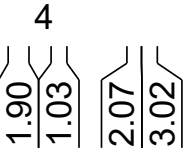

3

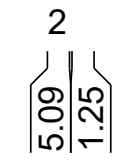

$\left|\begin{array}{l}1 \\ \frac{8}{m}\end{array}\right|$ 


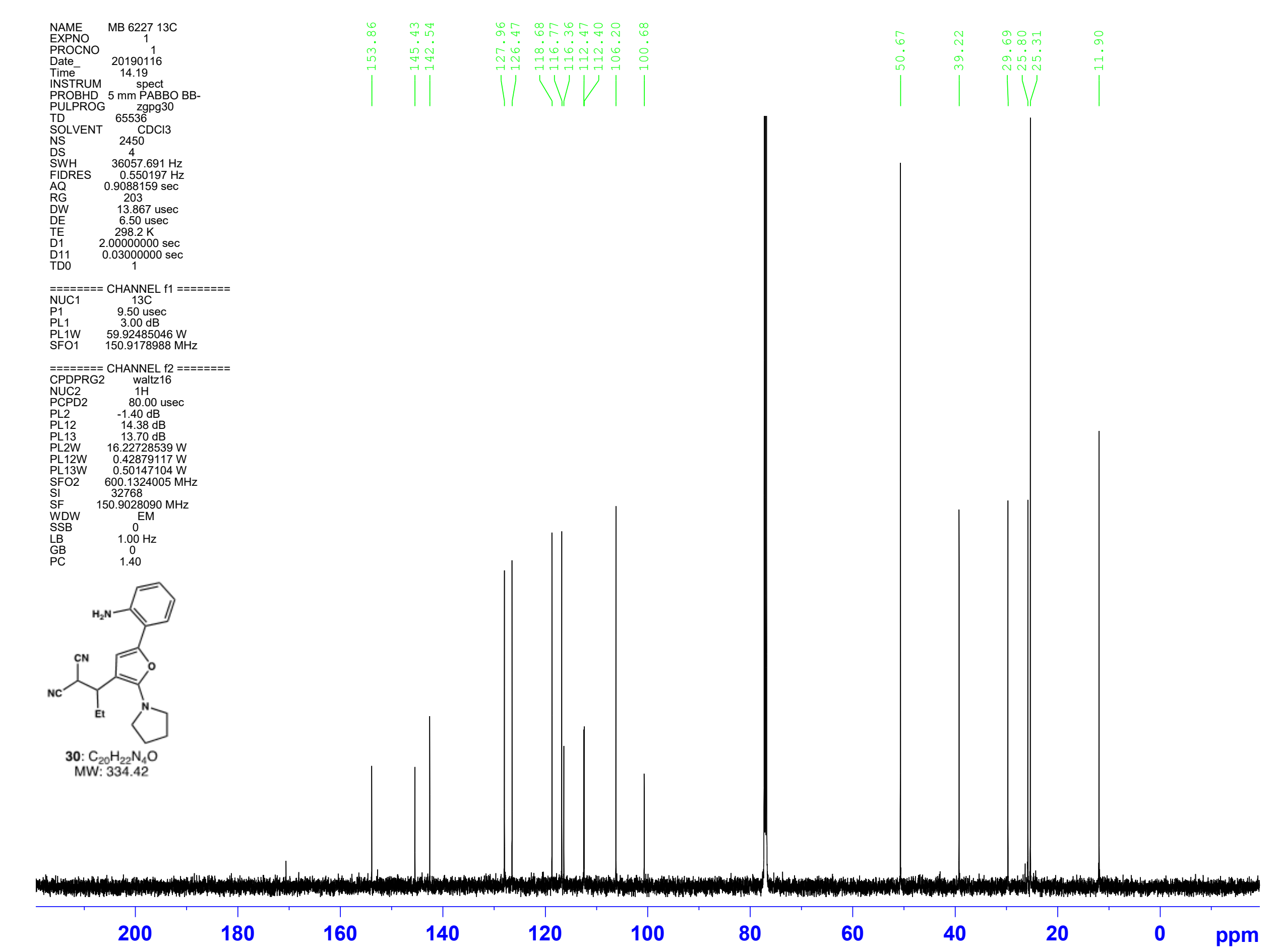



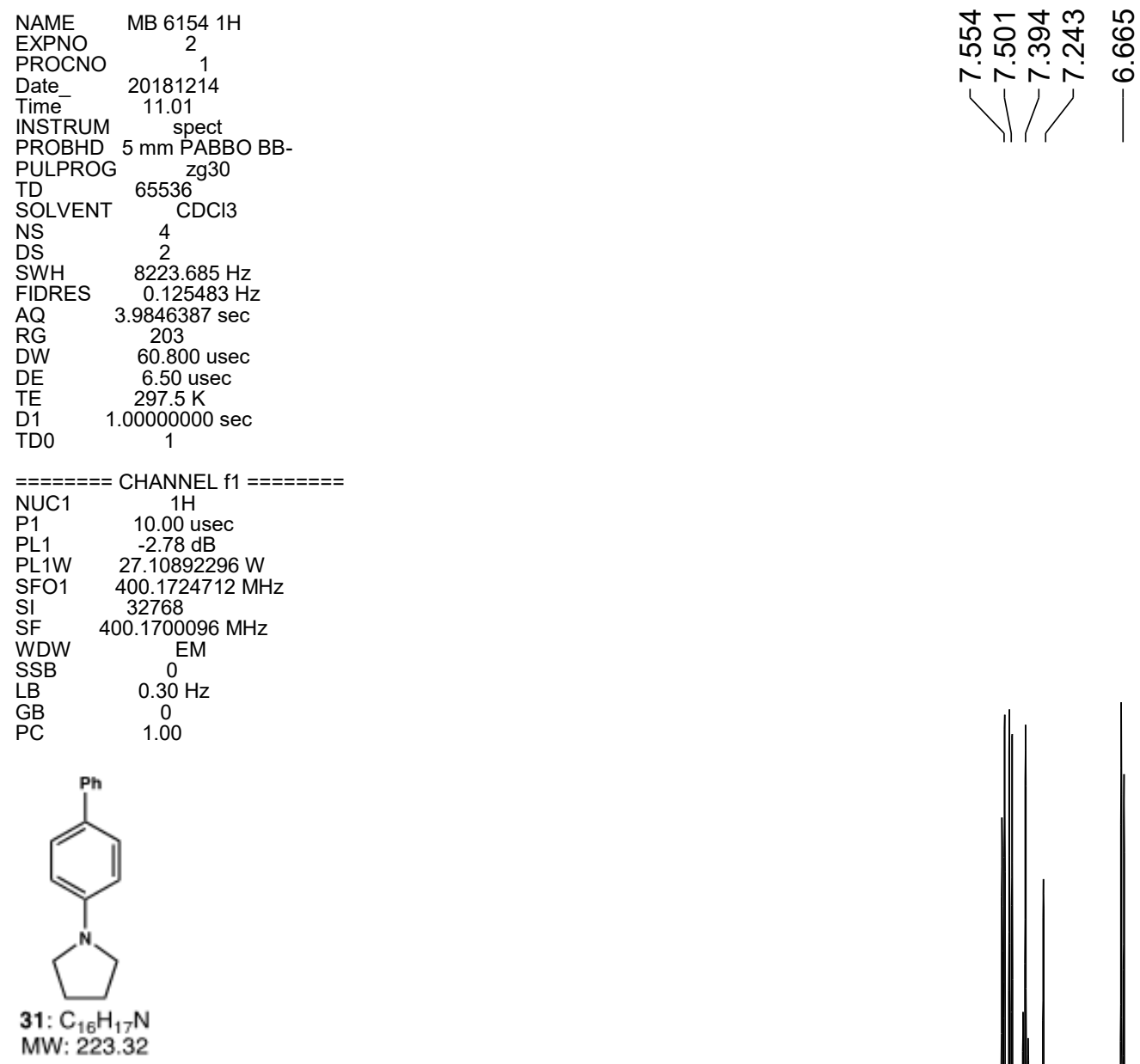

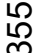

ळ.

PROBHD $5 \mathrm{~mm}$ PABBO BB-

PULPROG $\quad \mathrm{zg} 30$

SOLVENT $\quad \mathrm{CDCl} 3$

DS

SWH $\quad 8223.685 \mathrm{~Hz}$

$30.125873 \mathrm{~Hz}$

203

DE $\quad 6.50$ us

D1 1.00000000

$========$ CHANNEL $\mathrm{f} 1$ ========

27.10892296

$\begin{array}{ll}\text { PL1W } & 27.10892296 \mathrm{~W} \\ \text { SFO1 } & 400.1724712 \mathrm{MHz}\end{array}$

$\begin{array}{ll}\text { SI } & 32768 \\ \text { SF } & 400.1700096 \mathrm{MHz}\end{array}$

WSB

LB

${ }^{\mathrm{E}}$
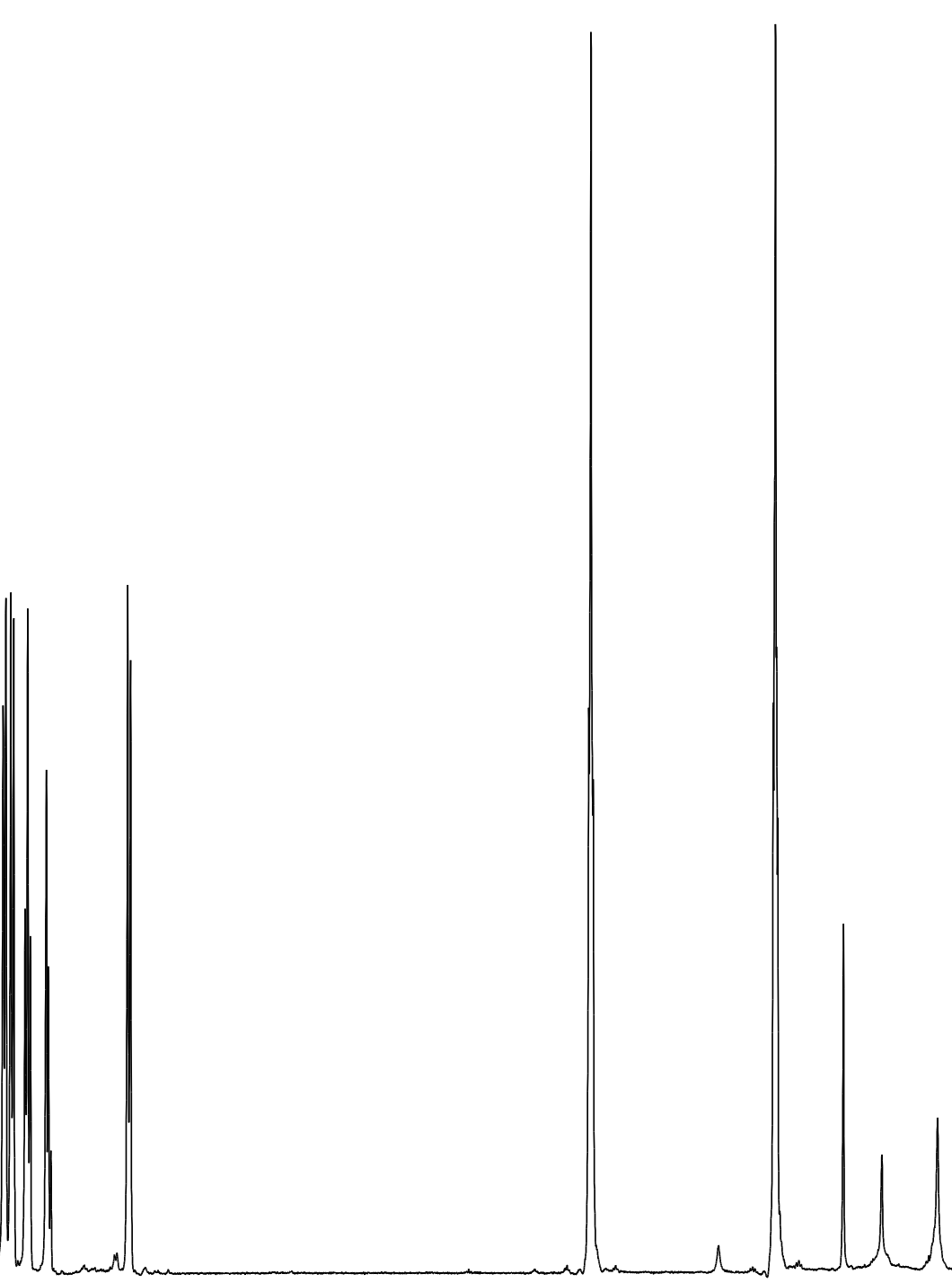

$14 \quad 13$

12

11

10

9

8

7

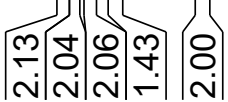

$\left|\begin{array}{c}4 \\ 0 \\ \dot{j}\end{array}\right|$

2

$\left|\begin{array}{l}0 \\ 0 \\ j\end{array}\right|$ 